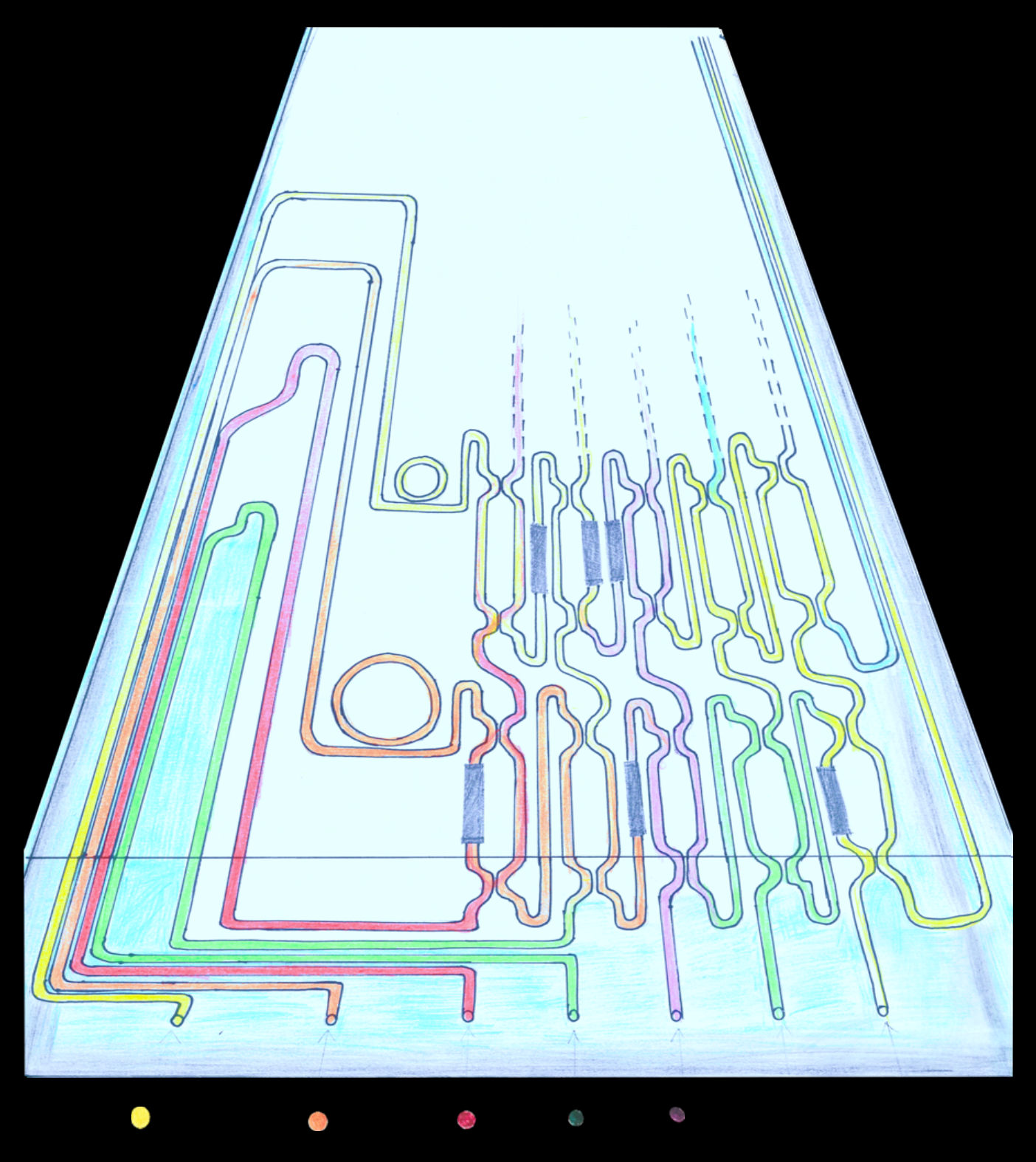




\section{INTEGRATED PROGRAMMABLE WAVEGUIDE CIRCUITS FOR CLASSICAL AND QUANTUM PHOTONIC PROCESSING}

by

Caterina Taballione 



\title{
INTEGRATED PROGRAMMABLE WAVEGUIDE CIRCUITS FOR CLASSICAL AND QUANTUM PHOTONIC PROCESSING
}

\author{
DISSERTATION
}

to obtain

the degree of doctor at the University of Twente, on the authority of the rector magnificus, prof. dr. T.T.M. Palstra,

on account of the decision of the Doctorate Board, to be publicly defended

on the $11^{\text {th }}$ of July 2019 at 14:45 hours

by

\section{Caterina Taballione}

born on the $19^{\text {th }}$ of July 1988

in Rome, Italy 
This dissertation has been approved by:

Supervisor(s):

Prof. Dr. Klaus-J. Boller

Prof. Dr. Pepijn W. H. Pinkse

Cover design: Hand drawing by Caterina Taballione

Printed by: Gildeprint, Enschede, The Netherlands

ISBN: $\quad$ 978-90-365-4803-8

DOI: $\quad$ https://doi.org/10.3990/1.9789036548038

(C) 2019 Enschede, The Netherlands. All rights reserved. No parts of this thesis may be reproduced, stored in a retrieval system or transmitted in any form or by any means without permission of the author. Alle rechten voorbehouden. Niets uit deze uitgave mag worden vermenigvuldigd, in enige vorm of op enige wijze, zonder voorafgaande schriftelijke toestemming van de auteur. 


\section{Graduation Committee}

Chairman/secretary

Supervisor(s)

Committee Members:
Prof. Dr. J. L. Herek, University of Twente

Prof. Dr. K.-J. Boller, University of Twente

Prof. Dr. P. W. H. Pinkse, University of Twente

Prof. Dr. S. Barz, Universität Stuttgart

Dr. A. L. Tchebotareva, TNO

Dr. Ir. S. Faez, Utrecht University

Prof. Dr. Ir. W. G. van der Wiel, University of Twente

Prof. Dr. J. Schmitz, University of Twente

\section{$\widehat{N W O} \mid \begin{aligned} & \text { Applied and } \\ & \text { Engineering Sciences }\end{aligned}$}

The work presented in this thesis was carried out at the Laser Physics and Nonlinear Optics group, Department of Science and Technology, Mesa+ Institute for Nanotechnology, University of Twente, P.O. Box 217, 7500 AE Enschede, The Netherlands. This work is part of the research program Memphis II with project number 13532, which is (partly) financed by the Netherlands Organization for Scientific Research (NWO). 



\section{Contents}

1. Introduction ................................................................................................ 11

2. Theoretical background.............................................................................. 19

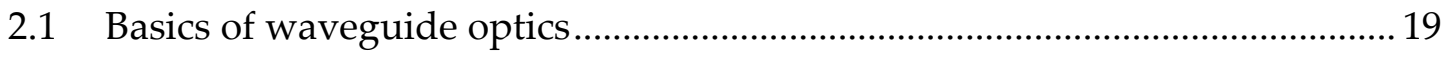

2.1.1 Thermal tuning ........................................................................................ 22

2.1.2 Optical modes for channel waveguides ..................................................... 23

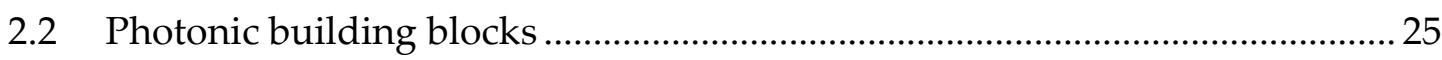

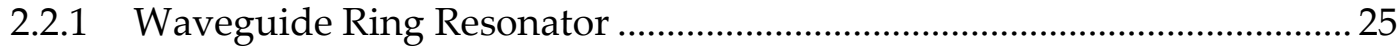

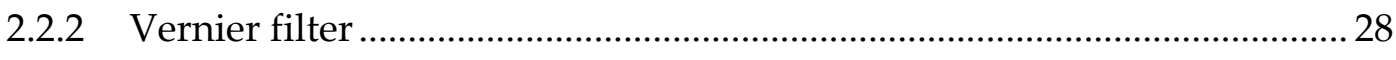

2.2.3 Mach-Zehnder interferometer ................................................................ 29

2.2.4 An imperfect Mach-Zehnder interferometer................................................ 31

2.2.5 Network of Mach-Zehnder interferometers.................................................. 33

2.3 From classical to quantum linear optical circuits.................................................. 35

2.3.1 Quantum linear optical circuits..................................................................... 36

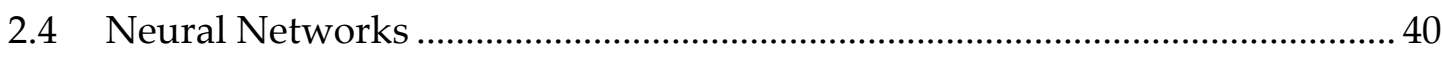

3. Smart wavelength meter based on ring resonators................................................ 45

3.1 Towards a temperature-drift-immune wavelength meter based on a single

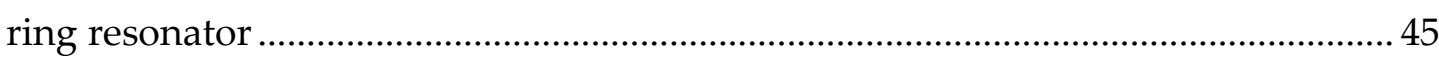

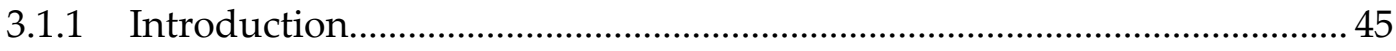

3.1.2 Principle of data readout algorithm ......................................................... 46

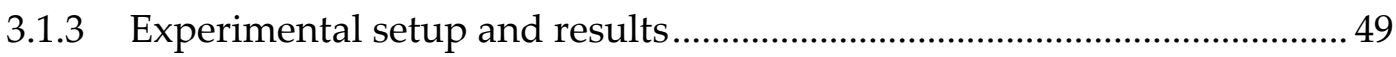

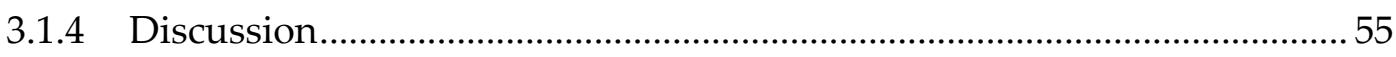

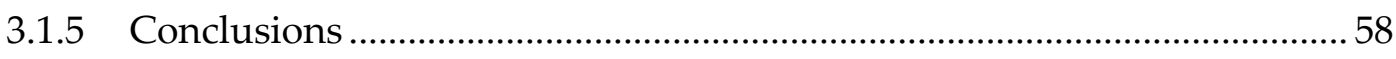

3.2 Range-extended wavelength meter based on double ring resonators in Vernier

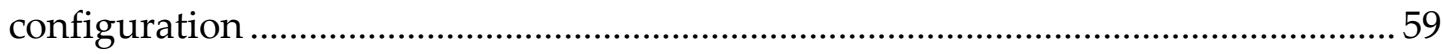

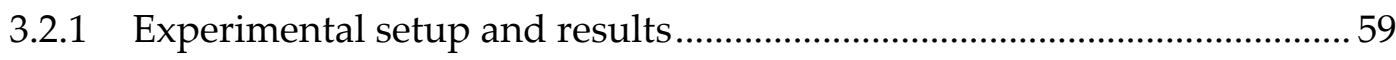

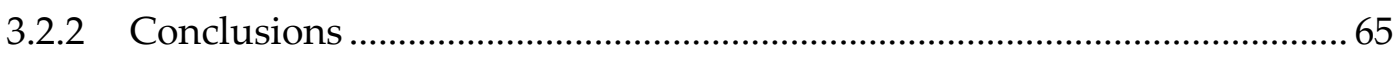

4. A reconfigurable linear optical circuit.............................................................69

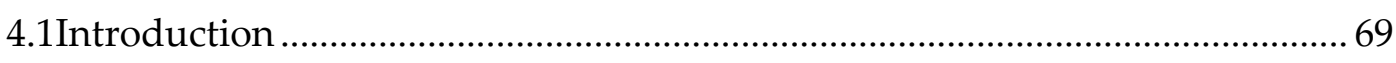

4.2 Multi-stage architecture and experimental setup ………………………......... 70 


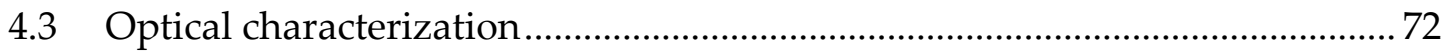

4.3.1 Characterization of the tunable beam splitters ...................................... 73

4.3.2 Characterization of the phase shifters .................................................... 76

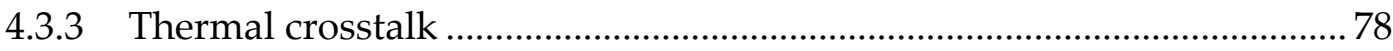

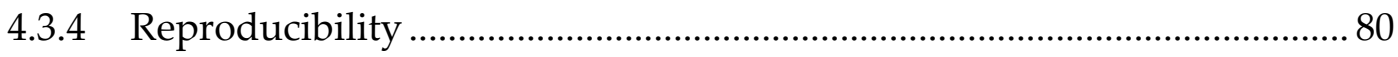

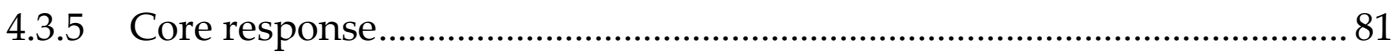

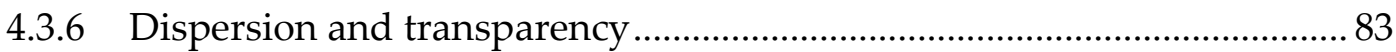

4.4 Improving the response of an imperfect linear optical circuit......................... 85

4.4.1 Implementing arbitrary unitary transformations on imperfect linear

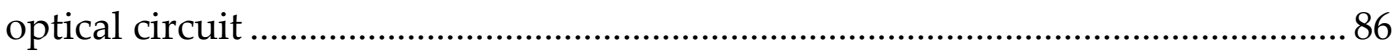

4.4.2 Overcoming fabrication imperfections with numerical optimization..... 91

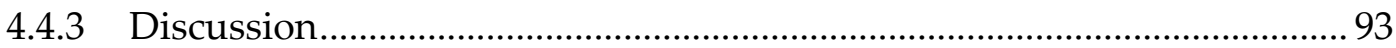

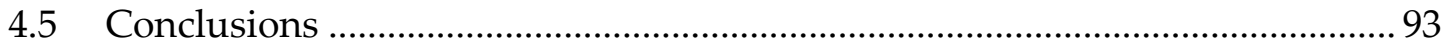

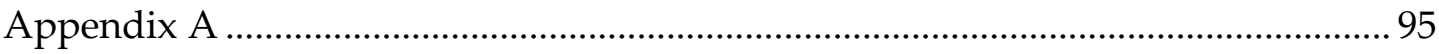

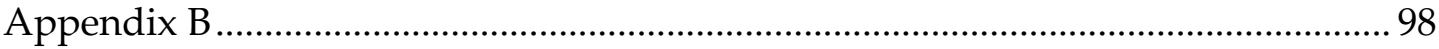

5. Quantum photonic processing on a reconfigurable linear optical circuit 101

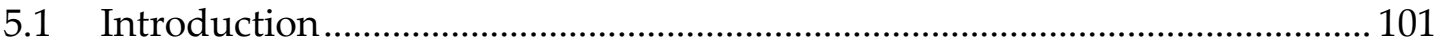

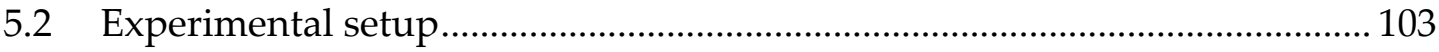

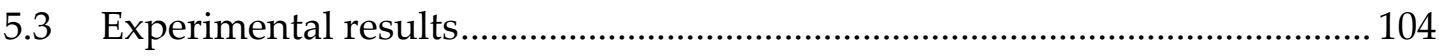

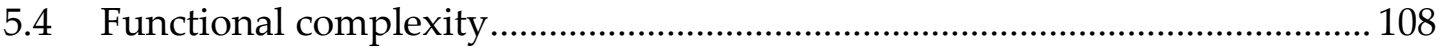

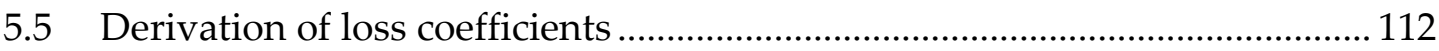

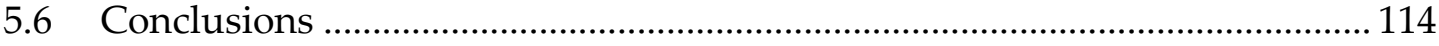

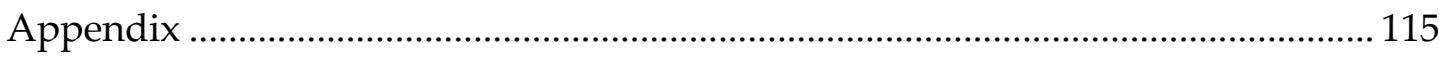

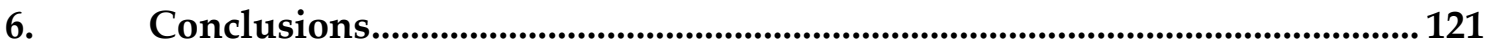

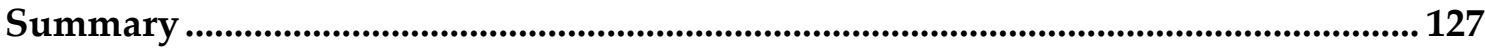

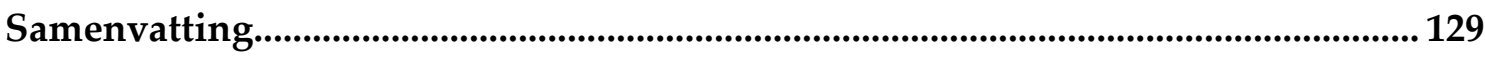



A Giorgio e alla mia famiglia. 


\section{Introduction}

Integrated photonics [1] is a developing field that increasingly incorporates various optical functionalities with waveguide technology on a chip. As a key enabling technology, the field promises to have a large impact on many potential and emerging applications, such as medical diagnostics [2], water quality and pollution monitoring $[3,4]$, chemical sensing [5], data communication [6], microwave photonic processing [7], optical metrology and sensing [8], and photonic information processing exploiting quantum effects [9-13].

In all these applications, the main strength obtained by integrating photonic circuits on a chip is the inherent phase stability imposed by integration, which easily provides better than subwavelength long-term stability. The interferometric stability and reliability of integrated photonics is, usually, much higher than using either bulk photonic approaches or fiber optics. In addition, state-of-the-art integrated photonics offers ways to exert external electronic control of the optical phases of the light that propagates through integrated waveguides, and significant technological maturity of phase control implementation. This combination of inherent stability and external controllability enables reliability in connection with complexity.

Providing complexity and control is the key towards high functionality of optical circuits. This thesis aims on using photonics integration in combination with electronic control of optical phases to demonstrate progress towards functionalities for wavelength metrology and linear optical quantum processing.

Two types of material platforms can be recognized in integrated photonics. The first is the semiconductor waveguide platform which is ideal to generate, detect and modulate light on chips or to employ nonlinear optical effects. For example, semiconductor indium phosphide (InP) lasers emitting around $1.55 \mu \mathrm{m}$ wavelength are very important in applications such as optical communication. On one hand, the relatively small bandgap of semiconductors enables efficient optical processes such as, e.g., stimulated emission for generating light. On the other hand, due to the same reason, the propagation of light in this platform imposes strong loss and short absorption and scattering lengths. Such propagation loss translates into limiting the extent to which interferometric approaches and devices, e.g., using semiconductor resonators and Mach-Zehnder interferometers, can be observed and exploited.

The second type of platform is based mainly on dielectric materials, where due to a relatively large electronic bandgap the propagation loss is significantly reduced 


\section{Introduction}

and nonlinear effects can usually be neglected. Dielectric waveguide platforms with low propagation loss are ideal for optimally exploiting one of the most important properties of light on chips, namely its coherence, through optical superposition and interference. In fact, optical cavities and interferometers such as ring resonators and Mach-Zehnder interferometers (MZIs) have become two of the most exploited optical structures in dielectric waveguide platforms as, e.g., spectral filters such as wavelength-division multiplexers, optical sensors, or optical delay lines. The low-loss advantage of integrated photonics based on dielectric materials is most apparent when aiming on applications that make use of multi-beam interference for high performance at chip-size dimensions. In addition, the linear optical light propagation in dielectric materials leads to intensity-independent optical phases, which helps to preserve coherence during propagation.

In order to cover all photonics functionalities, i.e., from generation to detection through manipulation of either classical or quantum light, both platforms are required. The most powerful and attractive devices will combine different material platforms, either in the form of hybrid or heterogeneous integration. Examples of hybrid and heterogeneous integration have been reported in the recent years [14, 15], showing both the promises and the challenges of this approach. Reaching optimum control and knowledge of each platform separately is the key to enable a successful merging of the approaches. However, due to the required large-scale facilities and scientific and technological background knowledge specific to each platform, researchers typically focus on one of the platforms. In this thesis we focus on exploring the capabilities of a dielectric material platform that provides silicon-nitride optical waveguides, because the locally available state-of-the-art fabrication facilities and research environment offer promising progress.

Within the dielectric integrated optical waveguides, silicon-based material platforms, i.e., Silicon-on-Insulator (SOI), Silicon nitride $\left(\mathrm{Si}_{3} \mathrm{~N}_{4}\right)$ or Silica-on-Silicon (SoS), are the most widely used platforms for photonic integrated circuits due to the high compatibility of their fabrication processes with integrated electronic circuits, i.e., these platforms are CMOS compatible. These platforms all allow a large variety of optical components but present complementary properties and strengths.

Amongst them, the SoS platform presents very low propagation loss in straight waveguide sections (due to low material absorption and scattering at roughness) but it suffers from high radiative loss in curved waveguide sections (part of the light losing its guiding). The latter makes space-efficient implementation of integrated optical 
components difficult, thereby forming an obstacle for implementing high complexity at a given size of a chip.

A high component-density is instead achievable in SOI waveguide circuits. The key strength of this platform is the high compatibility with active components such as detectors and high-speed optical modulators via extraction or injection of charge carriers, even though this leads to increased propagation losses due to free-carrier absorption. SOI-based waveguides show a high-index contrast (3.5 in the core versus 1.5 in the cladding) that enables high-component density thanks to low radiative loss even at small bending radii, i.e., down to $2 \mu \mathrm{m}$. The transparency range of the SOI platform is in the near-infrared (from 1.1 to $3.7 \mu \mathrm{m}$ ) and propagation losses are typically within 1 and $2 \mathrm{~dB} / \mathrm{cm}$.

$\mathrm{Si}_{3} \mathrm{~N}_{4}$-based waveguides offer a large variety of passive components for linear and non-linear optical applications or for approaches based on hybrid integration [14]. The index contrast of $\mathrm{Si}_{3} \mathrm{~N}_{4}$-based waveguides, i.e., 2 (core) versus 1.5 (cladding), is somewhat smaller than in SOI such that the typical smallest bending radii where radiative (curvature) loss is not dominant, lies in the range of a few tens of micrometers. The maximum density of components is therefore slightly lower than with SOI. On the other hand the somewhat lower index contrast, and also the fact that $\mathrm{Si}_{3} \mathrm{~N}_{4}$ is glass-like and smooth (as compared to etched crystalline Si structures) reduces scattering loss due to sidewall roughness at the core-cladding interfaces. Another advantage of the somewhat lower contrast is a weaker mode confinement in the transverse dimensions. This renders the effective refractive index of the waveguide less sensitive to fabrication imperfections of the waveguide width. The result is that $\mathrm{Si}_{3} \mathrm{~N}_{4}$-based circuits can achieve the intended functionalities via high fidelity and reproducibility in fabrication more likely than in SOI devices.

The great advantage of the $\mathrm{Si}_{3} \mathrm{~N}_{4}$ platform is that low loss can be achieved in spite of a moderately high index contrast. Low loss can already be proved by the great spectral range where the waveguides are transparent, reaching coarsely across the entire visible, continuing into the near-infrared, i.e., from $400 \mathrm{~nm}$ to $2.7 \mu \mathrm{m}$, limited by the absorption of the silica cladding [16]. Transparency up to blue wavelengths (photon energy $3 \mathrm{eV}$ at $400 \mathrm{~nm}$ ) is indicative for the large electronic bandgap of the involved dielectrics ( 5 and $8 \mathrm{eV}$ for core and cladding, respectively). At near-infrared wavelengths in the telecom range (e.g. at $1.55 \mu \mathrm{m}, 0.8 \mathrm{eV}$ ) $\mathrm{Si}_{3} \mathrm{~N}_{4}$ typically allows propagation loss lower than that of SOI. Using optimized fabrication strategies in connection with dedicated cross section design has been used to demonstrate losses as low as $0.1 \mathrm{~dB} / \mathrm{m}(0.001 \mathrm{~dB} / \mathrm{cm})[17]$. 


\section{Introduction}

Based on the named properties, the $\mathrm{Si}_{3} \mathrm{~N}_{4}$ platform covers a highly attractive region in terms of bandgap energy and fabrication technology. Because of low propagation loss, moderately high index contrast and uncritical fabrication of circuits with desired optical parameters dense fabrication of components for high functionality can be achieved. These properties match perfectly with the central demands of emerging programmable integrated photonic circuits.

A majority of the photonic integrated circuits realized so far, either based on simple or complex circuitries, are designed to perform rather specific functions that require no or only little external control or programmability. Examples are tunable ring resonators and Mach-Zehnder interferometers for, e.g., optical filters, delay lines, (de-)multiplexers, feedback circuits for integrated hybrid lasers, wavelength selective reflectors and sources of correlated photons.

Only recently have integrated circuits moved towards providing a wider, more general set of functionalities. This is achieved realizing extensive programmability in combination with complex circuitry, similar to the so-called Field Programmable Gate Arrays (FPGAs) in electronics. An example of this is programmable microwave photonic filters in the form of resonator networks [18, 19]. Other examples are reconfigurable photonic integrated circuits, also called photonic processors, which function by using a mesh of externally tunable interferometers and phase shifters [9, 18-20]. The latter type of processor can be considered universal in the sense of enabling any unitary amplitude and phase transformation of light distributed across a multitude of input ports to a multitude of output ports. Depending on the topology and arrangement of the mesh, i.e., squared [18, 21], triangular [19, 22] or hexagonal [19], hundreds of functionalities can be demonstrated [7, 19].

With the escalating complexity of such processors, the intended performance and functionality becomes increasingly attractive and programmable photonic processors are enriching various fields such as microwave photonics and quantum information processing. Since 2015, impressive steps have been made and large processors with tens of spatial modes and hundreds of components have already been demonstrated. However, performance and functionality have also become more critically dependent on the precision of fabrication and calibration. In fact, fabrication imperfections, optical losses and the control of circuits as desired have gained an increasing and even dominant importance, despite significant progress in concepts and demonstrations.

Various methods have been presented for obtaining (close-to-) ideal performance from an imperfect photonic integrated circuit such as, numerical optimization and addition of extra (also imperfect) components [23-25]. However, basing a processor on 
a large number of imperfect components, or on components with unknown performance, makes it difficult to predict the output of a processor, while most functionalities require predictable results in processing. As a bypassing alternative, self-configuring protocols have been proposed and demonstrated [26-28]: these protocols aim to avoid the characterization of the processor while progressively optimizing the desired functionality.

Lately, reconfigurable photonic circuits have been shown to be a very promising platform for artificial neural networks, which perform part of the training process of the neurons optically and thus more efficiently than on an electronic computer. For instance, a neural network involving linear photonic circuits as part of their functionality has been demonstrated for simple vowel recognition [20]. Given the already high complexity of photonic processors and the consequent difficulty that fabrication imperfections induce in the predictability of their optical response, neural networks can actually be exploited even in the calibration of these integrated photonic devices [29].

In this thesis we concentrate on $\mathrm{Si}_{3} \mathrm{~N}_{4}$-based reconfigurable photonic integrated circuits with low-loss propagation, to explore interference in the spectral and temporal domain for advanced applications. We investigated two types of integrated interferometric devices featuring low loss in combination with programmability for classical and quantum photonic processing. The first is simple tunable microring resonator circuits in combination with neural network data processing for the analysis of classical light in the spectral domain as wavelength meter. The second is a complex tunable network of waveguide interferometers for controlling quantum correlations (coincidences) between single photons. Both devices derive their attractive properties from low-loss, high-index contrast and phase-programmable integrated waveguide circuits.

In Chapter 2 we recall certain theoretical aspects relevant for the photonic building blocks investigated in this thesis, i.e., ring resonators and Mach-Zehnder interferometers, and we recall the description of basic linear optical circuits for quantum information processing. In Chapter 3 we investigate the combination of photonic integrated resonator circuits and a so-called smart readout, an optimization algorithm based on a neural network, for achieving a high-precision wavelength meter on-chip. Chapter 4 presents the characterization of the largest reconfigurable photonic processor realized in $\mathrm{Si}_{3} \mathrm{~N}_{4}$ so far. We study, for the first time, the extent to which the optimization of phase reconfiguration can improve the functionalities of a real processor in spite of imperfections due to fabrication. In Chapter 5 we report 
1. Introduction

experimental results on how the processor can be exploited for quantum information processing. 


\section{References}

[1] Thylén, L. and L. Wosinski, "Integrated photonics in the 21st century". Photonics Research 2(2), p. 75-81 (2014).

[2] Washburn, A.L., et al., "Multiplexed cancer biomarker detection using chipintegrated silicon photonic sensor arrays". Analyst 141(18), p. 5358-5365 (2016).

[3] Qu, X., et al., "Nanotechnology for a Safe and Sustainable Water Supply: Enabling Integrated Water Treatment and Reuse". Accounts of Chemical Research 46(3), p. 834-843 (2013).

[4] Luo, D.H., et al., "An integrated photonic sensor for in situ monitoring of hazardous organics". Sensors and Actuators B: Chemical 92(1), p. 121-126 (2003).

[5] Hofmann, O., et al., "Monolithically integrated dye-doped PDMS long-pass filters for disposable on-chip fluorescence detection". Lab on a Chip 6(8), p. 981987 (2006).

[6] Chovan, J. and F. Uherek, "Photonic Integrated Circuits for Communication Systems". 27, p. 357-363 (2018).

[7] Marpaung, D., J. Yao, and J. Capmany, "Integrated microwave photonics". Nature Photonics 13(2), p. 80-90 (2019).

[8] Koos, C., et al. "Photonic Integration for Metrology and Sensing". in Advanced Photonics 2017 (IPR, NOMA, Sensors, Networks, SPPCom, PS). New Orleans, Louisiana (Optical Society of America 2017), paper ITh1A.1.

[9] Carolan, J., et al., “Universal linear optics”. Science 349(6249), p. 711 (2015).

[10] Broome, M.A., et al., "Photonic Boson Sampling in a Tunable Circuit". Science 339(6121), p. 794 (2013).

[11] Crespi, A., et al., "Integrated multimode interferometers with arbitrary designs for photonic boson sampling". Nature Photonics 7, p. 545 (2013).

[12] Spring, J.B., et al., "Boson Sampling on a Photonic Chip". Science 339(6121), p. 798 (2013).

[13] Tillmann, M., et al., “Experimental boson sampling”. Nature Photonics 7, p. 540 (2013).

[14] Fan, Y., et al. "290 Hz Intrinsic Linewidth from an Integrated Optical Chipbased Widely Tunable InP-Si $\mathrm{N}_{4}$ Hybrid Laser". in Conference on Lasers and Electro-Optics. San Jose, California (Optical Society of America 2017), paper JTh5C.9. 
1. References

[15] Oulton, R.F., et al., "A hybrid plasmonic waveguide for subwavelength confinement and long-range propagation". Nature Photonics 2, p. 496 (2008).

[16] Muñoz, P., et al., "Silicon Nitride Photonic Integration Platforms for Visible, Near-Infrared and Mid-Infrared Applications". Sensors 17, 2088 (2017).

[17] Bauters, J.F., et al., "Planar waveguides with less than $0.1 \mathrm{~dB} / \mathrm{m}$ propagation loss fabricated with wafer bonding". Optics Express 19(24), p. 24090-24101 (2011).

[18] Zhuang, L., et al., "Programmable photonic signal processor chip for radiofrequency applications". Optica, 2(10) p. 854-859 (2015).

[19] Pérez, D., et al., "Multipurpose silicon photonics signal processor core”. Nature Communications 8(1), p. 636 (2017).

[20] Shen, Y., et al., "Deep learning with coherent nanophotonic circuits". Nature Photonics 11, p. 441 (2017).

[21] Clements, W.R., et al., “Optimal design for universal multiport interferometers". Optica 3(12), p. 1460-1465 (2016).

[22] Reck, M., et al., "Experimental realization of any discrete unitary operator". Physical Review Letters 73(1), p. 58-61 (1994).

[23] Mower, J., et al., "High-fidelity quantum state evolution in imperfect photonic integrated circuits". Physical Review A 92(3), p. 032322 (2015).

[24] Miller, D.A.B., "Perfect optics with imperfect components". Optica 2(8), p. 747750 (2015).

[25] Burgwal, R., et al., "Using an imperfect photonic network to implement random unitaries". Optics Express 25(23), p. 28236-28245 (2017).

[26] Miller, D.A.B., "Self-aligning universal beam coupler". Optics Express 21(5), p. 6360-6370 (2013).

[27] Miller, D.A.B., "Self-configuring universal linear optical component". Photonics Research 1(1), p. 1-15 (2013).

[28] Ribeiro, A., et al., “Demonstration of a $4 \times 4$-port universal linear circuit". Optica 3(12), p. 1348-1357 (2016).

[29] Zhou, H., et al., "Self-learning photonic signal processor with an optical neural network chip". arXiv:1902.07318 (2019). 


\section{Theoretical background}

In this chapter, we recall selected sections in the theoretical description of integrated linear optics, quantum information and neural networks, for later reference and selfcontained readability.

\subsection{Basics of waveguide optics}

In this thesis, integrated optical waveguides made from two dielectric materials are the basic elements, as shown schematically in Fig. 2.1(a). Specifically, we use as the core material stoichiometric silicon nitride due to its relatively high refractive index
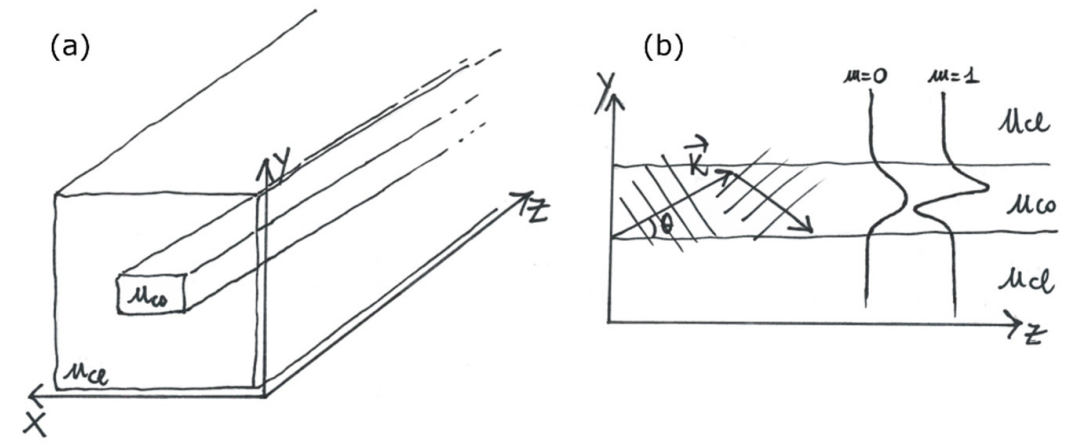

Fig. 2.1 (a) Schematic of a dielectric optical waveguide of cross-section in the xy-plane and propagation direction along the $\mathrm{z}$-axis. The dielectric waveguide core of refractive index $n_{c o}$ is surrounded by a cladding of refractive index $n_{c l}$. (b) Side view of an optical waveguide. The interference of a plane wave, of wavevector $\vec{k}$, travelling under an angle $\theta$ with its reflection at the core boundaries determines the field distribution. The field distribution of the fundamental and first-order modes is displayed.

$\left(\mathrm{n}_{\mathrm{co}} \approx 2\right)$, embedded in a cladding of silicon oxide with lower index $\left(\mathrm{n}_{\mathrm{cl}} \approx 1.5\right)$. The advantage of this choice of materials and the associated fabrication technology, summarized under the term silicon nitride waveguide platform, is that extremely low optical loss can be achieved [1] and that a large variety of different cross sections can be fabricated [2]. The latter allows the adjustment of the field cross section of the guided modes, which is essential for minimizing the propagation loss in waveguides with varying curvature. The variety of cross sections further allow the reduction of loss in coupling to external glass fiber connections, and the restriction of propagation to a single spatial mode of desired polarization. Accordingly, the design of a waveguide circuit starts with choosing the desired cross sectional size of the optical field. This is done via selecting a certain cross section as an initial guess, followed by numerical calculation of the resulting 


\section{Theoretical background}

field and subsequent readjustments of the cross section, to obtain the best match of the mode field to the desired size and shape.

The field distribution of guided modes in an optical waveguide of specific geometry with stepwise uniform refractive index profile is found by solving the Helmholtz or wave equation

$$
\nabla^{2} \overrightarrow{\mathrm{E}}(\overrightarrow{\mathrm{r}})+\mathrm{k}^{2} \overrightarrow{\mathrm{E}}(\overrightarrow{\mathrm{r}})=0, \mathrm{k}=\mathrm{n}(\mathrm{x}, \mathrm{y}) \cdot \mathrm{k}_{0},
$$

where $\vec{E}(\vec{r})=\vec{E}(x, y) e^{-j \beta z}$ is a transverse electric field propagating longitudinally along the $\mathrm{z}$-axis with $\beta$ being the propagation constant. The transverse refractive index distribution $\mathrm{n}(\mathrm{x}, \mathrm{y})$ is imposed by the core and cladding cross sectional geometry and materials and $\mathrm{k}_{0}$ is the light propagation constant in vacuum defined as $\mathrm{k}_{0}=2 \pi / \lambda_{0}{ }^{\prime}$ with $\lambda_{0}$ the field vacuum wavelength. In general, to find the two-dimensional optical mode field distribution $\mathrm{E}(\mathrm{x}, \mathrm{y})$ and its propagation constant for a given twodimensional index distribution, Eq. (1.1) needs to be solved numerically. For certain geometries of higher symmetry, approximate analytical method have been devised, such as by Marcatili [3] and Hocker and Burns [4]. These methods separate the twodimensional problem of determining $\vec{E}(x, y)$ into two one-dimensional problems, i.e., solving the Helmholtz equation for a slab waveguide in both transversal directions for the one-dimensional index distributions $\mathrm{n}(\mathrm{x})$ and $\mathrm{n}(\mathrm{y})$ and then combine the solutions. As described by Saleh and Teich [5], the solution of Eq. (1.1) for a slab waveguide is of the form of a monochromatic plane wave

$$
E_{x}(y, z)=e^{-j k_{y} \cdot y} e^{-j k_{z} \cdot z},
$$

with $\mathrm{k}_{\mathrm{y}}=\mathrm{n}_{\mathrm{co}} \mathrm{k}_{0} \sin \theta$ and $\mathrm{k}_{\mathrm{z}}=\mathrm{n}_{\mathrm{co}} \mathrm{k}_{0} \cos \theta$, where $\theta$ is the propagation angle between the wave vector and the z-axis (Fig. 2.1(b)) and where $E_{x}(y, z)$ means that we are considering a waveguide of infinite width in the $x$ direction.

Imposing the condition of constructive interference in the propagation direction between the original plane wave and its reflections at the waveguide dielectric boundaries, the propagation angles and constants become quantized to discrete values, i.e., $\theta_{\mathrm{m}}, \mathrm{k}_{\mathrm{ym}}$ and $\mathrm{k}_{\mathrm{zm}}$, where $\mathrm{m}=0$ indicates the fundamental mode and $\mathrm{m} \geq 1$ are higher-order modes. The interference of the travelling TEM plane wave at angle $+\theta$ with its reflection at angle $-\theta$, defines the transverse field distribution $E_{x}(y, z)$ that propagates along the $\mathrm{z}$-axis

$$
E_{x}(y, z)=c_{m}(d) \cdot u_{m}(y) e^{-j \beta_{m} z}
$$




$$
\mathrm{u}_{\mathrm{m}}(\mathrm{y}) \propto \begin{cases}\cos \left(\frac{2 \pi \sin \theta_{\mathrm{m}}}{\lambda} \mathrm{y}\right) & \mathrm{m}=0,2, \ldots \\ \sin \left(\frac{2 \pi \sin \theta_{\mathrm{m}}}{\lambda} \mathrm{y}\right) & \mathrm{m}=1,3, \ldots\end{cases}
$$

with $\mathrm{u}_{\mathrm{m}}(\mathrm{y})$ normalized functions, $\mathrm{c}_{\mathrm{m}}(\mathrm{d})$ constant and $\beta_{\mathrm{m}}=\mathrm{n}_{\mathrm{co}} \mathrm{k}_{0} \cos \theta_{\mathrm{m}}$. In Fig. 2.1(b) the field distributions of the fundamental and first-order mode are reported.

A guided optical mode does not vanish abruptly at the boundaries of the waveguide core: its light field penetrates in the surrounding cladding decaying, however, exponentially as $\mathrm{e}^{ \pm \gamma_{\mathrm{m}} \mathrm{y}}$, where $\gamma_{\mathrm{m}}$ is the extinction coefficient. This is important for two reasons. Firstly, higher-order modes have a greater propagation angle and a lower extinction coefficient, meaning that higher-order modes penetrate deeper in the cladding and will experience higher radiation losses in curved waveguide sections than the fundamental mode. This enables the restriction of the light flow in waveguide circuits to a single mode even if the coupling of light into the entrance of a waveguide had partly excited higher-order modes. Secondly, making use of the evanescent field allows the coupling of light from one waveguide to the next waveguide, if that one is sufficiently closely spaced along a sufficiently long propagation distance. The optical-power-transfer dynamics between these two waveguides, that are said to form a directional coupler, is described well by coupledmode theory [5]. At a given light frequency, an adjustable amount of power can be transferred with such a coupler, depending of the spacing and propagation distance chosen in the waveguide circuit design. For instance, to realize a waveguide MachZehnder interferometer, one would require two couplers each providing $50 \%$ power splitting, while coupling light into high-quality waveguide resonators requires directional couplers with small power splitting.

Due to the transverse amplitude distribution and the evanescent tails of the optical mode into the cladding, different parts of a guided mode propagate through different materials experiencing, thus experiencing a different refractive index. The mode as an entity will then propagate with the so-called effective refractive index, $\mathrm{n}_{\mathrm{eff}}=\frac{\beta_{\mathrm{m}}}{\mathrm{k}_{0}}$, which is given by a weighted average of the refractive indices of the involved materials, where the weighting factors are given by the fraction of light confined into that particular material,

$$
\mathrm{n}_{\mathrm{eff}} \sim \sum_{\mathrm{i}} \Gamma_{\mathrm{i}} \mathrm{n}_{\mathrm{i}}, \quad \quad \Gamma_{\mathrm{i}}=\frac{\int_{0}^{\mathrm{d} / 2} \mathrm{u}_{\mathrm{m}}^{2}(\mathrm{y}) \mathrm{dy}}{\int_{0}^{\infty} \mathrm{u}_{\mathrm{m}}^{2}(\mathrm{y}) \mathrm{dy}}
$$

with $\Gamma_{\mathrm{i}}$ confinement factor defined as the ratio of the optical power contained in the slab and the total optical power. In this way, the waveguide cross section and mode field distribution determine the optical length of waveguide circuits, for instance the 


\section{Theoretical background}

optical length of waveguide resonators and the optical arm length in waveguide MachZehnder interferometers.

\subsubsection{Thermal tuning}

An absolutely essential ingredient in programmable routing of light through optical waveguide circuits, or for tuning the transmission of resonators, is that the phase velocity along certain waveguide sections can be externally adjusted, via adjusting the effective refractive index, $n_{\text {eff. }}$ A well-known method to achieve a change of $n_{e f f}$ is the local heating of the waveguide as its core and cladding refractive index $n_{c o}$ and $n_{c l}$ are temperature dependent as described by the thermo-optic coefficients $\frac{\mathrm{dn}_{\mathrm{co}}}{\mathrm{dT}}$ and $\frac{\mathrm{dn}_{\mathrm{cl}}}{\mathrm{dT}}$. The thermal expansion of the material is significantly smaller than the thermo-optic effect and is therefore not considered here. The effective refractive index is itself temperature dependent as described by

$$
\mathrm{n}_{\mathrm{eff}}(\mathrm{T})=\Gamma_{\mathrm{co}} \mathrm{n}_{\mathrm{co}}(\mathrm{T})+\Gamma_{\mathrm{cl}} \mathrm{n}_{\mathrm{cl}}(\mathrm{T})
$$

where $n_{c o}(T)$ and $n_{c l}(T)$ can be calculated from their thermo-optic coefficient for a desired temperature.

For the waveguides used here $\left(\mathrm{Si}_{3} \mathrm{~N}_{4}\right.$ core and $\mathrm{SiO}_{2}$ cladding fabricated with lowpressure chemical vapor deposition (LPCVD) or Si wafer oxidation, respectively) the thermo-optic coefficients [6] are given as:

$$
\begin{aligned}
& \frac{\mathrm{dn}_{\mathrm{Si}_{3} \mathrm{~N}_{4}}}{\mathrm{dT}}=(2.45 \pm 0.09) \times 10^{-5}\left(\frac{\mathrm{RIU}}{{ }^{\circ} \mathrm{C}}\right), \\
& \frac{\mathrm{dn}_{\mathrm{SiO}_{2}}}{\mathrm{dT}}=(0.95 \pm 0.10) \times 10^{-5}\left(\frac{\mathrm{RIU}}{{ }^{\circ} \mathrm{C}}\right) .
\end{aligned}
$$

Integrating the thermo-optic coefficients we obtain

$$
\begin{gathered}
\mathrm{n}_{\mathrm{Si}_{3} \mathrm{~N}_{4}}(\mathrm{~T})=\mathrm{n}_{\mathrm{Si}_{3} \mathrm{~N}_{4}}\left(\mathrm{~T}_{0}\right)+\frac{\mathrm{dn}_{\mathrm{Si}_{3} \mathrm{~N}_{4}}}{\mathrm{dT}} \mathrm{dT} \\
\mathrm{n}_{\mathrm{SiO}_{2}}(\mathrm{~T})=\mathrm{n}_{\mathrm{SiO}_{2}}\left(\mathrm{~T}_{0}\right)+\frac{\mathrm{dn}_{\mathrm{SiO}_{2}}}{\mathrm{dT}} \mathrm{dT}
\end{gathered}
$$

with $\mathrm{dT}=\mathrm{T}-\mathrm{T}_{0}$, and $\mathrm{T}_{0}$ the room temperature. For the optical structures described in this thesis (Chapter 3) we obtain that a temperature variation of $\sim 195{ }^{\circ} \mathrm{C}$ induces a $2 \pi$ phase shift on a ring resonator of radius $r \sim 85 \mu \mathrm{m}$, corresponding to a wavelength shift of $\sim 14 \frac{\mathrm{pm}}{{ }^{\circ} \mathrm{C}}$. This is well in accordance with results reported in literature [6]. The exact value of wavelength (or phase) shift, ultimately depends on the waveguide crosssection as it defines the overlap between the optical field and thermal gradient. 
The temperature change of a waveguide refractive index is realized by local resistive heating of a metal layer stripe, called heater or thermo-optic phase shifter, deposited on the top cladding in correspondence of the waveguide where the length of the metal stripe is along the waveguide propagation direction. When a voltage difference $\mathrm{V}$ is applied across the heater, an electrical current $\mathrm{I}=\mathrm{V} / \mathrm{R}$ starts flowing, thanks to the resistivity $\mathrm{R}$ of the metal layer. The passage of current through $\mathrm{a}$ conductor produces resistive heat, proportional to the electrical power generated $\mathrm{P}=$ $\mathrm{V}^{2} / \mathrm{R}$. The temperature variation $\mathrm{dT}$ associated with the current flow can be determined as follow $\mathrm{dT}=\frac{\mathrm{P} \cdot \mathrm{t}}{\mathrm{m} \cdot \mathrm{c}} \propto \mathrm{V}^{2}$, where $\mathrm{t}$ is the time, $\mathrm{m}$ is the mass of the heater and $\mathrm{c}$ is the heat capacity of the metal used as heater. The heat generated on the top cladding of the waveguide propagates through the cladding reaching thus the waveguide and induce a temperature variation of the core and cladding material. As described above, such temperature variation causes a refractive index change that ultimately induces a phase delay of a light wave propagating in the waveguide given by $\theta=\mathrm{k} \cdot \mathrm{L} \cdot \mathrm{dn}_{\mathrm{eff}}=$ $\frac{2 \pi}{\lambda} \cdot \mathrm{L} \cdot\left(\mathrm{n}_{\mathrm{eff}}(\mathrm{T})-\mathrm{n}_{\mathrm{eff}}\left(\mathrm{T}_{0}\right)\right) \propto \frac{2 \pi}{\lambda} \cdot \mathrm{L} \cdot \frac{\mathrm{dn} \mathrm{eff}_{\mathrm{ef}}}{\mathrm{dT}} \cdot \mathrm{dT}$, where $\lambda$ is the wavelength, $\mathrm{L}$ is the distance travelled along the waveguide and $\mathrm{dT}$ is the temperature variation. Expressing $\mathrm{dT}$ by resistive heating, we find the phase delay to be $\theta=c+d V^{2}$, where $\mathrm{c}$ and $\mathrm{d}$ correspond to the offset of the phase delay, given by an offset in L, and to how fast the phase delay varies with the heating voltage, respectively. Characterization of the phase delay induced by a thermo-optic phase shifter for a MZI will be extensively treated in Chapter 4.

\subsubsection{Optical modes for channel waveguides}

A powerful way of solving Eq. 1.1 for arbitrary cross-section geometries is using the Finite Element (FE) Method which divides the domain of calculation into smaller subdomains over which the wave equation has an exact solution. In this thesis we deploy such FE calculations via readily available software (COMSOL Multiphysics ${ }^{\circledR}$ ) to calculate and optimize the optical mode field distributions for diverse waveguide cross-sections for single-mode operation at a central wavelength of $1550 \mathrm{~nm}$. In the following we report, as an overview and comparison, the transverse intensity distribution for the fundamental mode of the three different waveguide cross-sections as employed in this thesis (see Fig. 2.2). 


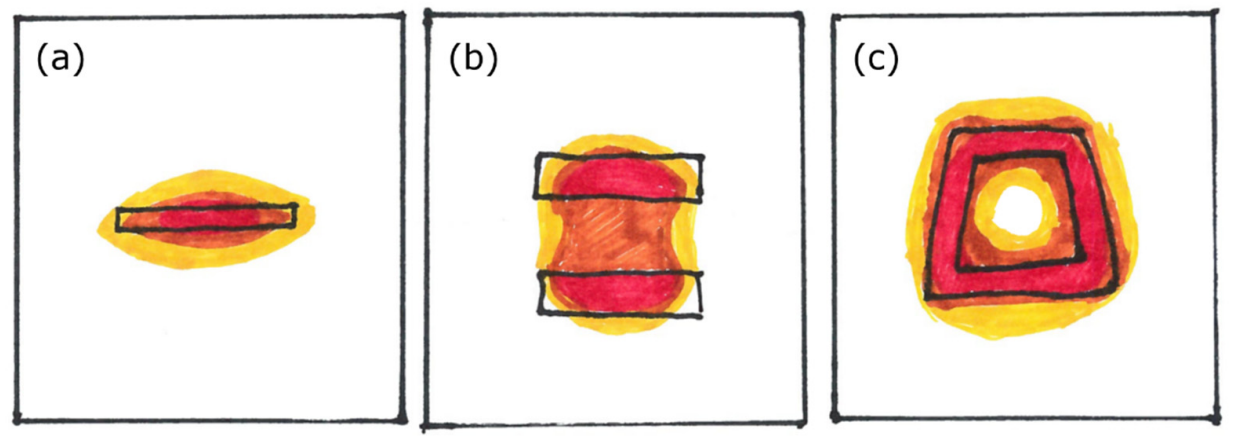

Fig. 2.2 Mode field distributions for different waveguide cross-sections. (a) Single stripe width $=2.5 \mu m(\mathrm{w})$, thickness $=120 \mathrm{~nm}$, (b) Symmetric double stripe $\mathrm{w}=1.2 \mu \mathrm{m}, \mathrm{t}=170 \mathrm{~nm}$, distance between stripes $\mathrm{d}=500 \mathrm{~nm},(\mathrm{c})$ Box-shaped $\mathrm{SiO}_{2}$ core $0.5 \times 0.5 \mu \mathrm{m}^{2}$, thickness $\mathrm{Si}_{3} \mathrm{~N}_{4} 170 \mathrm{~nm}$. The refractive index of core and cladding considered for calculations are $n_{S_{3} N_{4}}=1.977 \pm 0.003$ and $n_{S_{i O}}=1.428 \pm 0.011$, respectively.

Depending on the envisioned function, these waveguide cross sections provide specific properties, as described in the following text. Choosing a high width-tothickness (aspect) ratio as in Fig. 2.2(a) enables lower propagation losses as dominant scattering from vertical sidewalls (material boundary defined by lithography) is minimized. The horizontal material interfaces usually show very low scattering loss because the material boundary is very smooth (defined by deposition rate). Such highaspect-ratio design, due to its relatively small core cross sectional area, leads, however, to weak confinement of the fundamental optical mode (large mode field diameter, MFD). The consequence is then a relatively high propagation loss in waveguides with curvature radii typically below $\sim 365 \mu \mathrm{m}$ bend radius for the shown cross section. Highaspect-ratio waveguides are preferable when low propagation losses are needed, e.g., for ultrahigh quality factor ring resonators (Chapter 3), although the drawback is a low density of components on the chip. A related effect imposed by the strong asymmetry of the core is a strong birefringence and thus also a strong difference in radiation loss at bent waveguide sections. This helps to maintain the polarization of light in a single state, having its electric field oriented horizontally in Fig. 2.2(a), in a so-called transverse electric (TE) mode. This effect is beneficial if a well-defined transmission spectrum is required, for instance, in spectrometric applications of waveguide interferometers and resonators.

In order to enable smaller bend radii, the waveguide core cross sectional area must be increased to provide stronger optical confinement of the light to the core. However, simply increasing the area with a thicker waveguide core (i.e. lower aspect ratio) also increases the propagation losses due to a larger contribution of scattering from vertical side walls. Similarly, because $\mathrm{Si}_{3} \mathrm{~N}_{4}$ possesses intrinsically higher material propagation 
loss (due to a smaller bandgap than $\mathrm{SiO}_{2}$ and also fabrication conditions) simply increasing the core thickness is not the best choice.

Instead, clever countermeasures can be taken to achieve both a high confinement and low propagation losses. Two examples are shown in Fig. 2.2(b) and 2.2(c) where the waveguide cores, named respectively symmetric double stripe (SDS) and box-shaped, are designed in such a way that the most of the optical power overlaps with the lowerloss $\mathrm{SiO}_{2}$ cladding and where the length of vertical sidewalls is minimized (SDS). The SDS waveguides can especially enable relatively low propagation losses $(\sim 0.1 \mathrm{~dB} / \mathrm{cm})$ in combination with relatively tight bend radii (down to $\sim 80 \mu \mathrm{m}$ ). There is also a version of the double stripe cross section where the top stripe is thicker than the bottom one. Local adiabatic tapering down of the top stripe guides the light fully into the remaining lower, high-aspect-ratio stripe. This allows for reduced propagation loss in intermediate straight sections (as in Fig. 2.2(a)) and for sharp curvature (as in Fig. 2.2(b)), to enable a high-component density simultaneously with overall low loss propagation losses, as needed for implementing large linear optical processors. A second advantage of such tapering is that spot size converters can be implemented at the ends of waveguides, at the edges of the chip, for low-loss fiber coupling.

\subsection{Photonic building blocks}

In order to provide various on-chip photonic functionalities this thesis makes use of a number of well-known integrated optical waveguide components, also called waveguide building blocks. For later reference we recall the functional description of the two main building blocks used here, which are the waveguide ring resonator, also called microring resonator (RR), and the waveguide Mach-Zehnder interferometer (MZI). These building blocks enable more complex architectures to be devised, as employed in chapters 3,4 and 5 of the thesis, i.e., sequential RRs (so-called Vernier configuration) and entire networks made of MZIs.

\subsubsection{Waveguide Ring Resonator}

An integrated ring resonator is a travelling wave cavity which has found many applications, e.g., as high-quality spectral filters and tunable delay lines in integrated microwave photonics [7] for spectroscopic analysis [8-10] or for resonant enhancement of the light intensity to drive non-linear processes of light generation [11, 12].

Following the extensive description of Madsen and Zhao [13] we consider here the representative example of a so-called add-drop RR (Fig. 2.3 left), which consists of two 


\section{Theoretical background}

straight bus waveguides that are placed tangential to the ring resonator to form two directional couplers indicated with the dashed-line regions. The resonator is schematically chosen to have a circular shape with a circumference $L=2 \pi r$, with $r$ the radius, although often these resonators are fabricated with a race-track-like shape for higher flexibility in design and for increasing the component density.

The transmission of monochromatic light through such resonator can be described via the field-coupling coefficients of the two directional couplers and the optical phase shift of the light experienced in a full roundtrip (the latter is given by the resonator

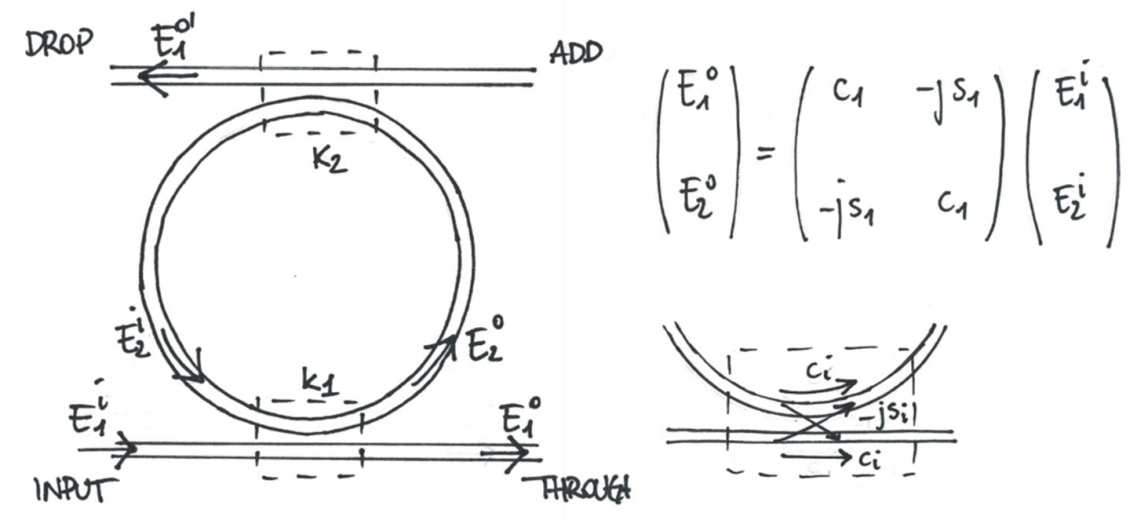

Fig. 2.3 Schematic of an add-drop ring resonator, definition of the electric field amplitude components and transfer matrix of the bottom directional coupler of power coupling ratio $k_{1}$. The top directional coupler $k_{2}$ is defined analogously.

length and effective waveguide index). The field components generated by the $i^{\text {th }}$ directional coupler $(i=1$ or 2$)$ are given by the self-coupling coefficients $c_{i}=\sqrt{1-k_{i}}$ and the cross-coupling coefficient $s_{i}=\sqrt{k_{i}}$ (as indicated at the rhs of Fig. 2.3 with arrows crossing the waveguide couplers), where $\mathrm{k}_{\mathrm{i}}$ is the according power coupling coefficient. Provided that losses can be neglected, the coupling coefficients satisfy the following relation $|c|^{2}+|s|^{2}=1$.

Once light is coupled into the ring via the bottom coupler, i.e., $E_{2}^{0}=-j s_{1} E_{1}^{i}$, it travels along its circumference, and part of it returns to the bottom directional coupler, modified to a value of $\mathrm{E}_{2}^{\mathrm{i}}=\mathrm{c}_{2} \mathrm{te}^{-\mathrm{j} \theta} \mathrm{E}_{2}^{\mathrm{o}}$, where $\mathrm{t}$ and $\theta$ are the round-trip power transmission and phase delay, respectively, and $c_{2}$ is the self-coupling coefficient of the top directional coupler. In terms of the propagation loss coefficient $\gamma[\mathrm{dB} / \mathrm{cm}]$ of the waveguide that forms the ring resonator, we find $t=10^{-\frac{\gamma \cdot \mathrm{L}}{10}}$ (i.e., $t<1$ due to propagation loss). The round-trip phase delay is found as $\theta=\mathrm{L} \beta(\lambda)=\mathrm{Lkn}_{\mathrm{eff}}$, with $\mathrm{k}=$ $\frac{2 \pi}{\lambda}$. The through-port output field will be then given by $\mathrm{E}_{1}^{\mathrm{o}}=-\mathrm{js} \mathrm{E}_{2}^{\mathrm{i}}$. Only certain wavelengths acquire a round-trip phase delay of $\theta=\mathrm{m} 2 \pi$ ( $\mathrm{m}$ is an integer number) such as to interfere destructively with the bypassed (through) fraction of the input 
travelling plane wave $E_{1}^{i}$, i.e., $m \lambda_{\text {res }}=n_{\text {eff }} \mathrm{L}$. As a result, the through-port transmission function exhibits a minimum at each resonant wavelength, whereas there is maximum transmission to the drop port for resonant wavelengths.

In a ring resonator, light can perform many round trips limited only by the propagation loss inside the ring and the value of the cross-coupling coefficients. In order to derive the overall output response of a ring resonator over all roundtrips it is required to superimpose the infinite sum of the delayed versions of the input field weighted by the round-trip cavity transmission,

$$
\mathrm{H}_{\text {through }}=\frac{\sum \mathrm{E}_{1}^{\mathrm{o}}}{\mathrm{E}_{1}^{\mathrm{i}}}, \mathrm{H}_{\mathrm{drop}}=\frac{\sum \mathrm{E}_{1}^{\mathrm{o}}}{\mathrm{E}_{1}^{\mathrm{i}}}
$$

where

$$
\begin{array}{ll}
\mathrm{H}_{\text {through }}=\mathrm{c}_{1}-\mathrm{s}_{1}^{2} \mathrm{c}_{2} \mathrm{te}^{-\mathrm{j} \theta}\left(1+\mathrm{x}+\mathrm{x}^{2}+\cdots\right), & \mathrm{x}=\mathrm{c}_{1} \mathrm{c}_{2} \mathrm{te}^{-\mathrm{j} \theta} \\
\mathrm{H}_{\text {drop }}=\mathrm{s}_{1} \mathrm{~s}_{2} \sqrt{\mathrm{te}^{-\mathrm{j} \theta}}\left(1+\mathrm{x}+\mathrm{x}^{2}+\cdots\right), & \mathrm{x}=\mathrm{c}_{1} \mathrm{c}_{2} \mathrm{te}^{-\mathrm{j} \theta} .
\end{array}
$$

Considering that $|x|<1$, the infinite power series converge to $\frac{1}{1-x}$ and thus the transfer functions can be further simplified to

$$
\mathrm{H}_{\text {through }}=\frac{\mathrm{c}_{1}-\mathrm{c}_{2} \mathrm{te}^{-\mathrm{j} \theta}}{1-\mathrm{c}_{1} \mathrm{c}_{2} \mathrm{te}^{-\mathrm{j} \theta}}, \mathrm{H}_{\mathrm{drop}}=\frac{\mathrm{s}_{1} \mathrm{~s}_{2} \sqrt{\mathrm{te}^{-\mathrm{j} \theta}}}{1-\mathrm{c}_{1} \mathrm{c}_{2} \mathrm{te}^{-\mathrm{j} \theta}} .
$$

Figure 2.4 shows a plot of the power transmission to the through- and drop-ports of an add-drop ring resonator (solid and dashed line respectively).

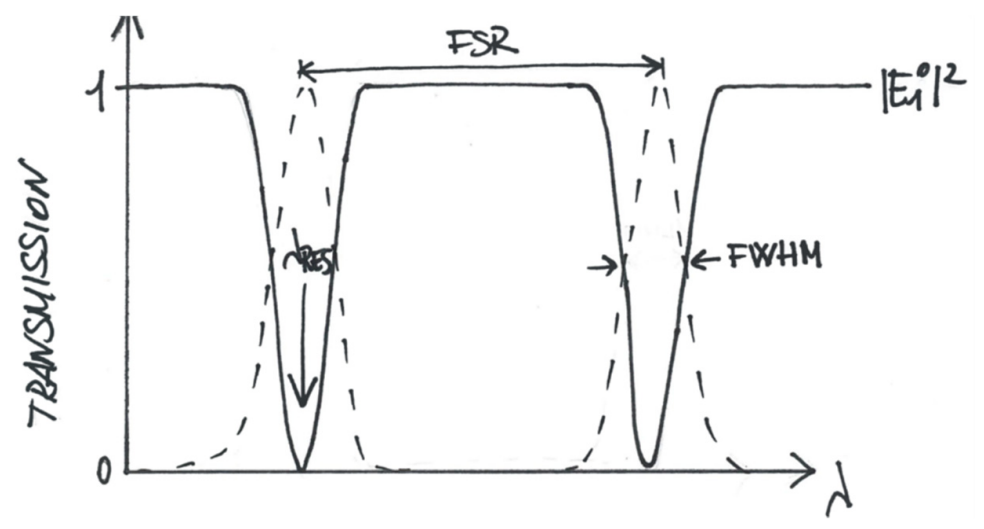

Fig. 2.4 Transmission of an add-drop ring resonator. The solid and dashed lines are $\left|H_{\text {through }}\right|^{2}$ and $\left|H_{\text {drop }}\right|^{2}$, respectively. The resonant wavelengths are the wavelength at which a dip (or peak) occurs. The FSR is the distance between two consecutive resonances.

It can be shown that, neglecting dispersion, the transmission function is a periodic function of the light frequency and thus, approximately, also a periodic function of wavelength as can be seen in Fig. 2.4. The transmission functions can thus essentially be described by two characteristic parameters, namely the free spectral range (FSR) 


\section{Theoretical background}

and the full-width-at-half-maximum (FWHM) of each resonance peak (for drop port output). The FSR, defined as the distance between two consecutive resonances, is given by $\operatorname{FSR}[\mathrm{nm}]=\frac{\lambda_{0}^{2}}{\mathrm{n}_{\mathrm{g}} \mathrm{L}}$ where $\lambda_{0}$ is the vacuum input wavelength, $\mathrm{L}$ is the length of the ring and $n_{g}$ is the group refractive index defined as $n_{g}=n_{e f f}-\lambda \frac{\partial n_{e f f}}{\partial \lambda}$, that can be substituted by the $\mathrm{n}_{\text {eff }}$ if the wavelength dependency of the refractive index can be neglected over one FSR. The FWHM bandwidth of the resonator is related to the quality factor of the resonator, which is a measure of the absolute sharpness of the resonance, i.e., $Q=$ $\frac{\lambda_{\text {res }}}{\mathrm{FWHM}}=\frac{\pi \mathrm{n}_{\mathrm{g}} \mathrm{L}}{\lambda_{\mathrm{o}}} \frac{\sqrt{\mathrm{tc}_{1} c_{2}}}{\left(1-\mathrm{c}_{1} c_{2}\right)}$. A high-Q ring resonator implies high spectral selectivity, which is often desired, e.g., for high-resolution spectral filtering. The given relation between $\mathrm{Q}$ and the resonator building parameters show that, in order to increase the Q-factor of a ring resonator, the cavity length can be increased or the propagation losses need to be reduced, including possible losses inside the directional couplers [14]. Increasing the cavity length is the most straightforward approach in terms of fabrication, however, this also narrows the FSR, i.e., a higher resolution and non-ambiguous transmission wavelength is then available only across a narrower spectral window. A measure of the sharpness of the resonances relative to their spacing is given by the finesse, i.e., $\mathrm{F}=\frac{\mathrm{FSR}}{\mathrm{FWHM}}=\frac{\pi \sqrt{\mathrm{tc}_{1} c_{2}}}{1-\mathrm{c}_{1} c_{2}}$.

\subsubsection{Vernier filter}

Part of the experiments in this thesis have focused on a widening of the spectral window for a high-resolution spectrometer, by extending the free spectral range. A way to extend the FSR without sacrificing spectral selectivity is to couple sequentially

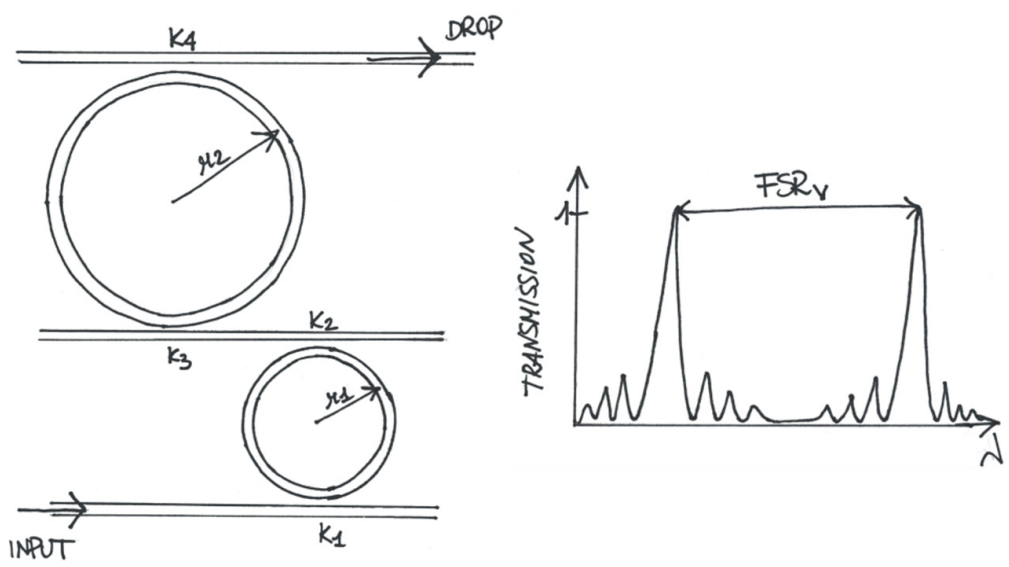

Fig. 2.5 Vernier filter realized with two consecutive add-drop ring resonators of different radii. The drop-port output response of the Vernier filter will show a wider $F S R_{v}$ than the FSRs of the single ring resonators: the light transmitted from the input to the drop port must satisfy simultaneously the resonant conditions of both rings. 
two ring resonators of different radii $r_{1}$ and $r_{2}$ (Fig. 2.5), i.e., in a Vernier configuration. In such setting, light will be transmitted to the drop port of the second RR when incident wavelength, $\lambda_{\text {res, }}$ satisfies the resonant condition for both rings simultaneously, i.e., $m \lambda_{\text {res }}=n_{\text {eff }} L_{1,2}$ with $L_{i}=2 \pi r_{i}$. For a quantitative evaluation, the drop-port transfer function of the Vernier filter $\mathrm{H}_{V}$ can be written as the product of the drop port transfer functions of the two individual add-drop ring resonators,

$$
H_{v}=\left[\frac{s_{1} s_{2} \sqrt{t_{1} e^{-j \theta_{1}}}}{1-c_{1} c_{2} t_{1} e^{-j \theta_{1}}}\right]\left[\frac{s_{3} s_{4} \sqrt{t_{2} e^{-j \theta_{2}}}}{1-c_{3} c_{4} t_{2} e^{-j \theta_{2}}}\right]
$$

The overall FSR of the Vernier filter is given by $\mathrm{FSR}_{\mathrm{v}}=\mathrm{N} \cdot \mathrm{FSR}_{1}=\mathrm{M} \cdot \mathrm{FSR}_{2}$ which implies

$$
\mathrm{FSR}_{\mathrm{v}}=|\mathrm{M}-\mathrm{N}| \frac{\mathrm{FSR}_{1} \cdot \mathrm{FSR}_{2}}{\left|\mathrm{FSR}_{1}-\mathrm{FSR}_{2}\right|}
$$

where $\mathrm{M}$ and $\mathrm{N}$ are integer numbers. If $\mathrm{N}$ and $\mathrm{M}$ are coprime, the FSR of the Vernier filter becomes maximized, i.e., the FSR of the individual ring resonators can be expanded to their least common multiple, limited only by the Q-factor of the individual RR.

Note that the optical response of both a single ring resonator and a Vernier filter can be modified by thermal tuning because of the temperature dependence of $n_{e f f}(T)$, which will thus affect the resonance conditions. Both of these options are explored in Chapter 3 for investigation towards chip-based wavelength meters.

\subsubsection{Mach-Zehnder interferometer}

The second central building block employed in this thesis is the Mach-Zehnder interferometer (MZI), which enables the realization of low-Q spectral filters, wavelength division multiplexers, tunable beam splitters and, with a more complex architecture, programmable photonic processors. An integrated MZI, as shown schematically in Fig. 2.6, has two inputs and two outputs waveguides and consists of
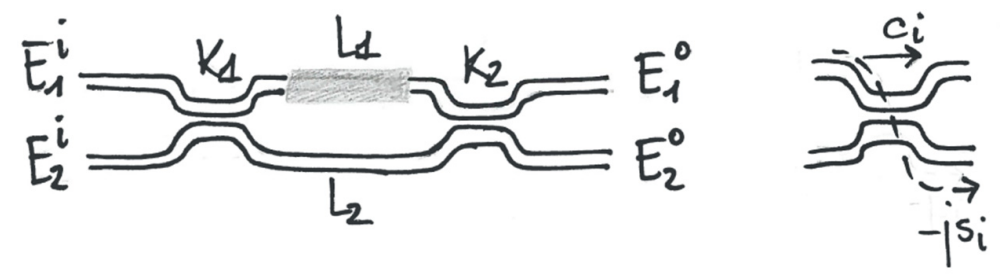

Fig. 2.6 Schematic of an integrated Mach-Zehnder interferometer. The two directional couplers have power coupling ratio $k_{1}$ and $k_{2}$. The field components of each directional coupler are defined on the right. The arms of the interferometer are of length $L_{1}$ and $L_{2}$. 


\section{Theoretical background}

two directional couplers (DC) of power coupling ratio $\mathrm{k}_{1}$ and $\mathrm{k}_{2}$ connected by two waveguides of length $\mathrm{L}_{1}$ and $\mathrm{L}_{2}$, with $\mathrm{L}_{\mathrm{d}}=\left|\mathrm{L}_{1}-\mathrm{L}_{2}\right|$.

The arm length difference $L_{d}$ introduces a phase delay $\theta=\frac{2 \pi n_{\text {eff }}}{\lambda} L_{d}$ between the light travelling in the upper and lower arm of the interferometer, where $\lambda$ is the vacuum wavelength and $n_{\text {eff }}$ is the effective refractive index of the waveguide. The coupling field components of the directional coupler are defined in Fig. 2.6 (right). The transfer matrix of a MZI can be written as the product of the matrices of the two directional couplers and the connecting waveguides that have relative phase difference of $\mathrm{e}^{-\mathrm{j} \theta}$

$$
\begin{aligned}
& \left(\begin{array}{l}
E_{1}^{o} \\
E_{2}^{o}
\end{array}\right)=\left(\begin{array}{cc}
c_{2} & -j s_{2} \\
-j s_{2} & c_{2}
\end{array}\right)\left(\begin{array}{cc}
e^{-j \theta} & 0 \\
0 & 1
\end{array}\right)\left(\begin{array}{cc}
c_{1} & -j s_{1} \\
-j s_{1} & c_{1}
\end{array}\right)\left(\begin{array}{l}
E_{1}^{i} \\
E_{2}^{i}
\end{array}\right) \\
& \left(\begin{array}{l}
E_{1}^{o} \\
E_{2}^{o}
\end{array}\right)=\left(\begin{array}{cc}
c_{1} c_{2} e^{-j \theta}-s_{1} s_{2} & -j s_{1} c_{2} e^{-j \theta}-j c_{1} s_{2} \\
-j s_{2} c_{1} e^{-j \theta}-j c_{2} s_{1} & -s_{1} s_{2} e^{-j \theta}+c_{1} c_{2}
\end{array}\right)\left(\begin{array}{l}
E_{1}^{i} \\
E_{2}^{i}
\end{array}\right)
\end{aligned}
$$

Taking the modulus square of the transfer matrix elements, the transmission power distribution of the interference fringes at the two outputs can be found, as described in Eq. 1.16 and Fig. 2.7 ( $\mathrm{T}_{\text {bar }}$ in yellow and $\mathrm{T}_{\text {cross }}$ in red)

$$
\begin{gathered}
\mathrm{T}_{\text {bar }}=\left|\mathrm{c}_{1} \mathrm{c}_{2} \mathrm{e}^{-\mathrm{j} \theta}-\mathrm{s}_{1} \mathrm{~s}_{2}\right|^{2}=\mathrm{a}_{1}-\mathrm{b}_{1} \cos \theta \\
\mathrm{T}_{\text {cross }}=\left|-\mathrm{js}_{1} \mathrm{c}_{2} \mathrm{e}^{-\mathrm{j} \theta}-\mathrm{j} \mathrm{c}_{1} \mathrm{~s}_{2}\right|^{2}=\mathrm{a}_{2}-\mathrm{b}_{2} \cos (\theta+\pi)
\end{gathered}
$$

where only one non-zero input has been considered for simplicity $\left(E_{2}^{i}=0\right)$ and where $a_{i}$ and $b_{i}$ are the eventual offset and amplitude of the sinusoid. In the following, we will refer to the phase at which light exits the MZI from the same input waveguide, i.e., full reflection, as bar setting (Fig. 2.7 inset top). The phase at which light exits the
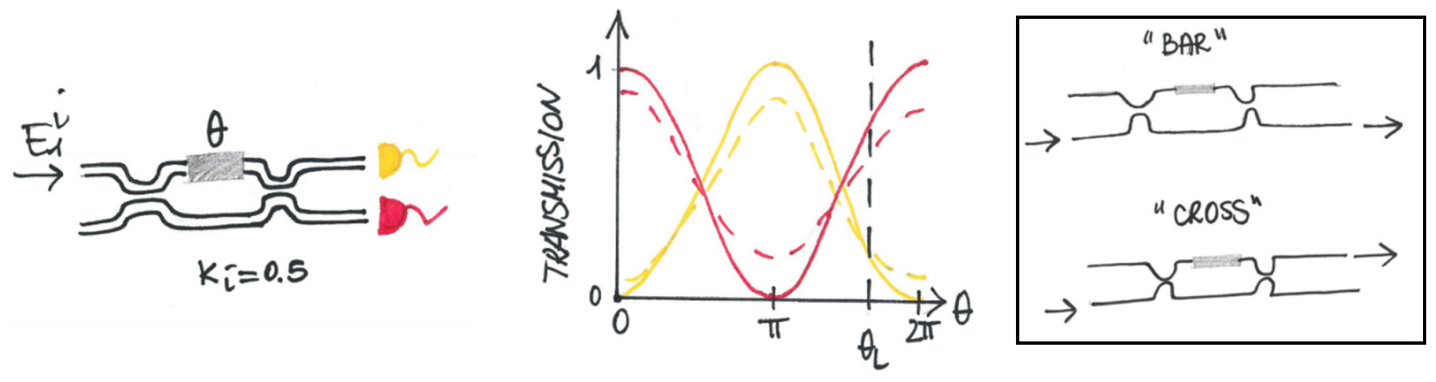

Fig. 2.7 Transmission of a single MZI versus the relative phase delay $\theta$ for balanced directional coupler of $k_{i}=0.5$ considering input light in the top waveguide. If $k_{i} \neq 0.5$ the visibility of the sinusoids reduces (dashed lines). If the thermal tuning element does not provide a full period of phase shift, the sinusoids will run only up to the limiting phase $\theta_{L}$. In the inset is the definition of the bar and cross settings. 
MZI from the other waveguide, i.e., full transmission, will be named cross setting (Fig. 2.7 inset bottom).

It can be seen that the transmission power functions vary as a sine (cosine) function versus the phase delay $\theta$, meaning that modifying the phase delay between the two interferometer arms the power of the transmitted light wave can be adjusted via $\theta$. Thus, tunable beam splitters can be realized with a MZI and a tunable phase delay in one arm of the interferometer. In this thesis, a tunable phase delay is achieved by thermal tuning, as described in section (2.1.1), where $\theta=\frac{2 \pi}{\lambda} \Delta \mathrm{n}_{\text {eff }}$ and $\Delta \mathrm{n}_{\text {eff }}=\mathrm{n}_{\text {eff }}^{1}(\mathrm{~T})-$ $\mathrm{n}_{\text {eff }}^{2}$, i.e., the difference of the effective refractive index of the interferometer arms where only the upper waveguide is heated up to a temperature $\mathrm{T}$.

\subsubsection{An imperfect Mach-Zehnder interferometer}

Of special importance for a photonic processor as presented in Chapter 4 and 5, is that the performance of a MZI depends critically on two factors: the first is the power coupling ratio $\mathrm{k}_{\mathrm{i}}$ of the two directional couplers and the second is the thermal tuned phase delay. Fabrication imperfections might affect the power coupling ratio or induce a phase offset in the interferometer arms, thus changing the transmission function of the MZI. We analyze in this section how imperfections affect the response of a MZI as a theoretical background for explaining the experimental results presented in Chapter 4.

Let's first consider the case of a MZI with imperfect DC of power coupling ratio $\mathrm{k}_{1}$ and $k_{2}$ deviating from their ideal values $k_{i}=0.5, i=1$, 2 . In this case, the visibility of the transmission sinusoidal curve reduces, i.e., full reflection (bar setting) and full transmission (cross setting) are no longer achievable. A 50:50 splitting ratio can usually be still achievable. We define the coupling deviation from the ideal case as $\delta \mathrm{k}_{\mathrm{i}}=\mathrm{k}_{\mathrm{i}}-$ 0.5 . For symmetric (asymmetric) deviation as $\delta \mathrm{k}_{1}=\delta \mathrm{k}_{2}\left(\delta \mathrm{k}_{1}=-\delta \mathrm{k}_{2}\right)$, the bar (cross) setting is always achievable while the full transmission (reflection), i.e., cross (bar) setting, decreases with the square of the coupling deviation $\delta \mathrm{k}_{\mathrm{i}}$ (Fig. 2.8) and thus the visibility of both outputs. We notice that, despite full transmission (reflection) cannot be achieved anymore, the 50:50 splitting ratio can always be achieved for an arbitrary relative phase delay $\theta$, where $\theta=\frac{\pi}{2}$ for the ideal case of $\delta \mathrm{k}_{\mathrm{i}}=0$ (Fig. 2.9). Acquiring the experimental transmission power distribution of the two outputs of each MZI of a processor (Chapter 4) and fitting it with the corresponding transfer functions (Eq. 1.16) we can derive the coupling coefficients $\mathrm{k}_{\mathrm{i}}$ of the directional couplers of each MZI. 


\section{Theoretical background}

Another imperfection that can affect the response of a MZI is the unbalance of the two arms of the interferometer $\left(\mathrm{L}_{1} \sim \mathrm{L}_{2}\right)$. This unbalance induces a fixed relative phase delay $\theta_{\text {offset }}$ between the interfering paths that results in an offset of the interference

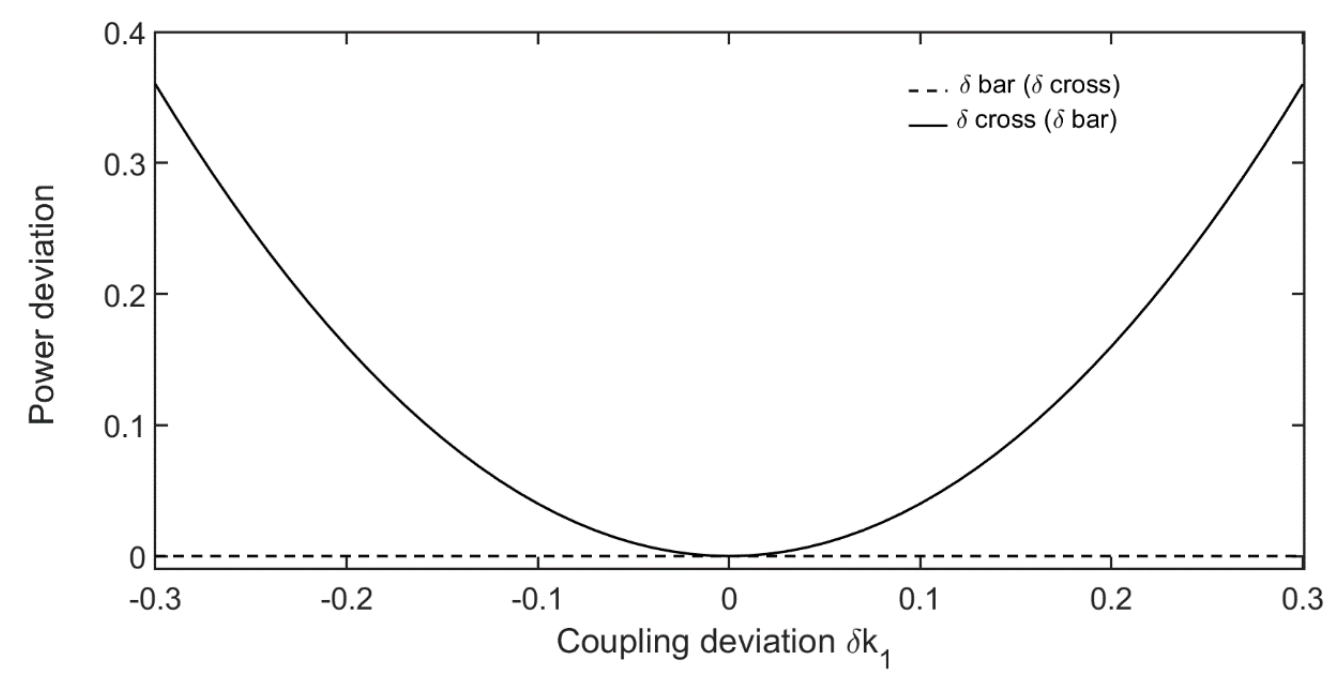

Fig. 2.8 Power deviation of the bar and cross setting from their ideal values, i.e., 1 and zero for symmetric (asymmetric) coupling deviation.

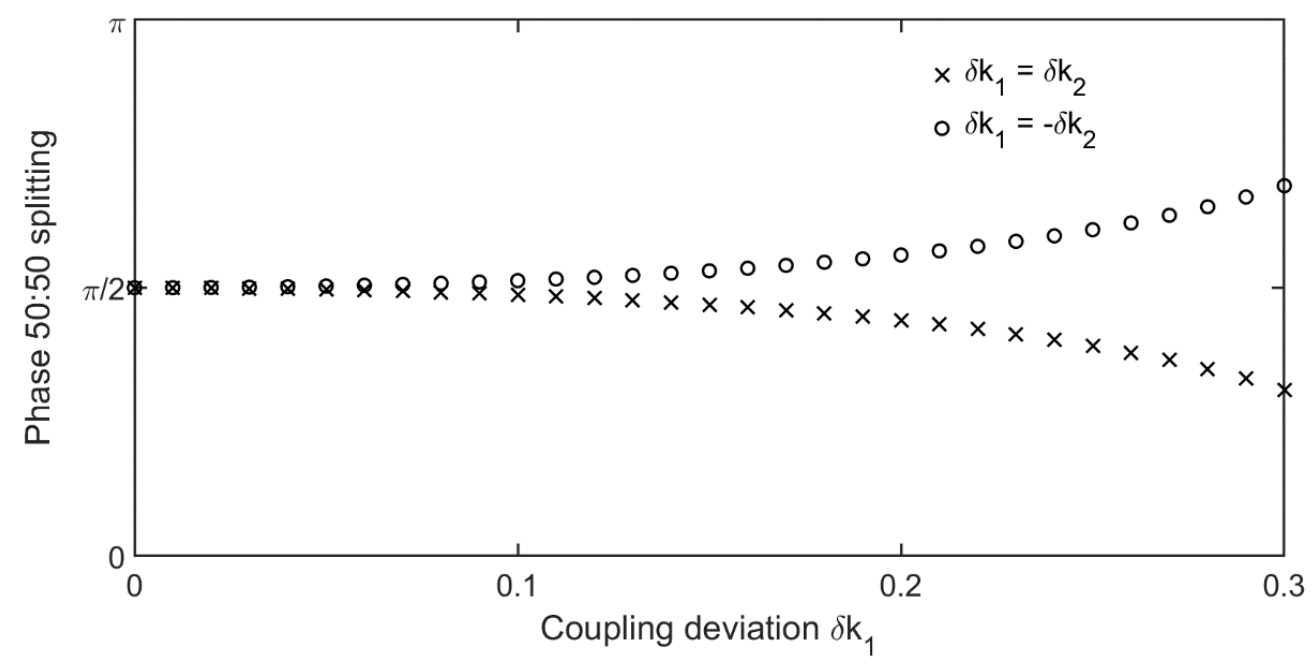

Fig. 2.9 Phase corresponding to a 50:50 power splitting versus the coupling deviation of the first coupler from its ideal value.

fringe of the MZI (Fig. 2.10). The minimum transmission at the lower output mode in fact is no longer at $\theta=0$ but at $\theta_{\text {offset }}=\frac{2 \pi}{\lambda} n_{\text {eff }} L_{\text {offset, }}$ with $L_{\text {offset }}=\left|L_{1}-L_{2}\right| \ll 1$. Knowing the effective refractive index $n_{\text {eff }}$ and the input wavelength we can derive, from the measured phase delay offset $\theta_{\text {offset }}$, the path length offset $L_{\text {offset }}$.

We now consider, ultimately, the imperfection of the thermo-optic phase shifters, i.e., the heater cannot provide a sufficient temperature change dT to induce a $2 \pi$ phase shift. There are two ways of increasing the phase shift induced by a thermo-optic phase 


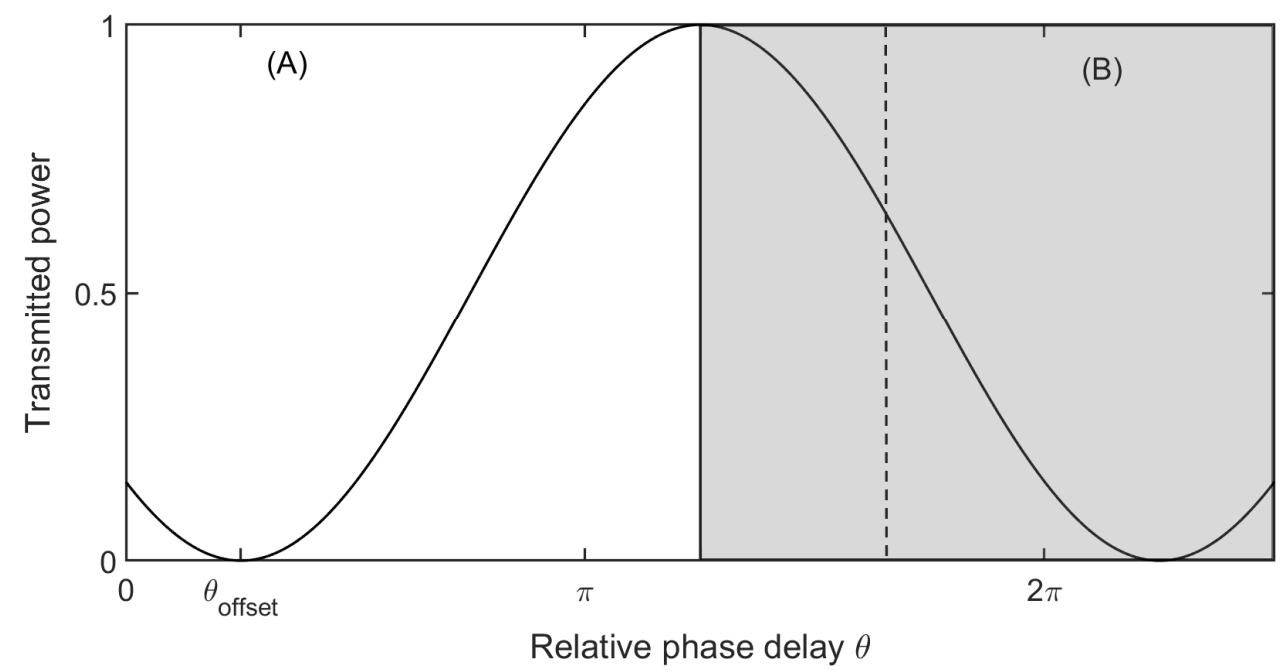

Fig. 2.10 Transmission spectrum of a MZI with unbalanced interferometer arms. The transmission starts with a phase offset proportional to the length difference of the two arms. In case of an imperfect thermo-optic phase shifter, the tuning range is limited (dashed line) preventing the achievement of certain phases.

shifter. We recall (2.1.1) that the phase delay $\theta \propto \mathrm{dT} \cdot \mathrm{L} \propto \mathrm{P}_{\mathrm{e}} \cdot \mathrm{L}=\frac{\mathrm{V}^{2} \mathrm{~L}}{\mathrm{R}}$ where $\mathrm{L}$ is the length of the heater and $\mathrm{P}_{\mathrm{e}}$ is the electrical power generated by the current flowing through a resistive metal heater of resistance $R$ when a voltage $V$ is applied. On one hand, the length $L$ of the heater can be increased. In this way the resistance $R$ increases as well allowing for greater flowing current and thus higher electrical power. On the other hand, we can induce a greater temperature variation dT by directly increasing the electrical power $\mathrm{P}_{\mathrm{e}}$, which means, for a fixed heater length, increasing the applied voltage (paying attention to stay below the damaging threshold). In our case we use chromium-gold thermo-optic phase shifters, giving a typical resistance of $275 \pm$ $25 \frac{\Omega \mathrm{hm}}{\mathrm{mm}}$ with a maximum allowed current $\mathrm{I}_{\max }=40 \mathrm{~mA}$. The typical length to achieve $2 \pi$ phase shift for a SDS waveguide cross-section is $\mathrm{L}=2.2 \mathrm{~mm}$ that corresponds to a maximum voltage of $\sim 28 \mathrm{~V}$.

\subsubsection{Network of Mach-Zehnder interferometers}

Integrated MZIs can be arranged in multi-stage (dashed line in Fig. 2.11) and multichannel (multitude of inputs and outputs) architecture for reconfigurable photonic processors that can be utilized, e.g., for optically assisted machine learning [15] and for quantum information processing applications [16]. For brevity, in the rest of the thesis, we will refer to multi-stage architectures intending both multi-stage and multi-channel networks of MZI. Part of our experiments have used networks of MZI of low intrinsic loss to demonstrate essential functionalities of quantum processing with high fidelity. 


\section{Theoretical background}

For reference to these experiments and data evaluation, we extend our analysis to a network of MZIs that resembles the waveguide circuit that will be presented later in this thesis (Chapter 4 and 5).

Let us consider a network of four reconfigurable unit cells (Fig. 2.11), each defined as a MZI interferometer containing and preceded by a thermal tuning element (grey block).

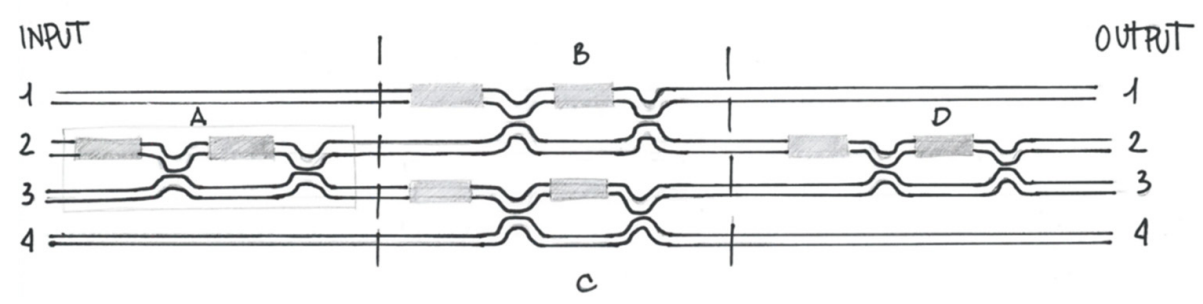

Fig. 2.11 Schematic of a $4 \times 4$ MZI network formed by four unit cells (A, B, C and D) formed each by a phase shifter and a tunable beam splitter.

For brevity and generality, we will refer to the thermal tuning element preceding the MZI as phase shifter (PS) and to the tunable MZI as tunable beam splitter (TBS). For a simplified description, we consider the case where both PS and TBS are ideal, i.e., enable a full 0 -to- $2 \pi$ phase shift and comprise perfectly symmetric couplers, $\mathrm{k}_{1}=$ $\mathrm{k}_{2}=0.5$, and we neglect any losses in the waveguide circuit. The realistic case of a non-ideal couplers and phase shifters has been treated in previous subsection and the experimental results will be presented in Chapter 4 .

Amongst the various methods to determine the response of such a multistage MZIs architecture [17], one is to individually characterize each tunable element of the network.

First, we characterize the tunable beam splitter TBS, (i.e., TBS belonging to unit cell c) as is the element through which light can be directed without having to pass other elements. For its characterization, light is injected in input 4 and the transmitted power versus the tuning phase $\theta_{c}$ is detected at output 4. As described in Section 2.2.3, maximum transmission, i.e., $\mathrm{T}=1$, for the idealized situation considered here, is obtained for $\theta_{c}=\pi$. We will refer to this phase value as the "bar setting". Minimum transmission, i.e., $\mathrm{T}=0$ is obtained for $\theta_{\mathrm{c}}=0$ or $2 \pi$, which is named "cross setting" (Fig. from previous section). For $\theta_{c}=\frac{\pi}{2}$ or $\frac{3}{2} \pi, \mathrm{TBS}_{\mathrm{c}}$ forms a $50 \%$ beam splitter.

Second, $\mathrm{TBS}_{\mathrm{d}}$ in unit cell $\mathrm{d}$ is characterized since light can be injected in it going solely through previously characterized elements. To divert a known amount of light to $\mathrm{TBS}_{\mathrm{d}}$, one can set $\mathrm{TBS}_{\mathrm{c}}$ to cross, knowing that all the light from input 4 exits at the 
top output of $\mathrm{TBS}_{\mathrm{c}}$ and thus enter $\mathrm{TBS}_{\mathrm{d}}$ from its bottom input. The power transmission power of $\mathrm{TBS}_{\mathrm{d}}$ can then be recorded at output 3 varying, this time, $\theta_{\mathrm{d}}$.

Third, for $\mathrm{TBS}_{\mathrm{a}}\left(\mathrm{TBS}_{\mathrm{b}}\right)$ input 3 can be used and the transmission power at output 4 (output 3 ) versus the tuning phase $\theta_{a}\left(\theta_{b}\right)$ can be recorded, given $\operatorname{TBS}_{c}\left(\mathrm{TBS}_{\mathrm{d}}\right.$ and $\left.\mathrm{TBS}_{\mathrm{a}}\right)$ being set to cross.

Fourth, after characterization of all the TBSs, it becomes possible to characterize the inner phase shifters (PSs), i.e., $\mathrm{PS}_{\mathrm{c}}$ and $\mathrm{PS}_{\mathrm{d}}$, by setting the TBSs in a MZI-like configuration, namely, with $\mathrm{TBS}_{\mathrm{a}}$ and $\mathrm{TBS}_{\mathrm{d}}$ to $50 \%$ and $\mathrm{TBS}_{\mathrm{c}}$ and $\mathrm{TBS}_{\mathrm{b}}$ to bar. By injecting light in input 3 , the transmission power versus the phase $\varphi_{c}\left(\varphi_{d}\right)$ can be measured at output 3 , giving access to the relative phase difference of the internal paths of the network. In the ideal case, the sinusoidal transmission curve obtained is similar to the previous ones, where, for $\varphi_{\mathrm{c}}=0$, the transmission power has a minimum. However, if the phases of $\mathrm{TBS}_{\mathrm{a}}$ and $\mathrm{TBS}_{\mathrm{d}}$ differ by $\pi$, i.e., for example $\theta_{\mathrm{a}}=$ $\frac{\pi}{2}$ and $\theta_{d}=\frac{3}{2} \pi$, the response of the MZI-like system is shifted by $\pi$ as well: for perfectly balanced MZI-like arms, i.e., $\varphi_{\mathrm{c}}=0$, the transmission power is maximum. This consideration will be applied in the characterization of an imperfect network in Chapter 4.

With the procedure described above, $\mathrm{PS}_{\mathrm{a}}\left(\mathrm{PS}_{\mathrm{b}}\right)$ remains unknown, however, it can be characterized by using two coherent beams and recombining them properly on the chip. Doing so, the relative phase of the different inputs can be determined. Ideally, the relative phase of the inputs should be zero in order to assure coherent superposition of the signals at each measure, or stage (dashed line on Fig. 2.8) of the network. This means that the optical paths of inputs that encounter the first unit cell on a later stage of the network, e.g., input 1 and 4 , must compensate for the path difference of input that encounter the first unit cell earlier in the network, e.g., input 2 and 3.

\subsection{From classical to quantum linear optical circuits}

Quantum computing and information processing have attracted increasing interest in the last decades because of their promise of solving certain problems and tasks more efficiently or in a more secure way than classically. Examples are the quantum algorithm to factorize integer numbers in their prime factors given by Shor [18], the quantum algorithm for searching in an unstructured search space by Grover [19] or how to build up a shared secret key on a quantum channel in a secure way by Bennett and Brassard [20]. The exciting promises of quantum computation and information 


\section{Theoretical background}

processing originate from the massive parallelism in quantum information processing, inherent in the quantum superposition principle, and in the unclonability of quantum states.

In this thesis we will focus on optical quantum information processing, using single photons as information carriers. This choice is given by the low decoherence, ease of manipulation and light-speed transmission of photons. Linear optical circuits (LOC) can be used as platform for processing quantum information together with single-photon detectors [21]. We will show in Chapter 4 and 5 an integrated LOC for use as quantum photonic processor. We introduce in the following subsections some fundamental concepts connecting the quantum formalism and classical LOCs.

\subsubsection{Quantum linear optical circuits}

In previous sections we introduced the description of linear and lossless optical components as directional coupler, ring resonators and MZIs using the classical formalism for electromagnetic waves. The linear relation between the classical output fields and the classical input fields can be written as a transfer matrix $\mathbf{T}$, i.e., $\mathbf{E}^{\mathbf{o}}=\mathbf{T} \mathbf{E}^{\mathrm{i}}$, where $\mathbf{E}^{\mathbf{o}}$ and $\mathbf{E}^{\mathrm{i}}$ are the output and input column vectors, respectively, containing complex-valued field strength values at the various input and output waveguides of the network, and where $\mathbf{T}$ is symmetric and unitary [22].

Thanks to the second quantization of the electromagnetic field, as, e.g., extensively described by Loudon [23], it is possible to link each energy level of a classical electromagnetic field in a cavity to a quantum harmonic oscillator. This allows expressing a classical electric field in optical mode $\mathrm{j}$ and its complex conjugate, i.e., $\mathrm{E}_{\mathrm{j}}$ and $E_{j}^{*}$, by ladder operators, i.e., respectively the annihilation and creation quantum operator $\mathrm{a}_{\mathrm{j}}$ and $\mathrm{a}_{\mathrm{j}}^{\dagger}$ of a photon of energy $\hbar \omega_{\mathrm{j}}$ in cavity mode $\mathrm{j}$. We are thus able to derive the response of classical optical components in the quantum case, e.g., upon measurements of the transmission of single photons and of quantum correlations between photons. In our case, the optical modes are defined as the waveguide channels where the light travels through.

The quantum operators are related by the same classical transfer matrix $T$, i.e., $b_{i}=$ $\sum_{j=1}^{N} T_{i j} a_{j}$, where $N$, in our case, is the number of optical waveguide paths, i.e., optical modes, and $b_{i}\left(a_{i}\right)$ is the quantum operator associated with the output (input) electric field amplitude $E^{o}\left(E^{i}\right)$. The following rules apply to the operators $b_{i}$ and $a_{i}$

(a) $\mathrm{b}_{\mathrm{i}}=\sum_{\mathrm{j}=1}^{\mathrm{N}} \mathrm{T}_{\mathrm{ij}} \mathrm{a}_{\mathrm{j}} \equiv \mathrm{U}^{\dagger} \mathrm{a}_{\mathrm{i}} \mathrm{U}$
(b) $\mathrm{b}_{\mathrm{i}}^{\dagger}=\sum_{\mathrm{j}=1}^{\mathrm{N}} \mathrm{T}_{\mathrm{ij}}^{*} \mathrm{a}_{\mathrm{j}}^{\dagger} \equiv \mathrm{U}^{\dagger} \mathrm{a}_{\mathrm{i}}^{\dagger} \mathrm{U}$ 


$$
\begin{array}{ll}
a|n\rangle=\sqrt{n}|n-1\rangle & {\left[a_{i}, a_{j}^{\dagger}\right]=\left[b_{i}, b_{j}^{\dagger}\right]=\delta_{i j}} \\
a^{\dagger}|n\rangle=\sqrt{n+1}|n+1\rangle & {\left[a_{i}, a_{j}\right]=\left[b_{i}, b_{j}\right]=0}
\end{array}
$$

where $U$ is a unitary operator that describes the optical system considered and $|n\rangle$ is a Fock state, also known as a photon-number state.

\section{Fock states for multi-channel architectures}

A Fock state $|\mathrm{n}\rangle_{\mathrm{i}}$ is defined as the eigenstate of the Hamiltonian of a quantum harmonic oscillator in mode $\mathrm{i}$

$$
\mathrm{H}_{\mathrm{i}}=\frac{1}{2} \hbar \omega_{\mathrm{i}}\left(\mathrm{a}_{\mathrm{i}} \mathrm{a}_{\mathrm{i}}^{\dagger}+\mathrm{a}_{\mathrm{i}}^{\dagger} \mathrm{a}_{\mathrm{i}}\right)
$$

such that $H_{i}|n\rangle_{i}=\left(n+\frac{1}{2}\right) \hbar \omega_{i}$, where $n$ is the number of photons in the $i$-th mode each having an energy $\hbar \omega_{\mathrm{i}}$. In our case, the Fock state $|\mathrm{n}\rangle_{\mathrm{i}}$ indicates that there are $\mathrm{n}$ photons travelling in the $i$-th optical mode (waveguide channel). For multi-channel architectures each waveguide channel is a different optical mode, e.g., i,j,k..., forming an orthonormal base. A general description of the overall quantum state of a multichannel architecture can be written as a product of the states of the individual modes

$$
\left|\mathrm{n}_{1}\right\rangle_{\mathrm{i}}\left|\mathrm{n}_{2}\right\rangle_{\mathrm{j}}\left|\mathrm{n}_{3}\right\rangle_{\mathrm{k}} \ldots=\left|\mathrm{n}_{1 \mathrm{i}}, \mathrm{n}_{2 \mathrm{j}}, \mathrm{n}_{3 \mathrm{k}}, \ldots\right\rangle,
$$

where $n_{1}, n_{2} \ldots$ are the number of photons in each mode.

\section{Hong-Ou-Mandel effect}

One of the most important quantum phenomena at the base of many quantum information and computation protocols is the Hong-Ou-Mandel (HOM) effect [24], i.e., when two indistinguishable photons meet on a 50:50 beam splitter the probability of simultaneously detecting one photon at each beam splitter output is zero. This is also known as photon bunching effect. We have shown previously that a 50:50 beam splitter can be realized either with a directional coupler of power coupling coefficient $k=0.5$ or with a tunable MZI for $\theta=\frac{\pi}{2}$. We now retrieve the HOM effect on a 50:50 directional coupler by applying the quantum linear optics formalism introduced above. Analogous results are obtained on a tunable MZI for $\theta=\frac{\pi}{2}$.

The expectation value of detecting light at both outputs of a 50:50 directional coupler when injecting $n_{1}$ photons in input 1 and $n_{2}$ photons in input 2 is $\left.\left|\mathrm{b}_{1} \mathrm{~b}_{2}\right| \mathrm{n}_{1} \mathrm{n}_{2}\right\rangle\left.\right|^{2}=\left\langle\mathrm{n}_{1} \mathrm{n}_{2}\left|\mathrm{~b}_{2}^{\dagger} \mathrm{b}_{1}^{\dagger} \mathrm{b}_{1} \mathrm{~b}_{2}\right| \mathrm{n}_{1} \mathrm{n}_{2}\right\rangle$. Given that $\mathrm{b}_{1}=\sum_{\mathrm{j}=1}^{2} \mathrm{~T}_{1 \mathrm{j}} \mathrm{a}_{\mathrm{j}}=\left(\mathrm{ca}_{1}-\mathrm{jsa} \mathrm{a}_{2}\right)$ and $\mathrm{b}_{2}=\sum_{\mathrm{j}=1}^{2} \mathrm{~T}_{2 \mathrm{j}} \mathrm{a}_{\mathrm{j}}=\left(-\mathrm{js} \mathrm{a}_{1}+\mathrm{ca}_{2}\right)$ (Fig. 2.6), the expectation value becomes

$$
\left\langle\mathrm{n}_{1} \mathrm{n}_{2}\left|\left(\mathrm{jsa}_{1}^{\dagger}+\mathrm{ca}_{2}^{\dagger}\right)\left(\mathrm{ca}_{1}^{\dagger}+\mathrm{jsa} \mathrm{a}_{2}^{\dagger}\right)\left(\mathrm{ca}_{1}-\mathrm{jsa} \mathrm{a}_{2}\right)\left(-\mathrm{jsa}_{1}+\mathrm{ca}_{2}\right)\right| \mathrm{n}_{1} \mathrm{n}_{2}\right\rangle
$$




\section{Theoretical background}

that, after applying commutation rules and neglecting terms with odd powers of quantum operators, reduces to

$$
\left\langle n_{1} n_{2}\left|\left(c^{2} s^{2} n_{1}^{2}+s^{4} n_{1} n_{2}-c^{2} s^{2} n_{1}\left(n_{2}+1\right)-c^{2} s^{2} n_{2}\left(n_{1}+1\right)+c^{4} n_{1} n_{2}+c^{2} s^{2} n_{2}^{2}\right)\right| n_{1} n_{2}\right\rangle \text {. }
$$

If $\mathrm{n}_{1}=\mathrm{n}_{2}=1$ the expectation value of detecting a photon at each exit of the directional coupler becomes

$$
\left(-2 c^{2} s^{2}+c^{4}+s^{4}\right)\langle 11 \mid 11\rangle=\left(c^{2}-s^{2}\right)^{2} .
$$

The term $\left(-2 c^{2} s^{2}\right)$ corresponds to the event of one photon (from input 1) being reflected with power coefficient $c^{2}$ and one photon (from input 2) being transmitted with power coefficient $-s^{2}$, and vice versa (factor 2). The fourth-power terms indicate the event of both photons being reflected $\left(c^{4}\right)$ or transmitted $\left(s^{4}\right)$. If the directional coupler has power coupling ratio of $\mathrm{k}=0.5$ then $\mathrm{c}=\mathrm{s}=\sqrt{0.5}$ leading to

$$
\left(\sqrt{0.5}^{2}-\sqrt{0.5}^{2}\right)^{2}\langle 11 \mid 11\rangle=0
$$

which indicates that there are no expected photons at each output, i.e., we find back the HOM effect or photon bunching [24].

The probability of photon bunching can be determined by the evolution of the input state, i.e.,

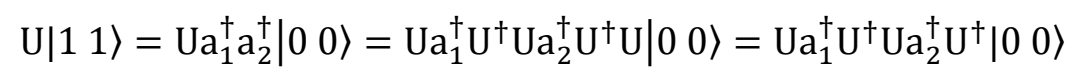

where $U$ is a unitary operator describing the direction coupler and where, in the last equality, we have used that a beam splitter has no effect on the vacuum state, i.e.,

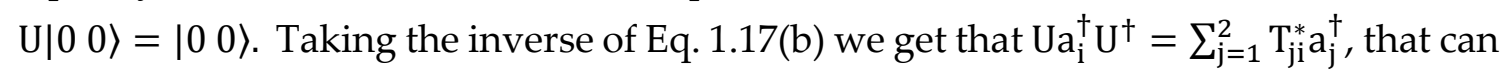
be inserted into Eq. 1.19, giving

$$
\left(\mathrm{ca}_{1}^{\dagger}-\mathrm{jsa}_{2}^{\dagger}\right)\left(-\mathrm{jsa}_{1}^{\dagger}+\mathrm{ca}_{2}^{\dagger}\right)|00\rangle=-\mathrm{j} \sqrt{2} \mathrm{cs}(|20\rangle+|02\rangle)+\left(\mathrm{c}^{2}-\mathrm{s}^{2}\right) \mid 1
$$

For the case of a 50:50 directional coupler, i.e., $c=s=\sqrt{0.5}$, we find thus that the

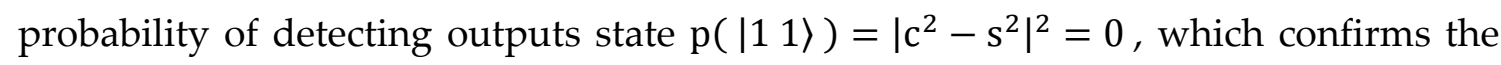
result obtained before. The probability of photon bunching should thus sum up to 1 , as it's the only event allowed, and in fact is given by

$$
\begin{aligned}
& p\left(\mid \begin{array}{ll}
2 & 0\rangle
\end{array}\right)=|-j \sqrt{2} \mathrm{cs}|^{2}=\frac{1}{2}
\end{aligned}
$$

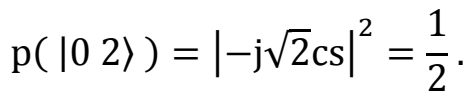

In Chapter 5 we report the measurements of many HOM effects on an integrated MZI multi-channel architecture. 


\section{Single qubit quantum logic gates}

Quantum logic gates are the basic elements to manipulate quantum information. In optical quantum information processing, single photons are the preferred information carriers that transport information in one of their degree of freedom, e.g., polarization, orbital angular momentum or arrival time. In linear optical circuits, a convenient degree of freedom for information encoding is the optical paths or mode, i.e., waveguide channel, that the single photons travel through. We can thus define the quantum information bit or qubit, i.e., the quantum analog of the classical information bit (see inset), in a dual-rail encoding, e.g., a two-channel waveguide optical element such as a MZI (Fig. 2.12).

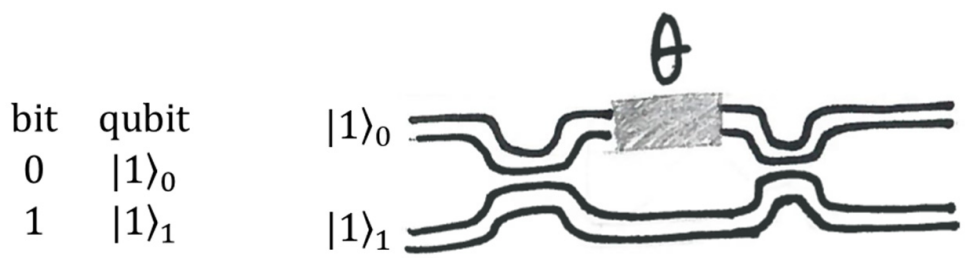

Fig. 2.12 Dual-rail qubit representation. $|1\rangle_{i}$ indicates a photon-number state of 1 photon travelling in mode i that, in this case, correspond to information 0 or 1.

For example, a quantum analog of a logic NOT gate that inverts the bits, i.e., $0 \rightarrow$ 1 and $1 \rightarrow 0$, can be realized by a tunable MZI on cross setting, i.e., $\theta=0$.

Higher information content can be achieved by increasing the dimensionality of the encoding, i.e., adding optical paths. For multistage architectures the information content can be extended as much as the input channels are. In Chapter 5 we show an 8-dimensional rail encoding realizing an 8-dimensional X-gate, i.e., NOT gate.

\section{Fidelity}

An important parameter for quantum information processing is the fidelity $\mathcal{F}$ of a process. The fidelity can be defined also for classical information as the distance measure between two probability distributions $\mathrm{p}_{\mathrm{x}}$ and $\mathrm{q}_{\mathrm{x}}$ as $\mathcal{F}\left(\mathrm{p}_{\mathrm{x}}, \mathrm{q}_{\mathrm{x}}\right)=\sum_{\mathrm{x}} \sqrt{\mathrm{p}_{\mathrm{x}} \cdot \mathrm{q}_{\mathrm{x}}}$ [25]. The fidelity is a dimensionless quantity. In fact, for identical distributions $\mathcal{F}\left(\mathrm{p}_{\mathrm{x}}, \mathrm{p}_{\mathrm{x}}\right)=\sum_{\mathrm{x}} \mathrm{p}_{\mathrm{x}}=1$.

The fidelity can be used as a measure of the similarity of two transformations, e.g., a target $U$ and a constrained $\widetilde{U}$ realized on a multi-stage architecture as described in

Chapter 4, when considering their module squared, i.e., $|U|^{2}$ and $|\widetilde{U}|^{2}$. The power distribution associated with an arbitrary unitary transformation $\mathrm{U}$ can be considered 


\section{Theoretical background}

as a probability distribution, as the powers over each column or row of the matrix are normalized to 1 . In Chapter 4 and 5 we calculate, e.g.,

the fidelity between the probability distribution associated with a set of target unitaries $\left|\mathrm{U}^{\mathrm{i}}\right|^{2}$ and their experimental realizations $\left|\mathrm{U}_{\mathrm{exp}}^{\mathrm{i}}\right|^{2}$.

\subsection{Neural Networks}

In Chapter 3 we present a wavelength meter based on a single ring resonator and a socalled smart readout. The smart readout combines a neural network (NN) and an optimization algorithm to investigate the options towards a temperature-driftimmune wavelength meter. Such a smart readout will be described in Chapter 3 in more detail. Here we briefly recall, for later reference, the basic working principle of a neural network as it is implemented as standard algorithm in mathematical software packages (Matlab).

The basic components of a neural network are neurons that can be stacked in layers, each of which receives an input signal and provides an individual output signal (Fig. 2.13). The output from each neuron is weighted $\left(\mathrm{w}^{\mathrm{j}}\right)$ and the sum of their output signals is sent to each neuron of the next layer. The throughput of the last-layer neurons is the weighted sum signal of all previous neurons.

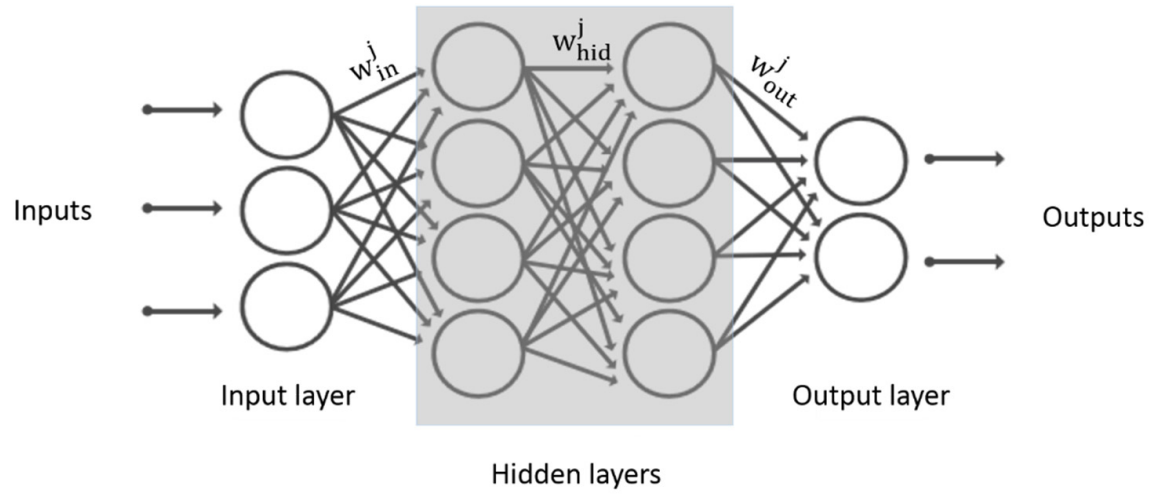

Fig. 2.13 Schematic of a Neural Network. The output signal of each neuron (circle) is weighted by a certain coefficient $\left(w^{j}\right)$.

To represent also nonlinear relations between input and output signals, the inputoutput response of a single neuron is a highly nonlinear function. For instance a typical neuron input-output response function resembles a step-shaped increase of the output vs input. The shape of the step is typically defined with only a few parameters, for instance four parameters. A widely used type of step function in neural networks, which is also used in this thesis, are sigmoid functions, 


$$
f(x)=\frac{c}{1+e^{a(x-b)}}+d
$$

In this expression, $\mathrm{a}, \mathrm{b}, \mathrm{c}$, and $\mathrm{d}$ are fit parameters that describe the slope of the step function (c) to scale the weight of the neuron response, the bias for activation (b) describing at which input value $x$ the step occurs, while $d$ and a define the upper and lower plateaus of response at low and high input value $x$, respectively.

NNs are very attractive tools for mimicking the behavior of complex systems lacking an analytical and exact description, and for which instead only an input-output relation is known, as with the non-understood internal response of a "black box". A NN will give a reasonable approximation of the response of such black box, if the NN is trained using a sufficiently large set of input-output data from the black box. Training the NN is just another term for setting (e.g. via a least-square fit) all the fit parameters $(a, b, c, d)$ of all neurons such that the NN generates a response that maximally closely resembles the response of the black box.

The advantage of a NN compared to physically modelling the response of a complex system, which requires detailed knowledge of the internal processes within the system, is that NN are able to "learn" from the training data set. "Learning" means that the NN generalizes the behavior of the training data set via interpolation and extrapolation, requiring fewer fit parameters than an analytical model. In our case, we use a readily programmed NN (Matlab toolbox), based on two layers of neurons, to mimic the wavelength dependent transmission function of a waveguide ring resonator measured at a larger number of different temperature settings for the waveguide heater. To give an impression on the capability of the NN of generalizing and thus being more efficient in the number of fit parameters compared to analytical methods, we report the case of two resonators in Vernier configuration as presented in Chapter 3: each Vernier transmission spectrum contains approximatively 30 measured peaks corresponding to a maximum of 37 peaks in the prospected model range. The maximum total number of fit parameters required is 37 (peaks) $\times 49$ (thermal tuning settings $) \times 8$ (fit parameters per peak $)=14504$. To provide an accurate modelling of the response of this system, we used a two-layer NN of 200 neurons, involving thus 200 weights and 200 biases. The training of the NN on the measured transmission data thus involves finding 200 (weights) +200 (biases) +200 (weights) $\times 49$ (thermal tuning settings) $=10200$ fit parameters. 


\section{References}

[1] Bauters, J.F., et al., “Ultra-low-loss high-aspect-ratio Si3N4 waveguides”. Optics Express 19(4), p. 3163-3174 (2011).

[2] Roeloffzen, C.G.H., et al., "Low-Loss Si3N4 TriPleX Optical Waveguides: Technology and Applications Overview". IEEE Journal of Selected Topics in Quantum Electronics 24(4), p. 1-21 (2018).

[3] Marcatili, E.A.J., “Dielectric rectangular waveguide and directional coupler for integrated optics". The Bell System Technical Journal 48(7), p. 2071-2102 (1969).

[4] Hocker, G.B. and W.K. Burns, "Mode dispersion in diffused channel waveguides by the effective index method". Applied Optics 16(1), p. 113-118 (1977).

[5] Bahaa E. A. Saleh, M.C.T., Fundamentals of Photonics, John Wiley \& Sons, Inc. (1991).

[6] Arbabi, A. and L.L. Goddard, "Measurements of the refractive indices and thermo-optic coefficients of Si3N4 and SiOx using microring resonances". Optics Letters 38(19), p. 3878-3881 (2013).

[7] Marpaung, D., J. Yao, and J. Capmany, "Integrated microwave photonics". Nature Photonics 13(2), p. 80-90 (2019).

[8] Nitkowski, A., A. Baeumner, and M. Lipson, "On-chip spectrophotometry for bioanalysis using microring resonators". Biomedical Optics Express 2(2), p. 271277 (2011).

[9] Zheng, S.N., L.K. Chin, and A.Q. Liu. "On-chip Spectrometer Enhanced by Ring Resonator Cavity: High-resolution and Large-bandwidth". in Conference on Lasers and Electro-Optics. San Jose, California (Optical Society of America 2017), paper JTh2A.119.

[10] Yebo, N.A., et al., “On-Chip Arrayed Waveguide Grating Interrogated Siliconon-Insulator Microring Resonator-Based Gas Sensor". IEEE Photonics Technology Letters 23(20), p. 1505-1507 (2011).

[11] Okawachi, Y., et al., "Octave-spanning frequency comb generation in a silicon nitride chip". Optics Letters, 36(17) p. 3398-3400 (2011).

[12] Demirtzioglou, I., et al., "Frequency comb generation in a silicon ring resonator modulator". Optics Express, 26(2) p. 790-796 (2018).

[13] Christi K. Madsen, J.H.Z., Optical Filter Design and Analysis, A Signal Processing Approach. Jon Wiley \& Sons, Inc. (1999). 
[14] Spencer, D.T., et al., "Integrated waveguide coupled Si3N4 resonators in the ultrahigh-Q regime". Optica, 1(3) p. 153-157 (2014).

[15] Shen, Y., et al., "Deep learning with coherent nanophotonic circuits". Nature Photonics 11, p. 441 (2017).

[16] Carolan, J., et al., “Universal linear optics”. Science 349(6249), p. 711 (2015).

[17] Miller, D.A.B., "Self-aligning universal beam coupler". Optics Express 21(5), p. 6360-6370 (2013).

[18] Shor, P.W. "Algorithms for quantum computation: discrete logarithms and factoring". in Proceedings 35th Annual Symposium on Foundations of Computer Science (1994).

[19] K. Grover, L., "Fast quantum mechanical algorithm for database search". In Proceedings of the 28th annual ACM symposium on Theory of Computing (1996).

[20] Bennett, C. and G. Brassard, "Quantum cryptography: Public key distribution and coin tossing". Theoretical computer science 560, p. 175-179 (1984).

[21] Knill, E., R. Laflamme, and G.J. Milburn, "A scheme for efficient quantum computation with linear optics". Nature 409(6816), p. 46-52 (2001).

[22] Skaar, J., J.C. García Escartín, and H. Landro, “Quantum mechanical description of linear optics". American Journal of Physics 72(11), p. 1385-1391 (2004).

[23] Loudon, R., The Quantum Theory of Light, Oxford University Press (1973).

[24] Hong, C.K., Z.Y. Ou, and L. Mandel, "Measurement of subpicosecond time intervals between two photons by interference". Physical Review Letters 59(18), p. 2044-2046 (1987).

[25] Nielsen, M.A. and I.L. Chuang, Quantum Computation and Quantum Information, Cambridge University Press, 10th Anniversary Edition (2011). 
44 I P a g e 


\section{Smart wavelength meter based on ring resonators}

\subsection{Towards a temperature-drift-immune wavelength meter based on a single ring resonator}

We present an integrated optical wavelength meter based on a $\mathrm{Si}_{3} \mathrm{~N}_{4} / \mathrm{SiO}_{2}$ microring resonator (operating over a free spectral range of $\approx 2.6 \mathrm{~nm}$ ). The wavelength meter readout is performed by a neural network and an optimization algorithm. This novel approach ensures a high wavelength estimation precision of $\approx 50 \mathrm{pm}$, corresponding to a fractional wavelength stability of $\sim 10^{-5}$. We observe a long-term reproducibility of the wavelength meter response over a time interval of one week. When investigating the influence of deliberately applied changes of the ambient temperature on the estimated wavelength, we observe an immunity of the displayed output wavelength to temperature changes within a range of several degrees ${ }^{\circ} \mathrm{C}$, although the reasons for this effect will need further clarification.

\subsubsection{Introduction}

Optical sensing devices are important for many applications such as in fingerprint spectroscopy [1], in telecommunication systems [2] and in wavelength metrology [3]. A high spectral resolution is a desirable property of optical sensing devices for, e.g., spectroscopic applications [1, 3]. However, state of the art high-resolution spectrometers are typically rather big and expensive devices with critical optomechanical stability. In comparison, waveguide integration of high-resolution spectrometers would offer several advantages, e.g., reduced size and increased longterm stability. Within the field of integrated optical sensing, devices such as microring resonators (MRRs) are increasingly being investigated [4-6]. The reason is that a high spectral resolution can be achieved by high-Q-factor MRRs, limited only by propagation losses. For instance, MRRs have been fabricated in $\mathrm{Si}_{3} \mathrm{~N}_{4}$ technology achieving extremely high Q-values in the order of 80 million [7]. A second feature of MRRs in $\mathrm{Si}_{3} \mathrm{~N}_{4}$ technology is that the resonant frequency can be tuned over a Free Spectral Range (FSR) straightforwardly, particularly when using thermal tuning [8]. Tunable, high-Q MRRs are therefore attractive as on-chip building blocks, to be 
integrated for instance together with light sources as a high-resolution wavelength meter [9], to monitor their wavelength stability or tuning. However, for such applications the reproducibility of the wavelength meter output response is of central importance, particularly in the presence of external perturbations, e.g., temperature changes.

In the case of using a MRR as wavelength meter, a thermal drift would affect the spectral output by shifting its resonant frequencies. To improve reproducibility, different methods may be applied, e.g., thermal isolation and stabilization; repeated calibration with an additional reference light source of well-known and stable wavelength; or using an athermal design of the optical structure involving negative thermo-optic materials [10].

Here we present an integrated optical wavelength meter based on a single MRR and investigate its longer term reproducibility. Within a certain temperature range of a few degrees we find that the displayed wavelength does not change vs deliberately varying the ambient temperature, without relying on thermal stabilization, repeated calibration with additional light sources or an athermal design. Our investigations make use of a MRR based on $\mathrm{Si}_{3} \mathrm{~N}_{4}$ waveguides, motivated by the option of low waveguide loss and the particular maturity of this waveguide platform [11]. The transmission of the MRR is analyzed with a neural network (NN) and an optimization algorithm for wavelength estimation, a so-called smart readout.

We implement the smart readout method in an experimental setup comprising a tunable laser coupled into a MRR and a detector that measures the transmitted power. Based on transmission measurements, the readout determines the wavelength of the input light. We demonstrate, for the first time, the full operation of such a wavelength meter including a long-term reproducibility test over one week and beyond. The investigated wavelength meter is operational across one free spectral range (FSR) of the $\operatorname{MRR}(\approx 2.6 \mathrm{~nm}$ ) with a spectral resolution as high as $\approx 50 \mathrm{pm}$. An extension of the FSR appears possible using an additional resonator as described in section 1.2.

\subsubsection{Principle of data readout algorithm}

In order to explain the basic principle of our data readout algorithm, let us consider the measurement of an unknown wavelength of light injected into an arbitrary wavelength meter. The precision and reproducibility of the displayed wavelength are the central features of a wavelength meter, but the measurement can only be trusted if certain conditions are fulfilled. For instance, the wavelength meter must have a valid calibration and it must operate in the specified environmental parameter range, and 
the validation of these conditions requires more than a single measurement. With many measurements it is possible to determine or improve the calibration of the wavelength meter, and then use further measurements for obtaining knowledge also about an unknown injected wavelength. For instance when $n$ measurements are performed with the same input wavelength this knowledge is a distribution of values for the displayed wavelength.

To obtain such a distribution, one can perform the $n$ measurements of the input light also on $n$ different wavelength meters that can be realized, in our case, with $n$ MRRs of different radii, thus, providing different resonant conditions. Three statistical properties can be obtained from such a distribution. The first is the most likely value for the input wavelength, which is obtained as the mean value of the distribution. This value would only be influenced by a common drift of all devices. The second property is the overall precision obtained by the ensemble of independent devices, which can be specified for instance via the root-mean-square (rms) deviation from the mean value. The precision is expected to improve with the number of devices or measurements, $n$, assuming that the changes of the spectral response of the wavelength meters are uncorrelated for instance, that there is no common drift. The third property is the wavelength deviation of the response of some of the devices as compared to the distribution of the majority of devices: the observation of so-called outliers. The presence of outliers indicates that the corresponding devices have probably lost their calibration. This information can then be used to recalibrate these devices with the mean value of the other devices, in order to improve the overall precision of the estimation.

We note that properly identifying outliers for recalibrating the according devices is not straightforward, because it requires quantifying the importance of each individual measurement in relation to all the other ones. An according "smart" algorithm would have to judge with what statistical weight each individual measurement has to be interpreted as an outlier, and then decide whether and how much to recalibrate (i.e. re-interpret) the response of the corresponding device.

An issue related to such re-calibration can be that, ultimately, during the course of longer measurement series, all of the devices may be identified as outliers and become re-calibrated. This might lead to a wrong wavelength display of all devices, especially if the drift of devices is correlated by some common external perturbation. An example would be deliberately exposing most of the devices to a change of the ambient temperature, in order to induce a common thermal drift in their response. In this case a small number of non-drifting devices, although protected, e.g., by passive thermal 
shielding or active stabilization, would nevertheless be "re-calibrated" to display a wrong wavelength as well.

The wavelength meter we present here provides a wavelength dependence of its response via an optically integrated waveguide MRR on a chip. Sending the transmitted power to a photodetector generates a wavelength-dependent electrical response signal in the form of a Fabry-Perot resonator transmission spectrum. Important is that this wavelength dependence of the response (i.e., the spectral location of transmission peaks) can be changed by tuning the optical length of the resonator to a number of different predetermined and reproducible values. In our experiments we use controlled heating of the waveguide resonator length, to set it sequentially to $n$ different values and thereby effectively realize measuring a given input wavelength with $n$ different response functions. This mimics measuring the same input wavelength with $n$ physically different MRR devices.

To obtain an initial set of data for a calibration, we record for each of the $n$ resonator lengths the response for a large number of known wavelengths across a range that is wider than the free spectral range of the resonator. To prepare the MRR as a wavelength meter with the calibration data, we use a multidimensional function in the form of a NN and fit it to the calibration data that were obtained with the $n$ mimicked wavelength meters. The fit yields a large number of neural network weight factors as global fit parameters that approximately represent all information contained in the much bigger set of measured calibration data.

Finally, when using the wavelength meter for determining the value of an unknown input wavelength, $\lambda_{\mathrm{u}}$, we perform $n$ measurements of the electric response signal, $\mathrm{S}_{\mathrm{u}}^{n}$, by setting the resonator length to the same $n$ values as used for the calibration. Then an algorithm inserts into the neural network fit function a variety of different wavelengths, searching for a wavelength, $\lambda_{\mathrm{e}}$, that would generate $n$ signals, $\mathrm{S}_{\mathrm{e}}^{n}$, with a minimum (least-square) deviation from the $n$ measured values, $\mathrm{S}_{\mathrm{m}}^{n}$. The found or estimated wavelength, $\lambda_{\mathrm{e}}$, which minimizes the least-square deviation, is displayed as the determined input wavelength, also called best wavelength estimate for the unknown input.

A first advantage of such a smart readout is that it is not limited to a monotonously increasing or decreasing output response, which would be required with a standard look-up table approach, for instance using the calibration curve of a single wavelength meter. Thereby a smart readout may widen the spectral range across which unknown wavelengths can be measured. A second advantage is that the smart readout does not rely on any specific physical property of the used optical system. As long as there is a 
wavelength dependent and reproducible response, even if the response is physically not fully understood or too complex for physical modeling, the smart readout can be applied.

There is a strong practical advantage of such a black-box approach that puts the focus only on the optical input-output relation. The optical transmission spectrum of the device does not have to be carefully optimized during fabrication and may even vary strongly from device to device. This advantage is actually what enables using integrated optical devices, as we show here, where a nanoscale precision in fabrication cannot always be reached and where residual reflections at interfaces, such as with input and output fibers, cannot easily be removed. This is quite different with classical wavelength meters and spectrometers where a high precision and reproducibility in fabrication must be guaranteed.

The last advantage is that a NN may be able to generalize the response of the system at which it is trained, i.e., it interpolates using the calibration data set. Thereby a NN is able to predict the response of the system also for unknown configurations, in our case, unknown input wavelengths, via the analytical response function obtained by the NN fit, without the need of physically modelling the system.

\subsubsection{Experimental setup and results}

Figure 3.1 shows the experimental setup (left) and the design of the waveguide resonator chip (right). As the light source we use a single-frequency external cavity diode laser (Santec, TSL-210) that is tunable from $1530 \mathrm{~nm}$ to $1610 \mathrm{~nm}$ and delivers an
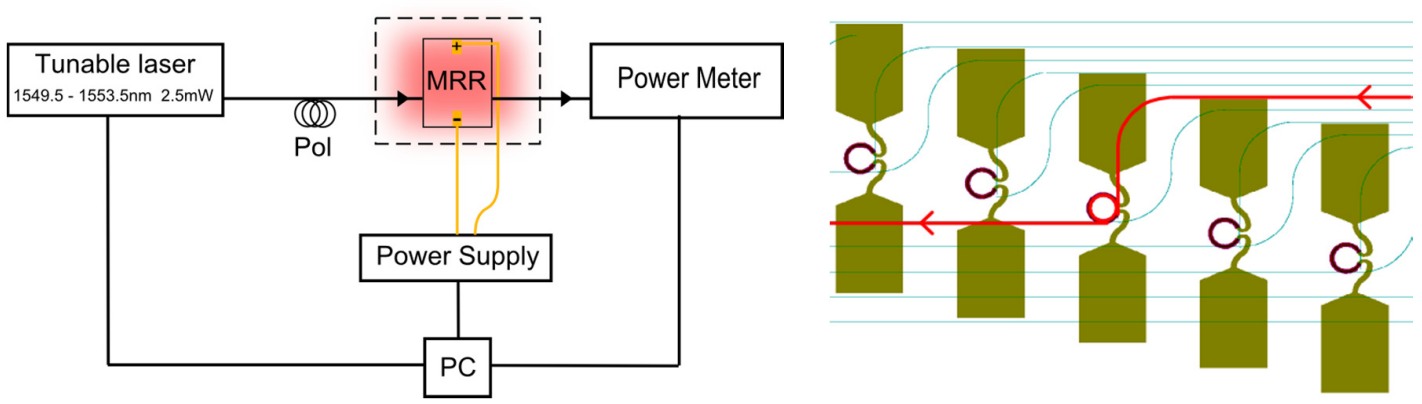

Fig. 3.1 (left) Experimental setup. The tunable laser, power meter and the power supply for the thin film resistive waveguide heaters are controlled by a PC. The light from the laser is coupled into the MRR through a lensed fiber. A twisted polarizer ( $\mathrm{Pol}$ ) controls the state of polarization. A metallic box (dashed frame) equipped with a heating resistor is built around the chip to allow the increasing of the ambient temperature in the box (red shadow). (right) Mask design of the chip under investigation. For the experiments, the third MRR (waveguide shown in red) has been chosen as illustrated. The electrode pads and electric microwires (shown in green) enable the sending of an electric current through thin film heaters located on top of the waveguide resonator, for tuning the optical roundtrip path length of the MRR via thermally induced changes of the refractive index. 
output of $2.5 \mathrm{~mW}$ from a single-mode fiber. The generated laser wavelength is available as digital output from the laser module, with an accuracy of $0.1 \mathrm{~nm}$, a resolution and stability of $0.01 \mathrm{~nm}$, and a repeatability of $0.05 \mathrm{~nm}$, according to manufacturer's specification. A twisted fiber polarizer is used to set the polarization to linear (quasi-TE, for which the MRR is designed). The laser light is coupled via a lensed fiber into one of the input waveguides of the chip. The MRR is fabricated from high-contrast $\mathrm{Si}_{3} \mathrm{~N}_{4}$ waveguides with a box-shaped core cross section embedded in a $\mathrm{SiO}_{2}$ cladding (see Chapter 2) which provides tight guiding and enables short resonator lengths [12], as to provide a wider free spectral range (FSR) of operation. Due to the slightly asymmetric cross section, the two orthogonal polarization modes, quasiTE and quasi-TM, have slightly different propagation constants. The radius of the resonator is $85.47 \mu \mathrm{m}$, which provides a FSR of about $2.6 \mathrm{~nm}(\approx 323 \mathrm{GHz})$ at around $1550 \mathrm{~nm}$ for the TE polarization $(\approx 322 \mathrm{GHz}$ for TM). The input and output power coupling coefficients are designed to be in the order of 0.26 , yielding an expected quality factor of $Q \approx 4000$ ( $\approx 400$ pm FWHM spectral bandwidth). In order to thermally tune the optical length of the resonators to a set of different values, a heating current can be sent through lithographically fabricated, thin-film resistor wires (chromium, with low-resistance gold leads and electrode pads). Typically, applying a potential difference of $10 \mathrm{~V}$ changes the resonator length by one FSR within 1ms. Light transmitted through the resonator and exiting the output waveguide is collected via a second lensed fiber, and the optical power is recorded with a fiber-coupled photodiode (Thorlabs, S154C). To record data over long time intervals, the laser wavelength tuning and heater voltage settings are remotely controlled, while the input laser wavelength (received from the laser module) and the photodiode output signals are recorded, using a computer with standard software (Matlab).

First, the wavelength meter is calibrated. Starting with zero heater voltage, the input wavelength is swept from $1549.5 \mathrm{~nm}$ up to $1553.5 \mathrm{~nm}$, in steps of $0.01 \mathrm{~nm}$. For each input wavelength the transmitted output power is averaged over a time interval of $300 \mathrm{~ms}$, followed by averaging over 50 measurements. This measurement series is repeated for stepwise increased heater voltage, from $0 \mathrm{~V}$ to $10 \mathrm{~V}$ in steps of $0.5 \mathrm{~V}$ (accuracy of setting $\approx 10 \mathrm{mV}$ ). In total the calibration data comprise 8421 photodiode signal values for the MRR output power, obtained with 401 input different wavelengths and 21 different heating voltages. 
The calibration data are fitted by a two-layer neural network (NN) function with 41 neurons and a total of 943 fit parameters (MathWorks, Matlab) that minimize the rms deviation from the calibration data. Figure 3.2 shows a comparison of the obtained NN calibration function and the calibration data, displayed vs wavelength for the 21 heating voltages. It can be seen that, for each wavelength value inserted, the NN function predicts 21 power values, which lie close to the calibration data.

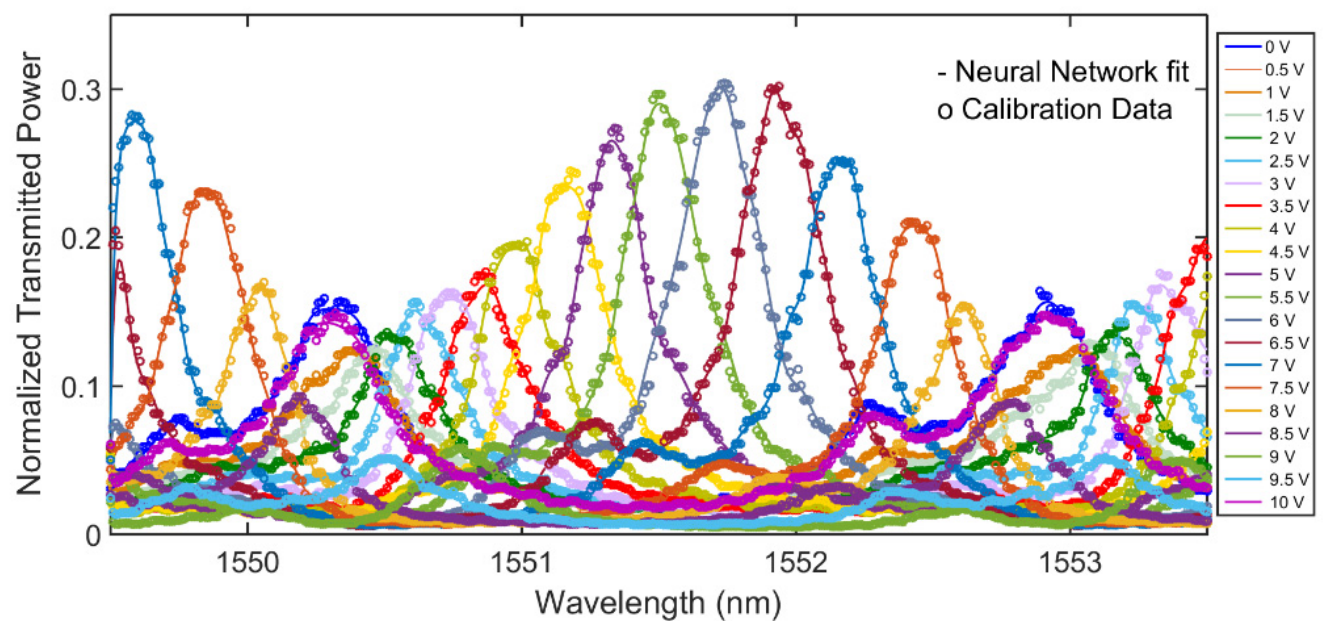

Fig. 3.2 Normalized calibration transmission spectra (circles) of the MRR for 21 different applied voltages (different colors) and the fitting of the normalized calibration data by the Neural Network (solid curves). The transmitted power at each wavelength is normalized with regard to the sum of the transmitted power signals over all the 21 applied voltages.

To test how precisely the calibrated wavelength meter estimates the wavelength of an optical input, some arbitrary wavelength, $\lambda_{\text {in }}$, within the calibration interval is injected and the transmission is recorded at the 21 settings for the heating voltages. As described in Sect. 3.1.2, a search algorithm is employed that finds the wavelength $\lambda_{\mathrm{e}}$ for which the NN calibration function predicts 21 transmission values with the minimum squared deviation from the 21 measured values. The wavelength of minimum deviation is displayed as the estimated wavelength, i.e., the wavelength as measured by the device. The estimated wavelength can then be compared with the actual injected wavelength, $\lambda_{\text {in }}$, as known from the readout of the laser module. For a comprehensive test across the entire spectral range, we perform the comparison sequentially with all of the 401 input wavelengths that were used before for calibration.

We note that in previous experiments [9] this test of the readout was carried out almost simultaneously with calibration (one millisecond delay), because the measurements aimed only on investigating the dependence of precision on the number of neurons and on the number of recorded data points. It thus remained open 
how far the smart readout is reproducible also over long time intervals that are of relevance for application.

In order to investigate the longer-term reproducibility, we have performed test measurements one week after calibration in a temperature-controlled laboratory (approximately $0.5{ }^{\circ} \mathrm{C}$ temperature stability over several days). A qualitative impression of the reproducibility of the recorded spectra can be obtained from Fig. 3.3,

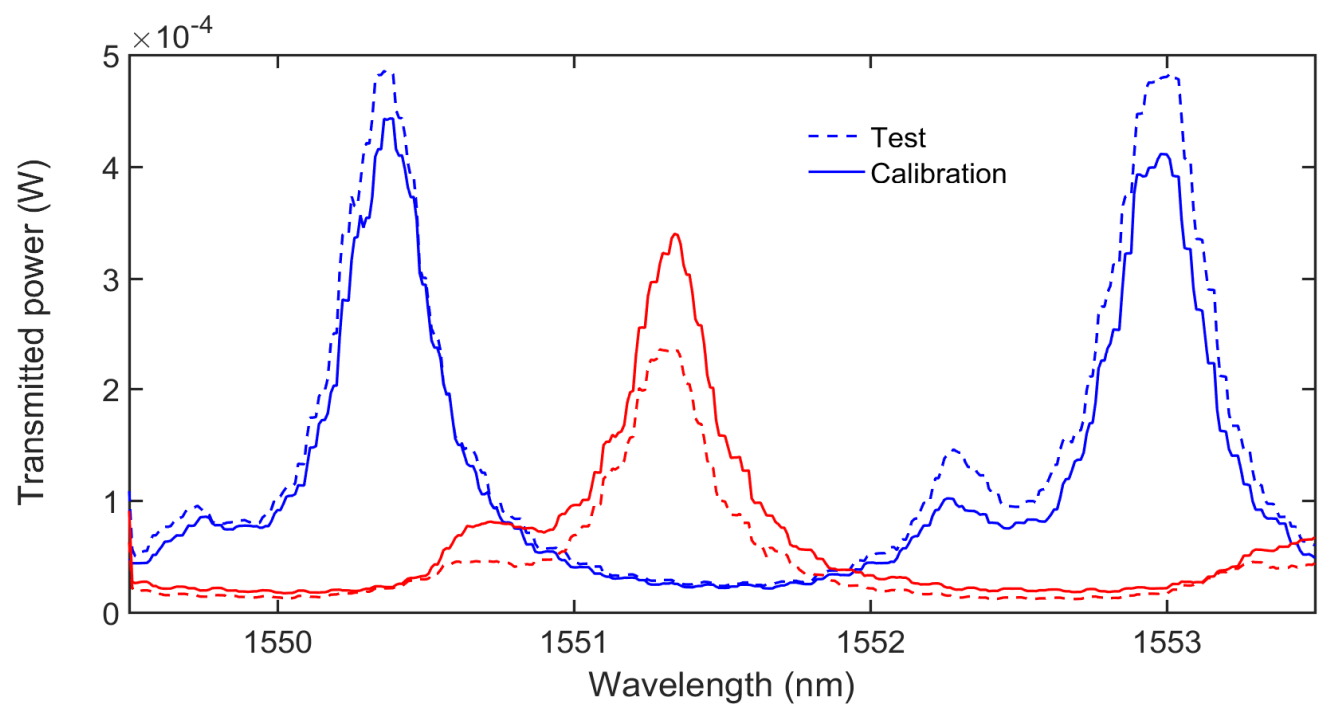

Fig. 3.3 Examples of transmission spectra of the MRR for two different applied voltages (blue: $0 \mathrm{~V}$, red: $5 \mathrm{~V}$ ). The thick lines represent the spectra acquired during the calibration; the dashed lines depict the data acquired during the test one week later.

where, for easier comparison, only two examples are shown. The agreement between the spectra recorded for calibration (solid curves) and a week later (dashed curves) is very good. This can be seen from the four peak wavelengths that have remained constant within only a small deviation of $20 \mathrm{pm}$. On the other hand, there are also some noticeable changes in the transmitted power at all main peaks. This might have been caused by a drift of alignment of the free-space coupling via lensed fibers.

For a quantitative evaluation of reproducibility across the entire spectral range (1549.5 nm to $1553.5 \mathrm{~nm}$ ) one week after calibration, the red line in Fig. 3.4 shows the estimated wavelength vs the input (supposedly unknown) test wavelength. For comparison, the straight blue line indicates the response of an ideal wavelength meter where the estimated wavelength is exactly equal to the input wavelength.

It can be seen that, for central wavelengths across a larger fraction of the FSR, the estimated values are very close to the ideal response, while outside the central FSR the estimation fails by giving large errors (outliers). The latter might be explained with the spectrally periodic transmission function of the MRR. The reason why the estimation works correctly only in the lower two-thirds of the FSR seems to be related to how the 


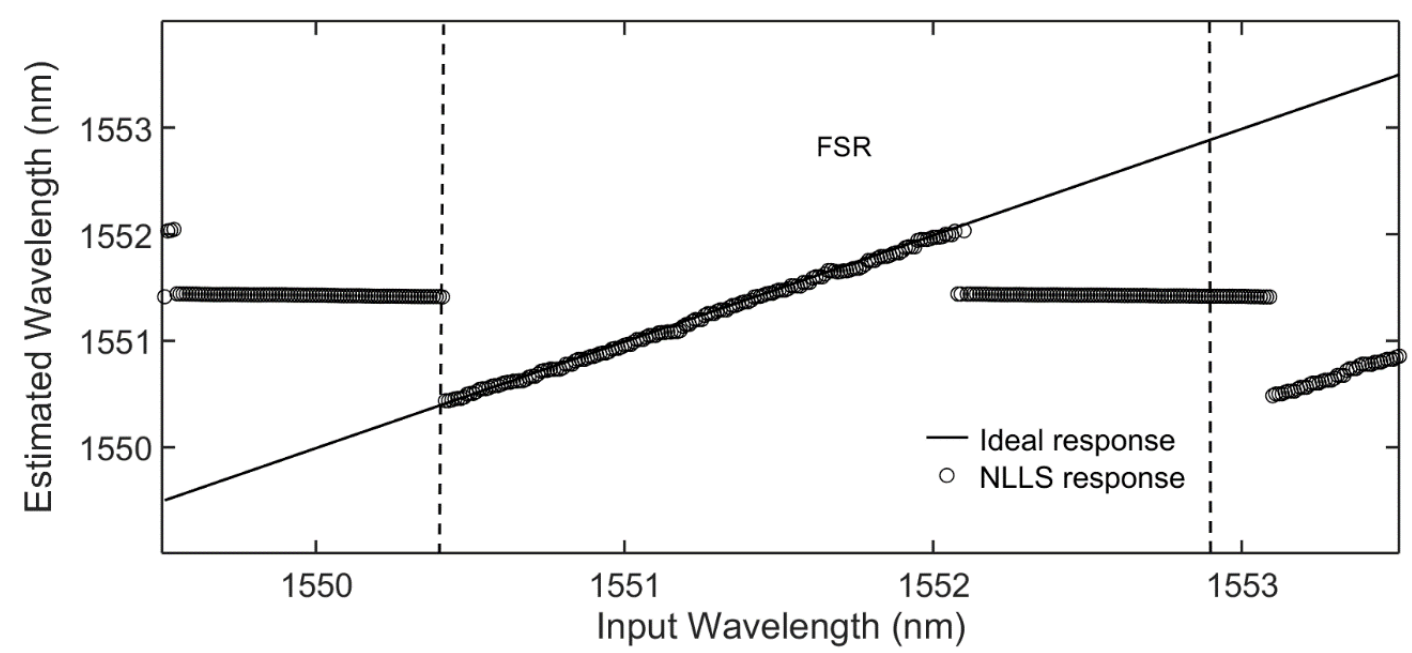

Fig. 3.4 Reproducibility of the output response of the wavelength meter after a one-week time interval. The circles represent the estimated wavelengths by the non-linear least square algorithm (NLLS) for the 401 input, supposedly unknown, wavelengths. The solid straight line indicates the response of an ideal wavelength meter across the free spectral range (FSR) of the MRR where the estimated (displayed) wavelengths are exactly equal to the known input wavelengths.

optimization algorithm searches for the minimum standard deviation between the 21 measured values and the 21 values predicted by the NN [13].For the wavelength range where the estimation error is small, i.e., from $1550.42 \mathrm{~nm}$ to $1552.07 \mathrm{~nm}$, we have plotted the deviation between the injected and the estimated wavelength in a histogram in Fig. 3.5. Determining the width of the histogram (FWHM) yields that the smart readout provides a spectral precision of about $50 \mathrm{pm}$. This resolution $(\approx 6 \mathrm{GHz})$

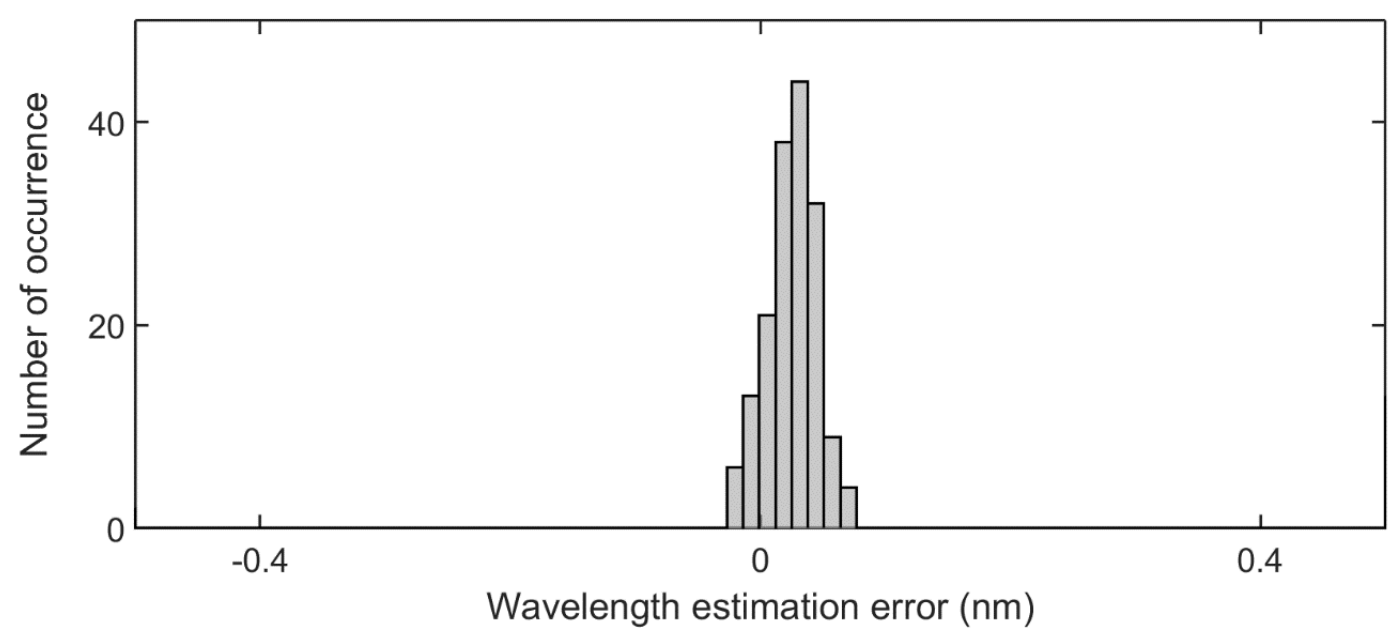

Fig. 3.5 Histogram of the estimation wavelength error calculated as the average difference between injected and estimated wavelengths in the central part of Fig. 3.4. The smart readout is capable to estimate the injected wavelengths correctly with $\approx 50$ pm precision.

corresponds, for instance, to the typical resolution of much larger, grating based optical spectrum analyzers. 
As a second important investigation, we measure to what extent the estimated wavelengths from the smart readout depend on external perturbations. In our experiments we selected a thermal perturbation in the form of a change in ambient temperature. The motivation for this choice is that such thermal perturbation would provide some maximum susceptibility (worst case) scenario because the control parameter used to mimic a larger number of $n$ devices is the temperature as well (temperature induced changes of the resonator length). A change in ambient temperature is expected to cause a common drift of the calibration of all the resonator transmission functions in Fig. 3.2. As was discussed in Sect. 3.1.2, a common drift should lead to a systematic error in the estimated wavelength that grows with the ambient temperature.

To induce a controlled change in ambient temperature, the wavelength meter setup was enclosed in an airflow-shielding box around the wavelength meter setup (indicated in the upper part of Fig. 3.1 as dashed frame), equipped with a suspended heater and a thermocouple for air temperature monitoring. The setup allowed increase of the ambient temperature and correspondingly that of the waveguide chip, with a delay of several minutes, by up to $6^{\circ} \mathrm{C}$. Measurements of the transmitted powers were carried out for a single input wavelength (again 21 voltages applied to the waveguide heater) while the ambient temperature was increased in steps of $1{ }^{\circ} \mathrm{C}$. In order to ensure that the waveguide chip follows the ambient temperature rise, we waited that

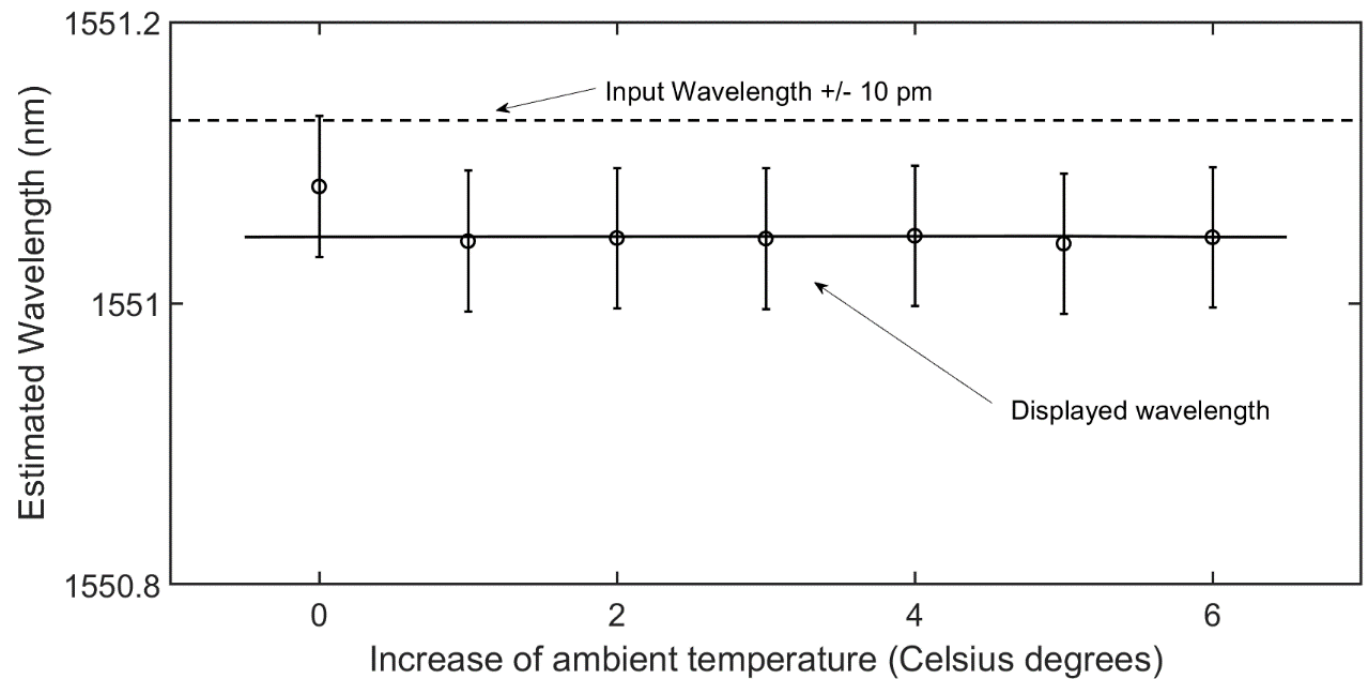

Fig. 3.6 Estimation of the smart readout for a single known input wavelength (dashed line). The circles are the estimated values for the known input wavelength at each temperature shift. The error bar is the estimation precision obtained with the long-term interval measurements, i.e. $\approx 50 \mathrm{pm}$. The solid horizontal line is drawn at the average estimated wavelength. 
the temperature displayed by the monitoring system was stable over tens of minutes and then performed the measurements which would take a few minutes.

Figure 3.6 shows the wavelength meter response for a fixed input wavelength $(1551.15 \mathrm{~nm})$ vs the increase in ambient temperature. Surprisingly, an approximately constant response was measured as is indicated by the horizontal blue line drawn at the average displayed wavelength. The lack of a temperature dependence of the wavelength meter response means that the smart readout appears unaffected by ambient temperature changes in the investigated range. Such result is quite surprising because, as mentioned above, the spectral effect of heating the ring resonator with the chip-based heaters should be the same as heating the resonator via the external heater. The transmission curves should tune exactly the same way, which is why the smart readout algorithm should not be able to distinguish between the different experimental realizations of the same waveguide temperature.

\subsubsection{Discussion}

One possible explanation of the observed immunity of the wavelength meter readout vs ambient temperature changes might be that the alignment stage onto which the chip is mounted possesses an unexpectedly large heat capacity. In this case the time needed to heat up the mount to a $1{ }^{\circ} \mathrm{C}$ higher ambient temperature would have to be longer than several tens of minutes. Unfortunately, the temperature rise vs time inside the waveguide resonator could not be monitored directly during the heating-up phases with sufficient precision via a shift of transmission resonances. The reason is an insufficient resolution of the MRR resonances in laser tuning spectra, as seen in Fig. 3.

The other hypothesis is that the waveguide temperature approximately followed the ambient temperature rise. In this case, the smart readout is somehow able to distinguish between the temperature change induced by the on-chip heaters and heating via the ambient temperature. This would only be possible if the experimental transmission spectra contain additional information beyond the theoretically expected transmission function of the MRR.

In order to test the latter hypothesis, we have calculated the temperaturedependent Fabry-Perot transmission of an ideal MRR with a physical model [14], for providing an ideal data set for NN calibration. To determine the temperaturedependent effective waveguide index, we calculate the mode field distribution from the temperature dependent material indices for $\mathrm{SiO}_{2}$ and $\mathrm{Si}_{3} \mathrm{~N}_{4}$ [15] and from the manufacturer specified waveguide cross section geometry. The physical waveguide length was taken from the fabrication specifications. 
In Fig. 3.7 the calculated transmission spectra of the ideal MRR model are displayed for 21 different model temperatures. Theoretically, a total temperature change from $0{ }^{\circ} \mathrm{C}$ to $195{ }^{\circ} \mathrm{C}$ is required to shift the spectrum by one FSR $(\approx 2.6 \mathrm{~nm})$. This corresponds to a wavelength shift of $14 \mathrm{pm} /{ }^{\circ} \mathrm{C}$, which is in good agreement with values reported in literature [15]. Considering that the overall ambient temperature change was $6^{\circ} \mathrm{C}$, the wavelength shift that this change should introduce is $\approx 85 \mathrm{pm}$.

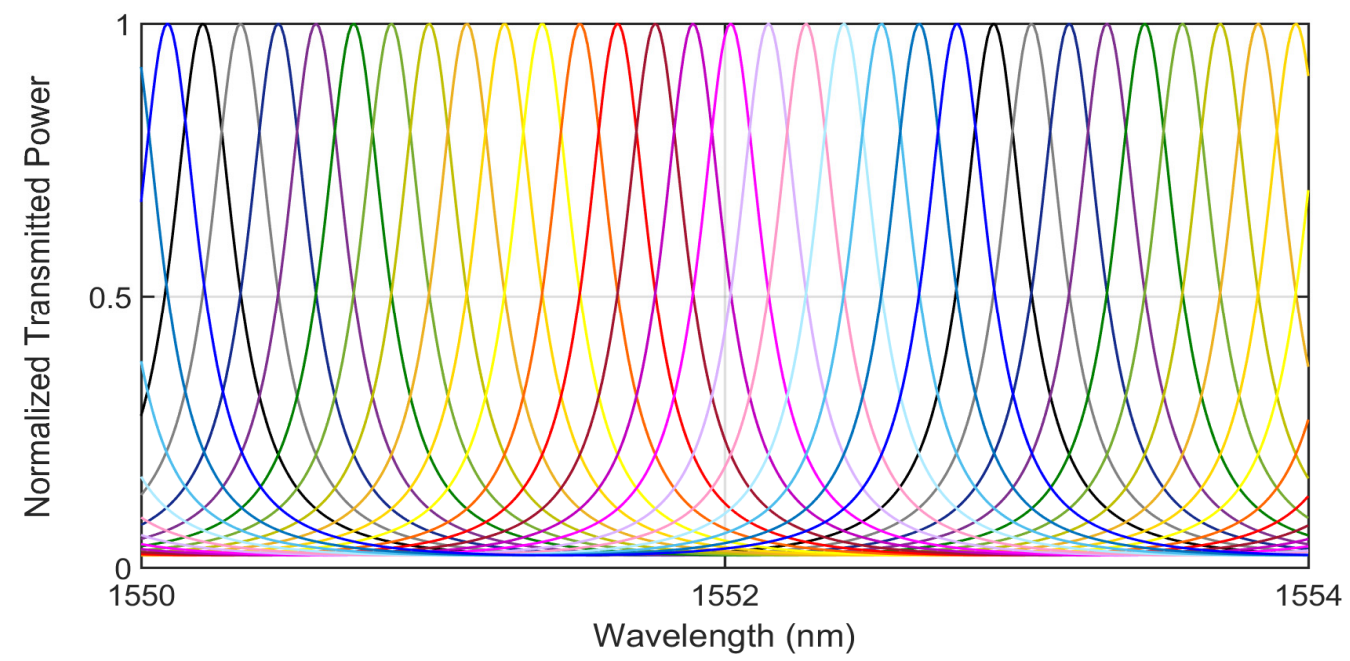

Fig. 3.7 Calculated transmission spectra of an ideal microring resonator at 21 different temperatures (different colors) equally spaced over an interval from $0{ }^{\circ} \mathrm{C}$ (black curve) to $195^{\circ} \mathrm{C}$ (blue curve).

To investigate the wavelength estimation capability of the smart readout for the ideal model resonator, we calibrated the NN and tested the readout based on the ideal transmission spectra from Fig. 3.7. The calibration was performed with the model data at 21 model temperatures, and the wavelength readout accuracy was tested with the same range of 401 wavelengths, as in the experiment. The comparison of the estimated wavelength vs the input wavelength is shown in Fig. 3.8. One can see that the estimation error within the central FSR is very small $(\approx 20 \mathrm{pm}$, about three times smaller than the experimental case). These results indicate a proper performance of the smart readout also with data from an ideal model resonator.

Finally, to investigate the sensitivity to changes of the temperature, we introduce a refractive index change corresponding to an increase of the ambient temperature of up to $6^{\circ} \mathrm{C}$, in steps of $1^{\circ} \mathrm{C}$. For each of these temperature offsets we generate the same set of spectra as in Fig. 3.7 that become slightly shifted from each other by 14 pm per step of $1{ }^{\circ} \mathrm{C}$. Finally, we use the smart readout to estimate the wavelength for fixed input wavelengths at the six ambient temperature configurations and from this derive the estimation error $(\approx 21.5 \mathrm{pm})$. 


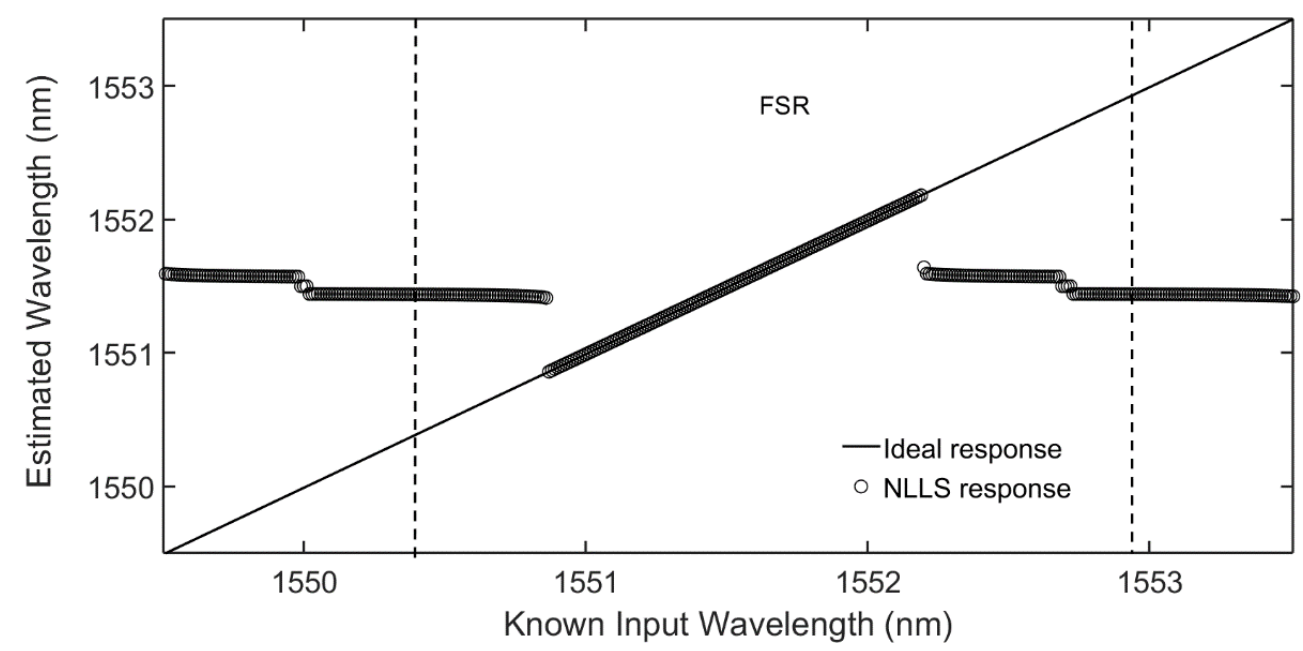

Fig. 3.8 Reproducibility of the smart readout for the case of a perfect ring resonator. The input and estimated wavelengths in the central spectral range agree within about $20 \mathrm{pm}$.

The results are summarized in Fig. 3.9. For clarity we report the case of a single input wavelength $(1551.74 \mathrm{~nm})$. It can be seen that, for an ideal MRR with the ideal Fabry-Perot response, all temperature changes detune the estimated readout wavelength (blue line), although the input wavelength is held constant (dashed line). This result confirms that the display of a smart-readout wavelength meter with a thermal control parameter cannot be immune to temperature induced perturbation, if the transmission function is ideal, such as with a model waveguide microring resonator.

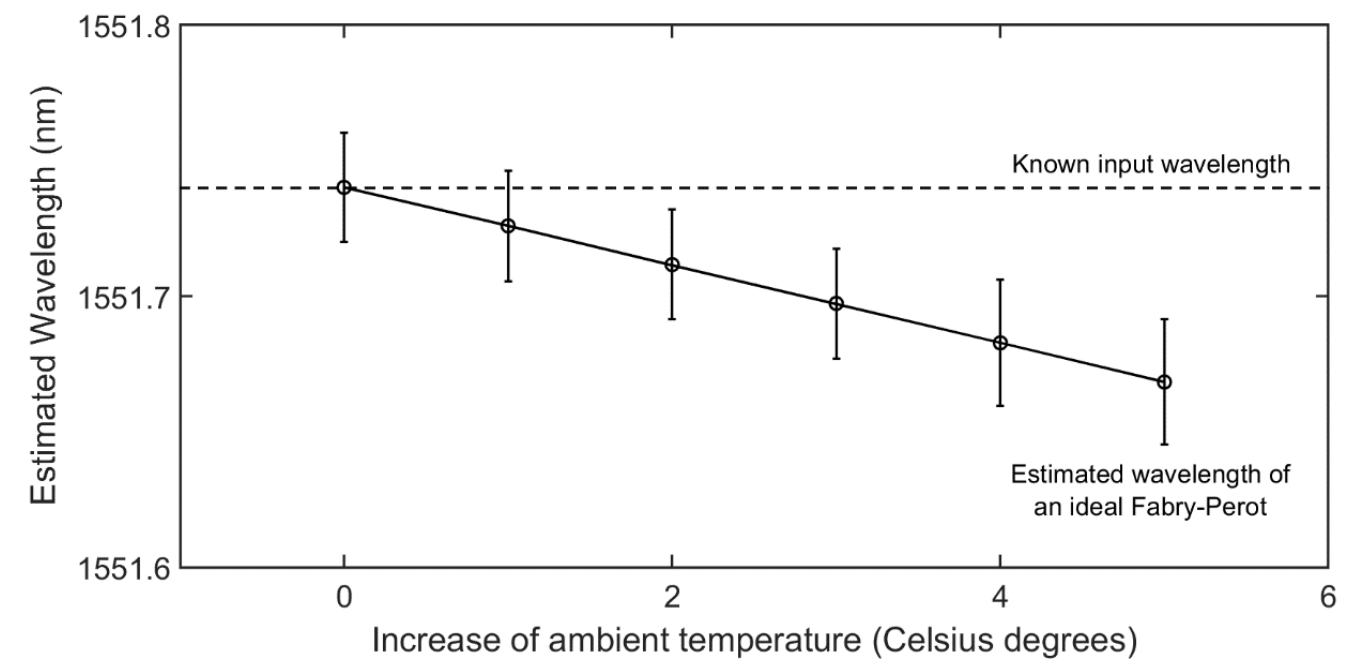

Fig. 3.9 Absence of temperature immunity with an ideal ring resonator. The circles, connected by a straight line, are the estimated values for the known input wavelength (dashed line) vs increasing temperature. The error bars are given by the finite estimation precision of the NN approach obtained at each room temperature configuration. 


\subsubsection{Conclusions}

The test time interval used here (about one week) is many orders of magnitude larger as compared to previous work. This interval is long enough that the wavelength meter display might have suffered from external perturbations, for instance, thermal drift or mechanical drifts in the setup. Nevertheless, we observe that the estimation precision is high, $\approx 50 \mathrm{pm}$, a value that is quite comparable (within about a factor of 3 ) vs measuring almost instantaneously (within 1ms) after calibration a). This shows, for the first time, that the investigated wavelength meter approach provides a very reproducible readout also over long time intervals, even if there are notable changes in the absolute transmitted power due to drifts in the fiber coupling alignment.

In the experiments we observe some temperature immunity of the wavelength meter. Based on a theoretically expected wavelength shift of $14 \mathrm{pm} /{ }^{\circ} \mathrm{C}$, there should have been an experimental wavelength shift of $85 \mathrm{pm}$. Compared to the experimental estimation error (about $50 \mathrm{pm}$ ) per single wavelength measurement, such a shift should have been clearly observable.

In order to start tracking the reasons for such immunity, we confirmed that measurements with an ideal resonator transmission function depending on a single temperature cannot provide immunity but displays the temperature drift as expected. A possible explanation that could not be excluded, due to lack of monitoring means for the waveguide internal temperature, is that the chip mount did not change its temperature noticeably, due to an unexpected high heat capacity.

The other possible explanation is that the experimental transmission spectra may have contained some independent information about the ambient temperature vs the thin-film heater induced temperature. To investigate this, more work is required; for instance, involving a set of MRRs that respond differently to a change of the ambient temperature than to a change of the waveguide-heater induced temperature. One might think, for example, of MRRs that possess different waveguide cross-sections. In this way, the mode confinement and thus the effective index of each MRR will differ from the others causing a different and distinguishable wavelength shift.

To conclude, our approach based on a single MRR and a smart readout algorithm is promising and open also for improvements into other respects. For instance, the covered wavelength range could be increased with waveguide circuits comprising a network of MRRs. Another option is increasing the resolution with ultra-low loss MRRs. Hybrid integration of passive and active waveguide platforms is also a 
promising field of application of our approach, such as for wavelength monitoring of hybrid lasers $[16,17]$.

\subsection{Range-extended wavelength meter based on double ring resonators in Vernier configuration}

In the previous section we have shown a wavelength meter of high precision $(\approx$ $50 \mathrm{pm})$, but its working range was limited to about two thirds of the FSR of the used MRR. Extending the working range of such a wavelength meter, i.e., extending its FSR, is crucial for, e.g., implementing filters and switching functionalities for telecommunication systems. As described in Chapter 2, a way to extend the FSR of a MRR-based system is to use two MRRs of slightly different radii in the form of a Vernier filter having a FSR that is much wider than the ones of the single MRRs.

\subsubsection{Experimental setup and results}

In order to explore the properties of on-chip Vernier filter wavelength measurements based on NN readout, a Vernier filter as used in laser experiments [18] was used for spectrometric experiments. Figure 3.10 shows a schematic of the waveguide chip containing two sequentially coupled MRRs of slightly different radii $\left(r_{1}=55 \mu \mathrm{m}\right.$ and $\left.r_{2}=59.6 \mu \mathrm{m}\right)$. The resonators are used in a mirror configuration, i.e., the drop port

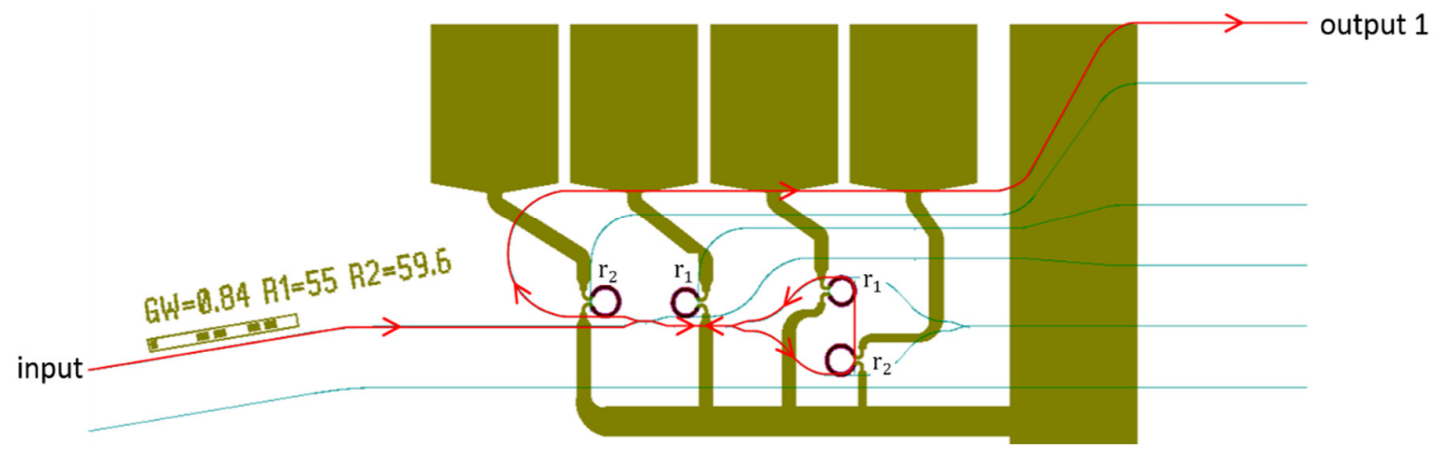

Fig.3. 10 Schematic of the Vernier filter in mirror configuration. The blue lines are optical waveguides. In red is the path of the light we investigate spectrally, from the input waveguide (on the left) to the record at output 1 (on the right). The purple circles indicate where the heaters are located, all around the ring resonators. In green the metal leads are shows, to apply two independently adjustable voltages to the two heaters.

output from the second ring is coupled back, via a Y-junction, into the input waveguide. The drop-port output, counter propagating with respect to the input signal, is extracted via a directional coupler (power coupling 5\%) and monitored at output 1 . 
For optical characterization of the chip and training of the smart readout algorithm, the same experimental setup is used as in Fig. 3.1. Light is coupled via a lensed fiber to the input port of the waveguide chip, which is tilted by an angle of 9 degrees, in order to minimize Fresnel reflection back into the fiber. Note that on its way to output 1 , the light is filtered by another MRR of $59.5 \mu \mathrm{m}$ radius, which is, however, not tuned. Therefore, the output 1 is the transfer function of the Vernier mirror except if a wavelength is injected that coincides with a resonance of that subsequent MRR.

To display the Vernier mirror transfer spectrum, Fig. 3.11 shows the normalized output power (output 1$)$ when the laser input $(0.5 \mathrm{~mW})$ is swept in wavelength from $1530 \mathrm{~nm}$ to $1580 \mathrm{~nm}$, in steps of $0.05 \mathrm{~nm}$, i.e., across 1001 input wavelengths. The spectrum has a shape that is typical for a Vernier filter and shows a Vernier free spectral range, FSRv, of about $43 \mathrm{~nm}$, which is approximately a factor of 15 larger than that of the single MRRs.

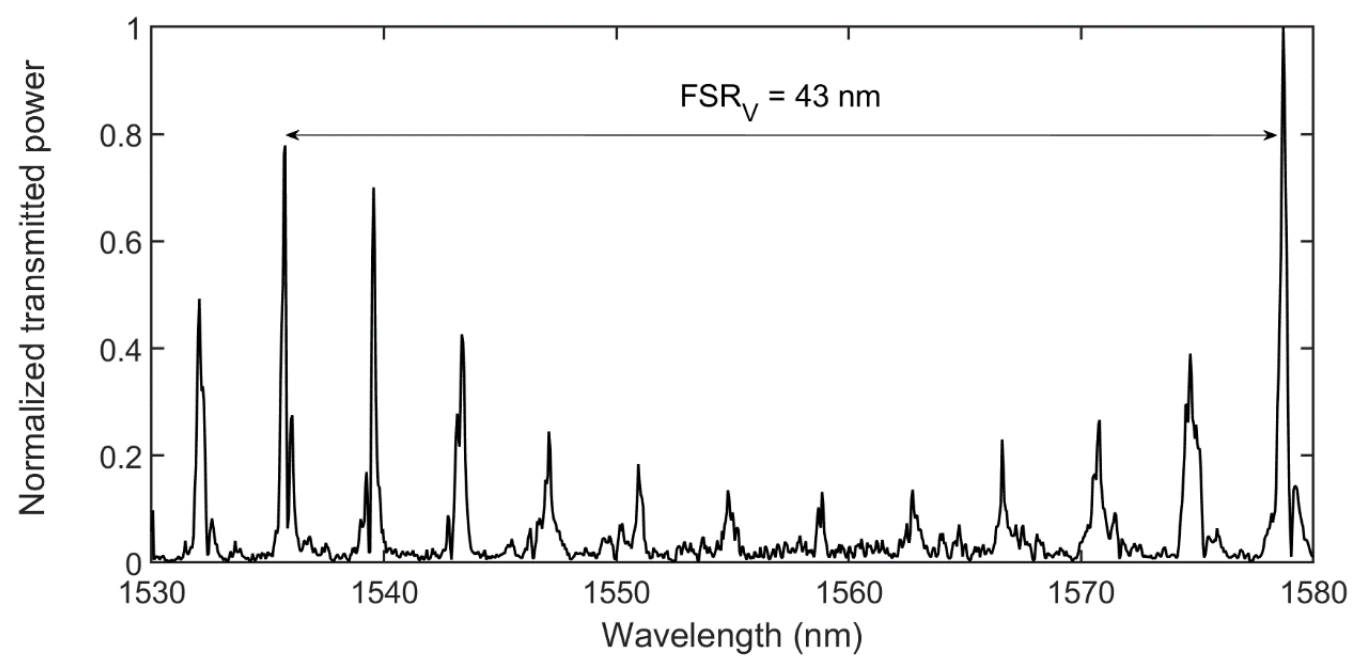

Fig. 3.11 Transmission spectrum of the Vernier mirror at output 1, normalized to the maximum transmission.

In order to train the smart readout algorithm, we acquire as the calibration data the transmitted power at each input wavelength for a set of applied heating voltages on each MRR. We heat the two MRRs independently by using two different power supplies, varying the applied voltage from 0 to $6 \mathrm{~V}$ in steps of $1 \mathrm{~V}$ for a total of 49 voltage settings. This choice of voltage values is motivated by the electrical properties and current limits for safe operation as follows. The resistance of the MRRs amounts to $R_{1}=170 \Omega$ and $R_{2}=180 \Omega$ for ring 1 and 2 , respectively. This set the maximum safe voltage to $\mathrm{V}_{1}{ }^{\max }=6.8 \mathrm{~V}$ and $\mathrm{V}_{2}{ }^{\max }=7.2 \mathrm{~V}$, considering a maximum allowed current of 40 $\mathrm{mA}$ per heater. 
Using 1001 input wavelength for each of the 49 voltage combinations, we train a NN of 200 neurons for a total of 10200 fit parameters. The expected advantage is that the number of fit parameters is much lower than the amount of data that they represent

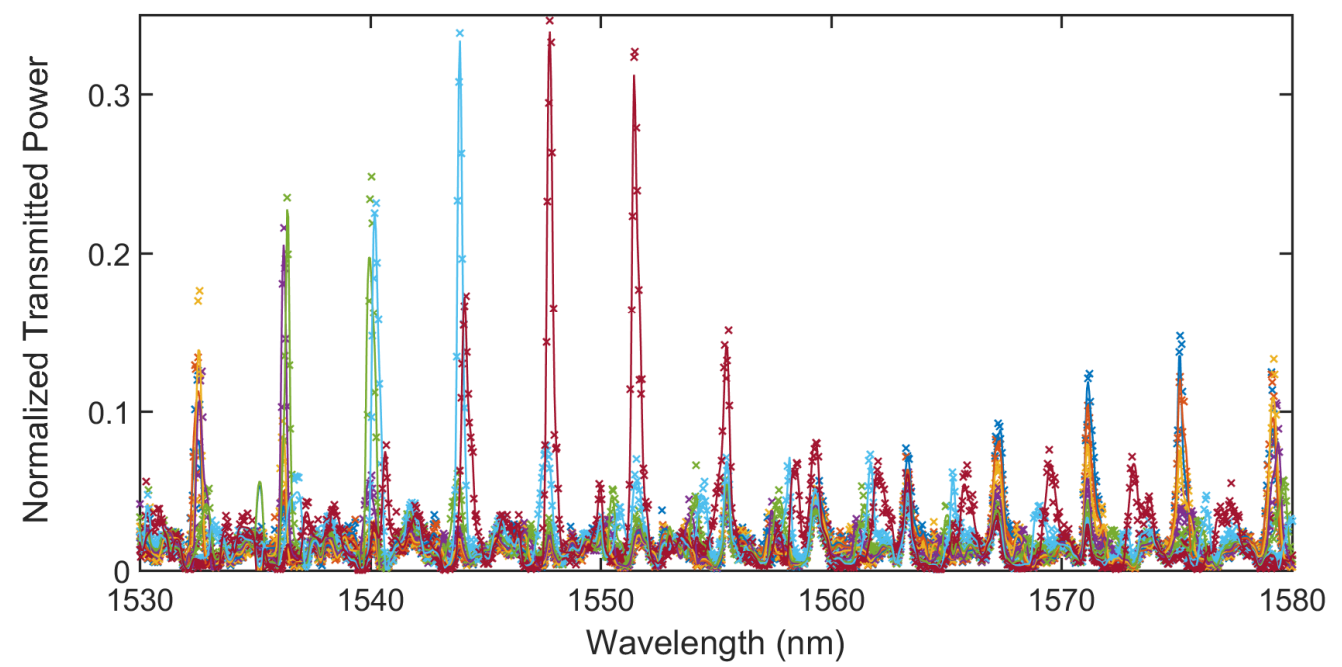

Fig. 3.12 Normalized calibration data for heater voltage of ring 1 fixed $(3 \mathrm{~V})$ and varying voltage on ring 2 . The ' $x$ ' represents the experimental data and the solid curves represent the $\mathrm{NN}$ fit.

(49049 transmission values), and that systematically searching through the analytically available NN fit function can save computational efforts. Fig. 3.12 shows that, after training, the NN fits the experimental transmission spectra well, which completes the calibration of the Vernier wavelength meter.

To test the capability of estimating unknown input wavelengths, specifically with regard to an extended wavelength range, we first investigate the reproducibility of the

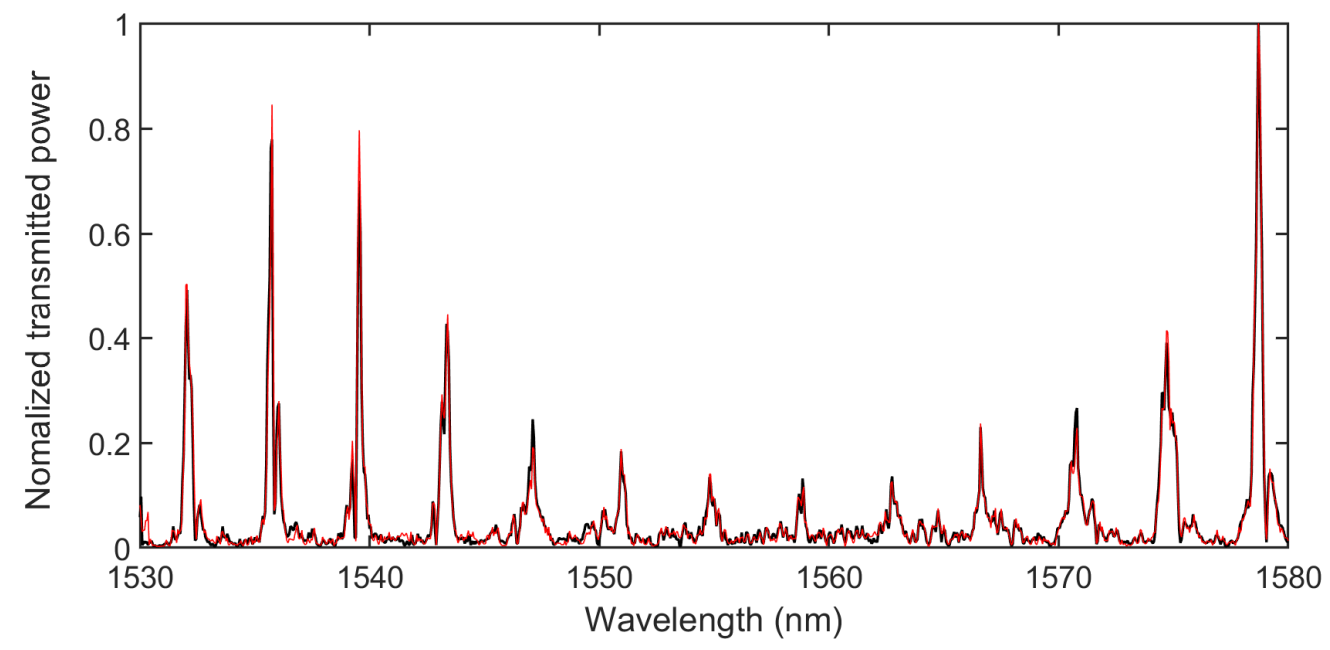

Fig. 3.13 Normalized transmission spectra of the calibration data set (black) and the test data set (red) acquired 4 days after calibration. 
wavelength meter response, by performing the same experimental measurements four days after calibration. As shown in Fig. 3.13 at a typical example, the recorded test spectra overlap well with the calibration spectra. Compared to calibration, only slight variations in the peak power of the test resonances can be noticed, which is likely due to a drift in the lensed fiber coupling.

For the estimation, we apply three different methods. The first, as the one used for the single MRR based wavelength meter (section 1.1), is based on searching for the minimum of the nonlinear cost function (mean-square deviation) using a local minimum optimization algorithm as follows. After using a known input test wavelength and having measured the transmission values for all 49 heater settings, the algorithm searches for the minimum deviation between the 49 measured values and 49 values predicted by the NN by local variation of the estimated wavelength. The term "local" means that the search begins with a fixed estimation wavelength in the center of the calibration range and that steps to adjacent wavelengths are used, in a direction where the cost function value becomes smaller, to find the estimation wavelength that minimizes the cost function.

Figure 3.14 shows, for the 1001 known input wavelengths, the wavelengths (circles) estimated by the NN using the local search method (NLLS). It can be seen that the wavelength range of a close-to-ideal response (blue line, average deviation of data points $109 \mathrm{pm}$ ) is only about $1.8 \mathrm{~nm}$ wide. When comparing this to range obtained with a single MRR (1.4 nm, see previous section), there is hardly any increase in range, despite the much wider FSR of the Vernier filter.

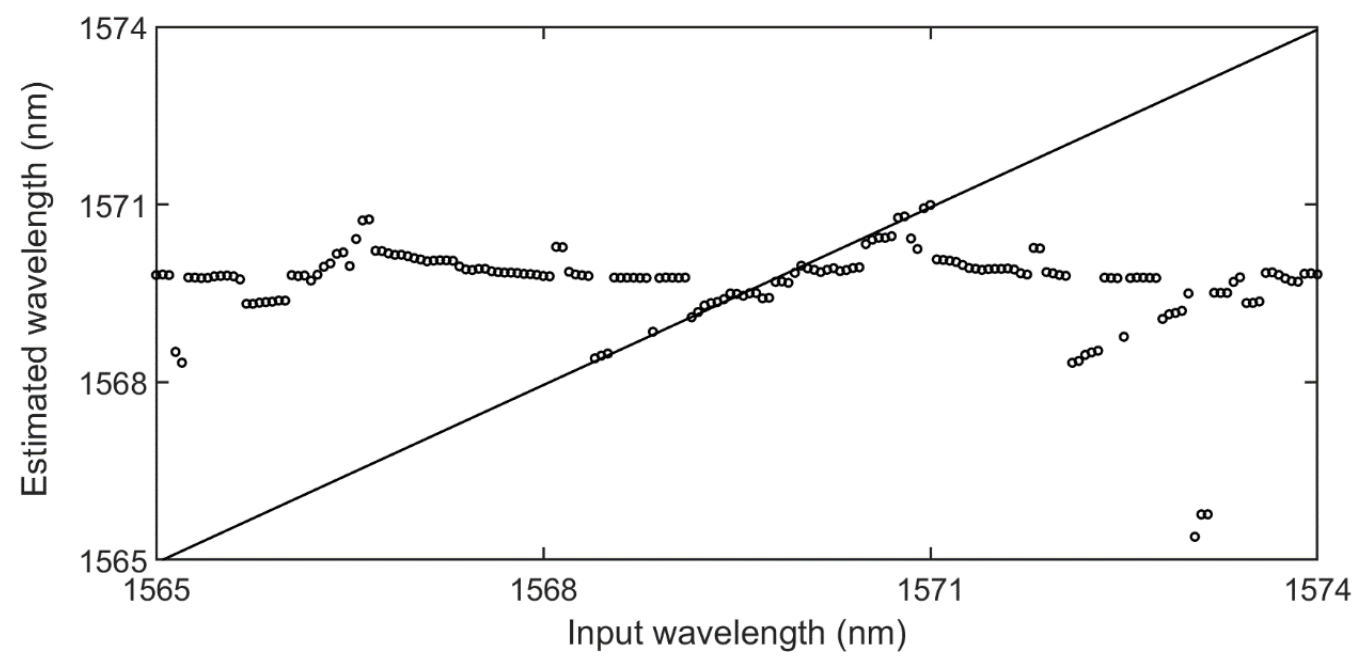

Fig. 3.14 Estimated wavelengths by the local optimization algorithm (circles) versus the known input wavelength. The blue line is a guide to the eye and represents the response of a perfect device that estimate exactly the wavelength of the known input light. 
The reason why the estimation range does not experience the same enlargement as the FSR may be found in what manner the search algorithm moves along possible estimation wavelengths in order to minimize mean-squared error (MSE) between 49 measured and NN-predicted transmission values.

In order to identify the problem, the observed small working range, Fig. 3.15

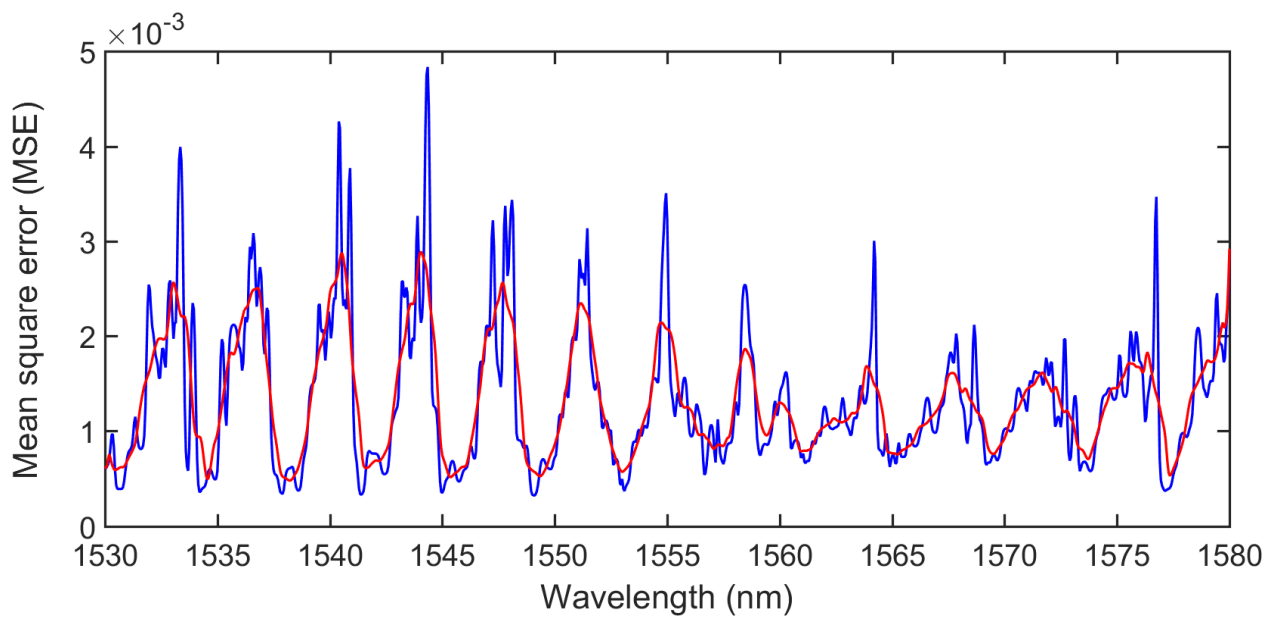

Fig. 3.15 Mean square error between the 49 experimental transmission values of an arbitrary input test wavelength and the neural-network predicted values of the calibrated ones (blue trace). A smoothed version of the MSE function is shown in red. We notice that the MSE function, which is the objective function (cost function) that the wavelength search algorithm tries to minimize, contains many local minima and not even a clear global minimum.

shows the MSE for all test wavelengths across the FSR of the Vernier filter. It can be seen that the MSE varies approximately periodically with test wavelength and shows many minima. These minima can be understood from the presence of periodically spaced side peaks in the Vernier filter spectrum. From the appearance of multiple minima in the MSE function it becomes clear why the local-minimum search algorithm had failed to extend the range of the wavelength meter namely, that a local search ignored possibly smaller MSE values at largely different wavelengths.

The second method under test was using global minimization. For this purpose, the algorithm was set to search for the absolute lowest of all MSE values. Figure 16 shows the results obtained with the global search approach. It can be seen that the estimation range indeed increases; however, again the range is only slightly wider (2 $\mathrm{nm}$ with global search, instead of $1.8 \mathrm{~nm}$ with local search). At the same time there is also a drop of estimation precision (154 pm instead of 109 pm, respectively). 


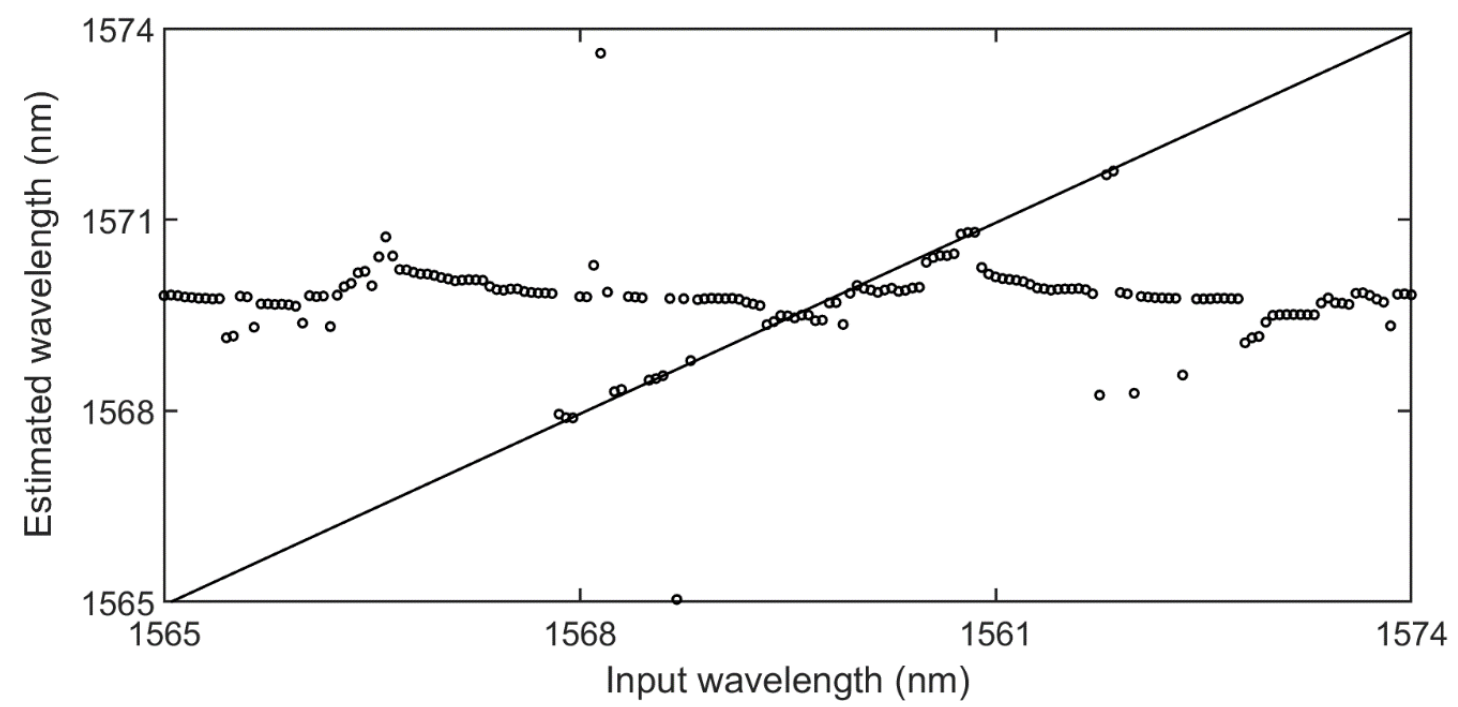

Fig. 3.16 Estimated wavelengths by the global optimization algorithm (circles) versus the unknown input wavelength. The blue line is a guide to the eye and represents the response of a perfect device that estimates exactly the wavelength of the unknown input light.

The third method investigated is what is commonly called look-up table. In this case, the wealth of calibration data in not represented (replaced) by an approximate

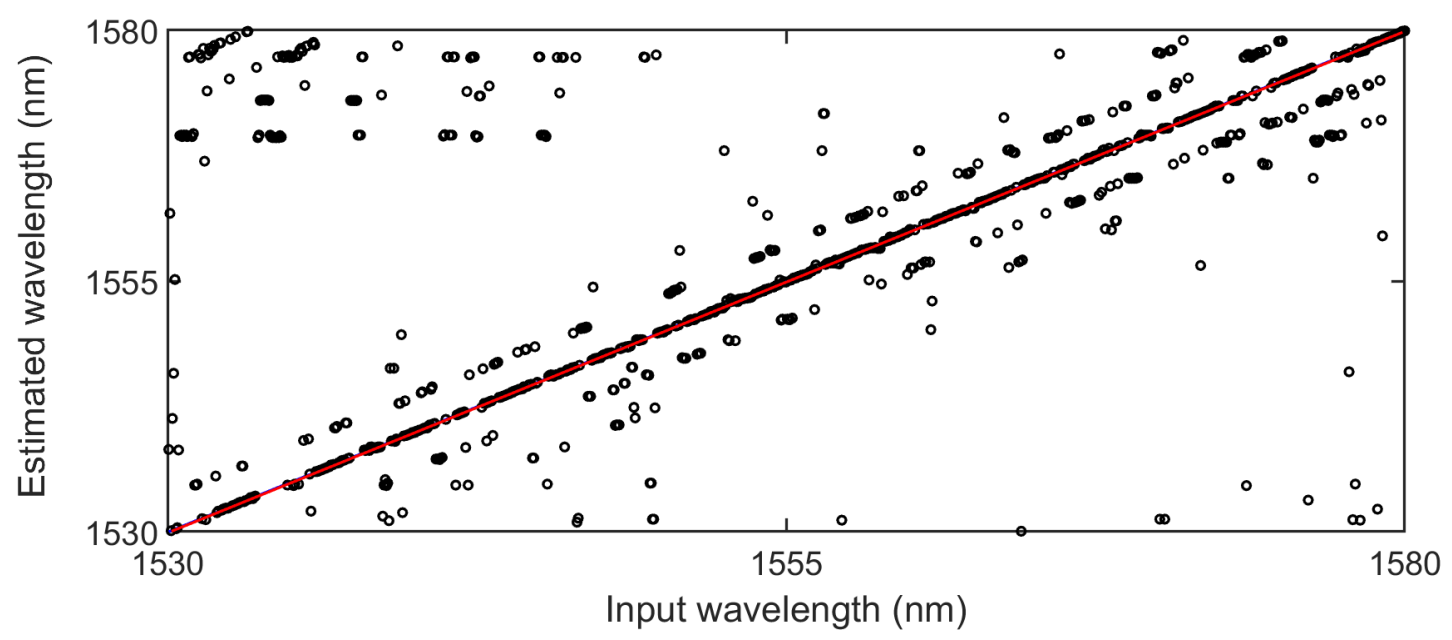

Fig. 3.17 Estimated wavelengths by the "look-up table" method (circles) versus the unknown input wavelength. The red line is a guide to the eye and represents the response of a perfect device that estimate exactly the wavelength of the unknown input light.

$\mathrm{NN}$ fit function, but the original transmission values measured during calibration are taken for comparison with those from later test measurement. In other words, the wavelength search algorithm looks for the global minimum of the MSE between the transmission with a known test wavelength and the transmission with all 1001 wavelengths used in calibration. The estimated wavelengths obtained via the look-up 
table method are plotted in Fig. 3.17. It can be seen that the range of the wavelength meter becomes clearly extended to a width of about $26 \mathrm{~nm}$ because the majority of data points (wavelength estimates) lies on the straight line that represent a perfect wavelength response. This range amounts to about two thirds of the FSR of the Vernier filter. Nevertheless, there are also a large number of wrong wavelength estimates (outliers) that partly follow some systematic variation with the test wavelength. From Fig. 3.17 it can be noticed clearly that the data not along the ideal response are actually in a step-like distance from it, i.e., aligned in parallel with deviations that are multiples of $\sim 4 \mathrm{~nm}$, which is consistent with the distance between the resonances of the Vernier mirror, as shown in Fig. 3.11. Another observation is that the estimation precision is somewhat less than with training a NN, amounting to about $175 \mathrm{pm}$.

\subsubsection{Conclusions}

We have investigated a novel type of smart wavelength meter based on two MRRs coupled in a Vernier configuration which shows an extended FSR (43 nm) compared to the one of a single MRR $(2.6 \mathrm{~nm})$. For the smart readout we used a neural network (NN) comprising 200 neurons.

The range over which the NN-based wavelength estimation is working well, unfortunately does not show the expected enlargement proportional to the increased FSR, but it actually increases only slightly as compared to that of a single MRR. This refers to both a local and global search for minimizing the cost function, i.e., the MSE between measured and NN-predicted transmission values.

In order to achieve further improvements, we compare with a non-neuralnetwork-based approach in the form of a look-up table. In this case we find a much larger estimation range $(26 \mathrm{~nm})$, although the estimation precision is lower than with the other methods.

In conclusion, estimating unknown input wavelengths with integrated optical waveguide circuits is possible with various methods, involving both a neural network (so-called smart readout) approach as well as a standard readout in via a look-up table. The measurement analyses presented here suggest that various central improvements need first to be pursued before such devices can be employed in applications.

A first improvement is to base next experiments on fully packaged devices, i.e., where input and output fibers as well as voltage supplies are permanently connected. This would prevent, e.g., uncontrolled effects entering via lensed fiber coupling and increase the optical throughput for higher signal to noise ratio. 
A second point refers to find improvements with search algorithms. For instance, it seems that the various approaches (neural network vs look up table) have complementary strengths, in terms of precision and wavelength range. It may thus be possible to combine the strengths, such as using lookup methods for coarse wavelength estimation, followed by $\mathrm{NN}$ representation with local search for subsequent refinement. Other options might consist simply in better search algorithms, instead of local and global. For instance, so-called shooting algorithm have shown promising results in biological networks [19].

A third point of improvement is to extend the type of waveguide circuits. For instance, the microring resonators used here appear to be a good choice for obtaining high resolution. This can be concluded from the very high quality-factors that can be exploited in multiple roundtrips resonator [11, 20,21]. On the other hand, making use of high-quality resonators also imposes that there is no wavelength characteristic change of the transmission over a large part of the FSR (i.e. between the resonant wavelengths). In the specific case of sequential resonators, as was presented above with two resonators, most of the combined heater settings yield only small optical transmission, while most of the optical signal is lost to other ports. These disadvantages would be removed by waveguide circuits where resonators are arranged in parallel, fed by the same bus waveguide, and where all resonator transmissions are red-out in parallel with multiple detectors. The problem of the partly lacking wavelength characteristic response of resonators may be alleviated with trees of arm-length misbalanced Mach-Zehnder interferometers [22]. These would allow, similar to so-called arrayed waveguide arrays, a course but broad wavelength estimate, possibly followed by refined precision through resonators. 


\section{References}

[1] Rodriguez-Fernandez, C., Manzano, C. V., Romero, A. H., Martin, J., MartinGonzalez, M., Morais de Lima M. Jr. and Cantanero, A., "The fingerprint of Terich and stoichiometric $\mathrm{Bi}_{2} \mathrm{Te}_{3}$ nanowires by Raman spectroscopy," Nanotechnology 27, 075706 (2016).

[2] Early, D. A., Reth, A. D. and Rodriguez-Alvarez, O. I., "Spacecraft pointing stability constraints and instrument disturbance limits for optical remote sensing satellites," Proc. SPIE 5234, 560-571 (2004).

[3] Dey, R., Doylend J., Ackert, J., Evans, A., Jessop, P. and Knights, A., "Demonstration of a wavelength monitor comprised of racetrack-ring resonators with defect mediated photodiodes operating in the C-band," Optics Express 21, 23450-23458 (2013).

[4] Okamoto, H., Haraguchi, M., Fukui, M. and Okamoto, T., “Optical filtering by microring resonators," Jpn. Journal of Applied Physics 42, 2692-2698 (2003).

[5] Nitkowski, A., Chen, L. and Lipson, M., "Cavity-enhanced on-chip absorption spectroscopy using microring resonators," Optics Express 16, 11930-11936 (2008).

[6] Ramachandran, A., Wang, S., Clarke, J., Ja, S. J., Goad, D., Wald, L., Flood, E. M., Knobbe, E., Hryniewicz, J. V., Chu, S. T., Gill, D., Chen, W., King, O. and Little, B. E., "A universal biosensing platform based on optical micro-ring resonators," Biosensors and Bioelectronics 23, 939-944 (2008).

[7] Spencer, D. T., Bauters, J. F., Heck, M. J. R., Bowers, J. E., “Integrated waveguide coupled $\mathrm{Si}_{3} \mathrm{~N}_{4}$ resonators in the ultrahigh-Q regime," Optica 1, 153-157 (2014);

[8] Worhoff, K., Heideman, R. G., Leinse, A. and Hoekman, M., “TripleX: a versatile dielectric photonic platform," Advanced Optical Technologies 4, 189-207 (2015).

[9] Oldenbeuving, R. M., Song, H., Schitter, G., Verhaegen, M., Klein, E. J., Lee, C. J., Offerhaus, H. L. and Boller, K.-J., "High precision wavelength estimation method for integrated optics," Optics Express 21, 17042-17052 (2013).

[10] Padmaraju, K. and K. Bergman, "Resolving the thermal challenges for silicon microring resonator devices". Nanophotonics, p. 269 (2014).

[11] Roeloffzen, C.G.H., et al., "Low-Loss $\mathrm{Si}_{3} \mathrm{~N}_{4}$ TriPleX Optical Waveguides: Technology and Applications Overview". IEEE Journal of Selected Topics in Quantum Electronics 24(4), p. 1-21 (2018).

[12] Morichetti, F., Melloni, A., Martinelli, M., Heideman, R. G., Leinse, A., Geuzebroek, D. H. and Borreman, A., "Box-shaped dielectric waveguides: a 
new concept in integrated optics?," Journal of Lightwave Technology 25, 25792589 (2007).

[13] Orgun M.A. and Thornton J. (eds) [AI 2007: Advances in Artificial Intelligence. Lecture Notes in Computer Science], Springer Berlin - Heidelberg, 100-109 (2007).

[14] Kedia, J. and Gupta, N., "Mathematical modeling of ring resonator filters for photonic applications," IJARCSEE 2, 123-128 (2013).

[15] Arbabi, A. and Goddard, L. L., "Measurements of the refractive indices and thermos-optic coefficients of $\mathrm{Si}_{3} \mathrm{~N}_{4}$ and $\mathrm{SiO}_{x}$ using microring resonances," Optics Letters 38, 3878-3881 (2013).

[16] Fan, Y., Oldenbeuving, R. M., Klein, E. J., Lee, C. J., Song, H., Khan, M. R. H., Offerhaus, H. L., van der Slot, P. J. M. and Boller, K.-J., “A hybrid semiconductor-glass waveguide laser," Proc. SPIE 9135, 91351B (2014).

[17] Fan, Y., Epping, J., Oldenbeuving, R. M., Roeloffzen, C. G. H., Hoekman, M., Dekker, R., Heideman, R. G., van der Slot, P. J. M. and Boller, K.-J., “Optically integrated InP-Si3 $\mathrm{N}_{4}$ hybrid laser," IEEE Photonics Journal 8, 1505111 (2016).

[18] Oldenbeuving, R.M., et al., “25 kHz narrow spectral bandwidth of a wavelength tunable diode laser with a short waveguide-based external cavity". Laser Physics Letters 10(1), p. 015804 (2012).

[19] Binder, S.C., E.A. Hernandez-Vargas, and M. Meyer-Hermann, Reducing complexity: An iterative strategy for parameter determination in biological networks. Computer Physics Communications 190, p. 15-22 (2015).

[20] Spencer, D.T., et al., "Integrated waveguide coupled Si3N4 resonators in the ultrahigh-Q regime". Optica 1(3), p. 153-157 (2014).

[21] Taddei, C., et al., "High-Selectivity On-Chip Optical Bandpass Filter With Sub100-MHz Flat-Top and Under-2 Shape Factor". IEEE Photonics Technology Letters 31(6), p. 455-458 (2019).

[22] Horst, F., et al., "Cascaded Mach-Zehnder wavelength filters in silicon photonics for low loss and flat pass-band WDM (de-)multiplexing". Optics Express 21(10), p. 11652-11658 (2013). 


\section{A reconfigurable linear optical circuit}

\subsection{Introduction}

Integrated photonic devices providing linear optical circuits (LOCs) based on multistage MZI architectures have received increasingly strong interest in recent years. The repetition of a reconfigurable unit cell, i.e., a tunable MZI, enables the realization of a large-scale reconfigurable and programmable integrated LOC capable of processing the incoming light on the same device for different applications. Programmable LOCs, which we briefly name photonic processors here, have been integrated using various different material platforms such as $\mathrm{Si}_{3} \mathrm{~N}_{4}$, silicon-on-insulator (SOI) and doped silica. Quite a variety of applications are envisioned, e.g., microwave filters [1-3], quantum information processing [4-6] and optical neural networks [7].

The response and configurability of a LOC, specifically the capability to perform a desired optical transformation, depends critically on the tuning response of each individual unit cell with regard to an external control. Unfortunately, in complex and large multi-stage architectures, the response of each particular unit cell is usually not directly accessible with measurements, because the measured output also depends strongly on the response of the other unit cells, among which the particular cell of interest is embedded. In this situation, the effects of undesired fabrication errors in each single unit may multiply in a cascade, strongly affecting the overall LOC response. This can make it difficult or impossible to operate a processor as intended, due to lack of knowledge regarding its internal properties. The named problem is not only severe but also general. The reason is that in any LOC it is fundamentally impossible to avoid fabrication errors or deviations. Optically these show up, for instance, as losses or imperfect directional couplers (DCs), which limit the functionality of the processor, for instance, the range of reconfigurability.

Various methods have been developed in order to operate such processors via avoiding the problematic task of characterizing all of the internal, single unit cells. However, these methods require local monitoring of the light and active feedback [8, 9] and they have been demonstrated only for small dimensions of the processor [10]. 


\section{A reconfigurable linear optical circuit}

In the case that the tuning response of the individual cells is not well-know it has been demonstrated that imperfect DCs can be compensated by specific architectures [11], i.e., by adding extra components that may be imperfect as well. For instance, optical losses can be balanced by more symmetric architectures in the optical paths, so that the transmitted light experiences the same number of optical components (beam splitters and phase shifters) for any mode [12].

Nevertheless, such avoiding of the full characterization of a LOC (self-configuring approach [9]) or compensating the fabrication errors with extra components can substantially increase the power consumption and the footprint of the processor, as local monitoring of the light, feedback loops and more unit cells are required.

In the other case that a full characterization of all unit cells can be achieved, i.e., where based on individual calibration each cell can be individually controlled in a known manner, it may be possible that an optimization of the processor settings can be performed: without adding any extra component, it may be possible to improve the response of an imperfectly fabricated processor, with the goal to approach that of a perfect processor without fabrication errors.

In order to explore these possibilities, this chapter investigates a full characterization and calibration of a large ( $8 \times 8$ modes) reconfigurable LOC based on $\mathrm{Si}_{3} \mathrm{~N}_{4}$ waveguides, followed by investigations of the processor performance and the options for optimized settings. In section 4.2 we describe the multi-stage architecture of the processor and the experimental setup. Section 4.3 contains the results of the full optical characterization of our processor. The latter is based on the characterization and calibration of all individual unit cells, followed by investigations of the fidelity with which arbitrary (Haar random) unitary optical transformations can be realized. In the last section we present first results towards optimization of the processor's response.

\subsection{Multi-stage architecture and experimental setup}

Figure 4.1 shows the schematic of the multi-stage architecture of our processor, consisting of 64 cascaded unit cells forming an $8 \times 8$ (input-output) channel rhomboidal network. Each unit cell comprises a tunable MZI, implementing a thermo-optic tunable beam splitter, preceded by a phase shifter in one of the input waveguides (upper input waveguide in inset of Fig. 4.1). The network, as implemented with integrated optical waveguides on a chip, has a rectangular shape. Due to this implementation, the optical paths from each input to each output waveguide (mode) and from a unit cell to its two consecutives are not all exactly equally long. This causes 
the optical losses experienced along the 64 different light paths to be slightly different, as light goes through a different amount of unit cells depending of the chosen path. An optical architecture promising equal path losses has been proposed in [12].

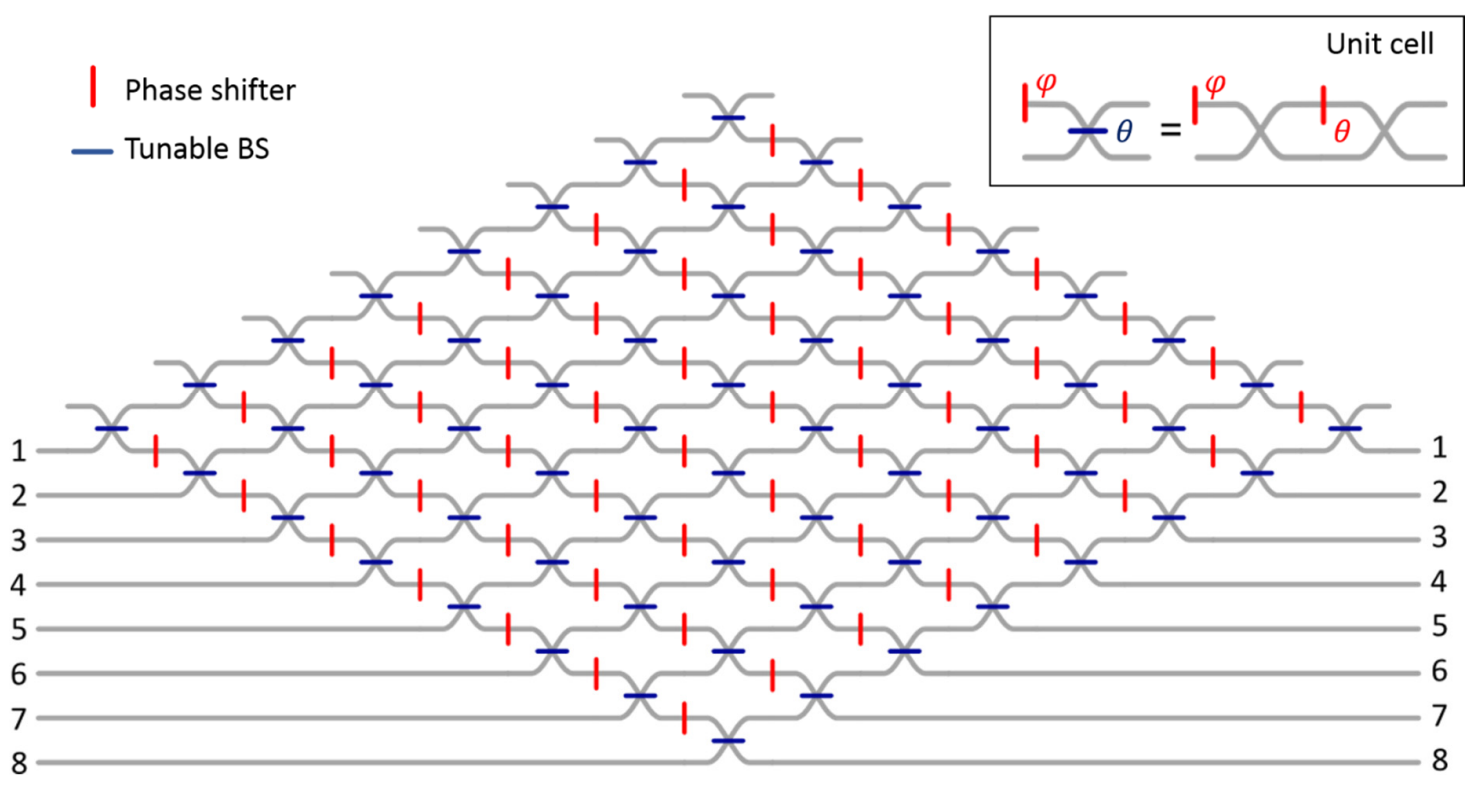

Fig. 4.1 Schematic of the processor's architecture given by the repetition of a reconfigurable unit cell (inset) comprising a phase shifter (in red) and a tunable beam splitter (in blue). Tunable beam splitters are realized as a Mach-Zehnder interferometer with a phase shifter in one arm.

The multi-stage architecture shown in Fig. 4.1 is topologically equivalent to a double triangular Reck scheme [13], where the bottom and top triangle correspond to a Reck scheme of $8 \times 8$ modes. Theoretically, a LOC built perfectly following the Reck scheme enables the realization of any unitary transformation using only linear optical elements such as phase shifters (PS) and tunable beam splitters (TBS). A perfectly implemented Reck scheme can thus be named universal.

However, whether a particular, i.e., fabricated LOC is able to implement any unitary transformation, i.e., whether it is universal, depends on the realized tunability of the PSs and TBSs as investigated in this chapter.

Further describing the realization of the processor, each solid line in Fig. 4.1 corresponds to an optical waveguide. Access to the whole LOC is given by the 8 inputs modes at the lower triangle. To ensure low propagation losses in the order of 0.2 $\mathrm{dB} / \mathrm{cm}$, a small footprint in the order of 1 by $2 \mathrm{~cm}$, and for providing single-mode propagation at telecom C-band wavelengths (around $1550 \mathrm{~nm}$ ), a double-stripe waveguide cross section is chosen (see Section 2.1.2). The total on-chip propagation losses are found to be less than $40 \%(-2.2 \mathrm{~dB})$. This value is measured along the longestpossible on-chip geometrical path length (about $10 \mathrm{~cm}$, along 15 TBSs) and 
corresponds to about $0.22 \mathrm{~dB} / \mathrm{cm}$. An array of polarization-maintaining (PM) fibers is bonded to the chip to give optical access to the waveguides. The losses in these fiberto-waveguide couplings are still relatively high, about $3 \mathrm{~dB}$ per coupling, because the current chip is not equipped with waveguide tapers. The processor as a whole is temperature-stabilized by a Peltier element. Independent thermal tuning of the 120 (chromium-gold) thermo-optic phase shifters is accomplished via USB-controlled heater voltage drivers. The entire assembly comprising the chip, fiber arrays and electronics, is packaged into a single portable case with a USB connection and power socket at its back, and with 16 FC/PC fiber connectors at the front panel.

Figure 4.2 shows a schematic of the experimental setup. For characterization of the processor, continuous single-frequency light from a tunable DFB BFY diode laser (Toptica DLC PRO) with central wavelength $1550 \mathrm{~nm}$ and linewidth <2 MHz, is injected via the PM fiber array into any desired input channel. In order to monitor the transmission of the processor an array of 8 InGaAs photodiodes (Thorlabs FGA01FC) with FC/PC bulkheads is connected to the 8 output fibers. The signal from each photodiode is collected by a National Instrument BNC-2110 card, readout and recorded with a laptop computer and control software (Labview) via a NI USB-6212 analog-to-digital converter card (see Appendix A).

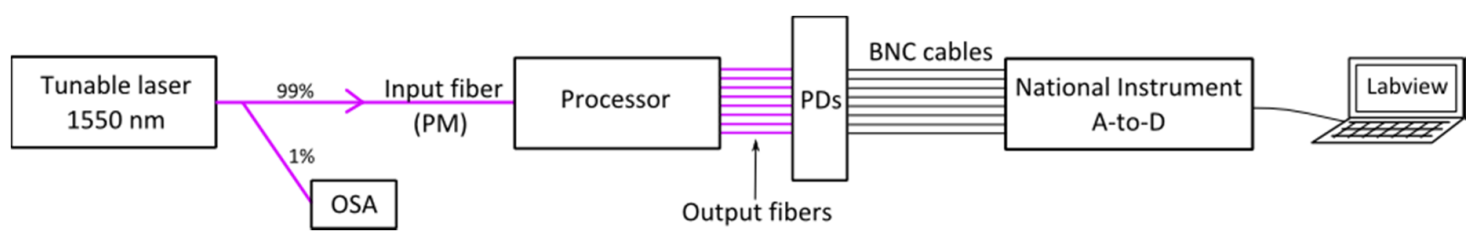

Fig. 4.2 Experimental setup for the characterization of the processor. Single-frequency CW light from a tunable laser set at $1550 \mathrm{~nm}$ is injected into the processor via fiber coupling. $1 \%$ of the output power is used to monitor the injected wavelength. An array of 8 output fibers is connected to an array of photodiodes for detection. The photodiodes signals are read out and acquired via Labview software on a laptop by connecting the PDs via BNC cables to a National Instrument analog-to-digital (A-to-D) converter system.

\subsection{Optical characterization}

As described in Section 2.2.5 characterizing a multi-stage architecture means measuring the response of each thermo-optic phase shifter, i.e., the transmitted power of the MZI containing each phase shifter versus the thermally induced phase delay (which is proportional to the square of the applied heater voltage, see Section 2.1.1).

In the following, we report the results on the optical response of the tunable elements of the processor and further properties, e.g., thermal cross-talk, reproducibility, losses and dispersion. 


\subsubsection{Characterization of the tunable beam splitters}

Following the approach introduced in Section 2.2.5, we start the characterization with the first accessible element at the bottom corner of the processor, which is the tunable beam splitter (TBS) labelled B4R6 in Fig. 4.3. These labels are the specific nomenclature of the thermo-optic phase shifters as implemented in the controlling software. Beginning the characterization with B4R6 is chosen because there is direct access to and from this optical element via the input mode 8 and the output mode 8 .

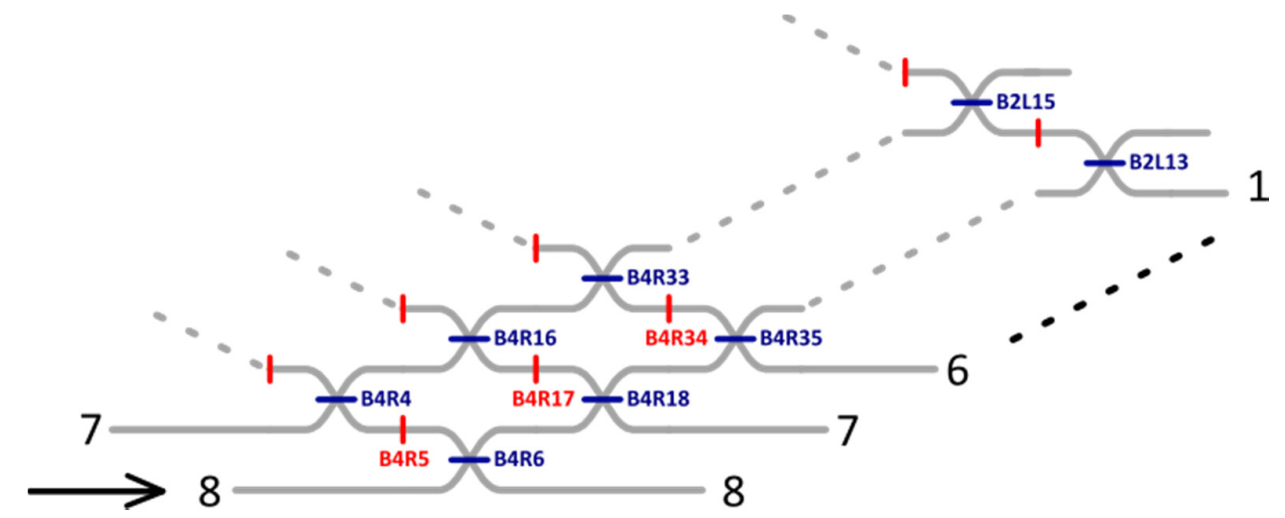

Fig. 4.3 Zoomed schematic of the processor with the specific nomenclature of the thermo-optic phase shifters as given by the controlling software used. Input (output) modes are on the left (right).

We inject light in input 8 and record the transmitted power at all outputs versus $\theta_{\mathrm{B} 4 \mathrm{R} 6}$, i.e., the voltage $\mathrm{V}_{\mathrm{B} 4 \mathrm{R} 6}$ applied to the respective heater. We minimize the transmitted power to the outputs unrelated to the MZI under investigation, i.e., output 6 to 1 , by tuning B4R18 to full reflection at output 7 . In this way the transmission of B4R6 can be determined by the sum of the power at output 8 and 7, minimizing the uncertainty in the transmission function introduced by possible imperfections of unrelated BSs.

The data points in Fig. 4.4 show the transmission values recorded by tuning B4R6. We observe, following the expected sinusoidal variation, that above a certain heater power (expressed as square of the applied voltage), at $\mathrm{V}_{\max }{ }^{2}=10.24 \mathrm{~V}^{2}$, the transmitted power remains constant. This means that the maximum attainable phase shift of that heater is reached (dashed line). This limitation is due to the heater control electronics set to this value for safety reason, i.e., for avoiding permanent damage by a too high temperature. The sinusoidal functions next to the data points is a fit by the theoretically expected tuning (Eq. 1.16) in which the data points above $V_{\max }$ are not considered for fitting (circles). It can be seen that the experimental data are represented by the fit 
curve with high fidelity, i.e., the mutual deviation in transmission is typically less than about $1 \%$.

A somewhat stronger deviation regards the visibility, i.e., the difference between maximum and minimum transmission. We notice that the visibility of the sinusoid is

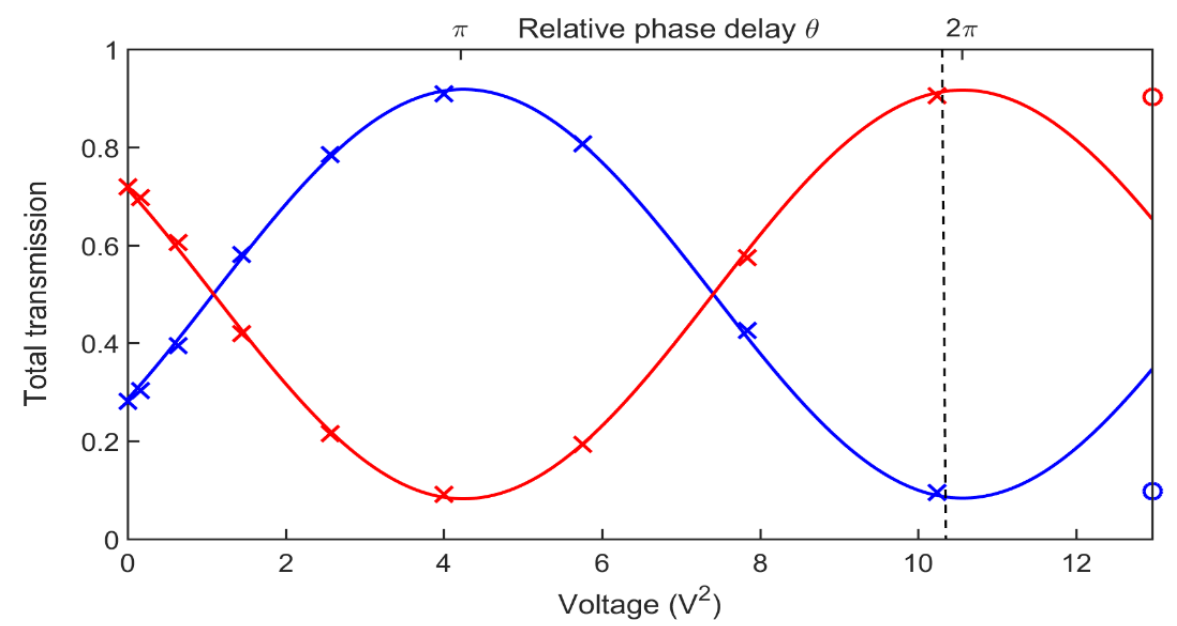

Fig. 4.4 Total transmission of the first accessible TBS, i.e., B4R6. The experimental data (cross) for output 7 (red) and 8 (blue) are fitted by the sinusoidal function from Eq. 1.16 in Chapter 2 and, in this graph, are already normalized by the incoupling and outcoupling efficiencies obtained as described in section 4.3.5 in this Chapter.

lower than the ideal value of unity, which means that one or both of the two coupler values in B4R6 deviates from its ideal value of 0.5 (section 2.2.4). Finally, it can be seen that B4R6 cannot be tuned over a full period $(2 \pi$ interval of phase at the upper horizontal axis), due to the named voltage safety limit, and that there is a non-zero phase at zero heater voltage (offset phase). All these deviations can be addressed to fabrication imperfections as described in section (2.2.4).

For calibrating the heater phase dependence of the transmission fit curve vs the applied voltage, we express the heater phase as $\theta=c+d \cdot V^{2}$ (Sect. 2.1.1), where the first constant, $c$, is the offset phase at zero voltage. The second constant, $d$, is chosen such that the transmitted power to output 8 is maximum at the voltage belonging to the first maximum of the fit curve. Finding the values for $\mathrm{c}$ and $\mathrm{d}$ via a sinusoidal fit as described above and having recorded the minimum and maximum transmission values concludes the characterization and calibration of the first TBS. Hereafter, the transmission of B4R6 can be set to all desired transmission values within the visibility range and safety range, by applying an according heater voltage.

The characterization can then proceed with the second TBS, B4R18, by setting B4R6 to cross (see inset Fig. 2.7). As before, we minimize the transmitted power at unrelated outputs, which are now the outputs 1 to 5 and output 8 , by tuning the following TBS B4R35. The voltage at which the power at output 8 is minimized is already known from the previous characterization step for B4R6. From the transmission at outputs 7 
and 6 versus the tuning of the corresponding thermo-optic phase shifter, i.e., $\theta_{\mathrm{B} 4 \mathrm{R} 18}$, we determine again the accessible phase tuning range, the coupling coefficient of the directional couplers and the voltages corresponding to bar, cross, and 50:50 settings (via finding the respective calibration constants $\mathrm{c}$ and $\mathrm{d}$ in Eq. 1.16).

Following the described procedure repeatedly, we characterize all the TBSs of the first diagonal up to B2L13. After B2L13, we send the diode laser light into the next input (input 7) and characterize all the TBSs in the second diagonal (from B4R4 to B2L15). We complete the characterization of all the TBSs by injecting light into all of the remaining input of the processor (6 to 1). During the entire TBS characterization procedure, all phase shifters (PS) between the TBSs remain turned off, i.e., no voltage is applied.

Summarizing the results of the tunable beam splitter characterization, we find that the average power coupling ratio, $\overline{\mathrm{k}}_{\mathrm{BS}}$, of the 128 directional couplers (two couplers per TBS $\times 64$ unit cells) is very close to the ideal value of $\mathrm{k}_{B S}=0.5$. The average measured value amounts to $\overline{\mathrm{k}}_{\mathrm{BS}}=0.497$ with a standard deviation of $\sigma_{\mathrm{BS}}=0.126$, obtained by fitting the experimental transmission spectra as in Fig. 4 with Eq. 1.16. The average phase range over which the 64 TBSs can be tuned is $\bar{\theta}_{\mathrm{BS}}=4.59 \mathrm{rad}\left(\approx \frac{3}{2} \pi\right)$, and the average range in splitting ratio is adjustable between $5 \%$ and $95 \%$ (see Fig. 4.5 for an overview).

In order to extend the phase range to a full period, $\mathrm{V}_{\max }$ may be increased in the electronic control, as described in Section 2.2.4. However, in order to not risk damage with the current settings and according phase range limit, some of the main phase settings in some of the TBSs, bar or cross, remain inaccessible. We find that about $20 \%$ of the TBSs cannot be set to either bar or cross phase, but that the accessible phase remains an average value of $0.35 \mathrm{rad}$ or $0.2 \mathrm{rad}$ away from the desired values, respectively.

Another consideration is required regarding ambiguity of phase settings. The 50:50 setting occurs, ideally, at two different phase settings, i.e., $\frac{\pi}{2}$ and $\frac{3 \pi}{2}$. In our processor, only one of the two phases is always accessible, depending on whether the maximum transmission appears after (A) or before (B) the transmission minimum (Fig. 2.10 Section 2.2.4). In case $A$ we assign $\theta=\frac{\pi}{2}$ to the $50: 50$ setting, while in case $B$ we assign $\theta=\frac{3 \pi}{2}$. During characterization we find only one TBS whose tunability is very narrow, i.e., less than $\pi$. 

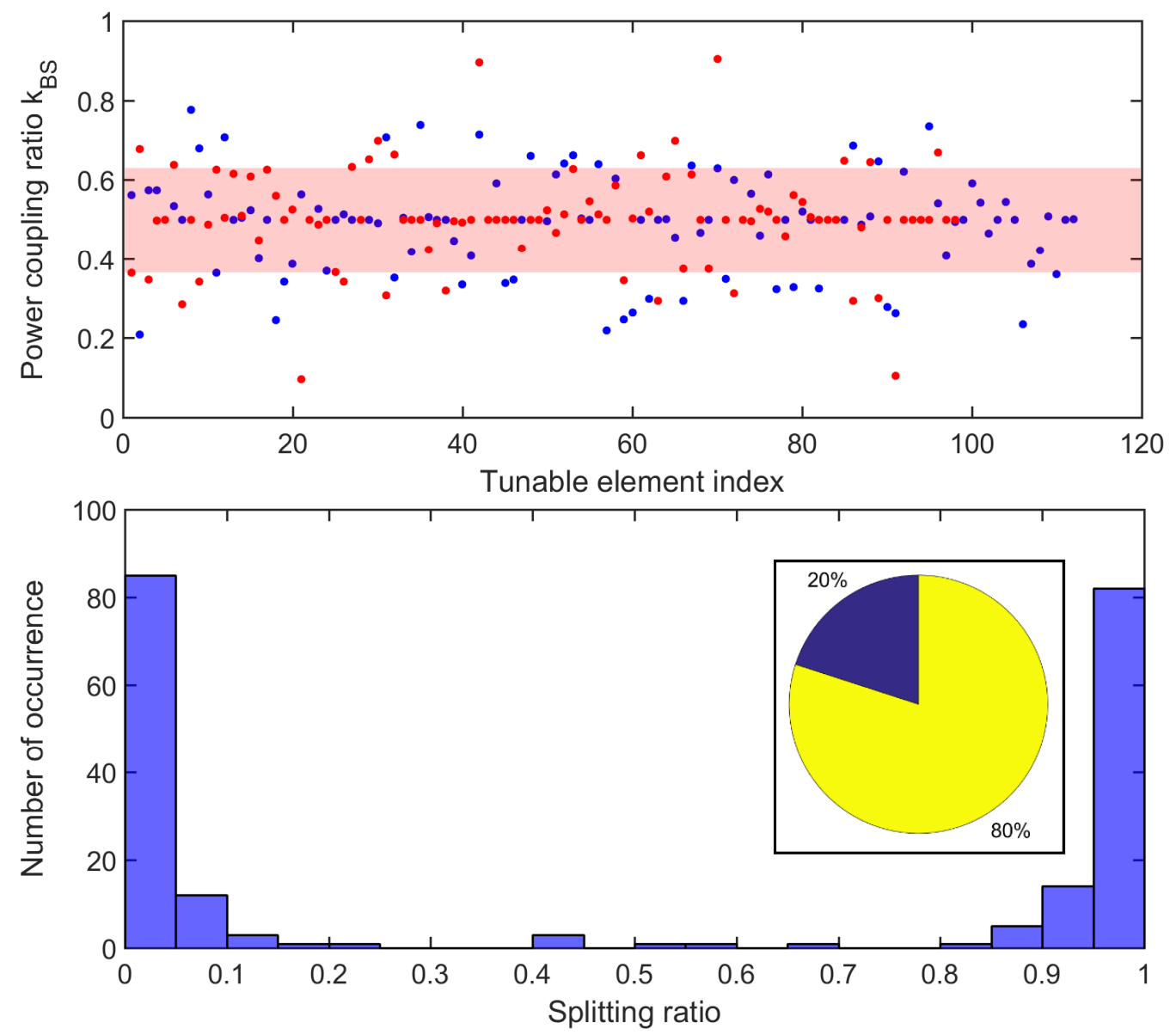

Fig. 4.5 Characterization results summary. (top) Power coupling ratio distributions for all the directional couplers of the processor obtained from the transmission curves of the TBSs (blue) and PSs (red). The width of range shown in red is $2 \sigma$, centered around the mean value which is very close to 0.5 . (bottom) Overview of the splitting ratio, i.e., maximum and minimum transmission, for all the tunable elements. (inset) $20 \%$ of the tunable element cannot reach either the cross or bar setting.

\subsubsection{Characterization of the phase shifters}

Once all the TBSs are characterized, the response of the phase shifters (PS) can be characterized and calibrated as follows, see also the procedure introduced in Sect. 2.2.5.

We start with B4R5, by injecting light into input 7 and by measuring the transmitted power at output 7 and 6 versus $\varphi_{\mathrm{B} 4 \mathrm{R} 5}$ (Fig. 4.3). We set the surrounding tuning elements such as to form a MZI where the 50:50 directional couplers are implemented as two TBSs, set to their respective 50:50 settings. Specifically we set both B4R4 and B4R18 to 50:50, while we set B4R16, B4R6 and B4R35 to bar. We obtain a response similar to the one described above (Fig. 4.4). 
Once characterized via according calibration constants $\mathrm{c}$ and $\mathrm{d}$, we set B4R5 to cross, in order to balance the path lengths from B4R4 to B4R18 via B4R6 and B4R16. This setting allows to characterize also B4R17. In this case one expects an ideal response (i.e., showing zero offset), as the two paths are balanced already by having set B4R5 to cross before. This is indeed what we observe.

Next, we set also B4R17 to cross for characterizing the following phase shifter B4R34. In order to do so, we form again a MZI around it, i.e., by setting B4R4 to cross, B4R16 and B4R35 to 50:50 and B4R18 and B4R33 to bar. Due to fabrication imperfections, however, there will be spurious light entering the interferometer from undesired channels, e.g., from the back of B4R18 (not being perfectly bar) because also B4R4 is not perfectly cross, affecting thus the overall response of the interferometer. To minimize the chance of spurious light we set all elements around the MZI to full transmission, e.g., setting B4R6 to cross. However, as also surrounding elements can be imperfects, spurious light is still present and interference in the MZI will now involve at least three light waves as depicted in Fig. 4.6. To analyze the situation, we describe the interference in terms of the three light fields of amplitudes $\left|E_{A}\right|,\left|E_{B}\right|,\left|E_{C}\right|$ (dashed, point and dash-point lines in Fig. 4.6) versus each of the phase shifters $(\mathrm{A}=$ B4R34, B = B4R17, C = B4R5). In this, we take into account that a light field crossing a TBS leaves that TBS with a $-\frac{\pi}{2}$-phase:

$$
\begin{aligned}
& \mathrm{I}^{\mathrm{A}}=\left(\mathrm{I}_{\mathrm{ABC}}+2 \mathrm{E}_{\mathrm{BC}}\right)+2\left(\mathrm{E}_{\mathrm{AC}}+\mathrm{E}_{\mathrm{AB}}\right) \cdot \cos \varphi_{\mathrm{AB}} \\
& \mathrm{I}^{\mathrm{B}}=\left(\mathrm{I}_{\mathrm{ABC}}-2 \mathrm{E}_{\mathrm{AC}}\right)+2 \mathrm{E}_{\mathrm{BC}} \cdot \cos \varphi_{\mathrm{BC}}+\mathrm{E}_{\mathrm{AB}} \cdot \cos \varphi_{\mathrm{AB}} \\
& \mathrm{I}^{\mathrm{C}}=\left(\mathrm{I}_{\mathrm{ABC}}-2 \mathrm{E}_{\mathrm{AB}}\right)+2 \mathrm{E}_{\mathrm{AC}} \cdot \cos \varphi_{\mathrm{AC}}+2 \mathrm{E}_{\mathrm{BC}} \cdot \cos \varphi_{\mathrm{BC}}
\end{aligned}
$$

where $\mathrm{I}^{\mathrm{i}}$ is the intensity at the output when tuning phase shifter $\mathrm{i}, \mathrm{I}_{\mathrm{ABC}}=\mathrm{I}_{\mathrm{A}}+\mathrm{I}_{\mathrm{B}}+\mathrm{I}_{\mathrm{C}}$ with $\mathrm{I}_{\mathrm{A}}=\left|\mathrm{E}_{\mathrm{A}}\right|\left|\mathrm{E}_{\mathrm{A}}\right|$ and, e.g., $\mathrm{E}_{\mathrm{AB}}=\left|\mathrm{E}_{\mathrm{A}}\right|\left|\mathrm{E}_{\mathrm{B}}\right|$. We see that only $\mathrm{I}^{\mathrm{A}}$ contains solely the phase

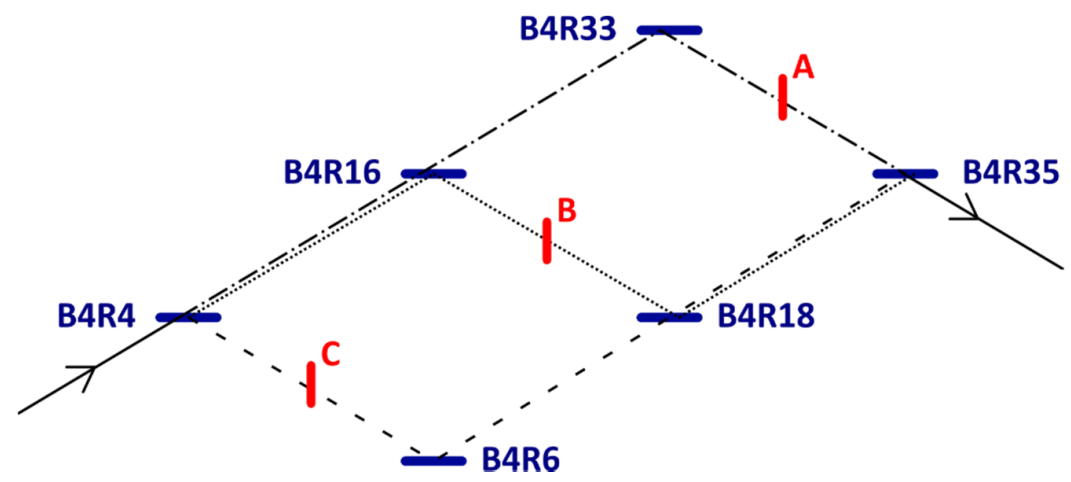

Fig. 4.6 Schematic of a three-waves interference on the processor due to spurious light.

that we are interested in, as an interference term $\cos \varphi_{\mathrm{AB}}=\cos \varphi_{\mathrm{B} 4 \mathrm{R} 34}$, without any 


\section{A reconfigurable linear optical circuit}

contribution from spurious light and thus other phases. This however assumes that the paths $C$ and $B$ are balanced, i.e., setting phase shifters $B$ and $C$ to cross, which is done by the previous step in characterization. We use this same approach to characterize all the PS of the processor.

There is another point in characterization of the phase shifters regarding the phase ambiguity of the TBSs. More specifically, the response of a MZI realized with two ambiguous TBSs depends on whether they are operated at the same phase settings, i.e., both $50: 50\left(\frac{\pi}{2}\right)$ or $50: 50\left(\frac{3 \pi}{2}\right)$, or at different settings. In case the phase settings of the TBS differ by $\pi$, e.g., B4R4 at 50:50 $\left(\frac{\pi}{2}\right)$ and B4R18 at 50:50 $\left(\frac{3 \pi}{2}\right)$ and viceversa, the transmission functions as seen in Fig. 4.4 become phase shifted by $\pi$ (become inverted). The maximum reflection (bar) that is usually assigned to a phase shift of $\pi$ is then found for a phase shift of zero.

Taking into account all of the above and summarizing, we have characterized the optical response of all the 56 accessible PSs in the processor, finding that the average phase tuning range amounts to $\bar{\varphi}_{\mathrm{BS}}=5.07 \mathrm{rad}(\approx 1.6 \pi)$. From the transmission we determine the average value of the power coupling ratio coefficients of the TBSs used to implement MZIs surrounding the PSs, finding $\overline{\mathrm{k}}_{\mathrm{PS}}=0.505$ with $\sigma_{\mathrm{PS}}=0.141$. Again, about $20 \%$ of the PSs do not reach either a bar or a cross setting, but remain at an average deviation of $0.22 \mathrm{rad}$ (0.33 rad) from these (see overview in Fig. 4.5). During characterization we find only one PS whose tunability is very narrow, i.e., less than $\pi$.

\subsubsection{Thermal crosstalk}

All photonic processors need to be characterized with regard to the so-called cross talk, i.e., to the extent that acting on one of the tunable elements slightly changes, undesirably, the setting of other tunable elements. The physical effect responsible for crosstalk is usually the same as the effect employed for the desired tuning.

In our processor, tuning is based on temperature changes in the optical waveguides making use of heat conduction between electric heaters and the underlying waveguide mode volume. The total amount of heat power that can be deployed to the processor is appreciable, in the order of $70 \mathrm{~W}$, because 120 thermooptic phase shifters can be operated simultaneously, with each heater being capable of delivering an average maximum of $600 \mathrm{~mW}$ power. To direct this heat to flow mainly from the heaters vertically through the chip, and not sideways to neighboring heaters and the underlying waveguides, the processor is mounted on a Peltier element heat sink. The Peltier element avoids overheating but is also actively controlled for 
temperature stabilization. The main heat flow is indeed directed vertically from the single heaters into the Peltier element through the waveguide to be controlled. The direction of the heat flow is due to the rather small thickness of the cladding-core region $(16 \mu \mathrm{m})$, the comparatively far distance to neighboring waveguides $(>100 \mu \mathrm{m})$, and the relatively high heat conductivity of the silicon carrier chip. Nevertheless, although globally temperature stabilized, there might be local heat transfer causing thermal crosstalk between neighboring tuning elements. It is thus important to characterize the strength of this crosstalk because the according phase shifts can degrading the targeted functionality of the processor.

In the following, we chose the central part of the processor for the investigation, because there the amount of local temperature buildup is expected to have its maximum effect, i.e., is bigger than, e.g., at the edges of the processor. To perform a measurement in the form of a representative example, we measure how the transmitted optical power of B2R40, in the center of Fig. 4.7, changes with the total number of surrounding heaters turned on to a typical heat power of $600 \mathrm{~mW}$. Light is injected and monitored at input 5 and output 5, respectively, and B2R40 is set to an arbitrary setting which yields, in the shown example, a reference transmitted power

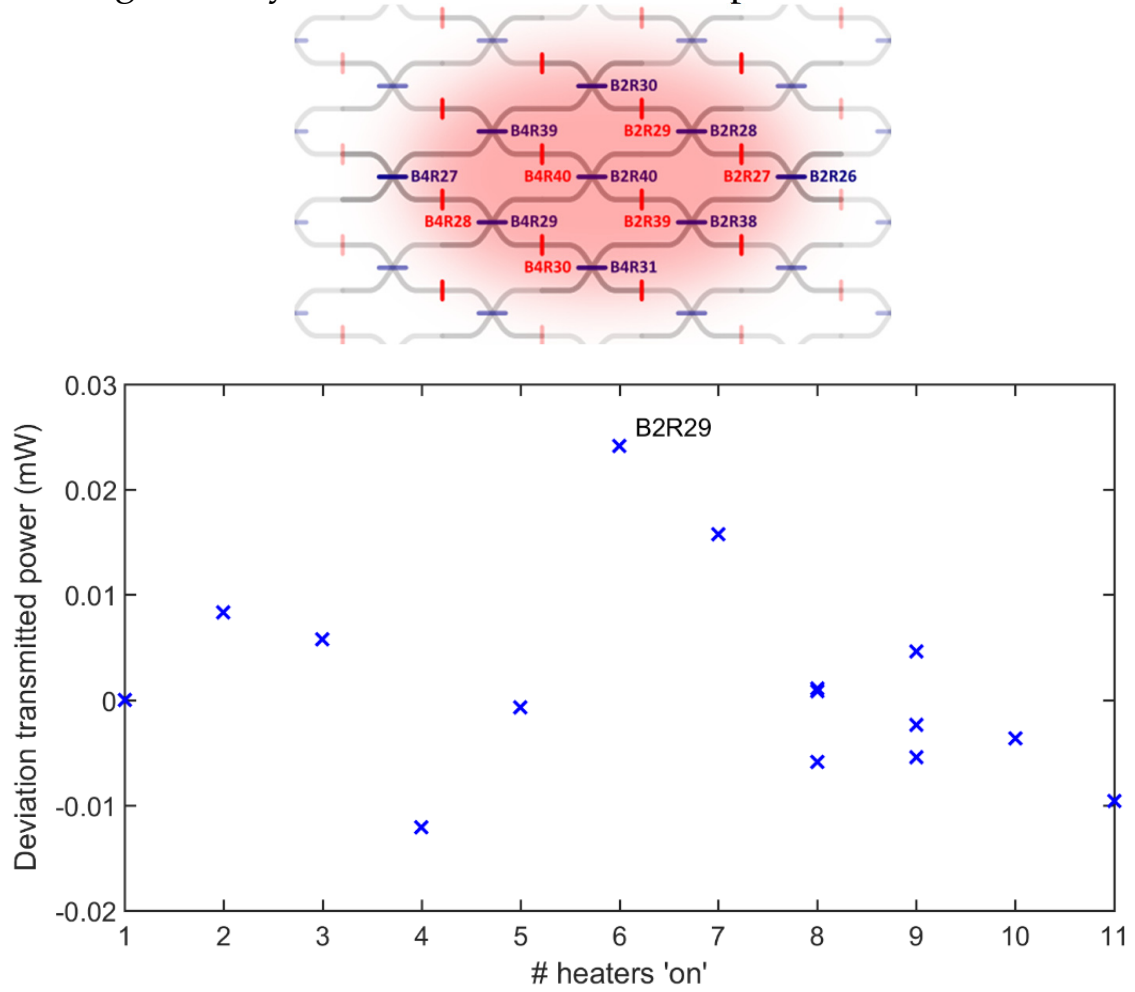

Fig. 4.7 (top) Schematic of the center of the processor where thermal crosstalk is investigated (bottom) Variation of the transmitted power at the bottom output of B2R40 versus the total number of surrounding tuning elements turned 'on'. The greatest influence on the transmitted power is found when turning on of B2R29, which is probably due to sharing of common ground lead. 


\section{A reconfigurable linear optical circuit}

of $\mathrm{P}_{\text {ref }}=0.67 \mathrm{~mW}$ if all the surrounding heaters are turned off. The lower part of Fig. 7 shows that the average of the transmitted power, $\mathrm{P}_{\mathrm{T}}$, deviates by $\overline{\Delta \mathrm{P}}=\overline{\mathrm{P}_{\mathrm{T}}-\mathrm{P}_{\text {ref }}}=$ $0.007 \mathrm{~mW}$ from zero, which is the value with no heater turned on (standard deviation $\left.\sigma_{\Delta P}=0.006 \mathrm{~mW}\right)$. We find that the average variation of the transmitted power amounts to typically only $1 \%$ of the reference transmitted power, and in the worst case (corresponding to the tuning of B2R29) is 3.5\%. As B2R29 is not closer to B2R40 than the other heaters, we conclude that the singular, associated maximum $3.5 \%$ deviation is rather caused by their sharing of a common ground lead in connection with a nonzero internal resistance of the responsible heater driver. Regarding thermal mutual crosstalk between neighboring heaters the measurements suggest that this effect is negligible.

\subsubsection{Reproducibility}

The settings of the integrated optical parts of the processor are found to be highly reproducible. We conclude this, for instance, from the circumstance that the processor showed the same response within the experimental error even after having been transported to a distant laboratory by car and ferry. However, to supply external light to the processor and read out the response at detectors, non-integrated optical fiber connections have to be used. These connections might be a potential cause for lack in data reproducibility.

To test the reproducibility regarding this point, we measure the optical response of the processor after plugging and unplugging the input coupling fiber, repeatedly at all processor input ports. For the main experiments, we do not worry about the reproducibility of the output fiber port plugs or the reproducibility of the voltage settings. The former is assured by having eight output fibers constantly connected to eight photodiodes. High voltage reproducibility (at the $\mathrm{mV}$ level) is assured by the electronic control system.

For testing the input fiber connection reproducibility, the thermo-optic phase shifters of the processors are set to implement an arbitrary transformation, yielding for each input a non-uniform distribution of power across the eight outputs, which represents the transmission matrix of the whole processor for that arbitrary setting (see results in Fig. 4.8). We repeat the same measurements three times by plugging and unplugging the input fiber (different colors in Fig. 4.8). The error bars are given by two standard deviations $(\sigma)$ of the transmitted power. We notice that most of the output distributions are highly reproducible within $2 \sigma$, except when using input 7 , which 
shows a less reproducible behavior. For this reason, reconnecting the input fiber from input 7 was avoided as much as possible.
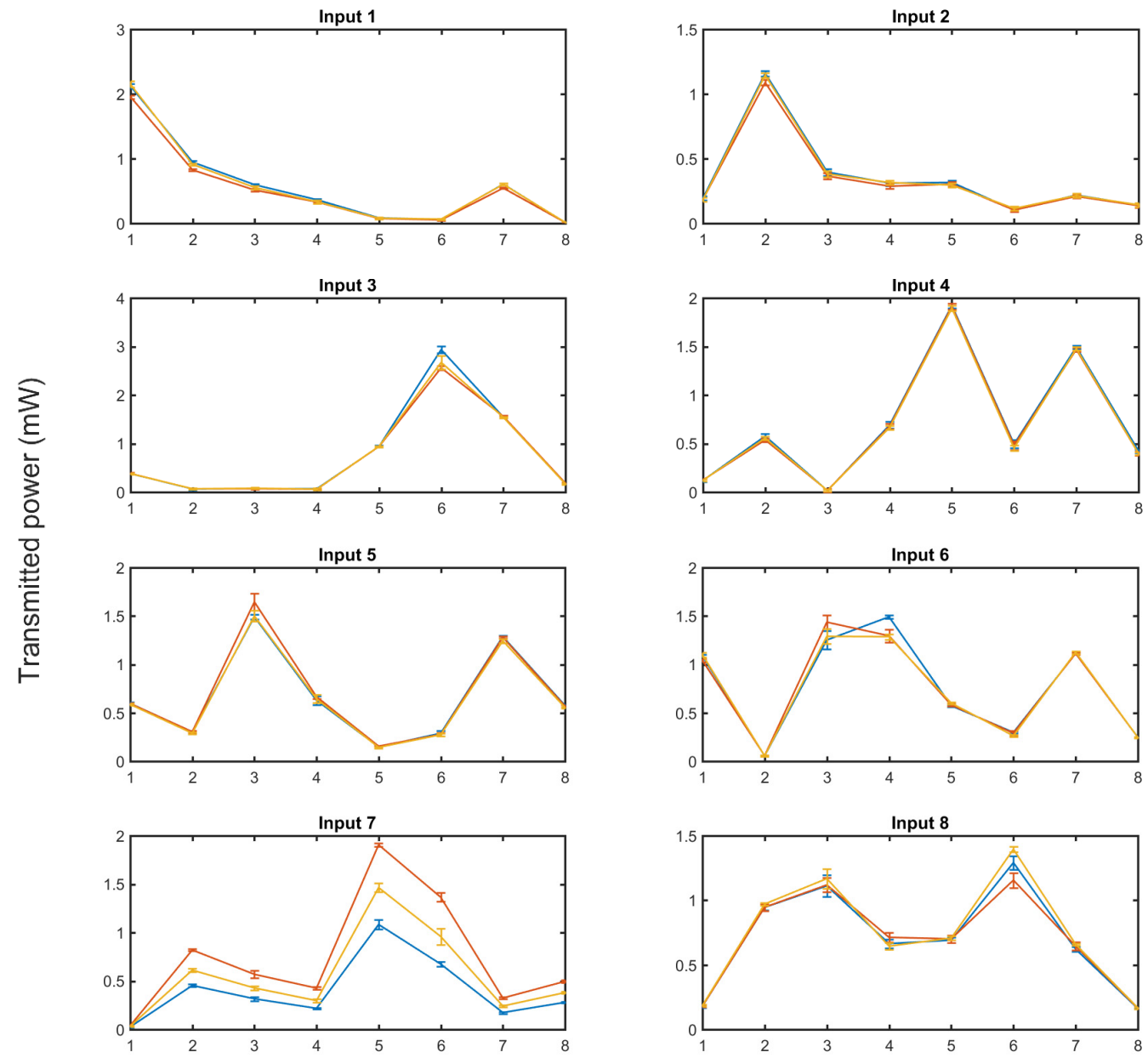

Detector number

Fig. 4.8 Reproducibility of the power distribution for an arbitrary processor setting at the 8 photodiodes. Each color corresponds to a measurement set obtained by unplugging and reconnecting again the input fiber. Error bars are given by the standard deviation of the transmitted power.

\subsubsection{Core response}

In order to determine the transfer matrix associated with the core of the processor, i.e., only the integrated multi-stage MZI interferometer network without connecting fibers, it is required to decouple the response of the thermo-optic phase shifters from other factors such as, e.g., the fiber coupling efficiencies for each mode of the processor. The ability to reconstruct the core response is crucial for the optimization of the response of an imperfect network shown later in Section 4.4. 
The coupling efficiency can be determined by sending light from each input to each output of the processor, assuming that all optical paths from each input to each output are balanced (see Discussions). We measure the transmission matrix $\mathrm{T}$

$$
\mathrm{T}=\left(\begin{array}{ccc}
\mathrm{I}_{1} \mathrm{O}_{1} & \cdots & \mathrm{I}_{1} \mathrm{O}_{8} \\
\vdots & \ddots & \vdots \\
\mathrm{I}_{8} \mathrm{O}_{1} & \cdots & \mathrm{I}_{8} \mathrm{O}_{8}
\end{array}\right)
$$

where $\mathrm{I}_{\mathrm{i}}$ and $\mathrm{O}_{\mathrm{i}}$ are the input and output fiber coupling efficiency values of mode $i$, respectively, with $\mathrm{i}=1, \ldots 8$. To evaluate such measurement, each matrix element $\mathrm{I}_{\mathrm{i}} \mathrm{O}_{\mathrm{j}}$ should actually have been written to contain also a factor $\mathrm{L}_{\mathrm{ij}}<1$ that represents the on-chip propagation loss for each path from input mode i to output mode $\mathrm{j}$. However, since we consider here approximately balanced optical paths, $\mathrm{L}_{\mathrm{ij}}$ becomes a common multiplicative factor, $\mathrm{L}$, outside the matrix and is thus not relevant for the retrieval of the fiber coupling efficiencies.

In order to obtain the fiber coupling efficiencies at each input and output waveguide of the processor, we perform a singular value decomposition (SVD) [14] of the measured matrix T. The coupling efficiency matrix is then used as normalization for all subsequent measurements in Section 4.4, such to access the core response of the processor and thus reconstruct its transfer matrix. Using singular value decomposition, $\mathrm{T}$ is factorized into three matrices $\mathrm{U}, \mathrm{S}, \mathrm{V}$ where $\mathrm{U}$ and $\mathrm{V}$ are unitary matrices and $S$ is a diagonal matrix containing the so-called singular values of the decomposition in descending order from the top left element. The column and row of $\mathrm{U}$ and $\mathrm{V}$, respectively, corresponding to the largest singular value of $\mathrm{S}$ are the vectors that, if multiplied, will give the best approximation of $\mathrm{T}$, i.e.,

$$
\begin{aligned}
& \mathrm{S}(1,1) \gg \mathrm{S}(\mathrm{i}, \mathrm{i}), \quad \mathrm{i}=2, \ldots 8 \\
& \mathrm{~T} \approx \mathrm{Col}_{1}(\mathrm{U}) \cdot \mathrm{S}(1,1) \cdot \operatorname{Row}_{1}(\mathrm{~V})
\end{aligned}
$$

Hence, the first column vector of $U$ and the first row vector of $\mathrm{V}$ correspond to the input and output coupling efficiency $\mathrm{I}_{\mathrm{i}}$ and $\mathrm{O}_{\mathrm{i}}$, respectively.

We reconstruct matrix $\mathrm{T}$ by sending light from each input to each output and measuring the transmitted power at all outputs via the photodiode (PD) array (see Appendix B). After SVD, we retrieve the input and output coupling efficiency vectors $\overrightarrow{\mathrm{I}}$ and $\overrightarrow{\mathrm{O}}$ given by

$$
\begin{aligned}
\overrightarrow{\mathrm{I}} & =(0.29,0.22,0.36,0.41,0.41,0.45,0.26,0.34) \\
\overrightarrow{\mathrm{O}} & =(0.27,0.34,0.34,0.33,0.42,0.46,0.44,0.06)
\end{aligned}
$$


It can be seen that the coupling efficiencies of inputs and outputs have an average value of about $34 \%$, with only output mode $\# 8$ showing very low efficiency (6\%).

\subsubsection{Dispersion and transparency}

Reconfigurable LOCs are attractive for, e.g., quantum information processing and thus might have to be interfaced with single-photon sources that operate at various different wavelength ranges and time structures. Regarding the wavelength range around $1550 \mathrm{~nm}, 780 \mathrm{~nm}$ and 600-900 nm, many different ways of generating singlephotons are available, such as single-photon down-conversion (SPDC) based on KDP crystals and KTP waveguides, or quantum dot emitters [15-18]. Furthermore, SPDC sources and bi-exciton quantum dots emit pairs of photons that are not necessarily spectrally degenerate. It would be useful thus, for quantum information applications, to have a processor that allow the interfacing with different sources.

To investigate the wavelength dependence of the processor, we measure the optical transparency using wavelength tunable input light. For a reconfigurable multistage architecture the strongest wavelength dependence is expected to be caused by the wavelength dependence of the power coupling coefficients, $\mathrm{k}_{\mathrm{i}}$, of the directional couplers. The reason is that the coupling coefficients depend on the spatial overlap of the evanescent fields of the two coupled waveguides. These evanescent field regions, and thus their overlap, becomes smaller with increasing light frequency. As a result one expects that the coupling efficiency of a directional coupler designed for a wavelength of $1.55 \mu \mathrm{m}$, as is here the case, becomes smaller with shorter wavelength.

The according measurements are performed using a spectrally broadband (supercontinuum) light source (Fianium) and a Fourier-Transform Infrared Spectrometer (Bio-Rad), see measurement data in Fig. 4.9.

For providing a first basic test, light is injected into input 8 , where the circuit complexity is smallest with only a single beam splitter (B4R6) between this input and output 8 . The power transmitted to output 8 is recorded by a photodiode for different voltage settings of B4R6. In this measurement, due to decreasing coupling vs decreasing wavelength, most of the injected light should leave at output 8 , increasingly independent of the coupler setting. 


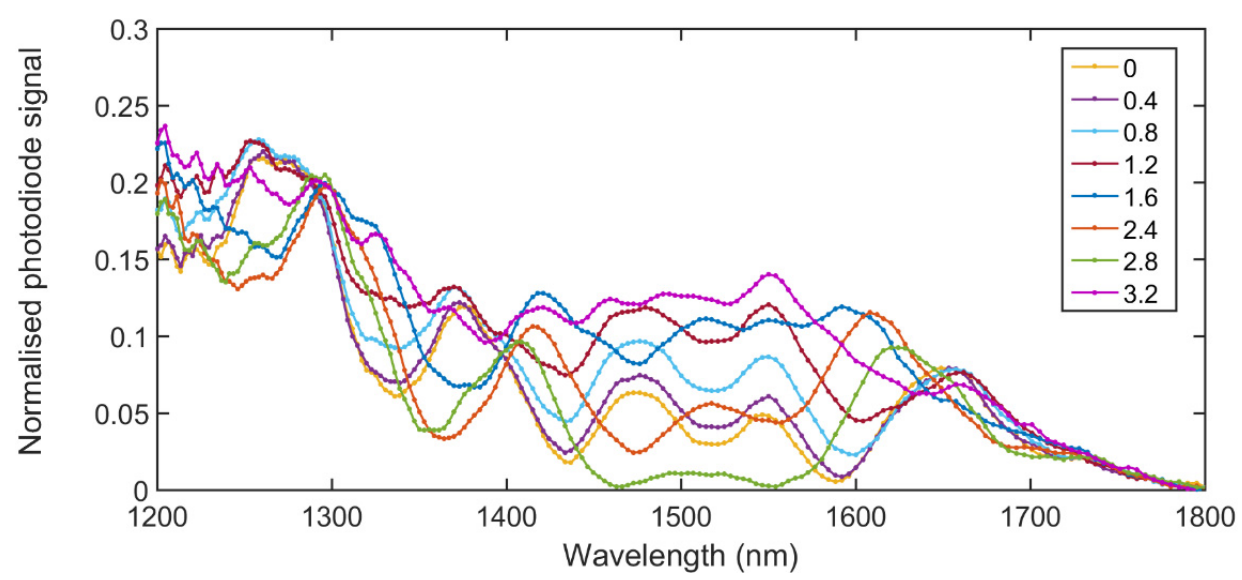

Fig. 4.9 Transmission spectrum of B4R6 for different voltage settings (different colors) over the transparency range of the chip. The signal detected on the photodiode behind the chip is normalized to a reference spectrum of the source light.

Figure 4.9 shows the tunability of the processor over its transparency range via actuation of B4R6. It can be seen that the widest tuning range of the coupler is indeed achieved at long wavelengths, within 1450 and $1600 \mathrm{~nm}$. This is the wavelength range for which the waveguide cross-section has been optimized, as well as the evanescent field overlap and optical length of the directional coupler. Beyond this range, the tunability drastically reduces. One finds occasional wavelengths of medium tunability, e.g., 1360 nm (Fig. 4.10).

Another important observation in Fig. 4.9 is that in a certain range around the central design wavelength (around $1550 \mathrm{~nm}$ with about $10 \mathrm{~nm}$ bandwidth) all of the coupling ratios show only weak variations vs wavelength. This should enable to process ultrashort pulses with ps pulse duration, such as generated by the pulsed

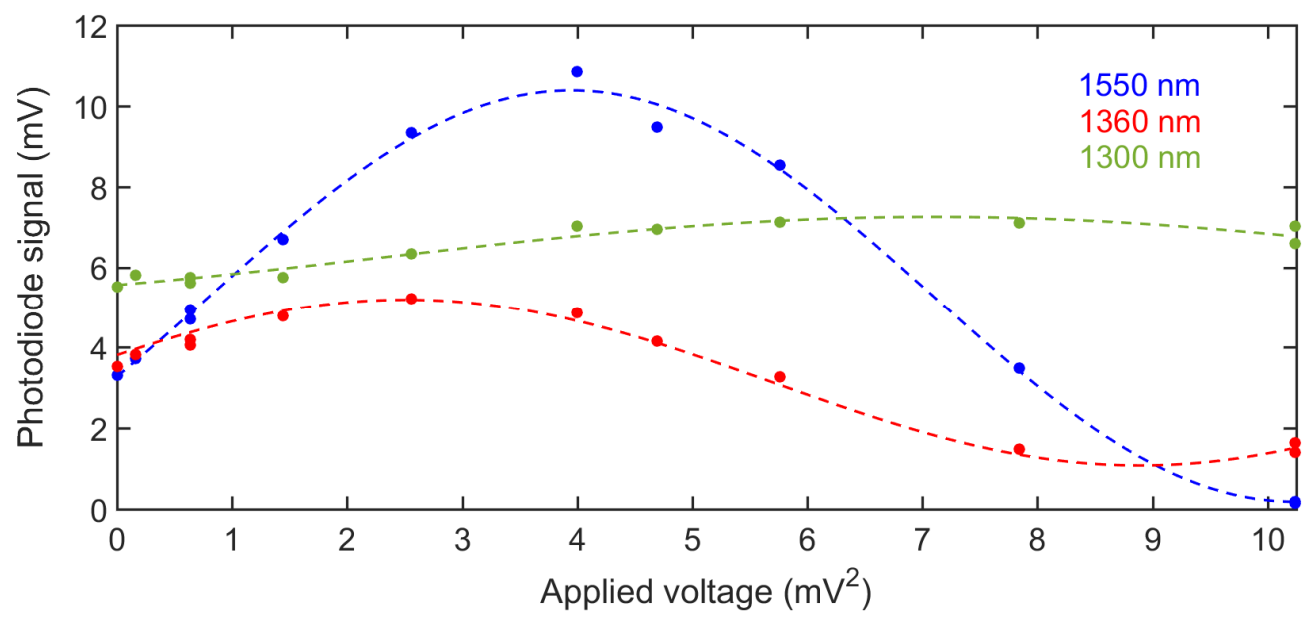

Fig. 4.10 Tunability of B4R6 for three specific wavelengths. 
single photon source (having $3 \mathrm{~nm}$ bandwidth) used for quantum optical experiments described in Chapter 5.

\subsection{Improving the response of an imperfect linear optical circuit}

Many of the applications of reconfigurable LOCs, e.g., boson sampling, rely on the ability of a processor to perform any arbitrary unitary transformation, implying the full reconfigurability of each unit cell. As preparation for configuring the processor and for investigating the range of configurability, the previous section reported the characterization and calibration of the optical response of the individual cells, comprising tunable couplers and phase shifters. We find that, due to fabrication imperfections, there are certain limits regarding the parameter range that can be accessed. Full reconfigurability is given only if all phase shifters are tunable across a range of $2 \pi$. Figure 4.11 shows an overview of the color-coded tuning range of all the 120 characterized phase shifters. It can be seen that most phase shifters allow tuning across only about $3 / 2 \pi$, with two shifters below pi and one close to $2 \pi$. Accordingly, there should be limits or imperfections in the reconfigurability of the processor.

Attempting to solve this problem, in the following we show that, nevertheless, it is possible to improve the response of the processor to a certain degree with

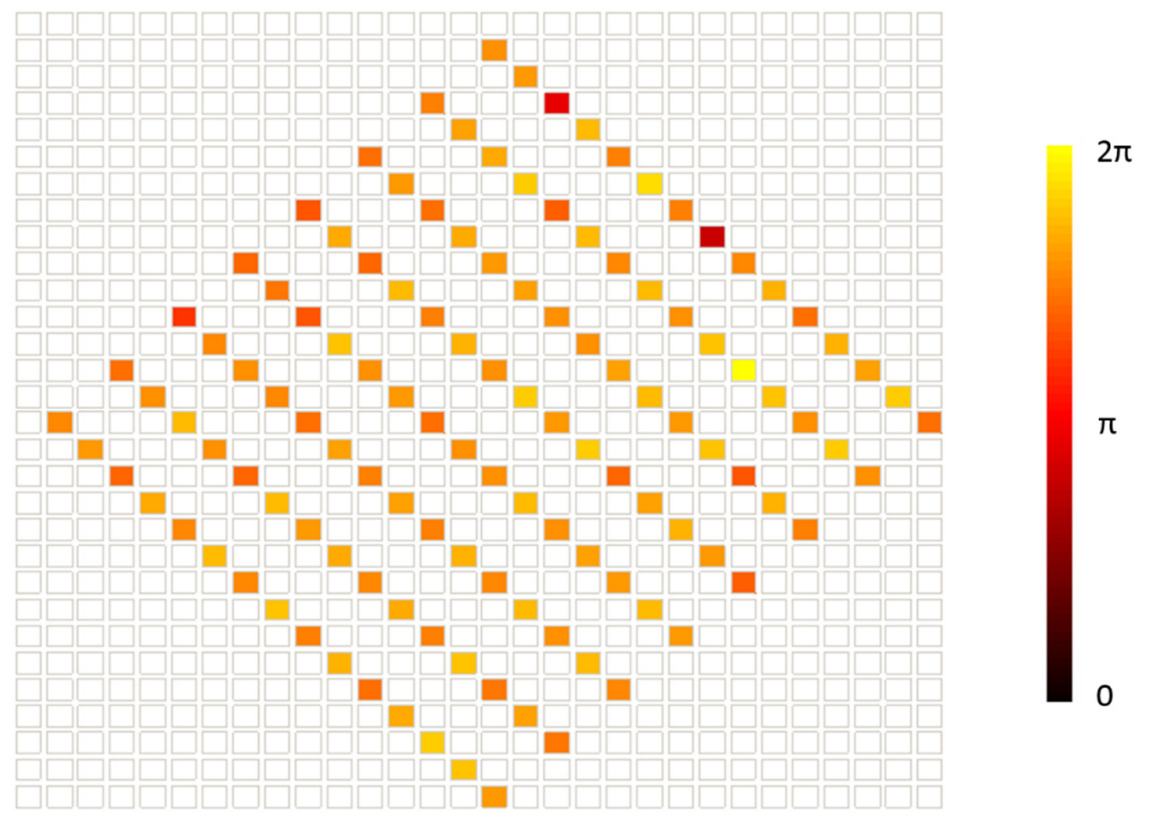

Fig. 4.11 Overview of the tuning range of all the thermo-optic phase shifters (colored boxes). 
appropriately modified settings, obtained from a mathematical optimization algorithm, such that at least certain sections of processor will perform as an ideal one.

\subsubsection{Implementing arbitrary unitary transformations on imperfect linear optical circuit}

For preparing considerations on the performance of the processor and possible optimization despite the fabrication imperfections, we recall the decomposition of an arbitrary unitary transformation. We consider decomposition into a product of bidimensional transformations [12] and the restrictions and conversion that have to be applied in order to implement such arbitrary unitary transformation on a realistic, i.e., imperfectly fabricated, photonic processor.

Matching the optical structure of the processor, let us recall how to represent an arbitrary $\mathrm{n} \times \mathrm{n}$ unitary transformation $\mathrm{U}$, i.e., $\mathrm{U}^{\dagger} \mathrm{U}=\mathrm{U}^{-1} \mathrm{U}=|\mathrm{U}|^{2}=\mathrm{I}$, using a linear optical circuit formed only by tunable beam splitters and phase shifters. As first demonstrated by Reck et al. [13] for a triangular architecture and successively by Clements et al. [12] for a square one, any $n \times n$ unitary transformation $U$ can be decomposed into a specific ordered product of $2 \times 2$ unitary transformations $\mathrm{T}_{\mathrm{m}, \mathrm{n}}(\theta, \varphi)$, each describing the unit cell between input and output mode $m$ and $n$, respectively. $\mathrm{T}_{\mathrm{m}, \mathrm{n}}(\theta, \varphi)$ is thus given by Eq. 1.15 with an additional phase term $\mathrm{e}^{-\mathrm{j} \varphi}$ multiplying the elements of the first column, representing the effect of the phase shifter preceding the tunable coupler for that unit cell.

As an example, we take the following 3-dimensional arbitrary unitary matrix

$$
\mathrm{U}=\left(\begin{array}{ccc}
0.48-0.14 \cdot i & -0.74+0.34 \cdot i & 0.13-0.27 \cdot i \\
0.26-0.25 \cdot i & 0.2-0.46 \cdot i & -0.36-0.7 \cdot i \\
-0.15-0.77 \cdot i & -0.04-0.3 \cdot i & 0.52+0.14 \cdot i
\end{array}\right)
$$

belonging to a Haar random distribution, as the first non-trivial unitary transformation dimension-wise, and attempt its implementation in our processor at the 8, 7,6 input and output modes (see Fig. 4.12). We choose to implement such transformation at the bottom corner of our processor (Fig. 1) because this is the most directly accessible

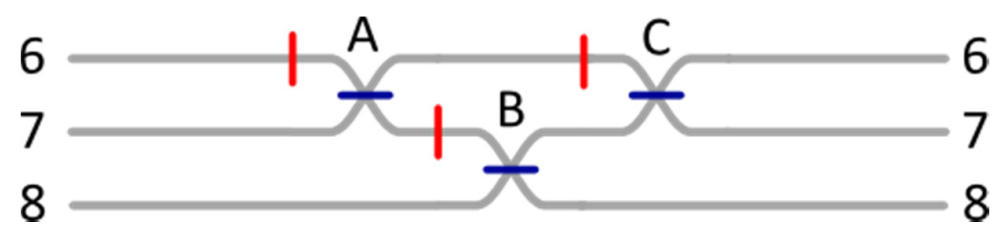

Fig. 4.12 Schematic of the $3 \times 3$ network investigated here as an example, with the alphabetical order corresponding to the matrix multiplication. 
location, not requiring light to go through further unit cells, and this location provides the shortest access of input and output modes 8,7 and 6 to the processor's core. Following the procedure described in $[12,13]$ for the architecture of our processor (Fig. 4.12), we decompose $U$ into the product of three $2 \times 2 \mathrm{~T}_{\mathrm{m}, \mathrm{n}}(\theta, \varphi)$ matrices obtaining $\mathrm{U}_{\mathrm{dec}}=\mathrm{T}_{6,7}(\theta, \varphi)_{\mathrm{A}} \cdot \mathrm{T}_{7,8}(\theta, \varphi)_{\mathrm{B}} \cdot \mathrm{T}_{6,7}(\theta, \varphi)_{\mathrm{C}}$

We notice that the order at which the unit cell matrices are multiplied is the order at which light encounters the unit cells travelling from the far most left input to the far most right output. From the decomposition we thus obtain a set of six phases $\theta$ and $\varphi$ in Table 1, that can be inserted into the corresponding $\mathrm{T}_{\mathrm{m}, \mathrm{n}}$ matrix.

\begin{tabular}{|l|l|l|}
\hline Unit cell & \multicolumn{1}{|c|}{$\theta(\mathrm{rad})$} & \multicolumn{1}{|c|}{$\varphi(\mathrm{rad})$} \\
\hline A & 0.73 & 3.19 \\
\hline B & 1.15 & 0.04 \\
\hline C & 2.41 & 2.62 \\
\hline
\end{tabular}

Table 1 Phase settings from the decomposition algorithm to apply to each unit cell of the processor to perform transformation U. $\theta(\varphi)$ are the phases for the TBS (PS) of the unit cell.

To test that we have carried out the decomposition algorithm correctly such that it mirrors our architecture as in Fig. 4.12, we insert $(\theta, \varphi) \mathrm{i}(\mathrm{i}=\mathrm{A}, \mathrm{B}$ and $\mathrm{C})$ settings into $\mathrm{U}_{\mathrm{dec}}$ and we compare the power distribution, i.e., the modulus squared, of the matrix elements of the target unitary $U$ and $U_{\text {dec }}$ (both Fig. 4.13 left). Given the unitarity of such transformation, the power distribution of the matrix elements of $U$ can be seen as the probability distribution of an incoming light in input mode m, e.g., input mode 8, to get distributed over the output modes, e.g., 8, 7 and 6. Calculating the fidelity of

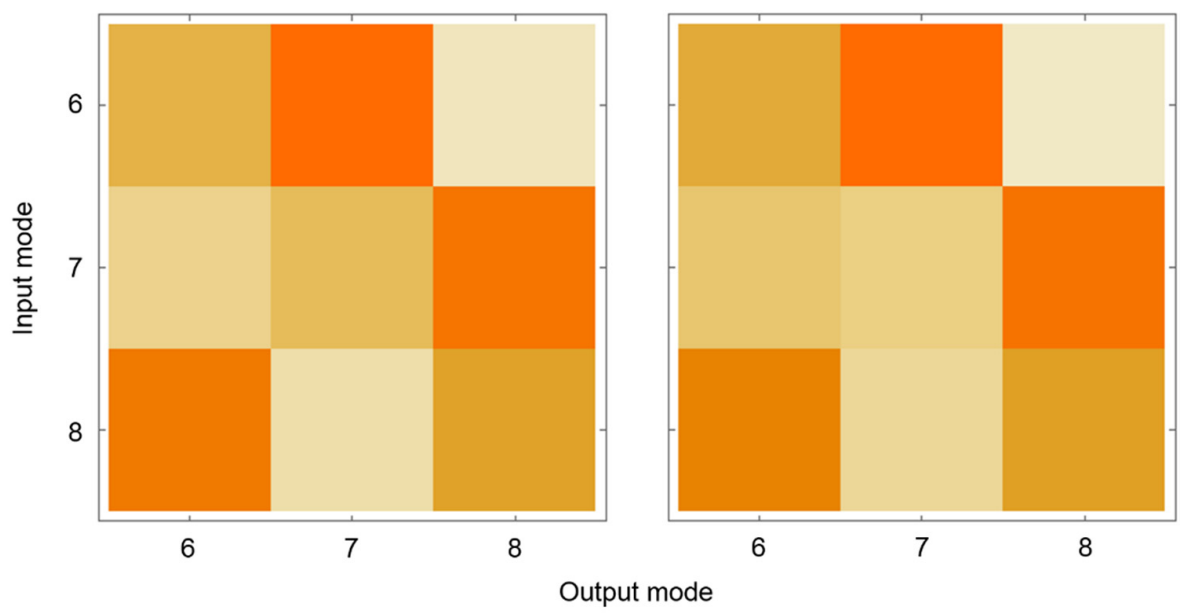

Fig. 4.13 Intensity distribution matrix for $U$, $U_{\text {dec }}$ (both left) and $U_{\exp }$ (right). Mode numbering follow the one in Fig. 4.12. 


\section{A reconfigurable linear optical circuit}

these two probability distributions as described in Chapter 2, we obtain $\mathcal{F}\left(\mathrm{U}, \mathrm{U}_{\mathrm{dec}}\right)=$ 1. This result shows that we implemented the decomposition algorithm correctly.

Based on the described Reck decomposition, and having available the phase tuning range of the phase shifters from characterization, it becomes possible to determine how well our processor can perform any target unitary U. In an ideally fabricated processor, to do so, one would simply set each thermo-optic phase shifter of the processor to the theoretically required corresponding $\theta$ or $\varphi$ (as in Table 1) meaning that for each tunable element the corresponding voltage has to be applied as determined in the characterization and calibration procedure.

However, due to the limitation of the tunable elements it is likely that some of the desired phases $(\theta, \varphi)$ are not achievable. In these cases, we chose the closest achievable phase value $\tilde{\theta}$ and $\widetilde{\varphi}$. Converted into the corresponding voltages obtained by calibration, this is equivalent to choosing the closest available voltage values,

$$
\begin{aligned}
\mathrm{V}_{\mathrm{BS}} & =\sqrt{\left(\tilde{\theta}-\mathrm{c}_{\mathrm{BS}}\right) / \mathrm{d}_{\mathrm{BS}}} \\
\mathrm{V}_{\mathrm{PS}} & =\sqrt{\left(\widetilde{\varphi}-\mathrm{c}_{\mathrm{PS}}\right) / \mathrm{d}_{\mathrm{PS}}}
\end{aligned}
$$

where $\mathrm{c}$ and $\mathrm{d}$ are the fit (calibration) parameters obtained from the characterization of the thermo-optic phase shifters (see Chapter 2.1.1).

Starting with the original target unitary $U$ transformation, after considering the limitation of the tunable elements, the closest voltage settings correspond to a different unitary $\widetilde{U}$ that is theoretically implementable on our processor. To test the quality of the implementation of $\widetilde{U}$ also experimentally, we set each tunable element to $\mathrm{V}_{\mathrm{BS}}$ and $\mathrm{V}_{\mathrm{PS}}$ and measure the power, or probability, distribution matrix $\left|\mathrm{U}_{\text {exp }}\right|^{2}$ at the three outputs of the processor for all the three inputs. Normalizing $\left|U_{\text {exp }}\right|^{2}$ by the coupling efficiency matrix (section 4.3.5) we obtain the intensity distribution of the core of the processor (Fig. 4.13), i.e., the response of only the multi-stage MZI interferometer.

To quantify the similarity between the three unitaries, i.e., $|U|^{2},|\widetilde{U}|^{2}$ and $\left|U_{\text {exp }}\right|^{2}$ we use the respective probability distributions to calculate the mutual fidelities as defined in Section 2.3.1. If the target phases $(\theta, \varphi)$ are within the allowed range of the relative tunable elements we expect $|U|^{2}=|\widetilde{U}|^{2}=\left|U_{\text {exp }}\right|^{2}$, while if $(\theta, \varphi)$ are outside the allowed range $|U|^{2} \neq|\widetilde{U}|^{2}$. For the particular Haar random selected example shown above it turns out that the required (target) phases fall within their accessible ranges. Calculating the fidelities for these transformations we obtain 


$$
\begin{aligned}
& \mathcal{F}\left(|\mathrm{U}|^{2},|\widetilde{\mathrm{U}}|^{2}\right)=1 \\
& \mathcal{F}\left(|\mathrm{U}|^{2},\left|\mathrm{U}_{\text {exp }}\right|^{2}\right)=0.989 \\
& \mathcal{F}\left(|\widetilde{\mathrm{U}}|^{2},\left|\mathrm{U}_{\text {exp }}\right|^{2}\right)=0.989
\end{aligned}
$$

It can be seen that the second and third fidelities are very close to unity, which corresponds to a close-to-ideal implementation of the target unitary, U. However, the remaining deviation from unity, $\mathcal{F}\left(|U|^{2},\left|U_{\exp }\right|^{2}\right)<1$, tells that the experimental intensity distribution is not exactly the expected one. It can also be noted that the fidelity between the calibration-wise achievable unitary $\widetilde{U}$ and the realized one, $U_{\text {exp }}$ is lower than unity as well. This means that measuring the core response was not fully accurate, or that calibrating or setting the phase values was not fully accurate.

So far we evaluated the quality of implementing an arbitrary unitary transformation of the processor's core via a single example of a $3 \times 3$ Haar random unitary. In order to acquire a more general knowledge of how well the processor can perform arbitrary unitary transformations, we repeat the described fidelity measurements with a larger number of examples. We generate 99 different unitary matrices $\mathrm{U}^{\mathrm{i}}$ from the Haar random distribution for different matrix dimensions, i.e., $(3 \times 3),(5 \times 5)$ and $(8 \times 8)$. Each target matrix $\mathrm{U}^{\mathrm{i}}$ is decomposed as a Reck architecture and the corresponding achievable matrix $\widetilde{U}^{i}$ is determined in terms of its voltage settings $V_{B S}$ and $V_{P S}$. The transmitted power of the processor, i.e., $\left|U_{\exp }\right|^{2}$, is then measured and the fidelity of the according probability distribution is calculated.

A first overview regarding the dimension of the target network is shown in the upper part of Fig. 4.14. It can be seen that the number of unitary transformations encountering limitations in their implementation increases with the dimension of the network. The reason is that the number of elements that need to be controlled and the number of phase values that need to be realized per element grows with the dimensions, such that it becomes more likely to find at least one target phase outside the accessible range. Comparison with a fit curve (dashed line) shows that the likeliness increases exponentially with the dimension.

The bottom part of Fig. 4.14 displays the fidelity distribution of the 33 arbitrary unitary transformations per investigated matrix dimension (different colors belong to different dimensions). The measured fidelities are always below unity and it can be seen that the average fidelity (dashed line) decreases with increasing the dimension of the transformation. 

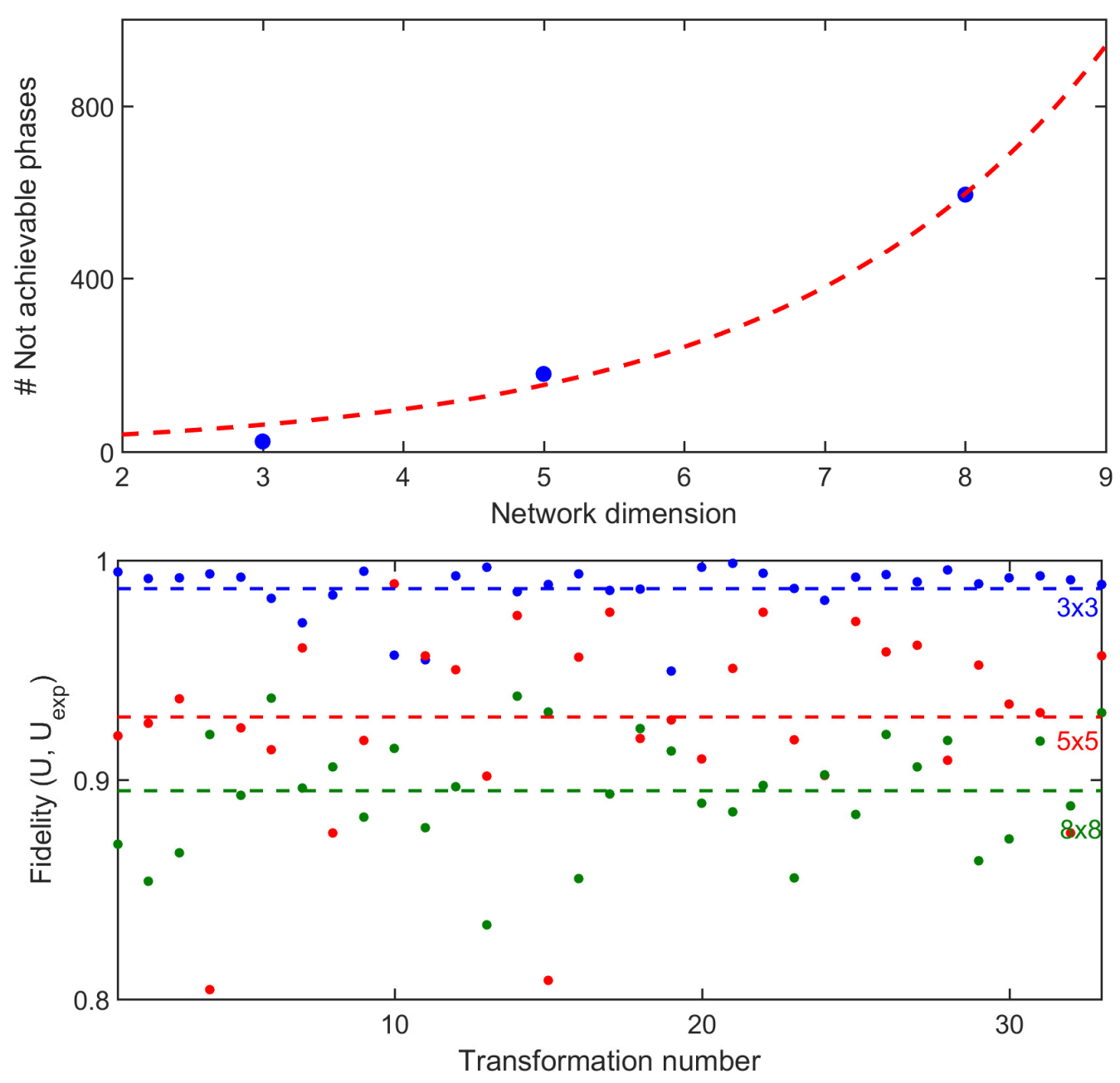

Fig. 4.14 (top) Number of thermo-optic phase shifters encountering a desired phase setting outside their range versus the network dimension. The number of not achievable phases is counted over all the 33 targeted transformation (bottom) Fidelity distribution of the target unitary $U$ and the measured one $U_{\exp }$ for all the transformations (dots). The dashed lines are the fidelity mean values per dimension.

Figure 4.15 shows a comparison between the measured fidelity $\mathcal{F}\left(|\mathrm{U}|^{2},\left|\mathrm{U}_{\text {exp }}\right|^{2}\right)$ and the expected one, i.e., $\mathcal{F}\left(|U|^{2},|\widetilde{U}|^{2}\right)$. One sees that the measured fidelity is always lower than the expected one, and that both the expected and the measured fidelity decreases with increasing the dimension. Firstly, these results confirm our suspicion that the measuring of the core response, the calibration or setting the phase values was not fully accurate. Secondly the results confirm that for larger networks the limitations of the tunable elements decrease the expected fidelity to a larger extent. 


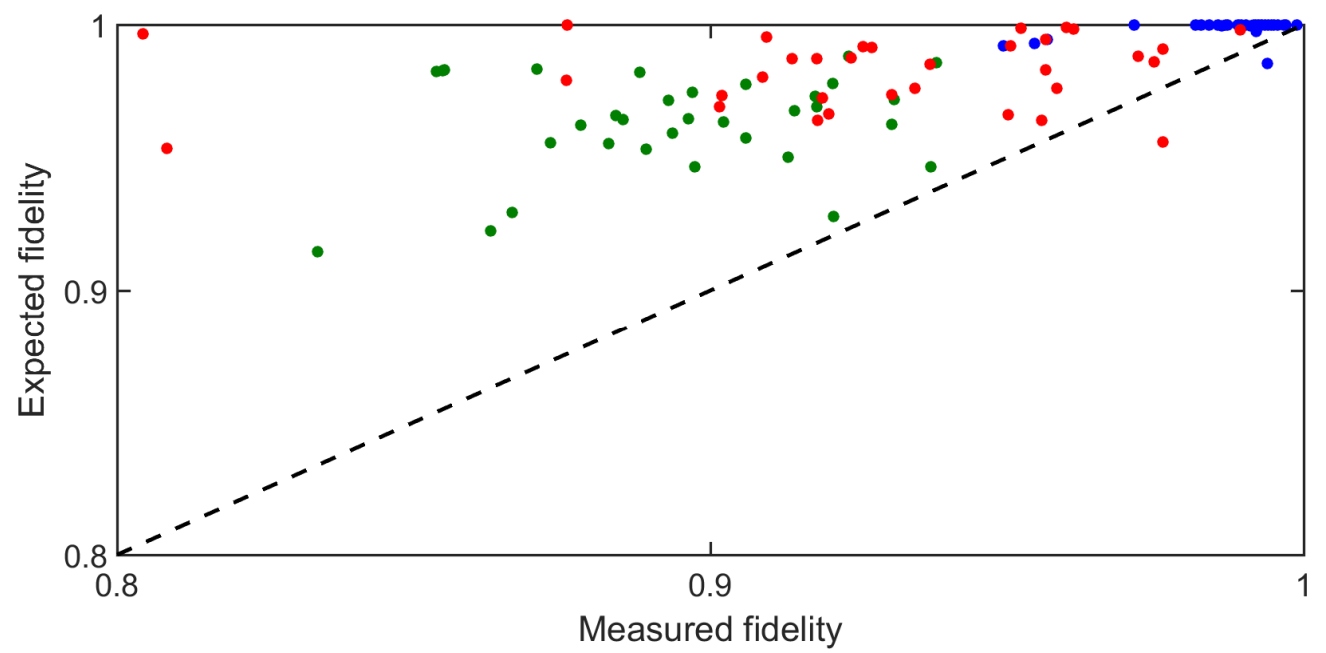

Fig. 4.15 Expected fidelity versus the measured one for network dimension 3 (blue), 5 (red) and 8 (green). The dashed line indicates the identity.

\subsubsection{Overcoming fabrication imperfections with numerical optimization}

In this section we investigate whether it is possible to improve the fidelity of the processor performance in realizing unitary transformations, even if the phase settings for a transformation fall in a range that cannot be accessed. The idea is to vary the phase settings within the accessible range but use different combination of phases in order to increase the fidelity, i.e., to come closer to a target transformation.

This approach resembles training a neural network, as described in the context of a smart spectrometer, where a high-dimensional fit is used to resemble and later recall a complex transmission spectrum. Here a higher-dimensional fit of achievable transformations to a target transformation is used, where the phases are the fit parameters. Minimizing the deviation of the fidelity from unity takes the role of minimizing a nonlinear objective function, in analogy to minimizing the mean-square deviation from the target transformation.

More specifically, within the constraints on the phase range of each tunable element, we search for optimized phase settings that would bring an implementable transformation $\widetilde{U}^{i}$ closer to the targeted one, i.e., to $U^{i}$. In the implementation of the optimization, for each target $(\theta, \varphi)^{\mathrm{i}}$, we look for $(\tilde{\theta}, \widetilde{\varphi})^{\mathrm{i}}$ that minimize the difference between the unity and $\mathcal{F}\left(\left|\mathrm{U}^{\mathrm{i}}\right|^{2},\left|\widetilde{U}^{\mathrm{i}}\right|^{2}\right)$, where now $(\tilde{\theta}, \widetilde{\varphi})^{\mathrm{i}}$ are the optimized phase values and not just simply the closest.

For optimization we define an objective function as $G=1-\mathcal{F}\left(\left|\mathrm{U}^{\mathrm{i}}\right|^{2},\left|\widetilde{\mathrm{U}}^{\mathrm{i}}\right|^{2}\right)$. For each $\mathrm{i}^{\text {th }}$ transformation, a numerical optimization algorithm searches for phase 
settings $(\tilde{\theta}, \widetilde{\varphi})$ that minimize $G$ within the limited range of the corresponding tunable elements. However, minimizing a function defined on a constrained domain is a computationally expensive task. For an alleviation, we convert the problem into an unconstrained one where the domain over which $\mathrm{G}$ is defined is now unbounded but the values that $G$ can assume are limited. In order to do so we search for phases $\left(\tilde{\theta}^{\prime}, \widetilde{\varphi}^{\prime}\right)$ defined as

$$
\begin{aligned}
\widetilde{\theta}^{\prime} & =\left(\tanh \tilde{\theta}-\left(1-\frac{2 \cdot \widetilde{\theta}_{\max }}{\widetilde{\theta}_{\max }-\widetilde{\theta}_{\min }}\right)\right) /\left(\frac{2}{{\widetilde{\tilde{\theta}_{m a x}}}_{\max } \widetilde{\theta}_{\min }}\right), \\
\widetilde{\varphi^{\prime}} & =\left(\tanh \widetilde{\varphi}-\left(1-\frac{2 \cdot \widetilde{\varphi}_{\max }}{\widetilde{\widetilde{\varphi}}_{\max }-\widetilde{\varphi}_{\min }}\right)\right) /\left(\frac{2}{\widetilde{\varphi}_{\max }-\widetilde{\varphi}_{\min }}\right)^{\prime}
\end{aligned}
$$

where $\tilde{\theta}_{\max }\left(\widetilde{\varphi}_{\max }\right)$ and $\tilde{\theta}_{\min }\left(\widetilde{\varphi}_{\min }\right)$ are the maximum and minimum achievable phases of the tunable element belonging to the TBS (PS) of a unit cell. In this way we map the constrained phase domain of each tunable element to the limited values that the hyperbolic tangent of the phase can assume. To more detail, we use the function fminunc in Matlab that finds the minimum of the unconstrained function $G$, by implementing a sequential quadratic programming (SQP) method for optimization [19].

After numerical optimization we convert the optimized phases into voltages and measure the intensity distributions $\left|\widetilde{\mathrm{U}}_{\mathrm{exp}}^{\text {opt }}\right|^{2}$ for all of the 99 samples of Haar random unitary transformations.

In order to analyze the results and successfulness of the optimization, Fig. 4.16 shows the fidelity of each $\widetilde{\mathrm{U}}_{\mathrm{exp}}^{\mathrm{i}}$ after optimization for the three different matrix

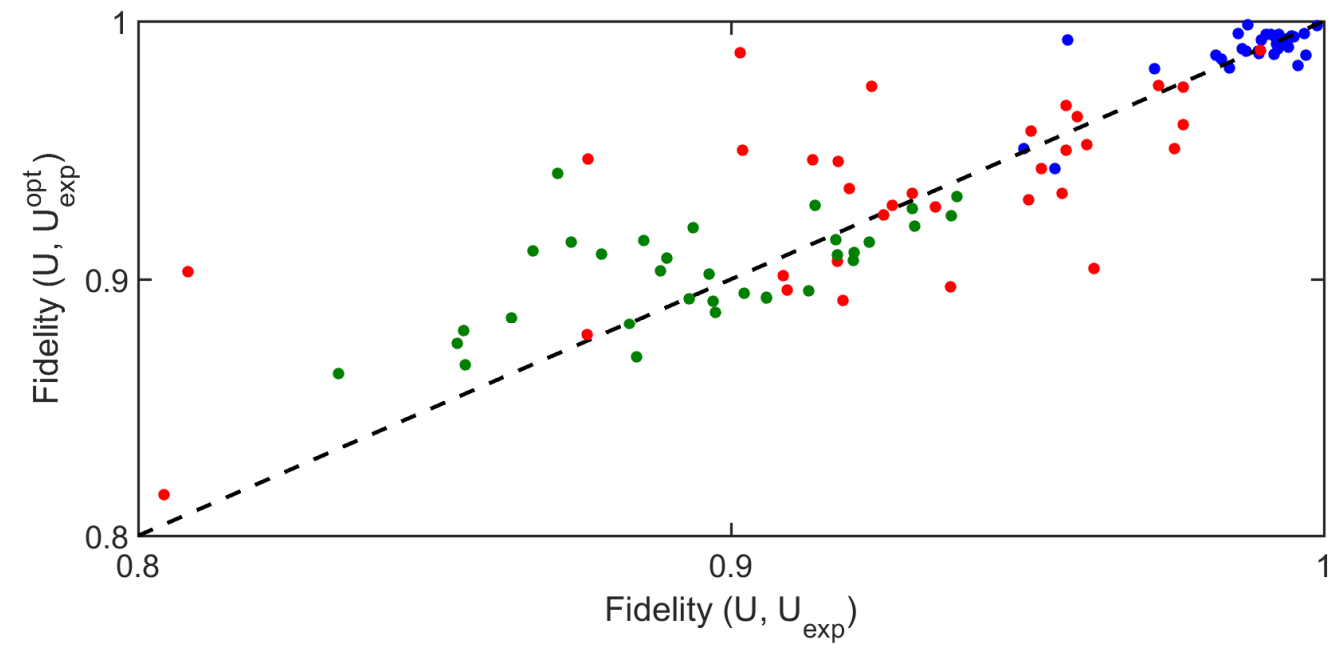

Fig. 4.16 Fidelity improvement for all 33 transformations for network dimension 3 (blue), 5 (red) and 8 (green) (different colors). Dots above (below) the dashed line indicate transformations whose fidelity has been improved (worsened).

dimensions (different colors) versus the fidelity before optimization. We find again that, on average, lower fidelity occurs for a higher matrix dimension, as discussed in 
the previous subsection. On the other hand, transformations with low experimental fidelity are also the ones that are more likely to be improved by the optimization. Transformations that show a high experimental fidelity already before optimization experience a small improvement or even a worsening. The threshold fidelity, below which the transformations get actually improved by the numerical optimization, diminishes with an increasing matrix dimension.

\subsubsection{Discussion}

From the observation that the measured fidelity for each of our 99 samples of Haar random unitary transformations is always lower than the expected one, we conclude that possibly the retrieval of the core response was not fully accurate.

We discuss here a possible explanation of this inaccuracy. In finding the processor's core response we assumed that the optical paths from each input to each output mode are equally long, implying thus that the propagation losses along all possible paths are approximatively the same. Under this assumption the transmission matrix reconstructed in Section 4.3 .5 is given only by the input and output fiber coupling efficiencies.

However, although the processor's core is designed such to assure always constructive interference for recombining signals, the optical paths for signals going from each input to each output mode, thus signals that do not recombine, are not all equally long. Specifically the distance between each unit cell to its two consecutive ones is different depending on the output mode, and the waveguides connecting the processor's core to the chip facets do not fully compensate for these path differences. For example, the optical path that goes from input mode 8 to output mode 8 is much shorter than the optical path that goes from input mode 1 to output mode 1 . This means that each element of the transmission matrix $\mathrm{T}$ is multiplied for the propagation loss term $L_{i j}$ of path from mode $i$ to mode $j$, where $L_{i j}=L_{j i}$. This path unbalance affect the estimation of the fiber coupling efficiencies that consequently affect the retrieval of the core response of the processor, making the singular value decomposition not possible to apply.

\subsection{Conclusions}

We have presented a thorough characterization of an integrated photonic processor with $8 \times 8$ input and output modes. Characterization and calibration of all of the involved 120 tunable phase shifters and 128 directional couplers is carried out via 


\section{A reconfigurable linear optical circuit}

transmission measurements, in combination with sequential tuning and setting phase shifters in a particular order. Based on these measurements and calibration, for testing the degree of universality of processor, we have characterized the fidelities that can be obtained in implementing Haar random unitary transformations.

Regarding the characterization of the thermo-optic phase shifters, we find that they behave as expected given the designed heater length, and when obeying the current safety settings of the electronic control system. To increase the tuning range to a full period, regarding the former, a redesign and re-fabrication of the processor is needed while, regarding the latter, the maximum heater current needs to be explored and extended, possibly with resistive materials that can withstand higher temperatures.

We find that the thermo-optic crosstalk of the heater-based tunable elements is negligible, causing only $1 \%$ variation in transmitted power. The fiber connectors provide a good degree of reproducibility of the optical response of the processor and the fiber-to-waveguide coupling provides on average an efficiency of $34 \%$.

Investigating the spectral response of the processor we find that it is transparent over a wide wavelength range, i.e., from 1200 to $1750 \mathrm{~nm}$. The processor shows wide reconfigurability within a range of about $150 \mathrm{~nm}$ and shows very small spectral variation in a $10-\mathrm{nm}$ wide range around $1550 \mathrm{~nm}$ wavelength.

We investigated the possibility of optimizing the processor response to overcome fabrication imperfections such that the processor would behave closer to an ideal one. We find that the optimization of transformations with initial high fidelity is limited due probably to approximation errors and/or limited computational resolution of the optimization algorithm. We performed a large number of Haar random transformations by decomposing them into phase settings for our architecture. We demonstrated an optimization method that is in principle able to overcome a certain extent of fabrication imperfections, which improves with the dimension of the chosen sub-matrix. We were able to perform the optimization of the fidelity of the hundred transformations taking into account the restrictions given by current imperfections of the processor. Finally we show that we can improve more than half of the implemented transformations, i.e., the transformations with an initial lower fidelity, with plenty of room for improvement. 


\section{Appendix A}

We report additional information about the calibration of some of the components of the experimental setup used in the characterization of the photonic processor.

In order to know the input optical power injected in the processor we characterize the DFB tunable diode laser used for the processor characterization measurements (DFB BFY diode laser Toptica DLC PRO). The laser head is attached to a 99:1 fiber splitter where the $1 \%$ branch is used for wavelength monitoring via an optical

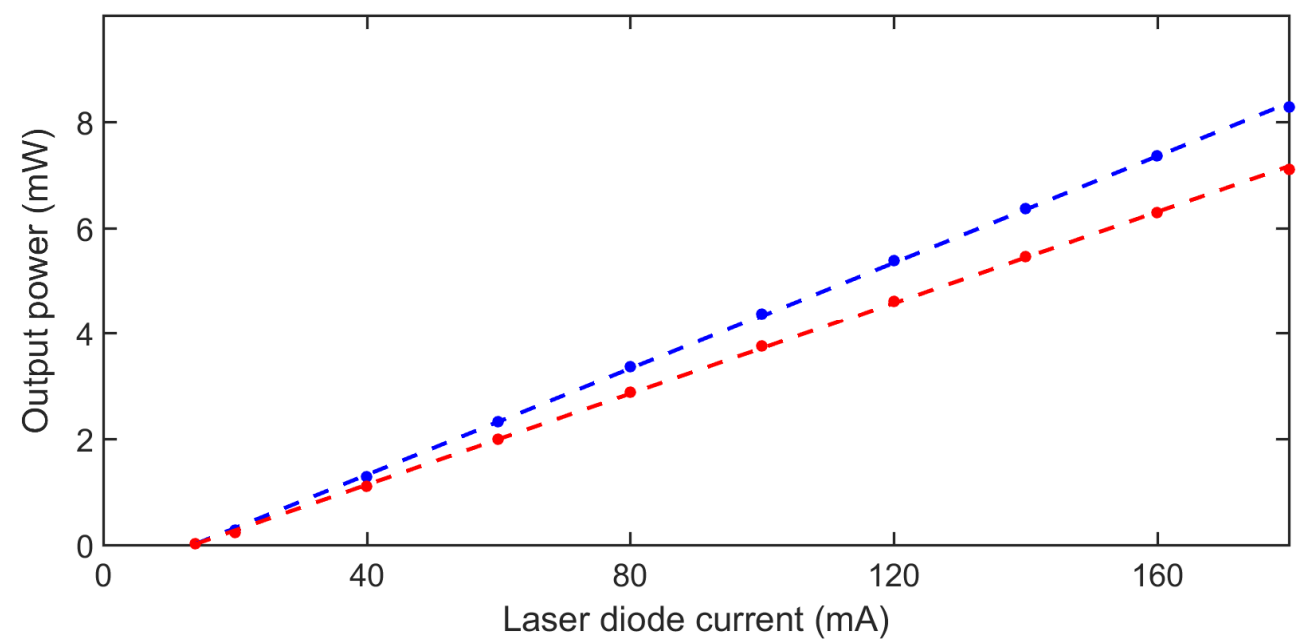

Fig. 4.1A Laser output power versus diode current. Experimental data (dots) and their linear fit (dashed line). The response of the laser changed over a period of few months (different colors) making necessary a renormalization of the data to the closest (in time) laser characterization.

spectrum analyzer and the $99 \%$ branch is used for measurements. The laser output power $\mathrm{P}$ (in $\mathrm{mW}$ ), normalized to the $99 \%$ branch, versus the laser diode current $\mathrm{I}(\mathrm{mA})$ is measured by an integrating sphere photodiode sensor (Thorlabs S144C) giving the following linear response (Fig. 4.1A).

The 8 output modes of the processor are connected, via optical fibers, to 8 photodiodes (PDs), each operated in reverse bias $(20 \mathrm{~V})$ and mounted in the circuit

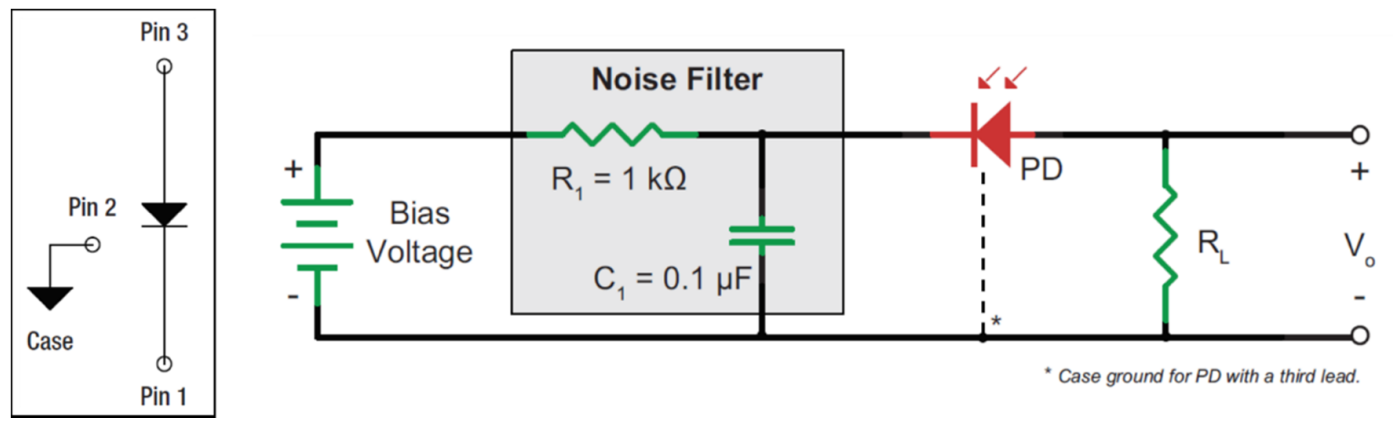

Fig. 4.2A Schematic of the recommended circuit for each 3-leads PD. The load resistor $R_{L}$ is $10 \mathrm{k} \Omega$ assuring that $V_{o}$ is in the range $\pm 10 \mathrm{~V}$ as supported by the NI BNC-2110 card. 
recommended by the manufacturer (Fig. 4.2A). The voltage across the loaded resistance $\mathrm{R}_{\mathrm{L}}$ of each photodiode is red out by connecting each BNC cable to a NI BNC2110 analog-to-digital card plugged via USB to a laptop and recorded, automatized using Labview software.

The dark voltage $\mathrm{V}_{\text {dark }}$ of the PDs is measured by blocking incident light (optical power $\mathrm{P}=0)$ as shown in Fig. 4.3A. In all further power measurements $(\mathrm{P}>0)$ the corresponding dark voltage is subtracted.

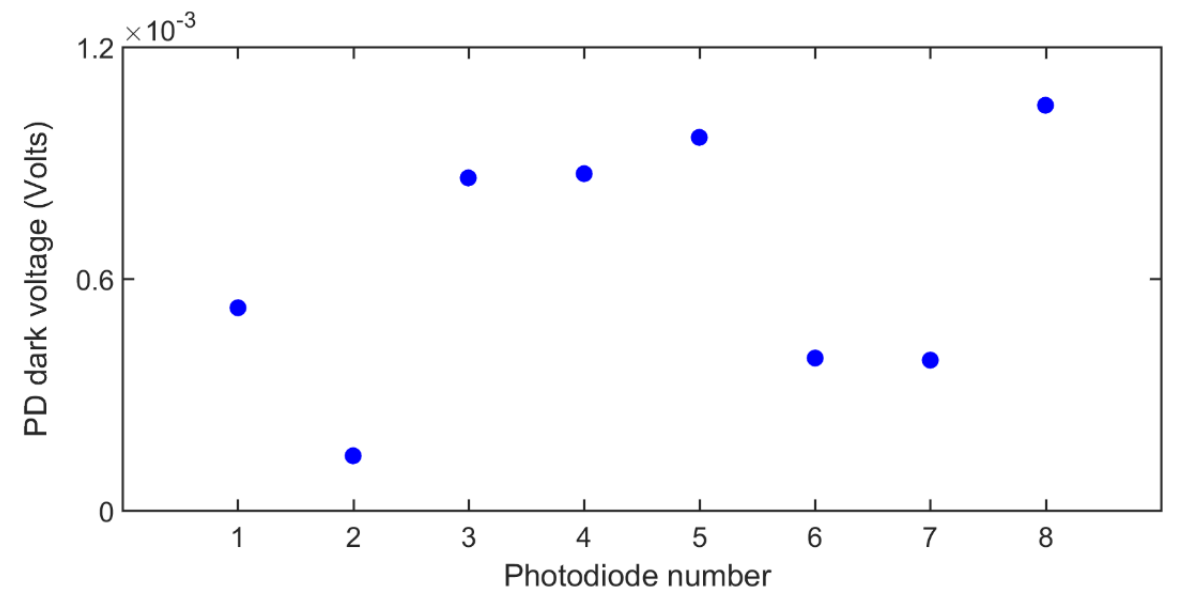

Fig. 4.3A Voltage for incident power $\mathrm{P}=0$ at each $\mathrm{PD}$. Dark count voltage for incident power $\mathrm{P}=0$ at each $\mathrm{PD}$.

When measuring the voltage response of each PD versus the laser diode pump current, we find a linear response over a wide range (Fig. 4.4A), i.e., from the laser's threshold pump current to the saturation of the photodiodes, reached at around $40 \mathrm{~mA}$ (corresponding to an optical power of about $1.5 \mathrm{~mW}$ ). In our experiments, where a greater part of the light is lost mainly at the coupling between fibers and the chip due

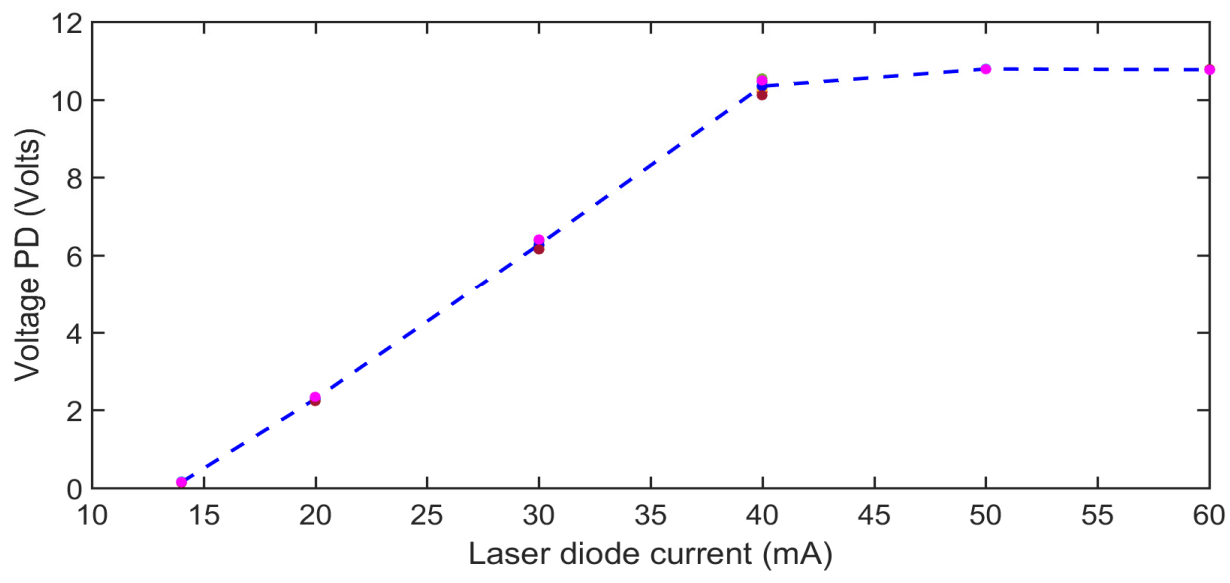

Fig. 4.4A Voltage at the photodiodes (circles of different colors) versus laser diode current. All PDs show a linear response (dashed lined as guide for the eye) reaching saturation for a laser 
to the absence of mode matching, we work clearly in the linear range, far from saturation. 


\section{Appendix B}

Measured transmission matrix $\mathrm{T}$ :

$\left(\begin{array}{llllllll}0,4133 & 0,3794 & 0,3501 & 0,4018 & 0,5410 & 0,6044 & 0,5655 & 0,0689 \\ 0,2414 & 0,3207 & 0,3289 & 0,2711 & 0,4179 & 0,4280 & 0,4881 & 0,0635 \\ 0,4002 & 0,5995 & 0,5246 & 0,5326 & 0,6590 & 0,7102 & 0,6710 & 0,0841 \\ 0,5127 & 0,6067 & 0,7052 & 0,5938 & 0,7270 & 0,7750 & 0,7365 & 0,0843 \\ 0,4853 & 0,6234 & 0,6204 & 0,5933 & 0,7661 & 0,8045 & 0,7632 & 0,0929 \\ 0,5270 & 0,6230 & 0,7122 & 0,6349 & 0,8311 & 0,8912 & 0,8405 & 0,1054 \\ 0,3259 & 0,3638 & 0,3435 & 0,3533 & 0,4500 & 0,5535 & 0,5384 & 0,1038 \\ 0,4150 & 0,5241 & 0,4229 & 0,4747 & 0,6211 & 0,7034 & 0,6425 & 0,0839\end{array}\right)$

Reconstructed matrix M (from singular value decomposition of $\mathrm{T}$ ):

$\left(\begin{array}{llllllll}0,3488 & 0,4266 & 0,4283 & 0,4089 & 0,5283 & 0,5741 & 0,5471 & 0,0704 \\ 0,2695 & 0,3296 & 0,3309 & 0,3159 & 0,4081 & 0,4435 & 0,4227 & 0,0544 \\ 0,4377 & 0,5354 & 0,5375 & 0,5131 & 0,6629 & 0,7204 & 0,6865 & 0,0883 \\ 0,4942 & 0,6044 & 0,6068 & 0,5792 & 0,7484 & 0,8133 & 0,7751 & 0,0997 \\ 0,4973 & 0,6082 & 0,6106 & 0,5829 & 0,7531 & 0,8184 & 0,7799 & 0,1004 \\ 0,5413 & 0,6621 & 0,6647 & 0,6345 & 0,8198 & 0,8909 & 0,8490 & 0,1092 \\ 0,3156 & 0,3859 & 0,3875 & 0,3699 & 0,4779 & 0,5194 & 0,4949 & 0,0637 \\ 0,4080 & 0,4989 & 0,5009 & 0,4782 & 0,6178 & 0,6714 & 0,6399 & 0,0823\end{array}\right)$

The R-squared between the measured transmission matrix $\mathrm{T}$ and the predicted one from the decomposition M of 0.99999897. 


\section{References}

[1] Zhuang, L., et al., "Programmable photonic signal processor chip for radiofrequency applications". Optica 2(10), p. 854-859 (2015).

[2] Pérez, D., et al., "Multipurpose silicon photonics signal processor core”. Nature Communications 8(1), p. 636 (2017).

[3] Marpaung, D., J. Yao, and J. Capmany, "Integrated microwave photonics". Nature Photonics 13(2), p. 80-90 (2019).

[4] Carolan, J., et al., “Universal linear optics”. Science 349(6249), p. 711 (2015).

[5] Harris, N.C., et al., "Quantum transport simulations in a programmable nanophotonic processor". Nature Photonics 11, p. 447 (2017).

[6] Santagati, R., et al., "Silicon photonic processor of two-qubit entangling quantum logic". Journal of Optics 19(11), p. 114006 (2017).

[7] Shen, Y., et al., "Deep learning with coherent nanophotonic circuits". Nature Photonics 11, p. 441 (2017).

[8] Miller, D.A.B., "Self-aligning universal beam coupler". Optics Express 21(5), p. 6360-6370 (2013).

[9] Miller, D.A.B., "Self-configuring universal linear optical component". Photonics Research 1(1), p. 1-15 (2013).

[10] Ribeiro, A., et al., "Demonstration of a 4×4-port universal linear circuit". Optica 3(12), p. 1348-1357 (2016).

[11] Miller, D.A.B., "Perfect optics with imperfect components". Optica 2(8), p. 747750 (2015).

[12] Clements, W.R., et al., “Optimal design for universal multiport interferometers". Optica 3(12), p. 1460-1465 (2016).

[13] Reck, M., et al., "Experimental realization of any discrete unitary operator". Physical Review Letters 73(1), p. 58-61 (1994).

[14] Banerjee, S. and A. Roy, Linear Algebra and Matrix Analysis for Statistics (2014).

[15] Eckstein, A., et al., "Highly Efficient Single-Pass Source of Pulsed Single-Mode Twin Beams of Light". Physical Review Letters 106(1), p. 013603 (2011).

[16] Jin, R.-B., et al., “Theoretical Investigation of a Spectrally Pure-State Generation from Isomorphs of KDP Crystal at Near-Infrared and Telecom Wavelengths". Physical Review Applied 11(3), p. 034067 (2019).

[17] Claudon, J., et al., "A highly efficient single-photon source based on a quantum dot in a photonic nanowire". Nature Photonics 4, p. 174 (2010). 


\section{References}

[18] Pelton, M., et al., "Efficient Source of Single Photons: A Single Quantum Dot in a Micropost Microcavity". Physical Review Letters 89(23), p. 233602 (2002).

[19] Fletcher R. “The Sequential Quadratic Programming Method". In: Di Pillo G., Schoen F. (eds) Nonlinear Optimization. Lecture Notes in Mathematics, vol 1989. Springer, Berlin, Heidelberg (2010). 


\section{Quantum photonic processing on a reconfigurable linear optical circuit}

We demonstrate a reconfigurable $8 \times 8$ integrated linear optical network based on silicon nitride waveguides for quantum information processing. Our processor implements a novel optical architecture enabling any arbitrary linear transformation and constitutes the largest programmable circuit reported so far on this platform. We validate a variety of photonic quantum information processing primitives, in the form of Hong-Ou-Mandel interference, bosonic coalescence/anti-coalescence and highdimensional single-photon quantum gates exploiting the entire functional area of the processor. We achieve fidelities that clearly demonstrate the promising future for large-scale photonic quantum information processing using low-loss silicon nitride.

\subsection{Introduction}

Optical quantum information processing (QIP) aims to solve computational tasks such as quantum simulation [1-4] and quantum machine learning [5, 6] more efficiently than can be done with classical computers, using photons as information carriers and largescale interferometers as processors. To perform increasingly complex tasks on increasingly complex interferometers, maintaining optical path-length stability on a large scale is a central technological challenge.

The key enabling technology for optical QIP is integrated photonics, as it allows linear optical networks to have interferometric stability and, in addition, reconfigurable functionality, via tunable beam splitters and phase shifters. On-chip linear optical networks enable a variety of quantum information and communication protocols such as quantum teleportation [7], on-chip quantum key distribution [8, 9], photonic quantum gates [10] and boson sampling [11-14]. Such structures are also exploited for classical applications, such as machine learning [5, 15-17] and signal processing in microwave photonics [18]. Imperative requirements for on-chip linear optical networks are low propagation loss and high component density.

Amongst the silicon-based platforms, stoichiometric silicon nitride $\left(\mathrm{Si}_{3} \mathrm{~N}_{4}\right)$ offers the unique combination of high index contrast [19] for low bend loss and ultra-low straight-propagation loss [20], enabling thus both dense and low-loss linear optical 
networks (Fig. 5.1) on a compact footprint. $\mathrm{Si}_{3} \mathrm{~N}_{4}$ is a well-known platform for many classical applications [18, 21-24] and shows a mature state-of-the-art technology [25]. Increasingly, silicon nitride is attracting interest in the field of quantum optics for its tremendous potential in implementing on-chip single-photon sources [26, 27], detectors [28, 29], quantum walks [30] and time-bin encoding for quantum communication [31, 32]. Moreover, due to its wide spectral transparency range (from $440 \mathrm{~nm}$ to $2.5 \mu \mathrm{m}$ ), silicon nitride allows interfacing with all common quantum light sources, e.g., spontaneous parametric down-conversion, spontaneous four-wave mixing sources and quantum dots. A clear demonstration of the suitability of this promising platform for realizing large-scale universal programmable linear optical networks is, however, still missing.

In fact, doped silica and silicon-on-insulator technologies host, so far, the majority of on-chip universal linear optical networks for QIP [33]. While the doped silica platform offers low propagation loss only with a larger footprint, i.e., low component density, silicon-on-insulator allows for dense optical circuits at the expense of higher

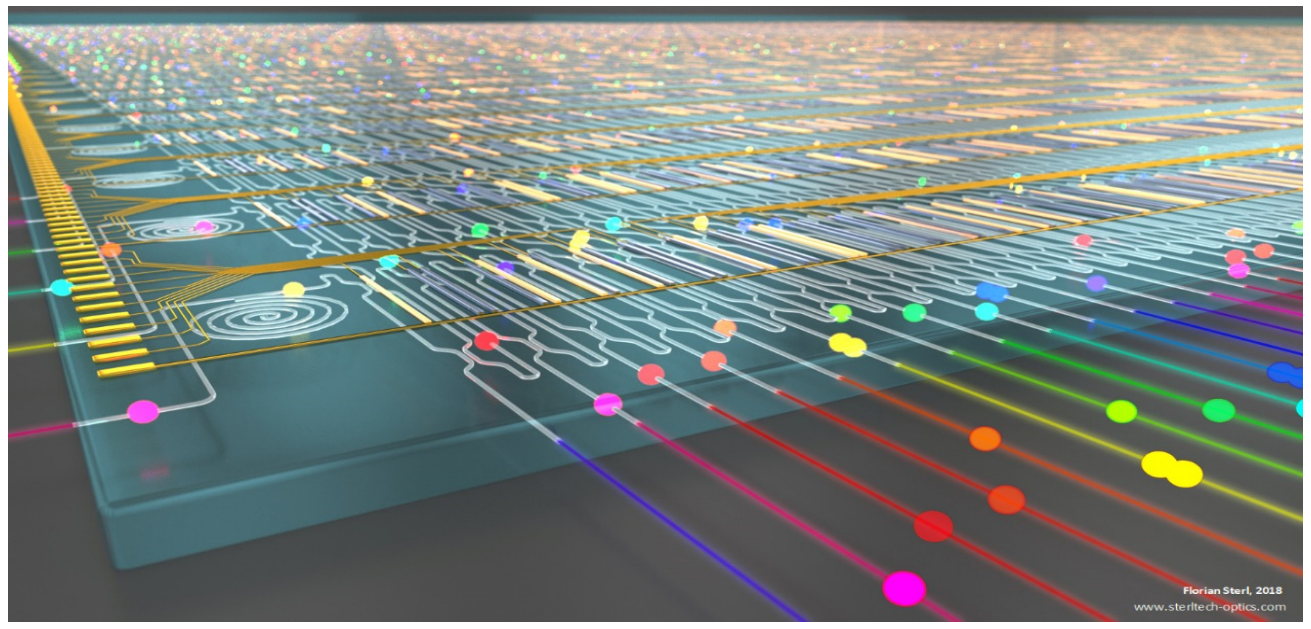

Fig. 5.1 Artist's impression of a silicon nitride waveguide programmable linear optical network. The high index contrast enables a dense waveguide arrangement with the unique combination of ultra-low straightpropagation loss. The wide spectral transparency range makes silicon nitride suitable for quantum light sources from the visible to the mid-infrared.

loss. Since large scale photonic circuits for QIP require both low propagation losses and high component density, a platform that combines these properties is very beneficial for the development of QIP. This combination is what silicon nitride offers.

Here, we demonstrate, for the first time, an $8 \times 8$-mode linear optical network implemented in a photonic processor based on silicon nitride waveguides. The photonic processor is fully reconfigurable over its entire functional area and contains the highest density of components per targeted loss to date [1, 10, 34] (see Section 5.4). The $8 \times 8$-mode photonic processor, the largest realized so far in silicon nitride, includes 
128 reconfigurable elements: 64 tunable beam splitters, constructed as Mach-Zehnder interferometers with internal thermo-optic phase shifters, and 64 additional phase shifters, arranged in a novel linear optical architecture. The processor contains the optical realization of a Blass matrix [35, 36], a well-known architecture for beamforming networks in microwave engineering, where it is used for directional transmission of radio frequency signals to and from antenna arrays. Translating this architecture from microwave engineering to optical QIP, the Blass matrix supports the realization of any arbitrary linear transformation, both unitary $[37,38]$ and non-unitary [39-41]. We show that our processor preserves the coherence of quantum states by programming the processor to implement quantum interference. As a proof-ofprinciple demonstration of the architecture's capability to implement non-unitary transformations, we show anti-coalescence of bosons [42-44] on a $2 \times 2$ Blass matrix. Finally, we realize high-dimensional single-photon quantum gates exploiting the whole spatial mode structure of the processor.

\subsection{Experimental setup}

Figure 5.2 shows a schematic of the experimental setup. The photonic processor (Fig. 5.2(a)) consists of 64 unit cells, each composed of a phase shifter (in red) and a tunable beam splitter (in blue), in an arrangement that enables any arbitrary $8 \times 8$ transformation. Each tunable beam splitter is constructed as a Mach-Zehnder

(a)

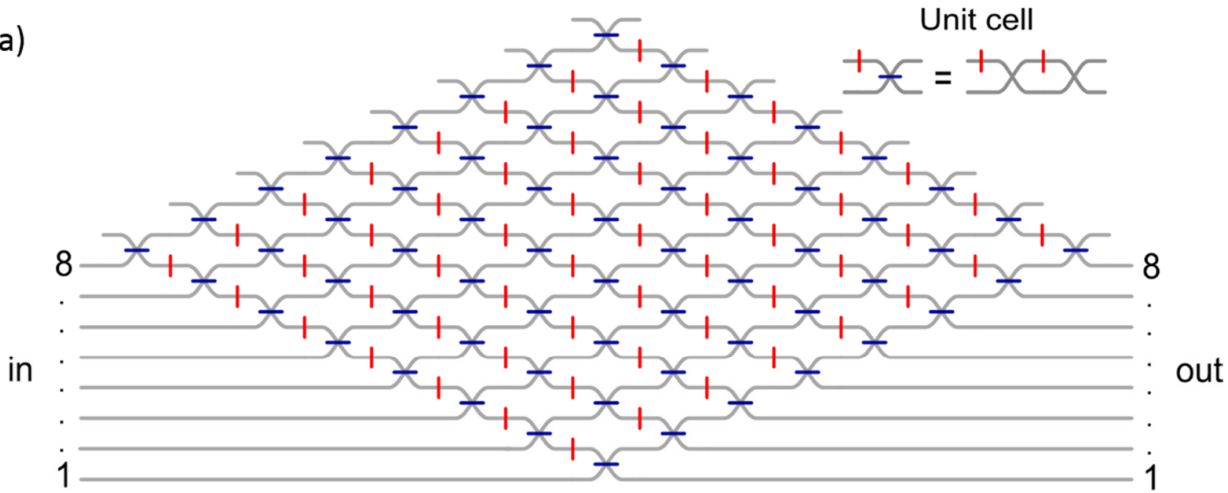

(b)

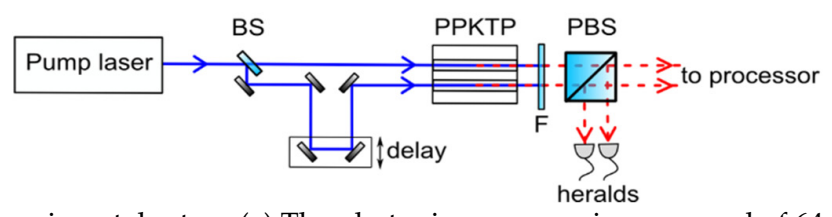

Fig. 5.2 Schematic of the experimental setup. (a) The photonic processor is composed of 64 unit cells, each comprising a phase shifter (red vertical line) and a tunable beam splitter (blue horizontal line) implemented as a Mach-Zehnder interferometer. The rhomboidal shape of the processor's schematic has been chosen for better overview. In the real processor the elements are arranged on a square mesh. (b) Photon pairs are generated via type-II parametric down-conversion in PPKTP waveguides pumped with a mode-locked laser at $775 \mathrm{~nm}$ and injected into the photonic processor. 
interferometer, based on two directional couplers and an internal thermo-optic phase shifter. The 128 thermally tunable elements are remotely controllable and are designed

to each allow a $\frac{3}{2} \pi$ phase shift. The tuning range can be extended with longer tunable elements or stronger current supplies. The photonic processor is based on stoichiometric silicon nitride waveguides, grown with low-pressure chemical vapor deposition, with a double-stripe cross-section [25]. The waveguides exhibit a propagation loss of $0.2 \mathrm{~dB} / \mathrm{cm}$ for a total on-chip transmission greater than or equal to $60 \%$, a value that corresponds to the longest optical path. The coupling losses to a single-mode optical fiber are about $2.9 \mathrm{~dB} /$ facet which can be greatly reduced (to 0.5 $\mathrm{dB} /$ facet [25]) by waveguide tapering.

Single photons for the experiments are provided with two parametric downconversion sources (Fig. 5.2(b)). Frequency-doubled light from a mode-locked fiber laser, with a center wavelength of $775 \mathrm{~nm}$ and a spectral width of $2 \mathrm{~nm}$, is divided into two paths, one containing an adjustable delay line, and focused into two 10-mm-long periodically-poled $\mathrm{KTiOPO}_{4}$ (PPKTP) waveguides [45]. Each PPKTP waveguide generates, via type-II down-conversion, orthogonally-polarized spectrally-separable photon pairs at telecom wavelengths (signal and idler at $1547 \mathrm{~nm}$ and $1553 \mathrm{~nm}$, respectively). After removal of the pump wavelength (F filter for pump rejection) the signal and idler photons are separated using a polarizing beam splitter (PBS) and collected by single-mode fibers. The signal photons are used to herald the idler photons with a heralding efficiency of $30 \%$. The two idler photons are coupled into the photonic processor using polarization-maintaining fibers. For photodetection, we use a set of fiber-coupled superconducting single-photon detectors (efficiency 85\% [46]).

\subsection{Experimental results}

\section{On-chip quantum interference}

To demonstrate the suitability of the photonic processor for QIP, we first observe Hong-Ou-Mandel (HOM) interference [47] between two photons (bullets of the same color in Fig. 5.3(a)) at various positions (beam splitters) within the processor (colored disks in Fig. 5.3(a)). The processor is configured to route the two incident photons across the chip to a targeted beam splitter, which is programmed to a reflectivity of $50 \%$, after which the photons are directed to two outputs. The coincidence count rates at the outputs versus the relative delay of the two single photons are recorded. In Fig. 5.3(b) we show, as an example, the HOM interference at one of the targeted tunable beam splitters (red curve), at the center of the processor in comparison with a reference 
measurement, i.e., an off-chip HOM experiment using a fiber beam splitter (blue curve). It can be seen that the two measurements are well in accord. At a mean photon number of 0.01 we measure a reference HOM dip visibility $V_{\text {ref }}$ of $81 \%$ between the idler photons of the two sources, with the visibility defined as $V=\left|\frac{C_{d}-C_{i}}{C_{d}}\right|$, where $C_{d}$ and $C_{i}$ are respectively the coincidence counts for temporally distinguishable and indistinguishable photons. We repeat the experiment at various positions, i.e., beam splitters, within the photonic processor obtaining similar results, i.e., an average visibility of $76 \%$. The consistency of the measured on-chip HOM dips, over the whole processor depth, with the reference shows that our photonic processor preserves the spectro-temporal similarity of the photons and confirms the suitability of the photonic processor for quantum information processing.

\section{Arbitrary linear transformations}

Due to its architecture, the photonic processor can be configured to perform arbitrary linear transformations on its 8 modes, both unitary and non-unitary [39-41], the latter implemented via ancillary modes. In QIP, non-unitary, lossy, transformations are typically considered detrimental. However, the additional freedom obtained by removing the restriction of unitarity allows for new transformations that exhibit exciting behavior such as a tunable quantum interference and an apparent nonlinear absorption [43, 48, 49]. Already the simple case of a balanced symmetric lossy beam splitter contains free parameters determining the relative phase of the transmission coefficients that enable the tuning of the well-known HOM-like dip, the signature of bosonic coalescence, into a HOM-peak for bosonic anti-coalescence.

To illustrate how the Blass matrix architecture allows the implementation of nonunitary transformations, we realize a balanced symmetric lossy beam splitter, involving four beam splitters and two phase shifters (see Fig. 5.3(c)), described by the

matrix $T=\frac{1}{2}\left(\begin{array}{cc}1 & 1 \\ 1 & e^{i \alpha}\end{array}\right)$, with the phase $\alpha$ a free parameter. The behavior observed in a quantum interference experiment between two single photons will oscillate between coalescence and anti-coalescence of the photons depending on this phase $\alpha$ [49], with the well-known HOM-like coalescence for $\alpha=\pi$. The photonic processor is programmed to perform such a non-unitary $2 \times 2$ transformation on a $2 \times 2$ Blass matrix. Fig. 5.3(d) shows the quantum interference between two single photons for two different non-unitary $2 \times 2$ transformations implemented on the chip, resulting in bosonic coalescence for phase $\alpha=\pi$ (red) and anti-coalescence for $\alpha=-0.52 \mathrm{rad}$ (blue). The visibility of these HOM-like dip and peak are $81 \%$ and $70 \%$, respectively, as expected for these specific transformations. 


\section{High-dimensional quantum logic gates}

High-dimensional quantum states, i.e., qudits, are of importance for large-alphabet quantum communication protocols [50] and cryptography [51]. In optics, qudits can be implemented using a modal degree of freedom, spatial or temporal, of the single photon to encode information. When encoding in the spatial degrees of freedom, large unitary linear optical networks can be exploited to implement high-dimensional quantum logic gates for the control and manipulation of such qudits [52, 53]. As shown in [52], providing all the integer powers of a d-dimensional $X$-gate, i.e., $X, X^{2} \ldots X^{d}$, and of a d-dimensional Z-gate, enables any unitary operation in a d-dimensional state space, with $d=8$ in our case, where the action of a d-dimensional X-gate can be described as $X|j\rangle=|j+1 \bmod d\rangle$ (see Appendix).

Here we demonstrate the realization of an 8-dimensional X-gate (see Fig. 5.3E) and all its integer powers, i.e., $\mathrm{X}, \mathrm{X}^{2} \ldots \mathrm{X}^{8}$, in an 8-dimensional-rail encoding thus exploiting the whole mode structure of the processor. Figure 5.3(f) shows the measured truth table for the X-gate, obtained by injecting single photons into each of the 8 inputs. The results for the integer powers of the X-gate are summarized in Fig. 5.3(g). The fidelities of these gates are about $\mathcal{F}_{8 \times 8}=94.6 \%$, where the fidelity of each gate is calculated as the average state fidelity $\mathcal{F}=\sum_{\mathrm{i}} \frac{\sqrt{\mathrm{p}_{\mathrm{i}}^{\text {exp }} \cdot \mathrm{p}_{\mathrm{i}}^{\text {th }}}}{8}$, with $\mathrm{p}_{\mathrm{i}}^{\text {th }}$ and $\mathrm{p}_{\mathrm{i}}^{\text {exp }}$ being the theoretical and experimental probabilities for each computational input $i$, respectively. Finally, we measure the transformation of a single photon in the coherent superposition state $\frac{1}{\sqrt{2}}\left(|1\rangle_{5} \pm|1\rangle_{6}\right)$ through a 6-dimensional X-gate, with a measured gate fidelity of $\mathcal{F}_{6 \times 6}=96.2 \%$. Figure 5.3(h) shows the action of the 6-dimensional X-gate on the coherent superposition input state showing that the gate preserves the relative phase of the state. 


\subsection{Experimental results}

\section{Quantum interference}

(a)

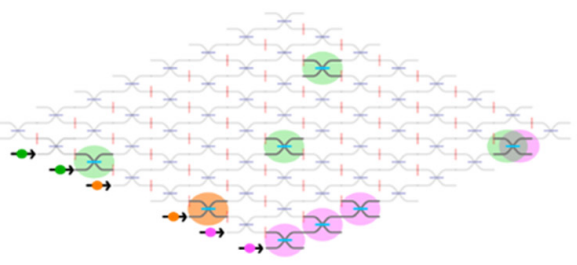

Bosonic coalescence/anti-coalescence

(c)

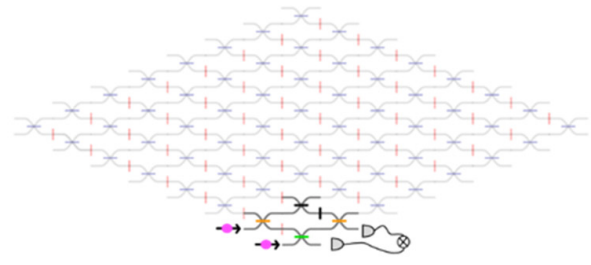

High-dimensional single-photon quantum gates

(e)

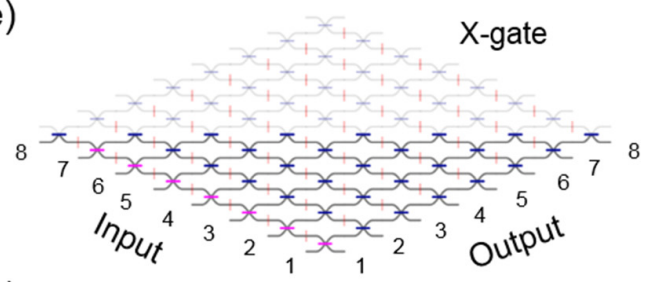

(g)
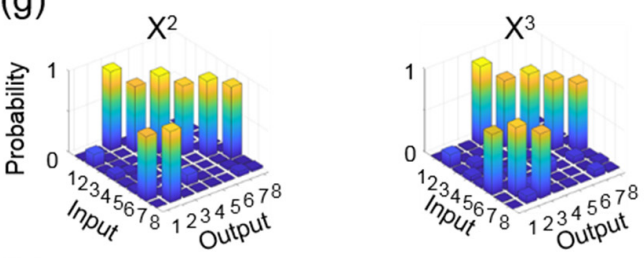

(h)
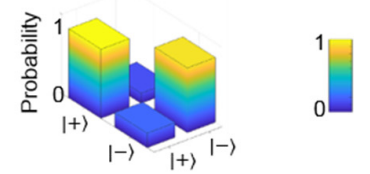

(b)

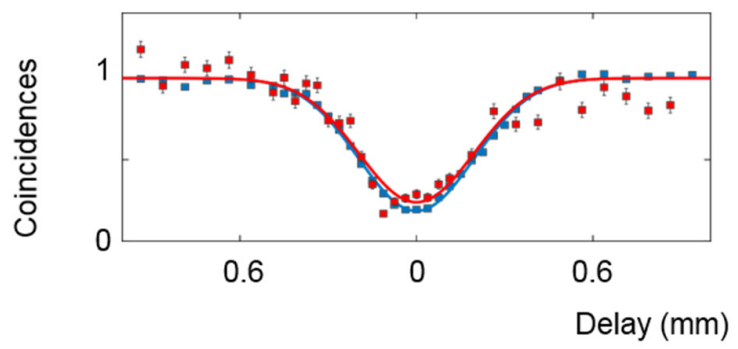

(d)

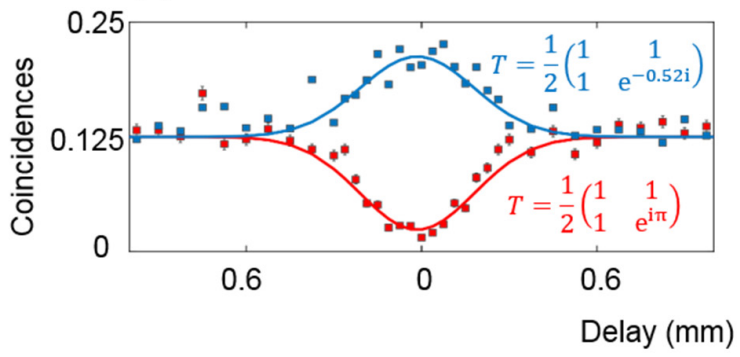

(f)
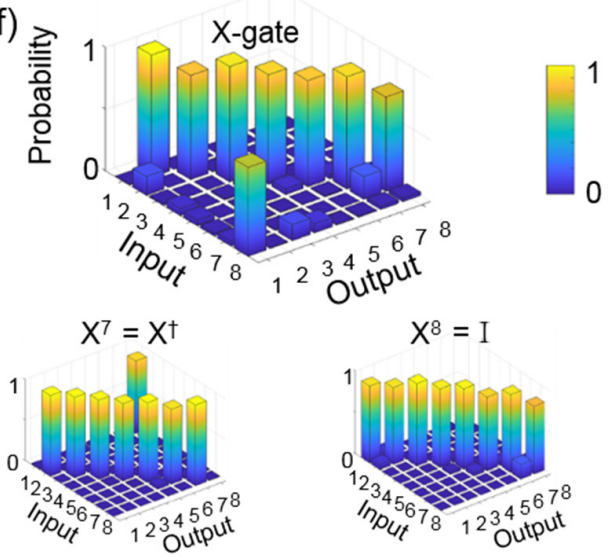

$X^{8}=I$

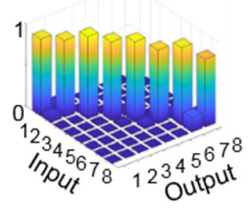

\begin{tabular}{|l|}
\hline Reflectivity \\
$-=0$ (cross) \\
$-=1$ (bar) \\
$-=0.5$ \\
$-=0.25$ \\
$-=0.33$ \\
\hline
\end{tabular}

Fig. 5.3 (a) Two-photon interference at various locations of the processor (colored circles), also indicating the used pairs of input waveguides (bullets of the same color). (b) Coincidence probability versus delay. The two-photon interference measured at the tunable beam splitters on the processor (red data points) is well in accord with the off-chip reference measurement (blue data points). All the investigated beam splitters show a similar visibility. The solid curves indicate Gaussian fits to the data. The error bars are given by the square root of the number of coincidences. (c) Implementation of a lossy beam splitter on a $2 \times 2$ Blass matrix. The black elements are set accordingly to the desired transmission matrix T. (d) Coincidence versus delay for two different lossy transformations T. Measured two-photon bosonic coalescence (red curve)/anti-coalescence (blue curve) with a visibility of $81 \%$ and $70 \%$ respectively. (e) Realization of an 8-dimensional X-gate and (f) its measured truth table. (g) Truth tables of integer powers of the X-gate reported above. The average fidelity is $\mathcal{F}_{8 \times 8}=94.6 \%$. (h) Evolution of a coherent superposition input state $| \pm\rangle=(1 / \sqrt{2})\left(|1\rangle_{5} \pm|1\rangle_{6}\right)$ through a 6-dimensional X-gate, giving a fidelity of $91.9 \%$. 


\subsection{Functional complexity}

In the variety of on-chip universal linear optical networks presented so far, two conflicting developments can be recognized. To achieve higher degrees of functionality, i.e., higher functional complexity, an increasing number of functional elements, e.g., tunable or switchable, is required. Since the size of integrated optical chips is constrained to a chip or wafer by fabrication technology, the density of components on photonic chips can ultimately only be increased via a reducing the size of components and making sharper bends in the waveguides. On the other hand, it remains a central requirement to maintain the lowest propagation loss also with a growing number of components and therefore growing optical path lengths, particularly for quantum processing schemes. Since tunability, component size and optical loss are intrinsically coupled properties of the optical materials and fabrication technology used, the future of photonic processors depends critically on which material platform will enable the greatest functional complexity for a given level of acceptable loss.

An impressive variety of quantum photonic processors has been demonstrated with different material platforms. A most prominent representative for semiconductor materials is silicon-on-insulator (SOI) as employed for demonstration of, e.g., bosonic transport simulation [1]. The advantage of the SOI platform is that it supports extremely dense photonic circuits, thanks to its high index contrast between the waveguide core and cladding $(\Delta \mathrm{n} \approx 40 \%$ [19]), because this allows for small feature size via tight bending radii without much radiation loss. Also, due to the relatively small bandgap, highly responsive tuning elements, with typically $\pi$ phase shift within less than $100 \mu \mathrm{m}$ propagation length, can be realized using carrier injection [54]. On the other hand, surface scattering in combination with crystallinity and the high index contrast enhances the optical propagation loss in SOI to levels of several $\mathrm{dB} / \mathrm{cm}$, both in straight and curved waveguide sections. Additional loss occurs as the drawback of high responsivity in tuning, due to free-carrier absorption [55].

In contrast to semiconductors, amorphous dielectric materials with large bandgap are known for lowest propagation loss, the most well-known representative being doped silica. This platform has been used, for instance, for demonstrating universal linear optical circuits [10]. The typical propagation loss is at least an order of magnitude lower than in SOI, at the level of $0.1 \mathrm{~dB} / \mathrm{cm}$ [19], and thermal tuning can be applied without inducing noticeable additional loss. However, the disadvantage is the low index contrast which is inherent to waveguide cores based on doping $(\Delta \mathrm{n} \approx 0.5 \%$ 
[19]). The low contrast leads to weak guiding and severe radiation loss occurs at smaller curvatures, which makes dense and thus complex waveguide circuits unfeasible.

The work we present here makes use of an advanced dielectric waveguide platform involving waveguide cores made from stoichiometric silicon nitride, embedded in a cladding made from stoichiometric silicon oxide. Based on slow deposition at high temperatures (low-pressure chemical vapor deposition), these waveguides offer a unique combination of high index contrast $(\Delta \mathrm{n} \sim 18 \%$ [19]) and ultralow propagation loss $(>0.0004 \mathrm{~dB} / \mathrm{cm}$ [20]). The platform thus offers ideal preconditions for the realization of dense and low-loss photonics circuits, where tuning-induced loss can be neglected.

In the following, we inspect the different degrees of complexity achievable with the named photonic platforms. For a quantitative comparison, we estimate the maximum achievable complexity with SOI, doped silica and $\mathrm{Si}_{3} \mathrm{~N}_{4}$ in terms of a new figure of merit, $\mathrm{C}_{\mathrm{f}}$. We define this figure as the maximum number of functional unit cells, $\mathrm{n}^{2}$, that can be arranged in a 2D square mesh, before the intensity of the light has dropped to a fraction $\mathrm{f}$. For convenience and definiteness, we proceed with a specific value for this fraction, $\mathrm{f}=\mathrm{e}^{-1}$; however, any other value can be selected as desired.

Next, for comparing universal photonic processors, we define a prototype waveguide circuit as unit cell, with many unit cells forming the processor. As has been shown, all unitary transformations can be implemented using a network of unit cells that can perform two essential functions, tunable beam splitting followed by tunable phase shifting [37].

The most basic functional design of such a unit cell in the form of a 2D waveguide circuit is displayed in Fig. 4. We consider the beam splitter realized with two directional couplers in the form of a Mach-Zehnder interferometer (MZI) where the splitting ratio can be tuned via a phase shifter in one of the interferometer arms. An additional phase shifter in one of the output waveguides allows tuning of the relative phase between the two outputs of the MZI. The optical loss caused by a single unit cell depends on some basic geometrical parameters, specifically, the propagation length through straight waveguide sections where phase tuning is provided, $\mathrm{L}_{\mathrm{t}}$, and the propagation length through sections that are bent with a certain radius $R, L_{b}=4 \pi R$.

The optical loss associated with the various waveguide sections depend on the chosen waveguide platform as described above and on the chosen core cross section of the waveguides. The latter dependence means that the intrinsically lowest-loss of a specified circuit fabricated with a given material platform can only be approached by 
a variation of the core cross section along propagation. Specifically, for each waveguide section depending on its curvature, a different cross section has to be chosen that minimizes the propagation loss. In section 5.5 we describe how we have obtained for each of the three platforms an empiric relation between the waveguide bending radius and the minimum achievable power loss constant, $\alpha_{b}(R)$, specified in unit of $\mathrm{dB} / \mathrm{m}$. Having defined a waveguide circuitry for the unit cell of a photonic processor in terms of waveguide lengths and curvatures then allows to estimate the number of unit cells to be passed before the specified loss fraction, $\mathrm{f}$, is reached.

When analyzing the generic unit cell shown in Fig. 5.4, its path length, over which light propagates, is $\mathrm{L}_{\mathrm{uc}}=\mathrm{L}_{\mathrm{b}}+2 \mathrm{~L}_{\mathrm{t}}$. Here we have assumed for simplicity that the circuit makes use of $90^{\circ}$-bends and that the length of the directional couplers can be neglected compared to the length of tunable and other straight sections.

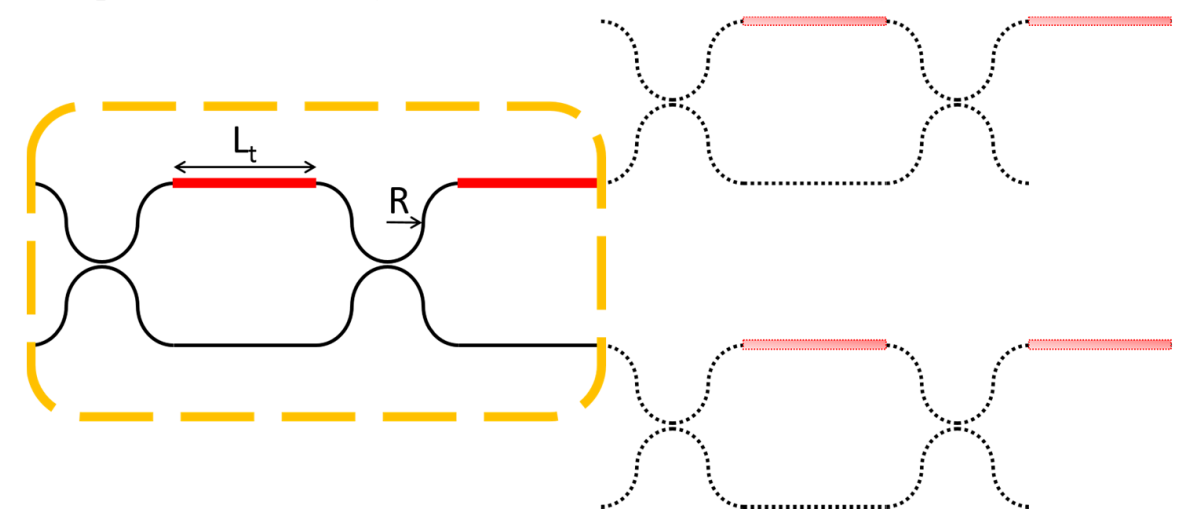

Fig. 5.4 Schematic of a universal linear optical network. The unit cell (dashed frame) comprises a tunable beam splitter in the form of a tunable Mach-Zehnder interferometer with two directional couplers and a phase shifter in one arm of length $L_{t}$ (red), followed by an external phase shifter $\left(L_{t}\right.$, red). The path length through the unit cell is determined by the length of the straight-waveguide tunable elements and the length of curved waveguides (radius of curvature R).

The number of unit cells, $\mathrm{n}$, that can be coupled in series until the power transmission is reduced to $\mathrm{e}^{-1}$ is found by solving the following equation $\mathrm{T}_{\mathrm{n}}=$ $\exp [-(n \cdot \alpha \cdot L \cdot \ln (10)) / 10]=\mathrm{e}^{-1}$ for $n$. In this expression $\alpha$ specifies the power loss for the sections with straight-propagation, with phase-tuning and for bent waveguide sections, respectively $\alpha_{s}, \alpha_{t}$ and $\alpha_{b}$, and where $L_{s}, L_{t}$, and $L_{b}$ are the according lengths of the sections. The total propagation losses are thus given by $\alpha \cdot \mathrm{L}=\alpha_{\mathrm{s}} \cdot \mathrm{L}_{\mathrm{uc}}+\alpha_{\mathrm{b}}$. $\mathrm{L}_{\mathrm{b}}+\alpha_{\mathrm{t}} \cdot\left(2 \mathrm{~L}_{\mathrm{t}}\right)$. The number of unit cells that can be arranged in a $2 \mathrm{D}$ square mesh obeying the same loss condition, i.e., the figure of merit for maximum functional complexity, becomes $\mathrm{C}_{\mathrm{f}}=\mathrm{n}^{2}=\left(10 /\left(\alpha \cdot \mathrm{L}_{\mathrm{uc}} \cdot \ln (10)\right)\right)^{2}$. 
In Fig. 5.5 we plot the functional complexity $C_{\mathrm{f}}$ for a $2 \mathrm{D}$ mesh versus the bending radius, calculated for different material platforms (different colors). The values used

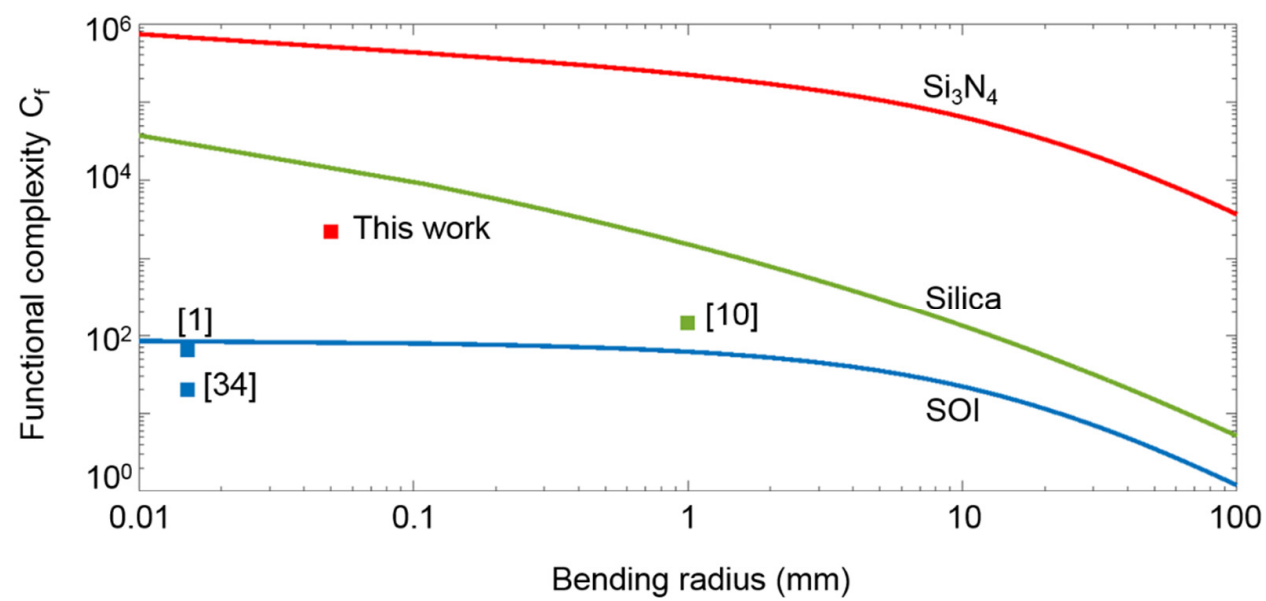

Fig. 5.5 Functional complexity calculated for three different platforms: silicon nitride (red curve), SOI (blue) and doped silica (green). The highest functional complexity achieved in previous work is indicated as data points, i.e., [1][34] realized in SOI, [10] in doped silica and this work realized with silicon nitride.

as loss coefficients, $\alpha_{\mathrm{s}}, \alpha_{\mathrm{t}}$ and $\alpha_{\mathrm{b}}$ and the length of the tunable element are summarized in Table 2 (the coefficients summarize previous experimental data as described in section 5$)$.

It can be seen that silicon nitride provides a functional complexity that is almost four orders of magnitude higher than that of SOI and 1.5 to 3 orders higher than that of silica, depending on the bending radii considered. The much higher propagation loss

\begin{tabular}{|l|c|c|c|c|}
\hline Platform & $\begin{array}{c}\text { Straight- } \\
\text { propagation loss } \\
\alpha_{\mathrm{s}}[\mathrm{dB} / \mathrm{m}]\end{array}$ & $\begin{array}{c}\text { Bending loss } \\
\alpha_{\mathrm{b}}[\mathrm{dB} / \mathrm{m}]^{*}\end{array}$ & $\begin{array}{c}\text { Phase-tuning loss } \\
\alpha_{\mathrm{t}}[\mathrm{dB} / \mathrm{m}]\end{array}$ & $\begin{array}{c}\text { Tunable elements } \\
\text { length } \\
\mathrm{L}_{\mathrm{t}}[\mathrm{mm}]\end{array}$ \\
\hline $\mathrm{SOI}$ & $2.7[56]$ & $4.07 \cdot \mathrm{R}^{-0.62}$ & $3700[54]$ & $0.0616[54]$ \\
\hline $\mathrm{Si}_{3} \mathrm{~N}_{4}$ & $0.045[20]$ & $0.316 \cdot \mathrm{R}^{-0.95}$ & 0 & $12[57]$ \\
\hline Doped Silica & $0.01[58]$ & $7.24 \cdot \mathrm{R}^{-0.74}$ & 0 & $5[59]$ \\
\hline
\end{tabular}

Table 2 Parameters used for the functional complexity calculation.

*The dependence of bending loss vs. waveguide curvature radius is obtained as described in Sect. 5.5.

of SOI is mainly introduced by the tuning via carrier injection [54], even if $\mathrm{L}_{\mathrm{t}}$ can be held much shorter than in $\mathrm{Si}_{3} \mathrm{~N}_{4}$. With regard to doped silica, the higher functional complexity of silicon nitride is due to its substantially lower bending loss. In order to identify and quantify possible room for improvements we compare three recently published realizations, i.e., $[1,10,34]$ and this work, all of them describing on-chip linear optical networks (see data points in Fig. 5.5). The highest complexity of current SOI processors is close to the maximum possible and that of silica can be improved by 
up to two orders of magnitude with smaller radii of curvature. The largest room for improvement is expected for $\mathrm{Si}_{3} \mathrm{~N}_{4}$. Although the complexity of $\mathrm{Si}_{3} \mathrm{~N}_{4}$ is leading already by one order of magnitude, more than three orders of magnitude seem possible.

\subsection{Derivation of loss coefficients}

We recall that radiation loss occurs at all waveguide curvatures (bending loss). When keeping the waveguide cross section constant along the propagation coordinate, the bending loss coefficient, $\alpha_{b}(R)$, increases linearly with $1 / R$ [60][61]. Using short bending radii reduces the propagation length through bent waveguides, so the bending loss, reducing also the area occupied by a circuit. For bent waveguides with increasing radius of curvature, the loss levels off to the minimum value, $\alpha_{s}$, given by the straight-propagation loss, which is usually given by material-intrinsic absorption and Rayleigh scattering.

Both the bending loss and straight-propagation loss are strongly dependent on the index contrast between core and cladding as defined by the selected material platform, by the chosen shape and size of the waveguide core. They depend also largely on the chosen fabrication process. Generally, tightly confining the light to the core with highcontrast waveguides and large, wavelength-sized cross sections, reduces the bending loss but simultaneously increases the straight-propagation loss. Weak guiding on the other hand, as achieved with low index contrast or a small (sub-wavelength) core size in high-contrast materials, can yield very low straight-propagation loss; however, the bending loss becomes significant. In conclusion, the overall loss in a circuit is minimized if the waveguide cross section is continuously adjusted to the local curvature radius. This approach can be seen, e.g., in recent work with silicon waveguides $[62,63]$. 
In order to analyze which waveguide platform offers highest component density at a targeted loss we derive an empirical expression for the minimum value of $\alpha_{b}(R)$ vs the bending radius.

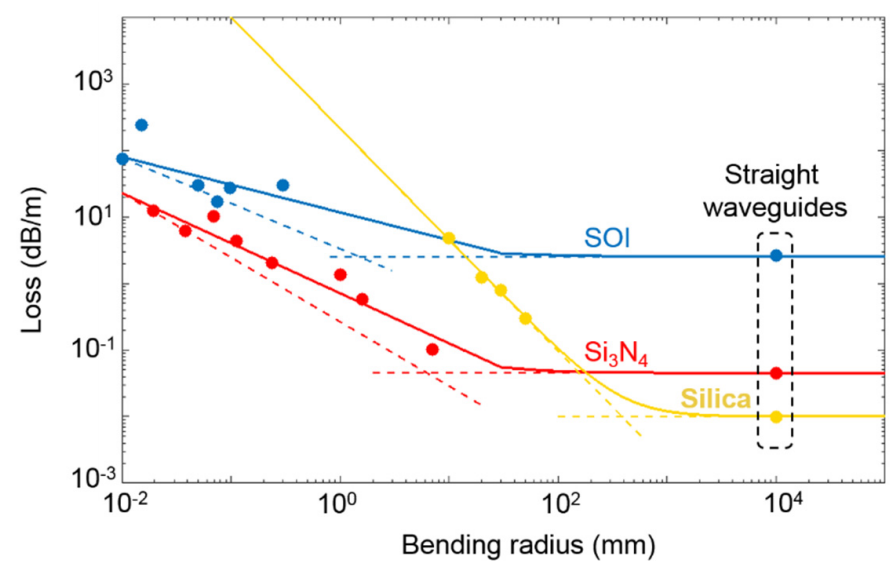

Fig. 5.6 Overview of planar waveguide propagation loss versus bending radius as in [63] with more recent works $[1,62,63,65]$. The loss value for large radius tends to $\alpha_{s}$, the straight-propagation loss.

Figure 5.6 displays experimentally determined loss constants as reported for SOI, $\mathrm{Si}_{3} \mathrm{~N}_{4}$ and doped $\mathrm{SiO}_{2}$ (silica) waveguides vs the fabricated bending radius. Data published until 2014 are taken from [64] and more recent data from [62, 63, 65] and [1]. Although the loss values in the named references show a significant variation, selecting only data points with the lowest loss for each radius and platform yields clear trends. Specifically, the minimum reported loss data vs waveguide bending radius shows that the lowest-loss values follow approximately an inverse power law. We note that this dependence was found approximately also in numerical calculations when adjusting the width and height of rectangular waveguides from silicon, $\mathrm{Si}_{3} \mathrm{~N}_{4}$, and $\mathrm{GeO}_{2}$-doped silica waveguides [64]. Fitting inverse power laws to the lowest-loss experimental data is thus consistent with the assumption that each lowest-loss experimental observation was based on choosing an optimum waveguide cross section for the corresponding bending radius. The coefficients extracted for each platform from the power-law fits are listed in Table 1.

As reported above, with increasing radius of curvature, the value of $\alpha_{b}(R)$ levels off to a constant offset value, which is the minimum loss for straight waveguides $\alpha_{s}$. To indicate these levels, we have drawn in Fig. 6 horizontal dashed and solid lines that pass through the lowest reported experimental loss values (data points in the dashed frame taken from [20] for $\mathrm{Si}_{3} \mathrm{~N}_{4}$, from [56] for SOI and from [58] for doped silica). Adding the according loss constants, $\alpha_{\mathrm{s}}$, to the inverse power law functions then yields a closed expression for the expected minimum loss vs bending radius (solid curves in Fig. 5.6). 
5. Quantum photonic processing on a reconfigurable linear optical circuit

Eventually, for a complete description of losses in tunable and programmable photonic processors, also the loss in the phase-tunable sections of a waveguide circuit has to be quantified, $\alpha_{t}$ (which we term tunability-induced loss). For thermo-optic tuning using large-bandgap dielectric materials, here doped silica and $\mathrm{Si}_{3} \mathrm{~N}_{4}$, no additional losses are expected or have been reported. To implement highly effective tuning in semiconductors within short propagation lengths, carrier injection can be applied, however, this increases the propagation loss through free-carrier absorption [54]. For the waveguide cross sections to be used we assume standard values, i.e., $220 \mathrm{~nm}$ thickness for SOI waveguides [56], and weakly guiding high-aspect ratio waveguides for $\mathrm{Si}_{3} \mathrm{~N}_{4}$ [20] and doped silica [66].

\subsection{Conclusions}

We report the realization of a fully programmable and remotely controllable $8 \times 8$-mode photonic processor, which is the largest universal linear optical network realized in $\mathrm{Si}_{3} \mathrm{~N}_{4}$. We have demonstrated on-chip a variety of QIP primitives such as HOM interference, bosonic anti-coalescence on a $2 \times 2$ Blass matrix and high-dimensional single-photon quantum gates. The obtained results show that our processor retains the indistinguishability of the photons, limited only by the off-chip single-photon source, and enables any arbitrary linear transformation. Our findings demonstrate the promising future of the $\mathrm{Si}_{3} \mathrm{~N}_{4}$ platform for the development of large reconfigurable universal linear optical quantum circuits. 


\section{Appendix}

Figure 5.1A shows the schematic of how to implement any integer power of a ddimensional Pauli X-gate with a d-dimensional linear optical network in Reck's scheme [37] using d-dimensional rail encoding. The linear optical network is described by the schematic in Fig. 5.1A where the element $e_{i, j}$ indicates the tunable beam splitter at the $i^{\text {th }}$ row and $j^{\text {th }}$ column of the linear optical network. The $n^{\text {th }}$ power of a ddimensional $X$-gate, i.e., $X^{n}$, can be found by setting the reflectivity of all the beam

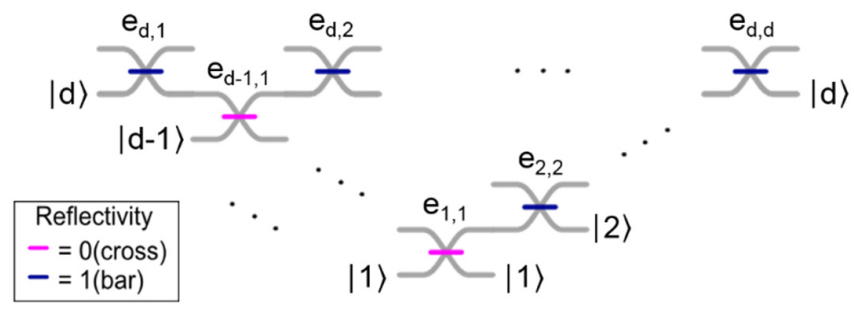

Fig. 5.1A Schematic of how to implement a d-dimensional X-gate on a d-dimensional linear optical network.

splitters $e_{i, j}$ to 1 (bar mode) and changing to 0 (cross mode) the reflectivity of the elements up to the $n^{\text {th }}$ column and up to the $((d-n)+(j-1))^{t h}$ row, for $j=1, \ldots, \mathrm{n}$. 


\section{References}

[1] Harris, N. C. et al. "Quantum transport simulations in a programmable nanophotonic processor". Nature Photonics 11, 447-452 (2017).

[2] Spring, J. B. et al. "Boson Sampling on a Photonic Chip". Science 339, 798-801 (2013).

[3] Crespi, A. et al. "Anderson localization of entangled photons in an integrated quantum walk". Nature Photonics 7, 322-328 (2013).

[4] Carolan, J. et al. "On the experimental verification of quantum complexity in linear optics". Nature Photonics 8, 621-626 (2014).

[5] Ristè, D. et al. "Demonstration of quantum advantage in machine learning". npj Quantum Information 3, 16 (2017).

[6] Biamonte, J. et al. "Quantum machine learning”. Nature 549, 195-202 (2017).

[7] Metcalf, B. J. et al. "Quantum teleportation on a photonic chip". Nature Photonics 8, 770-774 (2014).

[8] Sibson, P. et al. "Chip-based quantum key distribution". Nature Communications 8, 13984 (2017).

[9] Honjo, T., Inoue, K. \& Takahashi, H. “Differential-phase-shift quantum key distribution experiment with a planar light-wave circuit Mach-Zehnder interferometer". Optics Letters 29, 2797-2799 (2004).

[10] Carolan, J. et al. „Universal linear optics”. Science 349, 711-716 (2015).

[11] Broome, M. A. et al. "Photonic Boson Sampling in a Tunable Circuit”. Science 339, 794-798 (2013).

[12] Crespi, A. et al. "Integrated multimode interferometers with arbitrary designs for photonic boson sampling". Nature Photonics 7, 545-549 (2013).

[13] Tillmann, M. et al. "Experimental boson sampling”. Nature Photonics 7, 540544 (2013).

[14] Barkhofen, S. et al. "Driven Boson Sampling”. Physical Review Letters 118, 020502 (2017).

[15] Shen, Y. et al. "Deep learning with coherent nanophotonic circuits". Nature Photonics 11, 441-446 (2017).

[16] Bueno, J. et al. "Reinforcement learning in a large-scale photonic recurrent neural network". Optica 5, 756-760 (2018).

[17] Hughes, T. W., Minkov, M., Shi, Y. \& Fan, S. “Training of photonic neural networks through in situ backpropagation and gradient measurement". Optica 5, 864-871 (2018). 
[18] Zhuang, L., Roeloffzen, C. G. H., Hoekman, M., Boller, K.-J. \& Lowery, A. J. "Programmable photonic signal processor chip for radiofrequency applications". Optica 2, 854-859 (2015).

[19] Harris N., C. et al. "Large-scale quantum photonic circuits in silicon". Nanophotonics 5, 456-468 (2016).

[20] Bauters, J. F. et al. "Planar waveguides with less than $0.1 \mathrm{~dB} / \mathrm{m}$ propagation loss fabricated with wafer bonding". Optics Express 19, 24090-24101 (2011).

[21] Burla, M. et al. "Multiwavelength-Integrated Optical Beamformer Based on Wavelength Division Multiplexing for 2-D Phased Array Antennas". Journal of Lightwave Technology 32, 3509-3520 (2014).

[22] Yurtsever, G. et al. "Photonic integrated Mach-Zehnder interferometer with an on-chip reference arm for optical coherence tomography". Biomed. Optics Express 5, 1050-1061 (2014).

[23] Epping, J. P. et al. “On-chip visible-to-infrared supercontinuum generation with more than $495 \mathrm{THz}$ spectral bandwidth". Optics Express 23, 19596-19604 (2015).

[24] Fan, Y. et al. "290 Hz intrinsic linewidth from an integrated optical chip-based widely tunable InP-Si3 $\mathrm{N}_{4}$ hybrid lased". Conference on Lasers and ElectroOptics (Optical Society of America, 2017), JTh5C.9.

[25] Roeloffzen, C. G. H. et al. "Low-Loss Si3N4 TriPleX Optical Waveguides: Technology and Applications Overview". IEEE Journal of Selected Topics in Quantum Electronics 24, 1-21 (2018).

[26] Ellis, D. J. P. et al. "Independent indistinguishable quantum light sources on a reconfigurable photonic integrated circuit". Applied Physics Letters 112, 211104 (2018).

[27] Zhang, X., Zhang, Y., Xiong, C., Eggleton, B. J. "Correlated photon pair generation in low-loss double-stripe silicon nitride waveguides". Journal of Optics 18, 074016 (2016).

[28] Schuck, C. et al. "Quantum interference in heterogeneous superconductingphotonic circuits on a silicon chip". Nature Communications 7, 10352 (2016).

[29] Schuck, C., Pernice, W. H. P. \& Tang, H. X. “NbTiN superconducting nanowire detectors for visible and telecom wavelengths single photon counting on $\mathrm{Si}_{3} \mathrm{~N}_{4}$ photonic circuits". Applied Physics Letters 102, 051101 (2013).

[30] Peruzzo, A. et al. "Quantum Walks of Correlated Photons". Science 329, 15001503 (2010).

[31] Xiong, C. et al. "Compact and reconfigurable silicon nitride time-bin entanglement circuit". Optica 2, 724-727 (2015). 
[32] Zhang, X. et al. "Integrated silicon nitride time-bin entanglement circuits". Optics Letters 43, 3469-3472 (2018).

[33] Flamini, F., Spagnolo, N. \& Sciarrino, F. "Photonic quantum information processing: a review". arXiv:1803.02790 (2018).

[34] Pérez, D. et al. "Multipurpose silicon photonics signal processor core". Nature Communications 8, 636 (2017).

[35] Blass, J. “Multidirectional antenna - a new approach to stacked beams”. in 1958 IRE International Convention Record (IEEE, 1960), pp. 48-50.

[36] Casini, F., Gatti, R. V., Marcaccioli, L. \& Sorrentino, R. "A novel design method for Blass matrix beam-forming networks". in 2007 European Radar Conference pp. 232-235.

[37] Reck, M., Zeilinger, A., Bernstein, H. J. \& Bertani, P. “Experimental realization of any discrete unitary operator". Physical Review Letters 73, 58-61 (1994).

[38] Clements, W. R., Humphreys, P. C., Metcalf, B. J., Kolthammer, W. S. \& Walmsley, I. A. "Optimal design for universal multiport interferometers". Optica 3, 1460-1465 (2016).

[39] He, B., Bergou, J. A. \& Wang, Z. "Implementation of quantum operations on single-photon qudits". Physical Review A 76, 042326 (2007).

[40] Tischler, N., Rockstuhl, C. \& Słowik, K. “Quantum Optical Realization of Arbitrary Linear Transformations Allowing for Loss and Gain". Physical Review X 8, 021017 (2018).

[41] Miller, D. A. B. "Self-configuring universal linear optical component". Photonics Research 1, 1-15 (2013).

[42] Defienne, H., Barbieri, M., Walmsley, I. A., Smith, B. J. \& Gigan, S. “Two-photon quantum walk in a multimode fiber". Science Advances 2, e1501054 (2016).

[43] Wolterink, T. A. W. et al. "Programmable two-photon quantum interference in $10^{3}$ channels in opaque scattering media". Physical Review A 93, 053817 (2016).

[44] Vest, B. et al. "Anti-coalescence of bosons on a lossy beam splitter". Science 356, 1373-1376 (2017).

[45] Eckstein, A., Christ, A., Mosley, P. J. \& Silberhorn, C. "Highly Efficient SinglePass Source of Pulsed Single-Mode Twin Beams of Light". Physical Review Letters 106, 013603 (2011).

[46] Beyer, A. D. et al. "Tungsten Silicide Superconducting Nanowire Single-Photon Test Structures Fabricated Using Optical Lithography". IEEE Transactions on Applied Superconductivity 25, 1-5 (2015). 
[47] Hong, C. K., Ou, Z. Y. \& Mandel, L. "Measurement of subpicosecond time intervals between two photons by interference". Physical Review Letters 59, 2044-2046 (1987).

[48] Barnett, S. M., Jeffers, J., Gatti, A. \& Loudon, R. “Quantum optics of lossy beam splitters". Physical Review A 57, 2134-2145 (1998).

[49] Uppu, R., Wolterink, T. A. W., Tentrup, T. B. H. \& Pinkse, P. W. H. “Quantum optics of lossy asymmetric beam splitters". Optics Express 24, 16440-16449 (2016).

[50] Fujiwara, M., Takeoka, M., Mizuno, J. \& Sasaki, M. "Exceeding the Classical Capacity Limit in a Quantum Optical Channel". Physical Review Letters 90, 167906 (2003).

[51] Langford, N. K. et al. "Measuring Entangled Qutrits and Their Use for Quantum Bit Commitment". Physical Review Letters 93, 053601 (2004).

[52] Babazadeh, A. et al. "High-Dimensional Single-Photon Quantum Gates: Concepts and Experiments". Physical Review Letters 119, 180510 (2017).

[53] Di, Y.-M. \& Wei, H.-R. “Elementary gates for ternary quantum logic circuit”. arXiv:11055485 (2011).

[54] Harris, N. C. et al. "Efficient, compact and low loss thermo-optic phase shifter in silicon". Optics Express 22, 10487-10493 (2014).

[55] Schroder, D. K., Thomas, R. N. \& Swartz, J. C. "Free carrier absorption in silicon". IEEE Transactions on Electron Devices 25, 254-261 (1978).

[56] Biberman, A., Shaw, M. J., Timurdogan, E., Wright, J. B. \& Watts, M. R. "Ultralow-loss silicon ring resonators". Optics Letters 37, 4236-4238 (2012).

[57] Hai, S. M., Leinse, A., Veenstra, T. \& Liboiron-Ladouceur, O. "A Thermally Tunable $1 \times 4$ Channel Wavelength Demultiplexer Designed on a Low-Loss $\mathrm{Si}_{3} \mathrm{~N}_{4}$ Waveguide Platform". Photonics 2 (2015).

[58] Schroeder, J., Mohr, R., Montrose, C. J. \& Macedo, P. B. “Light scattering in a number of optical grade glasses". Journal of Non-Crystalline Solids 13, 313-320 (1974).

[59] Samadi, P. et al. "RF arbitrary waveform generation using tunable planar lightwave circuits". Optics Communications 284, 3737-3741 (2011).

[60] Heiblum, M. \& Harris, J. “Analysis of curved optical waveguides by conformal transformation". IEEE Journal of Quantum Electronics 11, 75-83 (1975).

[61] E. A. J. Marcatili, "Bends in optical dielectric guides”. The Bell System Technical Journal 48, 7, 2103-2132 (1969). 
[62] Morrison, B., Zhang, Y., Pagani, M., Eggleton, B. \& Marpaung, D. "Four-wave mixing and nonlinear losses in thick silicon waveguides". Optics Letters 41, 2418-2421 (2016).

[63] Guan, B. et al. "CMOS Compatible Reconfigurable Silicon Photonic Lattice Filters Using Cascaded Unit Cells for RF-Photonic Processing". IEEE Journal of Selected Topics in Quantum Electronics 20, 359-368 (2014).

[64] Heck, M., Bauters, J., L. Davenport, M., T. Spencer, D. \& Bowers, J. “Ultra-low loss waveguide platform and its integration with silicon photonics". Laser \& Photonics Reviews 8, 667-686 (2014).

[65] Cardenas, J. et al. "Low loss etchless silicon photonic waveguides". Optics Express 17, 4752-4757 (2009).

[66] Adar, R., Serbin, M. R. \& Mizrahi, V. "Less than $1 \mathrm{~dB}$ per meter propagation loss of silica waveguides measured using a ring resonator". Journal of Lightwave Technology 12, 1369-1372 (1994). 


\section{Conclusions}

In this thesis we have exploited the long-term interferometric stability, low propagation loss, tight optical confinement, and maturity of the $\mathrm{Si}_{3} \mathrm{~N}_{4}$ integrated photonics platform. We have constructed programmable integrated waveguide circuits of high performance and high complexity for an application with classical light (high photon number) and for quantum optical applications based on single photons.

The first part of the work investigated spectral analysis of classical light and mitigation of fabrication imperfections that are inherent to integrated photonics, via neural-network-based modeling and optimization algorithms. Specifically, we investigated tunable microring resonator circuits with neural-network data processing to explore options for applications as on-chip wavelength meter. We have demonstrated, for the first time in this approach, long-term (up to a week) reproducibility of such wavelength meter and, additionally, observed an unexpected immunity of the output reading vs changes of the ambient temperature. Although the origin of this temperature immunity is not yet fully understood, we anticipate that additional spectral features, which are usually not accounted for in simple physical modelling but which enter neural network data processing, can be an explanation for the observed immunity. Introducing more spectral features and readout channels would thus be a promising route to proceed.

For the implementation of additional spectral features and readout channels, forthcoming extensions of programmable waveguide circuits for spectrometric applications [1-4] might involve arrays of high-Q ring resonators and Mach-Zehnder interferometer (MZIs) trees for high-resolution and covering wider spectral ranges, respectively. Combining ring resonators and MZIs tree would allow the achievement of both advantages in integrated-optical devices.

As neural network processing does not necessarily require a detailed traceability of the physical origin of spectral features, we conclude that much promise lies in also developing disordered integrated systems on chip (random waveguide circuits) for spectroscopic purposes. Such circuits have already come of interest for the investigation of random quantum walk [5], transverse localization [6] and signal transport [7]. Such devices [8] would then take the complex spatial distribution of the transmitted light over many output channels as a fingerprint, uniquely identifying the wavelength of the probe signal via neural network and deep learning algorithms. 
Optically, disordered systems can be integrated in the form of unusual waveguide structures, e.g., random resonators arrays or randomly coupled waveguide arrays [9].

As a side note, we remark that random waveguide circuits could also yield an interesting application in the field of quantum-secure authentication. Random waveguide circuits could be considered Physically Unclonable Function (PUFs) [10, 11] given the fact that even the smallest variation and imperfection in the fabrication process will drastically and ultimately change their optical response.

In the second part of the thesis we investigated reconfigurable multibeam interferometers for implementing complex functionalities, again exploiting programmability, tight optical confinement, low-loss and technological maturity of the waveguide platform. We have realized a complex reconfigurable network of waveguide interferometers to be used as a single-pass photonic processor. The $8 \times 8$ mode processor, which is the largest realized so far in the silicon nitride platform, was fully characterized using classical light transmission measurements, i.e., all of the internal components were investigated and calibrated with regard to fidelity and range of programming. We have demonstrated, for the first time, the possibility of mitigating fabrication imperfections of a real processor by optimization of the configuration of phases, leading to improved performance of the processor.

Subsequently we have made use of the processor for performing high-dimensional quantum information processing by feeding the photonic processor with single photons and pairs of single photons and detecting these photons at the outputs. Thanks to the long-term interferometric stability and functional complexity, we were able to demonstrate 8 -dimensional single-photon gates. With pairs of single photons and coincidence detection, we were able to show that our processor conserved the spectro-temporal similarity of the photons. This confirms the suitability of our integrated-photonics $\mathrm{Si}_{3} \mathrm{~N}_{4}$ processor for quantum information processing. Given the combination of high component density and record low loss, we argue that $\mathrm{Si}_{3} \mathrm{~N}_{4}$ can be seen as an emerging platform of choice for scalable integrated-photonics-based quantum photonic processing.

An application where a large photonic processor of the described type can be of high interest is boson sampling: the complex interference of many single photons in a large linear optical network will demonstrate a first case of the so-called quantum advantage in information processing. Similarly, random waveguide circuits may be considered in this context, as they can offer a very high number of interfering waveguides in a compact low-loss circuit.

As an overview on future tasks regarding programmable waveguide circuits for quantum information processing, such as the one presented in Chapter 4 and 5, one can envision the following. 
First, the room offered by the waveguide technology should be systematically exploited for further reducing on-chip propagation loss, and loss in coupling to external components, such as to fibers. Relying on single-photons and coincidence measurements, without the option of amplification as with classical light, quantum information processing is critically dependent on prevention of losses. For instance, the demonstration of a quantum advantage with photonic boson sampling will not succeed if the losses are too high $[12,13]$.

A second task lies in the direction of higher speed in reconfigurability, much beyond what is possible with thermo-optic tuning (millisecond scale). Bringing the speed into the nanosecond range, using long delay lines on-chip, or fiber delay lines might break the limit of photonic processors as single-pass devices, via reconfiguring the processor before a photon output distribution is fed back into the processor for a second computational step. The delay lines would have the function of photonic quantum memories, to store and keep the state of quantum bits unchanged during information processing, as another step towards realizing photonic quantum computers. In this task, systematically lowering the waveguide loss is essential to realize delay lines and high-Q cavities as photonic memories.

A third task would be the integration of single-photon sources, i.e., quantum emitters [14, 15], as well as single-photon detectors, with a quantum photonic processor on the same platform. This would remove limitations with regard to coupling losses and improve interferometric stability, thus enabling a major step towards the full integration of a photonic processor for quantum information processing.

In conclusion, the future of integrated programmable low-loss and high-contrast waveguide circuits points in many diverse directions such as spectrometric applications, microwave photonics [16, 17], on-chip quantum-key distribution [18-21], simulation of molecular dynamics [22], neuromorphic computing [23-26] and quantum information processing [27-29]. Despite their diversity, these fields are facing technological challenges on a common ground.

For example, one challenge is to address the need of high-frequency, compact, low-power and low-loss phase shifters. A second example is increasing the precision in fabrication for improved predictability of the optical circuit parameters and the electronic control of phase shifters. A third example is increasing the density of waveguides by maximally exploiting the high index contrast via adiabatic transitions between different waveguide cross-sections on the same chip, specifically, between straight and bent waveguide paths. In all this it will be important to expand the 


\section{Conclusions}

wavelength range across which low-loss, high-density and high-speed programmable waveguide circuits can be employed. Meeting these challenges will be quintessential for advanced applications of integrated photonics, in which this thesis is meant to contribute. 


\section{References}

[1] Souza, M.C.M.M., et al., "Fourier transform spectrometer on silicon with thermo-optic non-linearity and dispersion correction". Nature Communications 9(1), p. 665 (2018).

[2] Vasiliev, A., et al., "Integrated Silicon-on-Insulator Spectrometer With Single Pixel Readout for Mid-Infrared Spectroscopy". IEEE Journal of Selected Topics in Quantum Electronics 24(6), p. 1-7 (2018).

[3] Yebo, N.A., et al., “On-Chip Arrayed Waveguide Grating Interrogated Siliconon-Insulator Microring Resonator-Based Gas Sensor". IEEE Photonics Technology Letters 23(20), p. 1505-1507 (2011).

[4] Zheng, S.N., L.K. Chin, and A.Q. Liu. "On-chip Spectrometer Enhanced by Ring Resonator Cavity: High-resolution and Large-bandwidth". in Conference on Lasers and Electro-Optics, San Jose, California (Optical Society of America 2017), paper JTh2A.119.

[5] Vieira, R., E.P.M. Amorim, and G. Rigolin, "Entangling power of disordered quantum walks". Physical Review A 89(4), p. 042307 (2014).

[6] De Raedt, H., A. Lagendijk, and P. de Vries, "Transverse Localization of Light". Physical Review Letters 62(1), p. 47-50 (1989).

[7] Yu, S., et al., "Metadisorder for designer light in random systems". Science Advances 2(10), p. e1501851 (2016).

[8] Redding, B., et al., "Compact spectrometer based on a disordered photonic chip". Nature Photonics 7, p. 746 (2013).

[9] Martin, L., et al., "Anderson localization in optical waveguide arrays with offdiagonal coupling disorder". Optics Express 19(14), p. 13636-13646 (2011).

[10] ŠKoriĆ, B., "Quantum readout of Physical Unclonable Functions”. International Journal of Quantum Information 10(01), p. 1250001 (2012).

[11] Goorden, S.A., et al., "Quantum-secure authentication of a physical unclonable key". Optica 1(6), p. 421-424 (2014).

[12] Renema, J., V. Shchesnovich, and R. Garcia-Patron, "Classical simulability of noisy boson sampling". arXiv:1809.01953 (2018).

[13] Renema, J.J., et al., "Efficient Classical Algorithm for Boson Sampling with Partially Distinguishable Photons". Physical Review Letters 120(22), p. 220502 (2018). 
6. References

[14] Senellart, P., G. Solomon, and A. White, "High-performance semiconductor quantum-dot single-photon sources". Nature Nanotechnology 12, p. 1026 (2017).

[15] Coles, R.J., et al., "Chirality of nanophotonic waveguide with embedded quantum emitter for unidirectional spin transfer". Nature Communications 7, p. 11183 (2016).

[16] Marpaung, D., J. Yao, and J. Capmany, "Integrated microwave photonics". Nature Photonics 13(2), p. 80-90 (2019).

[17] Pérez, D., et al., "Multipurpose silicon photonics signal processor core”. Nature Communications 8(1), p. 636 (2017).

[18] Bunandar, D., et al., "Metropolitan Quantum Key Distribution with Silicon Photonics". Physical Review X 8(2), p. 021009 (2018).

[19] Sibson, P., et al., "Chip-based quantum key distribution”. Nature Communications 8, p. 13984 (2017).

[20] Wang, F.-X., et al., "Quantum key distribution with dissipative Kerr soliton generated by on-chip microresonators". arXiv:1812.11415 (2018).

[21] Zhang, G., et al. "Integrated Chip for Continuous-variable Quantum Key Distribution using Silicon Photonic Fabrication". in Conference on Lasers and Electro-Optics, San Jose, California (Optical Society of America 2018), paper FTu3G.2.

[22] Sparrow, C., et al., "Simulating the vibrational quantum dynamics of molecules using photonics". Nature 557(7707), p. 660-667 (2018).

[23] Chakraborty, I., et al., "Toward Fast Neural Computing using All-Photonic Phase Change Spiking Neurons". Scientific Reports 8(1), p. 12980 (2018).

[24] Katumba, A., et al., "Neuromorphic Computing Based on Silicon Photonics and Reservoir Computing". IEEE Journal of Selected Topics in Quantum Electronics 24(6), p. 1-10 (2018).

[25] Cheng, Z., et al., “On-chip photonic synapse”. Science Advances 3(9), p. e1700160 (2017).

[26] Peng, H., et al., "Neuromorphic Photonic Integrated Circuits". IEEE Journal of Selected Topics in Quantum Electronics 24(6), p. 1-15 (2018).

[27] Carolan, J., et al., “Universal linear optics”. Science 349(6249), p. 711 (2015).

[28] Metcalf, B.J., et al., "Quantum teleportation on a photonic chip". Nature Photonics 8, p. 770 (2014).

[29] Zeuner, J., et al., "Integrated-optics heralded controlled-NOT gate for polarization-encoded qubits". npj Quantum Information 4(1), p. 13 (2018). 


\section{Summary}

Programmable waveguide circuits are crucial building blocks for integrated spectrometric applications and quantum photonic information processing. Amongst the dielectric material platforms in integrated photonics, silicon nitride stands out with highly attractive properties such as a large bandgap energy and a moderately high index contrast. This allows low propagation losses in a wide spectral range while simultaneously allowing a dense packing of components. The aforementioned properties, together with the inherent phase stability and phase programmability achievable in silicon nitride, enable the creation of complex photonic circuits. In this thesis we describe and demonstrate densely integrated programmable photonic circuits based on silicon nitride waveguides for wavelength metrology and quantum information processing.

A novel approach based on ring resonators and neural-network-based analysis is presented for realizing a high-precision integrated wavelength meter. A neural network is trained with a set of transmission spectra of an integrated photonic circuit consisting of ring resonators, either a single ring or two rings in Vernier configuration, for various tuning settings of the integrated optical circuit. Subsequently, the transmission of an unknown input wavelength can be measured with the integrated optical circuit using the same settings used during the training. The unknown input wavelength is identified by a data-processing method finding the closest match between the calibrated transmission spectra and the measured one. We investigate various data-processing methods for maximizing the wavelength estimation range of the wavelength meter. We demonstrate long-term reproducibility and observe temperature immunity of the wavelength meter readout against changes of the ambient temperature.

We also present a reconfigurable multibeam interferometer, to be used as a photonic processor, containing 128 tunable elements in a Blass matrix configuration. This interferometer is the largest integrated programmable waveguide circuit realized so far in silicon nitride. We report a thorough characterization of the response of the tunable elements, i.e., their tuning range, the thermal crosstalk across the processor, the reproducibility, core response, dispersion and transparency of the processor. Due to fabrication imperfections, not all of the tunable elements can currently be set to the entire parameter range, which slightly limits the reconfigurability of the processor. 
Despite this fact, the processor holds a consistent reconfigurability over a wide spectral range of about $150 \mathrm{~nm}$.

After the calibration of the tunable elements we inspect the possibility of optimizing the processor response, in spite of the finite tuning range of the tunable elements. We measure the fidelities of 100 unitary transformations sampled by a Haar random distribution and investigate whether the measured fidelities can be improved by optimizing the phase settings of the tunable elements over their constrained phase range. We demonstrate an optimization algorithm that is able to overcome, to a certain extent, fabrication imperfections for a majority of the implemented transformations.

We show that the aforementioned 8-modes circuit based on silicon nitride waveguides can be exploited as photonic processor for quantum information processing. The combination of low propagation loss and high index contrast makes silicon nitride very attractive for large-scale quantum information processing. Furthermore, due to its wide spectral range, silicon nitride allows interfacing with all common quantum light sources such as spontaneous parametric down-conversion sources and quantum dots. We demonstrate a variety of photonic quantum information processing primitives such as Hong-Ou-Mandel interference, bosonic coalescence and anti-coalescence and high-dimensional single-photon quantum gates, exploiting the whole mode structure of the processor. We observe that the Blass matrix processor retains the indistinguishability of the photons and enables any arbitrary linear transformation, while fully programmable and remotely controllable as desired for quantum information processing. These results show the promising future of lowloss silicon nitride waveguides for large-scale photonic quantum information processing.

Exploiting the long-term interferometric stability, low propagation loss and tight optical confinement of integrated silicon nitride waveguides, we have shown complex reconfigurable optical circuits both for classical and quantum photonic processing. For future development of integrated programmable photonic processors, various challenges need to be addressed such as compact and low-power phase shifters, a further increase of the component density and lower the propagation losses. 


\section{Samenvatting}

Programmeerbare golfgeleider-circuits zijn cruciale bouwstenen voor toepassingen in de geintegreerde spectrometrie en in de quantuminformatieverwerking. Onder de dielectrische materialen die geschikt zijn voor geintegreerde fotonica valt

siliciumnitride op wegens zijn grote bandkloof en tamelijk hoge index-contrast. Deze eigenschappen maken zowel lage propagatieverliezen in een brede spectrale band mogelijk, alsook een hoge dichtheid van componenten. Deze eigenschappen, samen met de inherente fasestabiliteit en programmeerbaarheid die mogelijk zijn in siliciumnitride, maken het mogelijk om complexe fotonische circuits te bouwen. In dit proefschrift beschrijven we fotonische circuits met een hoge integratiedichtheid en demonstreren we hun gebruik voor golflengte-metrologie en quantuminformatieverwerking.

We presenteren een nieuwe aanpak om een precieze, geintegreerde golflengte-meter te bouwen, gebaseerd op ringresonatoren en dataverwerking door middel van neurale netwerken. Een neuraal netwerk wordt getraind met een set van transmissiespectra afkomstig van een geintegreerd fotonisch circuit gebaseerd op ringresonatoren, bij verschillende instellingen van het circuit. Het circuit bestaat uit hetzij een enkele ring, hetzij een tweetal ringen in Vernier-configuratie. Vervolgens kan de transmissie van een onbekende golflengte gemeten worden met het circuit door gebruik te maken van dezelfde instellingen als bij de training. De onbekende golflengte wordt geidentificeerd door een dataverwerkingsmethode die de beste match vindt tussen de gekalibreerde spectra en het gemeten spectrum. We onderzoeken verschillende dataverwerkingsmethodes, met als doel om het golflengtebereik van het meetapparaat zo groot mogelijk te maken. We demonstreren reproduceerbaarheid over de lange termijn, alsook onafhankelijkheid van de respons van de golflengtemeter van de omgevingstemperatuur.

We presenteren ook een reconfigureerbare multibundel interferometer, die gebruikt kan worden als fotonische processor. Deze processor bevat 128 elementen in een Blass-matrix configuratie. Deze interferometer is de grootste geintegreerde fotonische processor die tot nu toe gerealiseerd is in siliciumnitride. We rapporteren over een verregaande karakterisatie van de respons van de elementen in deze processor, met name hun instellingsbereik, thermische overspraak tussen de componenten, en over de reproduceerbaarheid, respons, dispersie, en transmissie van de processor. Vanwege fabricage-imperfecties kunnen niet alle elementen ingesteld worden op elke gewenste waarde, wat de instelbaarheid van de processor beperkt. Ondanks dat 
heeft onze processor een consistente instelbaarheid over een spectraal bereik van ongeveer $150 \mathrm{~nm}$.

Na de kalibratie van de instelbare elementen bekijken we de mogelijkheid van het optimaliseren van de processorrespons, gegeven de eindige instelbaarheid van de elementen. We meten de getrouwheid van 100 unitaire transformaties die getrokken zijn uit de Haar-verdeling en onderzoeken of de gemeten getrouwheden verbeterd kunnen worden door de faseinstellingen van de elementen te optimaliseren over hun beperkte fasebereik. We demonstreren een optimalisatiealgoritme dat in staat is om tot op zekere hoogte de imperfecties op te lossen, voor een meerderheid van de gemaakte transformaties.

We demonstreren dat het eerder genoemde circuit, dat bestaat uit 8 optische kanalen, gebruikt kan worden als fotonische processor voor quantuminformatieverwerking. We demonstreren een aantal elementaire toepassingen zoals het Hong-Ou-Mandel effect, bosonische versmelting en anti-versmelting, en hoog-dimensionale enkelefoton quantumpoorten, waarbij we de hele structuur van de processor gebruiken. We zien dat de Blass matrix de ononderscheidbaarheid van de fotonen behoudt, en ons in staat stelt om een arbitraire lineaire transformatie uit te voeren, die volledig op afstand in te stellen is. Dit is een sterk gewenste eigenschap voor quantuminformatieverwerking. Deze resultaten tonen aan dat siliciumnitride een veelbelovende toekomst heeft voor grootschalige quantuminformatieverwerking. Bovendien kan siliciumnitride wegens zijn breedbandige transmissieraam gebruikt worden in combinatie met alle gebruikelijke enkelfotonbronnen, zoals parametrische down-conversie en quantum dots.

Door gebruik te maken van de interferometrische stabiliteit, lage propagatieverliezen en sterke optische begrenzing in geintegreerde siliciumnitride golfgeleiders, hebben we laten zien dat complexe, instelbare optische circuits mogelijk zijn, zowel voor klassieke golflengtemetingen als voor quantuminformatieverwerking. Voor de toekomstige ontwikkeling van geintergreerde fotonische quantumprocessoren moeten een aantal uitdagingen opgelost worden, zoals laag-verlies, compacte faseverschuivers, een verdere verhoging van de dichtheid van optische componenten, en lagere propagatieverliezen. 


\section{List of publications}

\section{Journal articles}

- $\quad$ C. Taballione, J. J. Renema, P. W. H. Pinkse, K.-J. Boller, “Characterization of a realistic photonic processor and mitigation of its fabrication imperfections". (To be submitted).

- C. Taballione, T. A. W. Wolterink, J. Lugani, A. Eckstein, B. A. Bell, R. Grootjans, I. Visscher, D. Geskus, C.G.H. Roeloffzen, J. J. Renema, I. A. Walmsley, P.W.H. Pinkse, K.-J. Boller, " $8 \times 8$ Programmable quantum photonic processor based on silicon nitride waveguides". arXiv:1805.10999 (submitted).

- C.G.H. Roeloffzen, M. Hoekman, E.J. Klein, L.S. Wevers, R.B. Timens, D. Marchenko, D. Geskus, R. Dekker, A. Alippi, R. Grootjans, A. van Rees, R. Oldenbeuving, J.P. Epping, R.G. Heideman, K. Workhoff, A. Leinse, D.H. Geuzebroek, E. Schreuder, P.W.L. van Dijk, I. Visscher, C. Taddei, Y. Fan, C. Taballione, F. Liu, D.A.I. Marpaung, L. Zhuang, M. Benelajla, K.-J. Boller, “Low loss $\mathrm{Si}_{3} \mathrm{~N}_{4}$ TriPleX optical waveguides: technology and applications overview". IEEE J. Sel. Topics Quantum Electron. 24 4, 4400321 (2018).

- M.A. Garcia Porcel, J. Mak, C. Taballione, V.K. Schermerhorn, J.P. Epping, P.J.M. van der Slot, K.-J. Boller, "Photo-induced second-order nonlinearity in stoichiometric silicon nitride waveguides". Opt. Express 25 26, 33143-33159 (2017).

\section{Conference proceedings}

- C. Taballione, T. Wolterink, J. Lugani, A. Eckstein, B. Bell, R. Grootjans, I. Visscher, D. Geskus, C. Roeloffzen, J. Renema, I. Walmsley, P. Pinkse, and K. Boller, "Si3 $\mathrm{N}_{4}$ Reconfigurable Linear Optical Network for Quantum Information Processing," in Quantum Information and Measurement (QIM) V: Quantum 
Technologies, OSA Technical Digest (Optical Society of America, 2019), paper S2C.5.

- C. Taballione, T. Wolterink, J. Lugani, A. Eckstein, B. Bell, R. Grootjans, I. Visscher, J. Renema, D. Geskus, C. Roeloffzen, I. Walmsley, P. Pinkse, and K. Boller, "8×8 Programmable Quantum Photonic Processor based on Silicon Nitride Waveguides," in Frontiers in Optics / Laser Science, OSA Technical Digest (Optical Society of America, 2018), paper JTu3A.58.

- C. Taballione, T. Agbana, G. Vdovine, M. Hoekman, L. Wevers, J. Kalkman, M. Verhaegen, P. van der Slot, and K. Boller, "Single Si3N4 Micro Ring Resonator as Integrated Wavelength Meter with Long-Term Reproducibility," in European Conference on Lasers and Electro-Optics and European Quantum Electronics Conference, (Optical Society of America, 2017), paper CK_P_27.

- $\quad$. Taballione, T. Agbana, G. Vdovine, M. Hoekman, L.S. Wevers, J. Kalkman, M.H.G. Verhaegen, P.J.M. van der Slot, K.-J. Boller, “Temperature-driftimmune wavelength meter based on an integrated micro ring resonator". Proc. of Spie 10242, 1024206 (2017). 


\section{Acknowledgements}

These last four years of PhD have been a life-changing experience and I wouldn't have made it without the support and presence of many people. It has been a long journey but we made it. Yes, we. Because this thesis, in a way or another, is also yours. Without further speaking, let's go into business.

Surviving a $\mathrm{PhD}$ is not an easy task and definitely it wouldn't have been possible without amazing people and colleagues as the ones you can find at LPN. Okki, Roel, Marteen, Lida, Xin and Bas, we have met in my highest moment of desperationby-PhD. Thanks for pretending that it was all normal. A special thank goes to "my" students Hannah, Kees, Victoria and Meryem: I have learned a lot thanks to you. Kees, you made me laugh so hard that my belly still hurts. Victoria, burning waveguides and speaking Italian with you has been a great pleasure. Meryem, your dedication to work despite the fasts was incredible. Hannah, you have been the first student to supervise in my life, plus, at the very beginning of my PhD: thanks for dealing with me so well. Let's now continue. Haider, thanks for all the talks we had, about life, marriage and science. It was very lovely to meet your wife and have dinner all together. Your genuine passion and enthusiasm for physics is overwhelming. Robert, the youngest student that LPNO have ever had! It was very funny to share opposite workstations with you. I admire your willingness to challenge yourself. You are a very pleasant person, despite your young age! Albert, you are awesome. Few words but always to the point. You are a gentleman, very kind. Thanks immensely for being the official best-ever photographer of the LPNO dance workshop and thanks for sharing your story with me. By the way...to me you are still 25 years old. It is not possible you are older. Speaking of LPNO dance workshop...Rob! The dance-addicted student of LPNO. Oh man, you made me very happy! We made the LPNO members dance!!! I mean...we made physicist dance...that's a miracle!!

Ah, the LPNO dance workshop will be one of the greatest memories of my life. Just that you know, every time I will think of it I will genuinely laugh very loud. Thanks to all the partecipants for this piece of pure happiness in my life!

Let me now mention a man of honor, the last standing $\mathrm{PhD}$ that fought side by side with me, day by day since almost the beginning: Mr. Mak. There was a time when only two soldiers were left at LPNO and those were me and you. In the loneliness of our own battles it was encouraging knowing that I had a companion. Ok, now I stop with this military metaphor. I don't even know why I am doing it... anyway! Jesse, you are a very kind and caring person. Applause for your stubbornness in turning down Klaus requests! Youwen, Yin, Kasper, Jonathan, 
Marco, Joern, Caterina and Tom, it was and absolute pleasure to share the office with you for most of my PhD. Thanks for welcoming me so warmly when I arrived. It took me two days to understand the joke of the Toblerone...but in the end I got it! (Toblerone $\sim$ Taballione) It was an absolute fun being with you guys, the talks, the jokes, lunches, PhD dinners, coffee breaks, group meetings and croissants...many memories. Yin, I am sorry for all the time I kicked you below the desk! Your jumping around and your...oh gosh I was almost forgetting it...your jokes at the end of the group meetings!!! The worse jokes on the planet...but just because you were saying them, they were totally funny! Youwen, it was a pleasure to be your paranymph and make fun of you during the presentation at your party! God knows why one day you gave me your belt ... but thanks to you, the rest is history! (I am referring to the waveguide book...nothing obscene, don't worry!) Jonathan, your humor is unique and I love it! Thanks for being so mature and honest. Marco, when I couldn't find something in the lab I knew it would have been on your table! You were always willing to help, any time, any place. It was very nice to meet you again in Valencia, thanks for the nice city tour! Joern, very synthetic. To stay on topic: you are funny. Caterina, my predecessor! I don't know what I would have done without you! You were always ready to help me. Thanks for the many laughs and for bringing a bit of Italy at LPNO. And now Tom. Ladies and Gentlemen, the most amazing guy in this world. How can I thank you enough? Simply I can't. We have shared a very big adventure in Oxford (the driving from Enschede to Oxford on an English car was awesome!) and without your help the experiments would have been simply impossible! Your dedication, kindness and sensibility are rare pearls and makes you an outstanding person. I wish you all the best of luck in finding your way.

It's time to thank now our "stage manager": Carla. Our beautiful and superefficient secretary that helps us to make our complicated life easier. It was such a blessing to have you in our group! You are fun, capable and caring. Thanks for all your help and joy! To further continue with LPNO member, I would like to mention you, David. You are a breath of fresh air bringing new life to LPNO. Thanks for the discussions we had. Your stock of knowledge is impressive. Peter, my daily supervisor (at least at the beginning). Talking with you was always very nice. Your calm and precision made your explanations a treasure of details, thanks for that. Oh, I remembered something: when I got to know that I was accepted as PhD you have been the first one to come and congratulate me...I jumped, hugged and kiss you... I think I shocked you! Sorry! Bert, Bert, Bert!! First of all, we missed your hip moves at the dance workshop, shame on you! Second of all, I really envy your holidays plan! Third, our photodetectors-array is the most beautiful baby in the world, thanks a ton for the help! And now the Boss. Klaus, is your turn! Ready for the 10 pages acknowledgement?! I am kidding...maybe...or maybe not! Seriously, probably 10 
pages would not be enough to describe how wonderful you are and how grateful I am that you gave me this opportunity. I remember distinctly our first skype call, you made me feel at ease immediately. You have been like a second father to me. The examples you gave me with your behavior are priceless and more valuable than any word. Thanks for finding the patience and strength to always, always, and again, always, explain me things. Thanks for your enormous efforts in always trying to improve me, in any way possible. Thanks for sharing my passion for music and come to support me at my shows (Peter, thank you too!!). Thanks for understanding me. Thanks for putting me under pressure. Thanks for the great opportunity you gave me. I have learned a lot in these 4 years and what I am now and will achieve in my life will be due to you.

To all LPNO members: your Queen blesses you. But treat my waveguides right otherwise I am gonna use Youwen's belt!!

Spending 4 years at the University means a lot of lunch breaks. One constant presence to these lunch breaks were the COPS members: Manashee, Shweta, Tristan, Jan, Rebecca, Chris, Matthijs, Maryna, Diana, Ravi, Reinier, Evangelos, Jelmer, Pepijn, Cock, Nicole and Willem. It was very nice to share that time with all of you guys...but remember, we will always be at the other side of the table! Reinier, we could work a bit together and I totally enjoyed it! Willem, many thanks to you too! That was one of the epic moments of my life, to see you in action! Jelmer, the quantum Viking, as Klaus would call you. I am happy you came to visit Twente looking for waveguides, because there all started. I am even happier that you moved to Twente from Oxford, working with you for my thesis was always nice, interesting and useful. Thanks for the discussions, explanations and tips you gave me. It is a total pleasure to keep working with you at QuiX. The dinner at the high table at your college in Oxford, that was fun! Thanks for the experience! Oh, and now that you just sent it, thanks for translating the summary in dutch! A very special thank now goes to Pepijn. I have known you since the beginning but only at the end of my PhD you became my supervisor. And I am so happy that this happened! You have been a great support and help. Beside the science, I totally enjoyed singing with you in the Christmas choir of the Mesa+. Maybe we will have the chance to sing together again! Another special thank goes to Evangelos for being always there when I needed the most. Thanks for listening so much and for being such a fan of me. Your support has been vital to me. You are doing something great for your life and I wish you all the success that you deserve!

I would like to thank the OS group for sharing and enjoying with us all the retreats and parties. You are a lovely bunch of people, so thank you Herman, Sonia, Jennifer, Jose, Jeroen, Frans, Meindert, Lantian, Sandra, Carlijn, Raimond, Michiel, Ward, Wooje, Simen and Jinfeng. Michiel, you know I love you but karaoke is not 
your thing, man... kidding, keep doing it! I really enjoyed it! Ward and Simen thanks for the coffee workshop, that was fun and interesting! Jinfeng, discussing with you about ring resonators and silicon nitride was always very nice! Congratulations again for your recent graduation!

A lot of the work in this thesis wouldn't have been possible without valuable people as Ruud, Robert, Ilka, Lennart, Marcel, Edwin, Chris, Rene and Hans from LioniX. Thanks for being always willing to discuss and confront with me about how to make what I had in my head real.

As I already mentioned, I have experienced a great adventure in Oxford and also there I met amazing people that made me feel less lost. Let me thank first Prof. Ian Walmsley for being a silent but essential enabler of the collaboration between Twente and Oxford. Then I would like to thank Jasleen, Andreas and Bryn for contributing to the experiments. Jasleen you have been a great support throughout my whole stay, thank you very much. Joelle!! Meeting you back in Oxford after our Master in Rome was super nice! I fully enjoyed our talks and dinners together!! A lot of other people in Oxford deserve a thank: Thomas Hird (it was nice to meet you all around the globe!), Alex, Patrick, David and William for the nice talks and company!

Beside the academic world, I have to thank a lot of people that helped me getting distracted from the $\mathrm{PhD}$ and remember me that there are many ways of enjoying life. As you might know I love music, dancing and especially singing. Moving to Enschede has been a blessing for me, almost like a spiritual retreat. In this place of calm and peace (sometimes way too much!) I have found back my voice, the mirror of my soul. Here in Enschede I fortunately had the chance to enjoy my passions in many different ways.

A warm thank goes to Janneke, Manashee, Pepijn, Jaqueline, Dodo, Telma, Jan, Marko, David and Egbert for making the Christmas choir of Mesa+ so much fun!

The Ex Arte choir has also been a place of joy in these past crazy months, I am only sorry that I could not participate more. Thanks to all the members, especially Emil, Carine, Marie, Arend and Meike!

I really would like to thank an amazing person that happened to be my singing teacher for a bit, Annelies Lamm. Thanks for your patience and your enormous support! I enjoyed every second of our classes.

The crew of West Side Story, cast \& decor, gets all my love. It was an amazing adventure, very hard work, but you guys made it totally easy to do. I have found amazing people to share my passion with. We spent so many hours rehearsing and still we enjoyed every damn second. Thanks to each of you for everything you did on and back stage. Vivian, acting and singing with you was just perfect. Mitchell, it's a pity that we didn't have many scenes together but I enjoyed a lot saying to you Buenas No..oooooo...ches! Kasper, 'Nardo, my crazy love, Te quiero! To the Sharks: 
you have been a great support to my Nardo...keep fighting!! To the Jets: Victor, Ryan, Brad, Jan, Jonah, Adi and Marit. You guys were amazing, an absolute thrill to watch you. I enjoyed very much working with you all. Thanks for being so gentle with Anita in Act II. To my Shark girls Meike, Alina, Tanja, Luise, Femke and ok, also the Jets girls Brenda, Minke, Allegra, Romy, Sophie: I love you girls. Thanks for making the show so awesome and America the coolest piece of the musical! Anouk, Doc, you were absolutely amazing! Henk, the transformer of the show, kudos to your performance! Manu, your capability of intimidating was great! Jim, my shy Chino. I always believed you, and I was always afraid that you were going really to break Vivian's arm! Simon, amazing Pepe. Your break dance made us the coolest! A last thank to Melanie, Robert and Peter the mystic triad keeping up the show. It was fantastic to work with you! To every one of the Décor team, Carlijn, Willem, Laurens, Peter and Wouter and the rest...without you we would have been just a bunch of crazy people jumping on a stage. You made the dream come true!!

Another important person of my life that I would like to thank if Josef. I have met you via Evangelos (for which I will always thank him!) during my first audition and in the end I found a good friend. I love you very much and I love your pure, honest and vibrating soul.

Together with my singing passion, in this last year I developed a real passion to dancing salsa. Or to be more specific, I became addicted to Rico Latino! There I have found awesome people and friends: Nel, Liza, Lindsay, Ozlem, Vera, Sandy, Michael, Angela, Roberto, Daphne, Zjurieck, Pritam and Pallav. Thank you for all the dances, laughs and jokes we shared. I hope my chicken-, crab-, Egyptian- and Italian-dance will remain in the history of Rico...I am sure they will! You all made me feel like home and I will always be grateful to all of you for this. Thanks to Rico (I should say Michael!) I have found you, Carmela. God knows how we did not manage to talk to each other for 1 year being in the same place. But fortunately it happened and we just clicked. You are a strong, determined, passionate, beautiful and caring woman. You have no idea how important has been your presence, help, support in this last period of my life. Our vocal messages on Whatsapp have reached embarassing lengths but... who cares!

Now I would like to thank my Italian crew that has been coming and going through the 4 years of my PhD: 1'Ila e il Save and Lucia e Antonio. Guys, fortunately me and Giorgio found you. Enschede would have been a much worse place without you! You gave me moment of pure Italianism that warmed up my heart... and made me laugh very loud! Ilaria, the only person to define Enschede as a "gioiellino" and run away from it as fast as she could! I love you, you have been an immense support to me. 
Last but not least, my family. And here I will switch to Italian, sorry guys!

Alla mia famiglia, che adoro dal più profondo del mio cuore. Senza di voi non sarei la persona che sono ora. Mi siete mancati come l'aria, ogni giorno. Grazie per gioire sempre con me dei miei successi e consolarmi nei momenti difficili. Grazie anche per rompere le scatole, perché ogni tanto, diciamolo, ci vuole. Teresa e Adriano, mamma e papà, siete i miei pilastri [1][2]. Il vostro sostegno è sempre stato fondamentale e il vostro esempio è l'insegnamento più prezioso che potessi ricevere. Alessandro e Paolo, i miei fratelli maggiori. Siete il motore che mi spinge a fare sempre di più e a fare sempre meglio. Perché mamma si vanta talmente tanto di voi che pure io mi devo far notare! Alle mie cognate bellissime Manuela e Julie, che sopportano i miei fratelli. Siete la dolcezza e l'amore di questa famiglia, grazie per essere le mie alleate! Ai miei nipoti Alma, Anita, Amanda e Matteo. Le mie stelle e i miei soli. Mi avete regalato i sorrisi più grandi e le risate più sonore di questi quattro anni.

Alla mia famiglia acquisita: Elisabetta, Riccardo, Anna, Marco e Valentina. Mille volte grazie per avermi accolto letteralmente come una figlia nella vostra famiglia. Non potevo essere più fortunata. Ogni volta che vi vedo è una festa. Elisabetta sei la nonna che mi è sempre mancata vicino. Riccardo, il suocero più pazzo del mondo. Giorgio è la tua copia esatta...quantomeno so cosa mi aspetta!! Grazie infinite per le macchine...sarei morta di broncopolmonite al primo inverno qui in Olanda senza! E senza di te quel giorno, non sarei mai andata in ospedale, quindi grazie di cuore. Anna, solo tu mi puoi capire!!! Il tuo amore e sostegno fanno bene al cuore! E grazie per l'infinità di crostate che ci hai preparato, quelle fanno bene allo stomaco! Marco, praticamente mio fratello. Grazie per avermi tenuto vicino quando Giorgio era lontano. Valentina, che senza vergogna ha provato a rifilare a mia madre un'assicurazione sulla casa durante una cena. Sei fantastica, super brillante e io ti adoro!

Dulcis in fundo, mio marito. Croce e delizia del mio cuore. Giorgio, unico nel suo genere. Vivere senza di te è stata una delle cose più difficili che io abbia mai dovuto affrontare nella mia vita. Sei la mia anima gemella, un compagno sincero e onesto. Sei la mia casa e il mio rifugio. Grazie per capire quali sono le cose importanti per me e per sostenermi, sempre e comunque. Grazie.

[1] Mariani T., "Aqquande Aqquande," dal libro E' tutta questione di organizzazione (1952).

[2] Taballione A., “Ammesso e non concesso" dal best seller Non è che! (1949). 